\title{
PHASE I REPORT: DARPA EXOSKELETON PROGRAM
}

\section{January 2004}

Assembled and edited by B. S. Richardson

Other contributors

J. F. Jansen *

J. F. Birdwell*

A. C. Boynton ${ }^{\dagger}$

H. P. Crowell, III

W. K. Durfee ${ }^{\ddagger}$

J. D. Gongola

S. M. Killough ${ }^{\star}$

D. J. Leo

R. F. Lind*

L. J. Love*

M. Mungiole ${ }^{\dagger}$

F. G. Pin

J. C. Rowe*

O. A. Velev

T. Zambrano $\$$

* Oak Ridge National Laboratory

${ }^{\dagger}$ Army Research Laboratory

₹ University of Minnesota

$\S$ AeroVironment, Inc.

* Virginia Polytechnic Institute 


\title{
DOCUMENT AVAILABILITY
}

Reports produced after January 1, 1996, are generally available free via the U.S. Department of Energy (DOE) Information Bridge:

Web site: http://www.osti.gov/bridge

Reports produced before January 1, 1996, may be purchased by members of the public from the following source:

\author{
National Technical Information Service \\ 5285 Port Royal Road \\ Springfield, VA 22161 \\ Telephone: 703-605-6000 (1-800-553-6847) \\ TDD: $703-487-4639$ \\ Fax: 703-605-6900 \\ E-mail: info@ntis.fedworld.gov \\ Web site: http://www.ntis.gov/support/ordernowabout.htm
}

Reports are available to DOE employees, DOE contractors, Energy Technology Data Exchange (ETDE) representatives, and International Nuclear Information System (INIS) representatives from the following source:

Office of Scientific and Technical Information

P.O. Box 62

Oak Ridge, TN 37831

Telephone: 865-576-8401

Fax: 865-576-5728

E-mail: reports@adonis.osti.gov

Web site: http://www.osti.gov/contact.html

This report was prepared as an account of work sponsored by an agency of the United States Government. Neither the United States government nor any agency thereof, nor any of their employees, makes any warranty, express or implied, or assumes any legal liability or responsibility for the accuracy, completeness, or usefulness of any information, apparatus, product, or process disclosed, or represents that its use would not infringe privately owned rights. Reference herein to any specific commercial product, process, or service by trade name, trademark, manufacturer, or otherwise, does not necessarily constitute or imply its endorsement, recommendation, or favoring by the United States Government or any agency thereof. The views and opinions of authors expressed herein do not necessarily state or reflect those of the United States Government or any agency thereof. 


\title{
PHASE I REPORT: DARPA EXOSKELETON PROGRAM
}

\author{
Assembled and edited by B. S. Richardson \\ Other contributors \\ J. F. Jansen \\ J. F. Birdwell \\ A. C. Boynton \\ H. P. Crowell, III \\ W. K. Durfee \\ J. D. Gongola \\ S. M. Killough \\ D. J. Leo \\ R. F. Lind \\ L. J. Love \\ M. Mungiole \\ F. G. Pin \\ J. C. Rowe \\ O. A. Velev \\ T. Zambrano
}

Date Published: January 2004

Prepared by the Oak Ridge National Laboratory

Oak Ridge, Tennessee 37831

Managed by

UT-Battelle, LLC

for the

U.S. Department of Energy

Under contract DE-AC05-OR22725 



\section{TABLE OF CONTENTS}

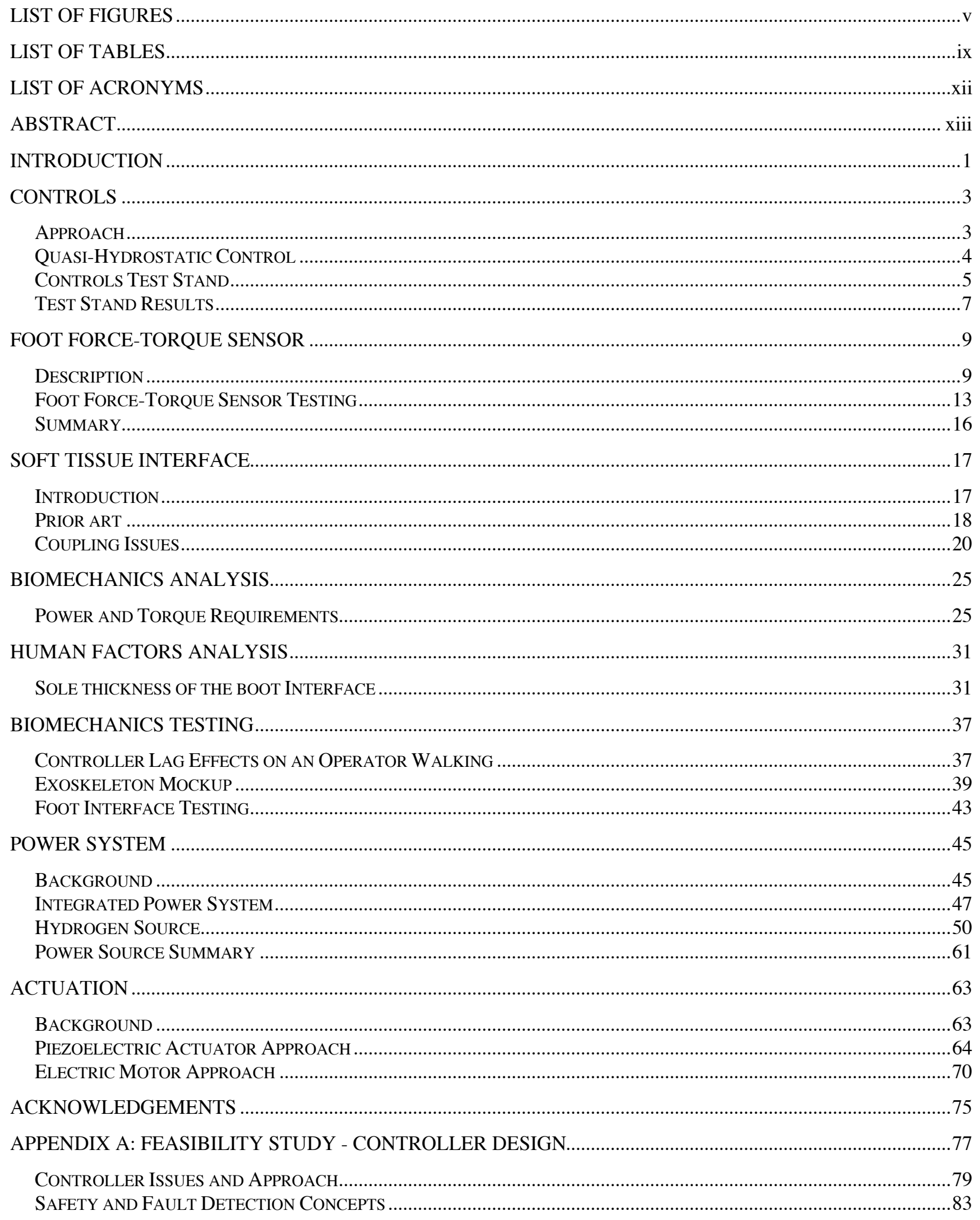




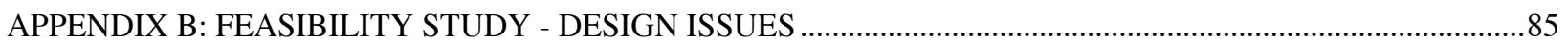

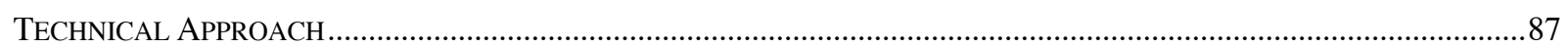

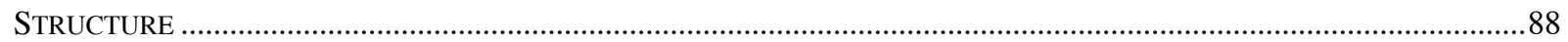

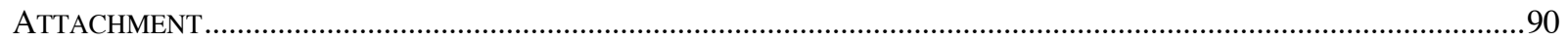

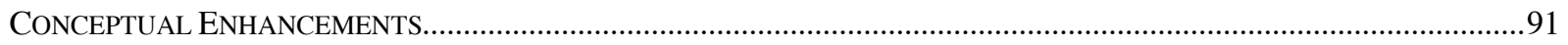

APPENDIX C: POWER AND TORQUE DATA ……………..........................................................................93

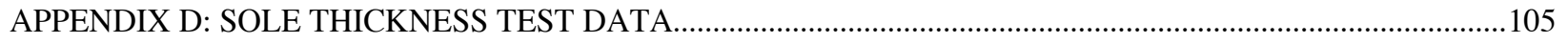

APPENDIX E: FEASIBILITY STUDY - POWER SOURCE COMPARISON N.............................................................113

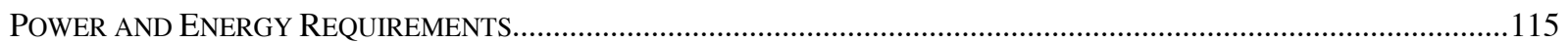

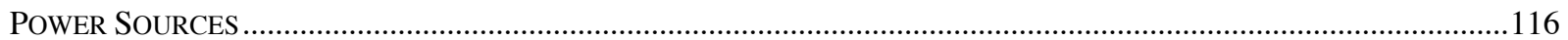

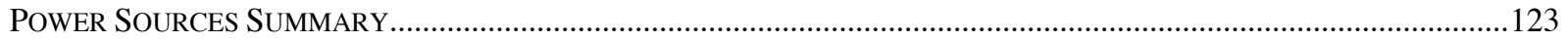

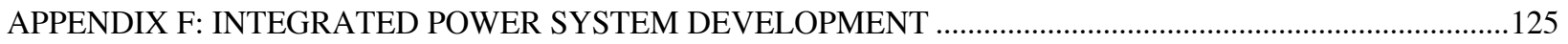

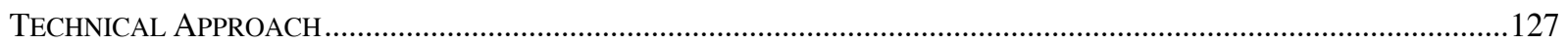

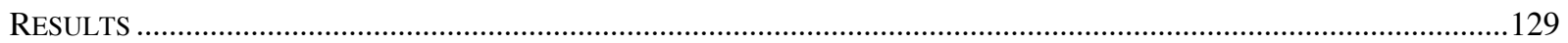

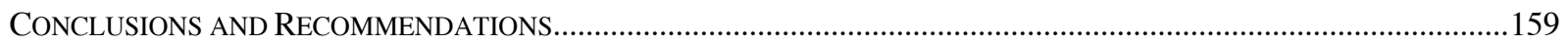

APPENDIX G: FEASIBILITY STUDY - ACTUATOR COMPARISON ……………………………………........161

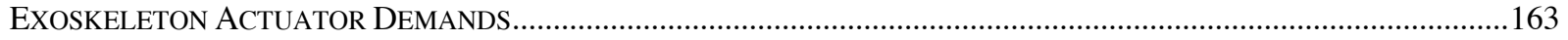

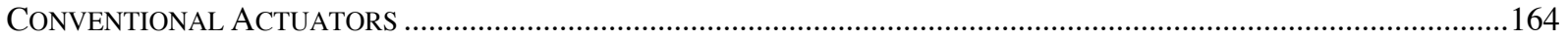

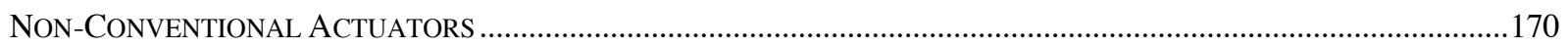

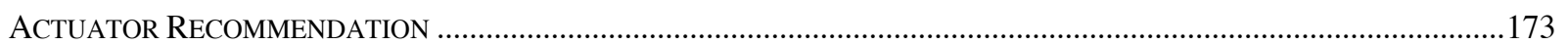

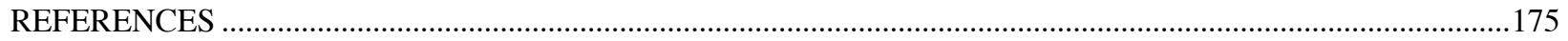




\section{LIST OF FIGURES}

Fig. 1. Leg structure and sensing concept. ............................................................ 4

Fig. 2. Power requirement, constant pressure ....................................................... 5

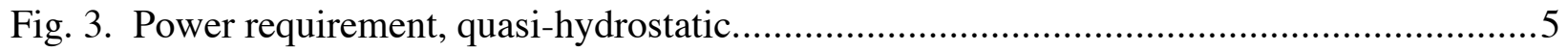

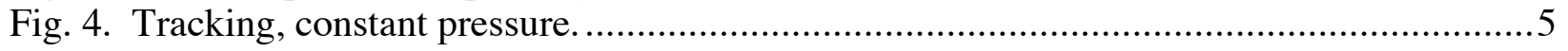

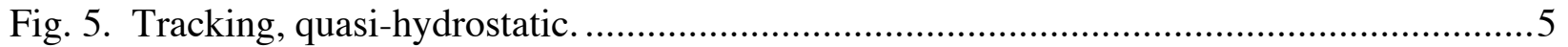

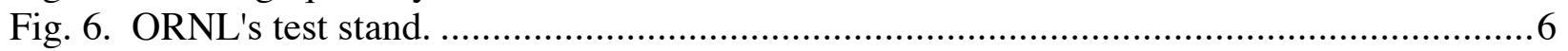

Fig. 7. ORNL's test stand physical layout................................................................ 6

Fig. 8. ORNL's test stand sensor layout. ............................................................. 7

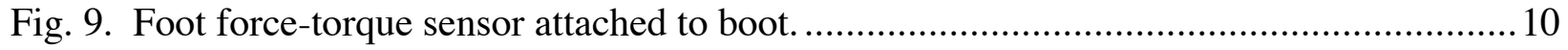

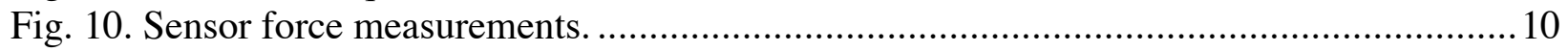

Fig. 11. Prototype foot sensor load cell details (bottom view) ............................................ 11

Fig. 12. Force-torque sensor shown flat and flexing. ............................................... 11

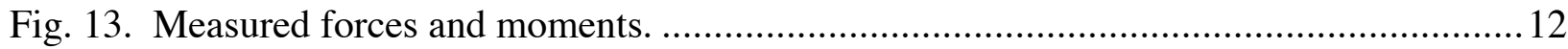

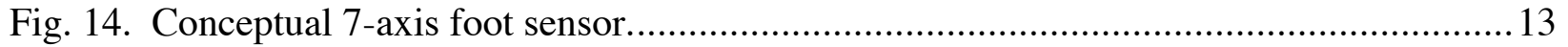

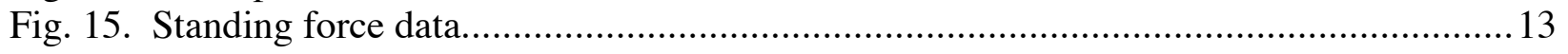

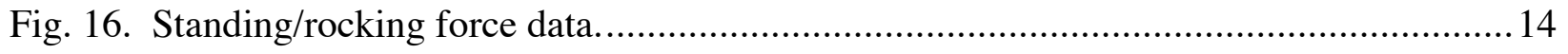

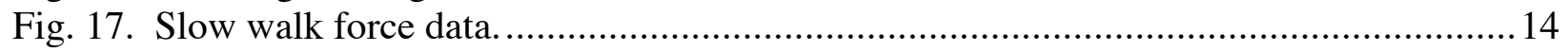

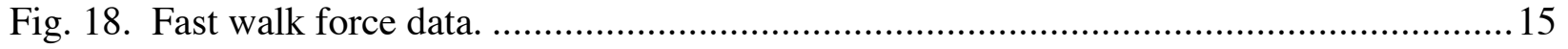

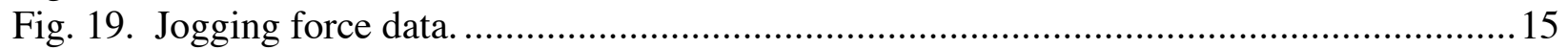

Fig. 20. Jumping force data. .................................................................................... 16

Fig. 21. Layers at the foot coupling. ...................................................................... 21

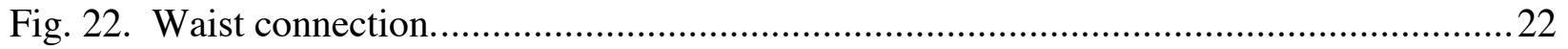

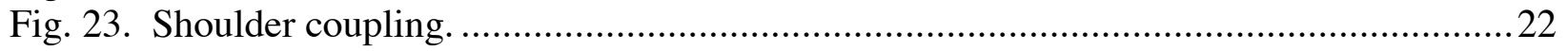

Fig. 24. Common orthotics industry materials............................................................ 23

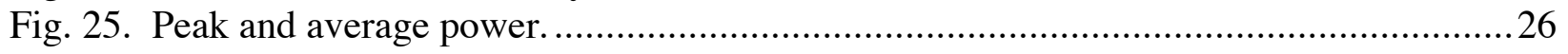

Fig. 26. Lower limb peak power profile for clear-building mission scenario..........................28

Fig. 27. Lower limb peak power profile for movement-to-contact mission scenario. ................28

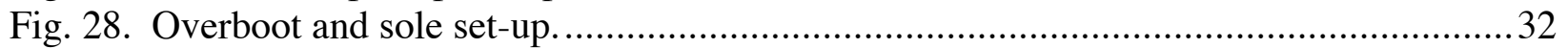

Fig. 29. Reflective marker placement. ...................................................................... 32

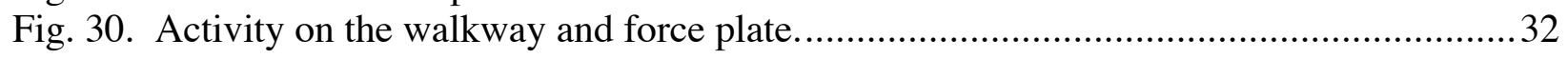

Fig. 31. Obstacles on the ARL mobility-portability course ......................................... 33

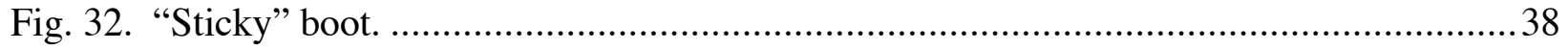

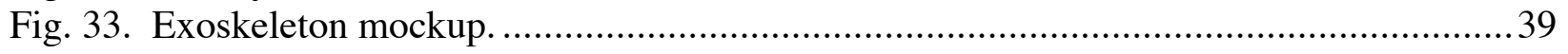

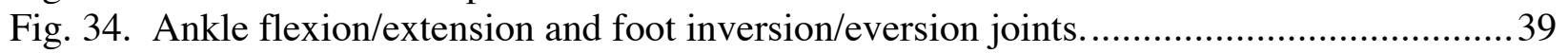

Fig. 35. Knee flexion/extension and thigh prismatic joints............................................... 40

Fig. 36. Hip flexion/extension and internal/external rotation joints................................ 40

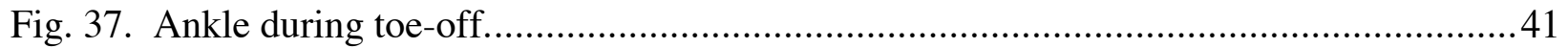

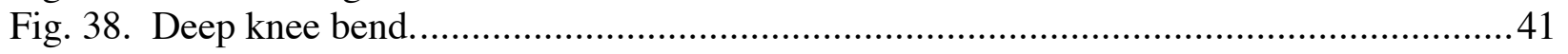

Fig. 39. Foot inversion. .......................................................................................... 42

Fig. 40. Ankle flexion/extension motions............................................................... 42

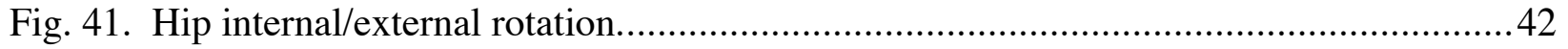

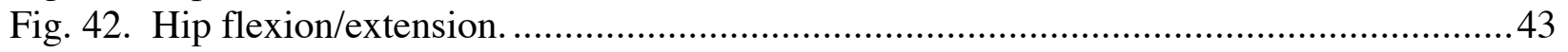

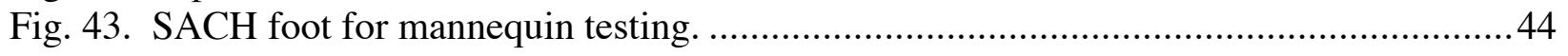


Fig. 44. Testing shoe/foot interface loads. ................................................................... 44

Fig. 45. Comparison of different power sources. ........................................................... 45

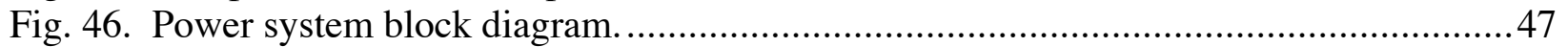

Fig. 47. Integrated power test bed........................................................................ 48

Fig. 48. Power testbed experimental data. ...................................................................... 48

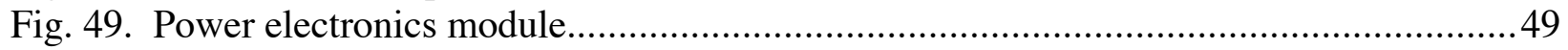

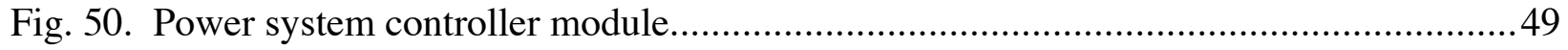

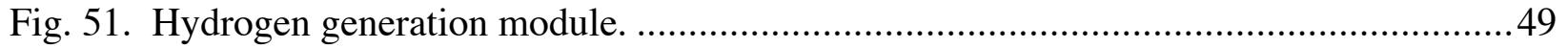

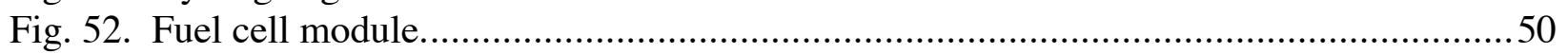

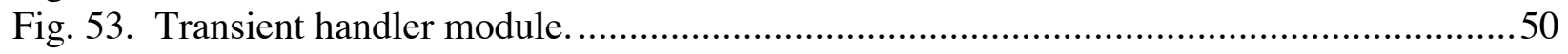

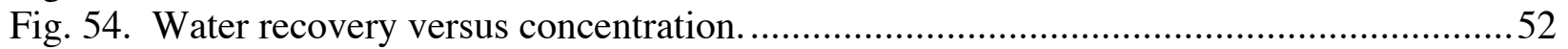

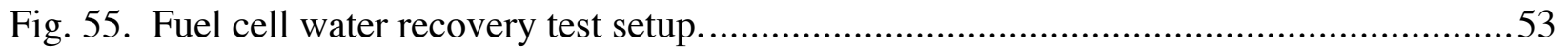

Fig. 56. Solubility of boric acid and several sodium borates. ..........................................54

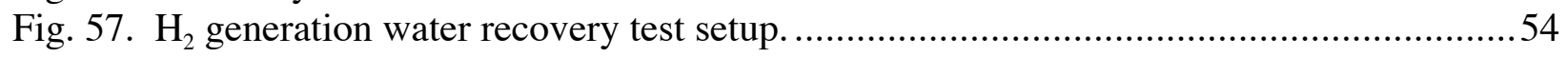

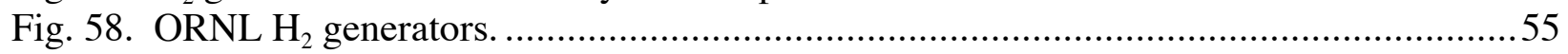

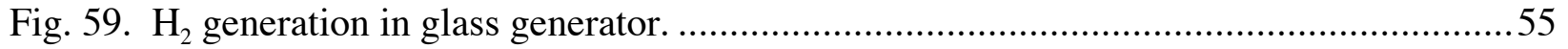

Fig. 60. Test data and water recovery versus concentration. ..........................................56

Fig. 61. Solubility of $\mathrm{NaBH}_{4} \mathrm{vs}$ temperature .............................................................5

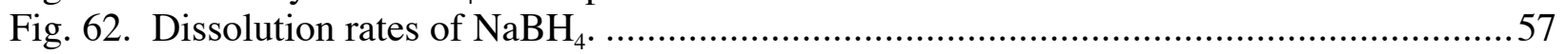

Fig. 63. Experiment configuration..........................................................................5

Fig. 64. Hydrogen generation reaction rates using ruthenium catalyst..................................59

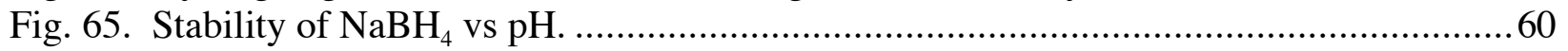

Fig. 66. Stability of NaBH4 solution vs time at different temperatures. ..............................6 60

Fig. 67. Hydrogen off-gassing from $\mathrm{NaBH}_{4}$ solution. ...................................................61

Fig. 68. Hydrogen generation and fuel cell system block diagram (with water recycling)........62

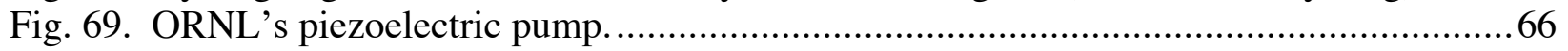

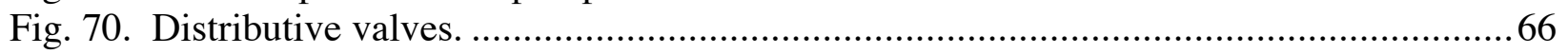

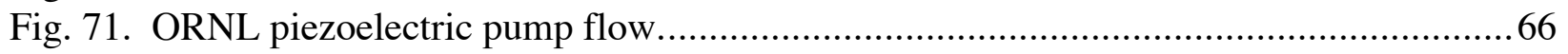

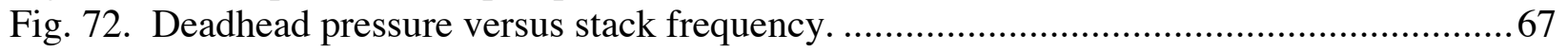

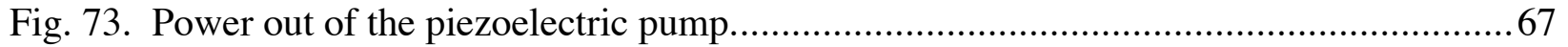

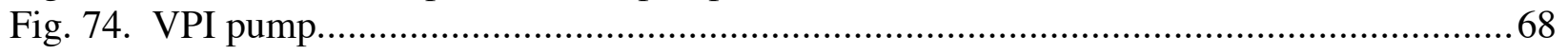

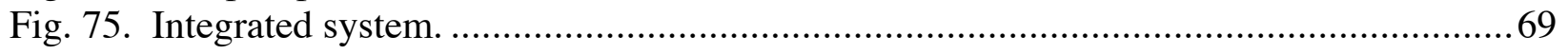

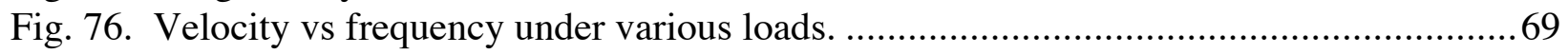

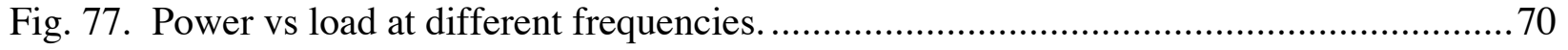

Fig. 78. Flow requirement of pump. .............................................................................. 72

Fig. 79. Pressure requirement of pump......................................................................... 72

Fig. 80. Power requirement of pump. ........................................................................ 73

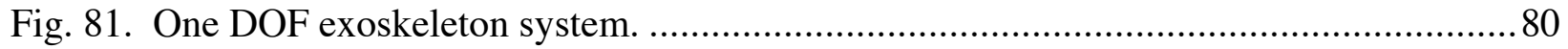

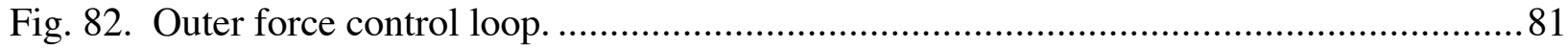

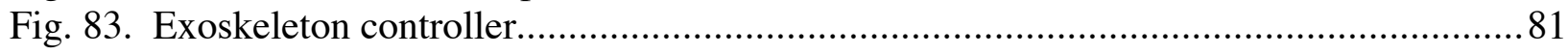

Fig. 84. NGMH fault detection (normal operation and sensor failure). ............................. 84

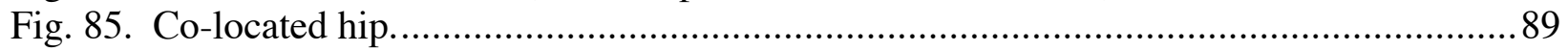

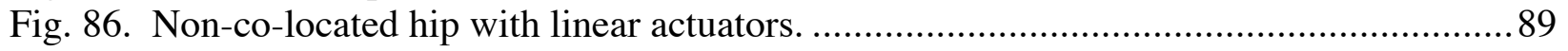

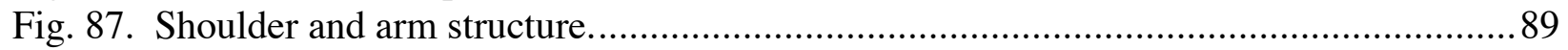

Fig. 88. Foot attachment schematic. ...................................................................... 91

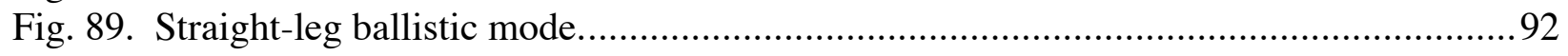


Fig. 90. Leg structure schematic demonstrating thigh prismatic joint.................................92

Fig. 91. Hip joint angles, moments, and powers - walking............................................. 95

Fig. 92. Knee joint angles, moments and powers - walking. ............................................ 96

Fig. 93. Ankle joint angles, moments and powers - walking............................................ 97

Fig. 94. Joint angles and powers - walking. ................................................................ 98

Fig. 95. Joint angles and powers - walking with various loads. .......................................99

Fig. 96. Joint moments and powers - moderate running. ............................................... 100

Fig. 97. Joint moments and powers - running at varying velocity. .................................. 101

Fig. 98. Joint moments - stair ascent and descent....................................................... 102

Fig. 99. Sagittal plane joint powers - one cycle of stair ascent and descent. ........................ 103

Fig. 100. Joint moments and powers - running vertical jump. .......................................... 104

Fig. 101. Mean comfort, stability and difficulty questionaire responses............................ 112

Fig. 102. Comparison of different power sources. ...................................................... 116

Fig. 103. Battery Ragone Diagram........................................................................ 122

Fig. 104. Super capacitor energy storage vs mass and volume...................................... 123

Fig. 105. Super capacitor peak power vs mass and volume........................................... 123

Fig. 106. Specific power curves for Panasonic HHR200SCP batteries................................. 133

Fig. 107. Specific power curves for Panasonic HHR300SCP batteries................................. 133

Fig. 108. Specific power curves for Moltech EMH-1550C batteries. .................................... 134

Fig. 109. Specific power curves for Moltech EMH-2200C batteries. .................................... 134

Fig. 110. Polarization curve for $\mathrm{E}_{0}=0.95, \mathrm{~b}=0.06 \mathrm{~V} / \mathrm{dec}, \mathrm{R}=0.26 \square . \mathrm{cm}^{2}, \mathrm{p}=\mathrm{p}_{0} \ldots \ldots \ldots \ldots \ldots \ldots \ldots . .138$

Fig. 111. Steady state model of the fuel cell stack. ....................................................... 140

Fig. 112. Schematic diagram of the experimental set-up.......................................... 142

Fig. 113. Typical measured dependence of stack voltage............................................. 143

Fig. 114. Stack voltage and current as a function of time. Pulse width 20 s........................ 144

Fig. 115. Stack power, voltage and current as a function of time. Pulse width $20 \mathrm{~s}$.............. 144

Fig. 116. Stack power, voltage and current as a function of time. Pulse width $20 \mathrm{~s}$.............. 145

Fig. 117. Stack voltage and current as a function of time. Pulse width 180 s...................... 145

Fig. 118. Stack power, voltage and current as a function of time. Pulse width 180 s .............. 146

Fig. 119. Energy storage system control diagram. ......................................................... 147

Fig. 120. Hybrid fuel cell system diagram................................................................... 148

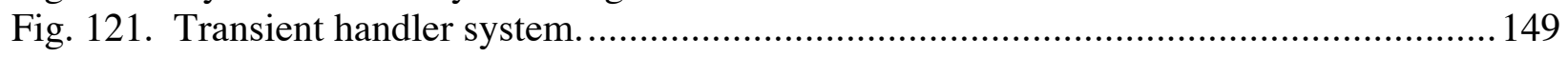

Fig. 122. Pulse load system................................................................................... 150

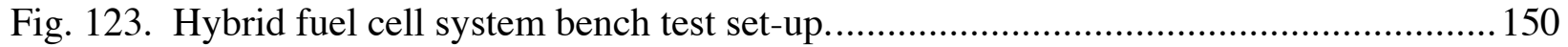

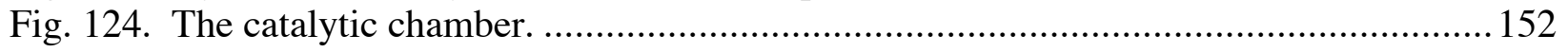

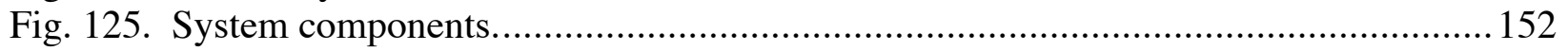

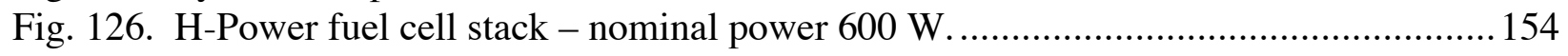

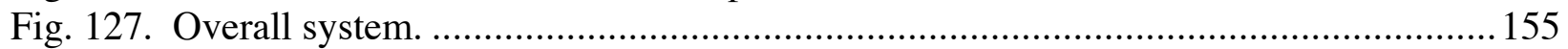

Fig. 128. Experimental data of fuel cell and transient handler............................................ 155

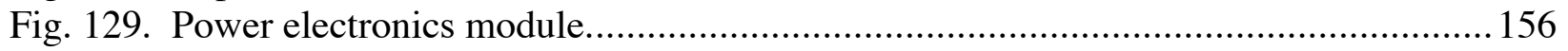

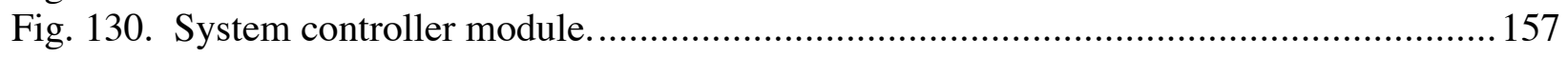

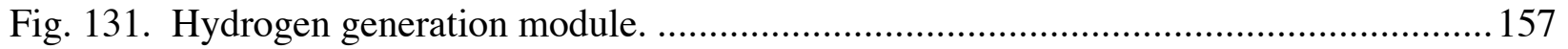

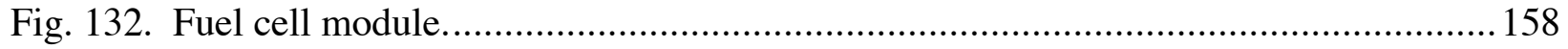

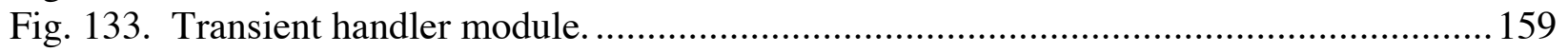

Fig. 134. Power vs mass for frameless brushless de motors. ........................................... 164

Fig. 135. Torque per unit motor volume vs continuous power. ....................................... 165 
Fig. 136. Torque per mass vs power continuous. .................................................... 165

Fig. 137. Speed (revolutions/s) vs continuous power............................................ 167

Fig. 138. Electromechanical model of piezoelectric actuator with mechanical load. .............. 171

Fig. 139. Magnetostrictive electromechanical model................................................. 172 


\section{LIST OF TABLES}

Table 1. Sample time-line of a typical soldier mission.......................................................2 27

Table 2. Peak and average power values for typical soldier tasks ........................................27

Table 3. Power source comparison ........................................................................... 46

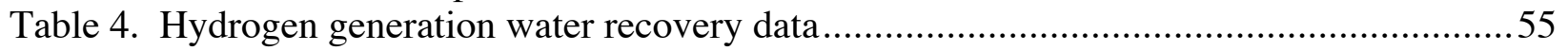

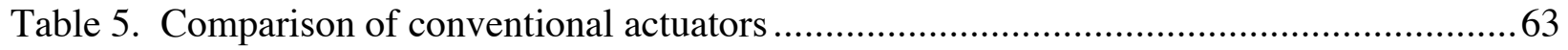

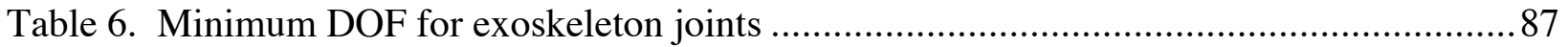

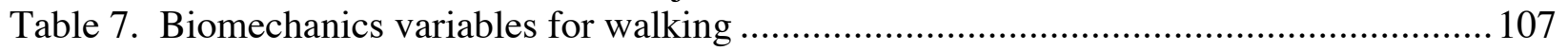

Table 8. Biomechanics variables for running .................................................................. 108

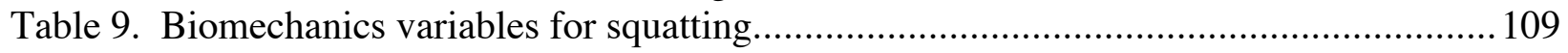

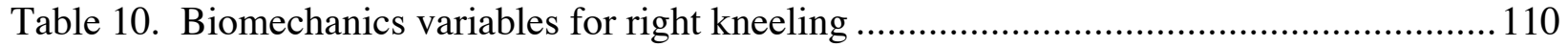

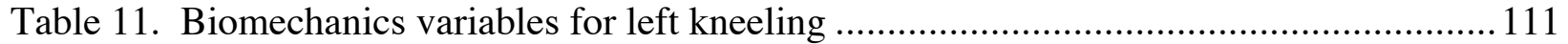

Table 12. Obstacle completion times.......................................................................... 112

Table 13. Comparison of fuel cell types ............................................................... 117

Table 14. Comparison of hydrogen-generating chemical reactions ................................... 119

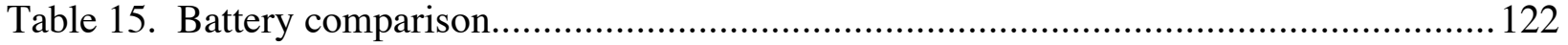

Table 16. Power source comparison ............................................................................... 124

Table 17. Peak specific power of various commercial NiMH Cells ..................................... 130

Table 18. Pack voltage for $1.5 \mathrm{~kW}$ discharge capabilities .................................................. 135

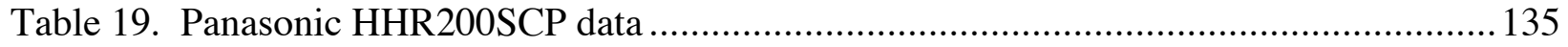

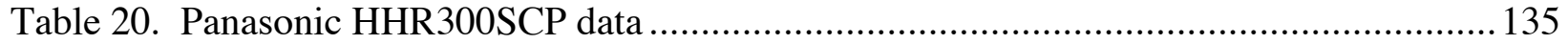

Table 21. Moltech EMH-1550C data............................................................................ 136

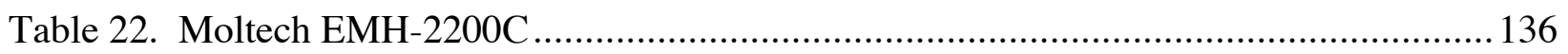

Table 23. Calculated electrochemical fuel cell parameters........................................... 141

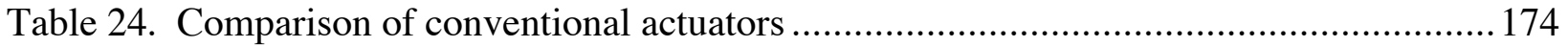




\section{LIST OF ACRONYMS}

ANOVA - Analysis of Variance

ARL - Army Research Laboratory

BSC - Battery System Controller

CAD - Computer Aided Design

DARPA - Defense Advanced Research Projects Agency

DOF - Degree-of-Freedom

EAP - Electroactive Polymer

EHPA - Exoskeleton for Human Performance Augmentation

HGO - Hip Guidance Orthosis

HKAFO - Hip-Knee-Ankle-Foot Orthosis

ICE - Internal Combustion Engine

LSD - Least Significant Difference

NSK - Nippon Seiko

NiMH - Nickel-Metal-Hydride

ORNL - Oak Ridge National Laboratory

PZN - Lead Zirconate Niobate

PZN-PT - Lead Zirconate Niobate / Lead Titanate

PZT - Lead Zirconate Titanate

RGO - Reciprocating Gait Orthosis

SACH - Solid-Ankle, Cushioned Heel

SMA - Shaped Memory Alloy

VPI - Virginia Polytechnic Institute 


\section{ABSTRACT}

The Defense Advanced Research Projects Agency (DARPA) inaugurated a program addressing research and development for an Exoskeleton for Human Performance Augmentation in FY 2001. A team consisting of Oak Ridge National Laboratory, the prime contractor, AeroVironment, Inc., the Army Research Laboratory, the University of Minnesota, and the Virginia Polytechnic Institute has recently completed an 18-month Phase I effort in support of this DARPA program. The Phase I effort focused on the development and proof-of-concept demonstrations for key enabling technologies, laying the foundation for subsequently building and demonstrating a prototype exoskeleton.

The overall approach was driven by the need to optimize energy efficiency while providing a system that augmented the operator in as transparent manner as possible (non-impeding). These needs led to the evolution of two key distinguishing features of this team's approach. The first is the "no knee contact" concept. This concept is dependent on a unique Cartesian-based control scheme that uses force sensing at the foot and backpack attachments to allow the exoskeleton to closely follow the operator while avoiding the difficulty of connecting and sensing position at the knee. The second is an emphasis on energy efficiency manifested by an energetic, power, actuation and controls approach designed to enhance energy efficiency as well as a reconfigurable kinematic structure that provides a non-anthropomorphic configuration to support an energy saving long-range march/transport mode.

The enabling technologies addressed in the first phase were controls and sensing, the soft tissue interface between the machine and the operator, the power system, and actuation. The controller approach was implemented and demonstrated on a test stand with an actual operator. Control stability, low operator fatigue, force amplification and the human interface were all successfully demonstrated, validating the controls approach. A unique, lightweight, low profile, multi-axis foot sensor (an integral element of the controls approach) was designed, fabricated, and its performance verified. A preliminary conceptual design of the human coupling and soft tissue interface, based on biomechanics research has been developed along with a test plan to support an iterative design process. The power system concept, a fuel cell hybrid power supply using chemical generated hydrogen, was successfully demonstrated and shown to be able to efficiently meet both steady-state and transient peak loads. Two actuator approaches, a piezoelectric actuator, with theoretical high power densities and an approach based on a high-performance, high-speed electric motor driving a miniature hydraulic pump have been investigated. The first shows great potential but will require further research before reaching that promise. The other approach has been modeled and simulated and shown to provide the possibility for significant energy savings (>30\%) and improved power densities in comparison to conventional hydraulics.

Biomechanics analysis and testing were also performed in support of these enabling technologies, to provide a basis for design criteria. An analysis was performed to determine baseline data for initial mechanical design and power supply sizing. Testing conducted to evaluate boot sole thickness found that thickness increases up to two inches could be accommodated without significant impact on human factors issues.

This 18-month long Phase I effort has evaluated key enabling technologies and demonstrated advances in these technologies that have significantly increased the likelihood of building a functional prototype exoskeleton. 


\section{INTRODUCTION}

The Defense Advanced Research Projects Agency (DARPA) inaugurated a program addressing research and development for an Exoskeleton for Human Performance Augmentation in FY 2001. A team consisting of Oak Ridge National Laboratory, the prime contractor, AeroVironment, Inc., the Army Research Laboratory, the University of Minnesota, and the Virginia Polytechnic Institute has recently completed an 18-month Phase I effort in support of this DARPA program. The Phase I effort focused on the development and proof-of-concept demonstrations for key enabling technologies, laying the foundation for subsequently building and demonstrating a prototype exoskeleton.

The overall approach was driven by the need to optimize energy efficiency while providing a system that augmented the operator in as transparent manner as possible (non-impeding). These needs led to the evolution of two key distinguishing features of this teams approach. The first is the "no knee contact" concept. This concept is dependent on a unique Cartesian-based control scheme that uses force sensing at the foot and backpack attachments to allow the exoskeleton to closely follow the operator while avoiding the difficulty of connecting and sensing position at the knee. The second is an emphasis on energy efficiency manifested by an energetic, power, actuation and controls approach designed to enhance energy efficiency as well as a reconfigurable kinematic structure that provides a non-anthropomorphic configuration to support an energy saving long-range march/transport mode.

The enabling technologies addressed in the first phase were controls and sensing, the soft tissue interface between the machine and the operator, the power system, and actuation. Biomechanics analysis and testing were also performed in support of these enabling technologies, to provide a basis for design criteria. This document describes the advances made in the 18-month long Phase I effort for each of these key enabling technologies and how these advances have increased the likelihood of building a functional prototype exoskeleton. 


\section{CONTROLS}

\section{APPROACH}

The Oak Ridge National Laboratory's (ORNL's) controls approach is based on three requirements: (1) the human operator will perform all the balancing, (2) small amount of force due to the load will be reflected back to the human, and (3) only two physical contact points per leg with the human are allowed. These three requirements necessitate a bilateral force-based controller. While the final exoskeleton system would be composed of arm, torso, and leg structures, the leg structure is the major emphasis of the first phase of the exoskeleton program due to its difficulty and uniqueness and will be the only exoskeleton structure discussed in detail.

While biped control for autonomous robots is still an active research area, its application to an exoskeleton is almost negligible because the balancing capability of the human operator will be utilized by the exoskeleton. The transfer of balancing capability from the operator to the exoskeleton is based on bilateral force reflection. Historically, the major application of bilateral force reflection controls has been for teleoperator (master-slave) systems. There are three general types of control strategies used in a teleoperator system: (1) position-position, (2) position-force, and (3) force-force control. The first control strategy, which was used on the Hardiman, refers to the type of sensory information (in this case positional information) being used in the "outer" feedback loop of both the master and slave. Position-position control is commonly implemented in most teleoperator systems. Two teleoperators built at ORNL in the 1980s, the M2 and the Advanced Servo Manipulators, are examples of this type of control strategy that is common for systems that have kinematically similar master-slave manipulators. For kinematically dissimilar master-slave systems, position-force control is commonly used. For human-amplifier (or extender) type systems, where the master and slave are one integral unit such that the human operator and the manipulator are attached to a common point and bilateral force reflection is the primary performance objective, force-force control is commonly used. Bilateral force feedback for the exoskeleton will not only provide the necessary balancing, but will also allow the human operator to feel any contact with the environment.

ORNL's exoskeleton leg structure is fundamentally based on a "no knee contact" concept, where only sensing at the feet and the backpack is required. Based on past experience with the design and construction of teleoperators and the experience of other researchers in the area of orthotics, the mechanical interface with human tissues have indicated that pressures less than 2 psi can significantly damage skin and human tissues in a few hours. Long-term damage could include potential "pinching" of the tendons and deformation of the muscles such as those in the vicinity of the knee. Based on these observations and measurements, joint-to-joint motion tracking control of the knee utilizing displacement sensing would have difficulties in meeting the objectives of user comfort and non-impeding physical enhancement. To avoid these problems, a unique Cartesian-based control scheme based on force sensing at the foot and backpack attachments that will allow the exoskeleton system to closely follow the human body and thus avoid having to make a physical connection to the human knee and thereby prevent injury to the human operator has been selected by ORNL. The basic concept (again just for the leg) is shown in Fig. 1. The basis for the controls approach and the mechanical design concept are described in more detail in Appendices A and B. 


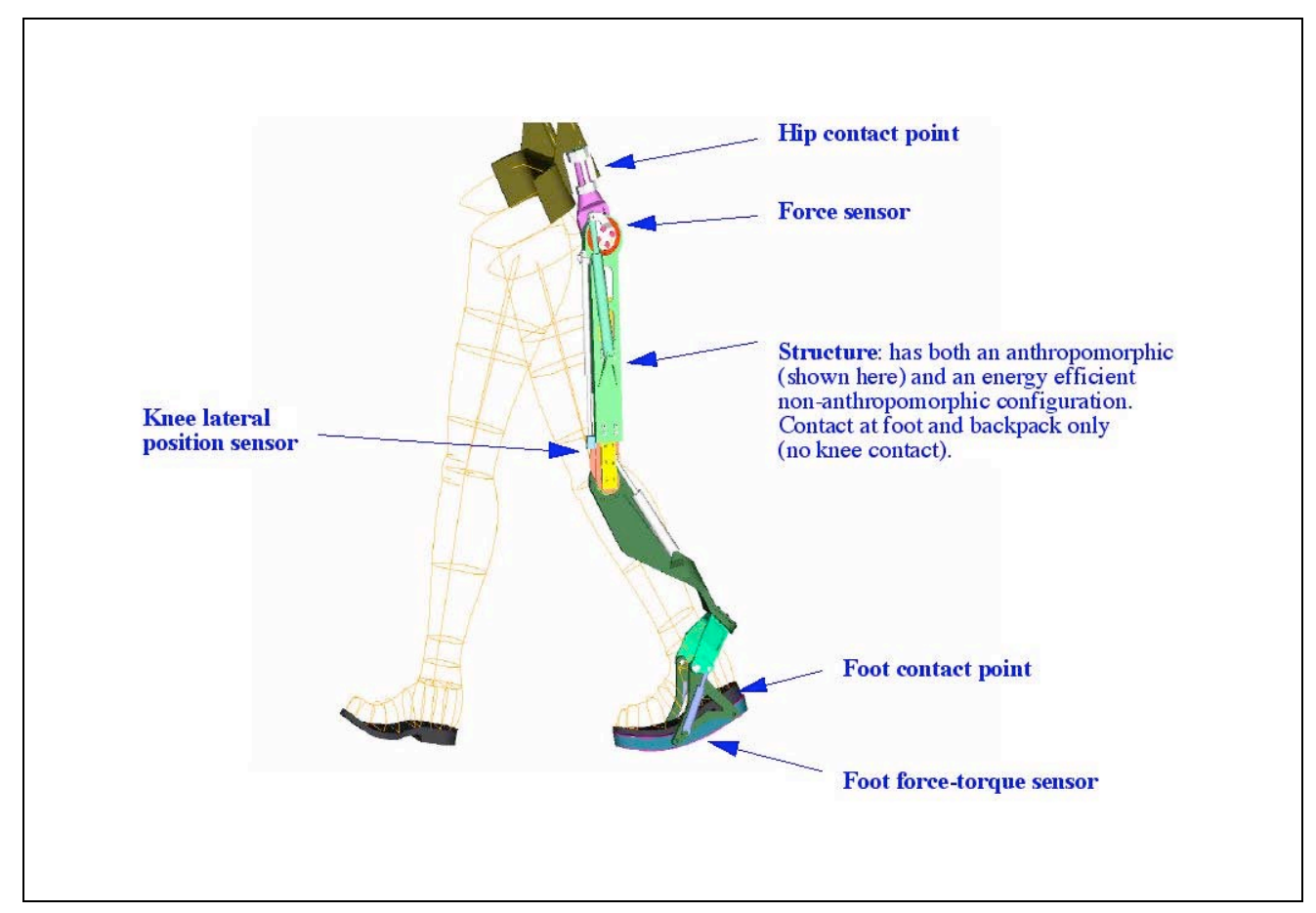

Fig. 1. Leg structure and sensing concept.

\section{QUASI-HYDROSTATIC CONTROL}

It was recognized that another control loop would be required to help reduce the energy loss of the system. While the demonstration of this outer loop was not scheduled until Phase 2 of this program, preliminary work was started on a way to efficiently control the energy from the hydraulic pump while still maintaining good overall tracking performance of the inner control loop. This approach is called quasi-hydrostatic control.

The duty cycle associated with an exoskeleton system changes significantly depending on the overall mission being imposed by the operator. In the past, fine hydraulic control has been achieved by means of a constant pressure hydraulic source utilizing a fluid control device such as a servo valve. Analogous to an electrical resistive element, a servo valve is notoriously lossy and can consume at least $1 / 3$ of the overall power generated by the pumping source. Since all of the teams currently working on exoskeleton development are using a hydraulic- or a fluid-based actuation scheme, fine control of hydraulic fluid without incurring significant energy loss is of paramount importance to this program. The quasi-hydrostatic control, a combination of flow control (to reduce losses) and pressure control (to achieve good tracking) has been explored to address this deficiency. The specific advantage of this approach is a large reduction in the peak power required from the pump. The challenge is that the force-force based control problem becomes very nonlinear. As an example, a comparison was made between a conventional constant-pressure control system and the quasi-hydrostatic approach on a single hydraulic cylinder by means of computer simulations. Figures 2 and 3 provide a comparison of the two approaches. The peak power from the constant pressure pump, after the initial transient, is $1,500 \mathrm{~W}$ whereas the peak power from the quasi-hydrostatic controlled system is $570 \mathrm{~W}$. The savings is even greater if we consider transients in the system (e.g., the initial transient in Fig. 2). 
Just as important, Figs. 4 and 5 show little difference between the two methods in terms of the tracking performance.

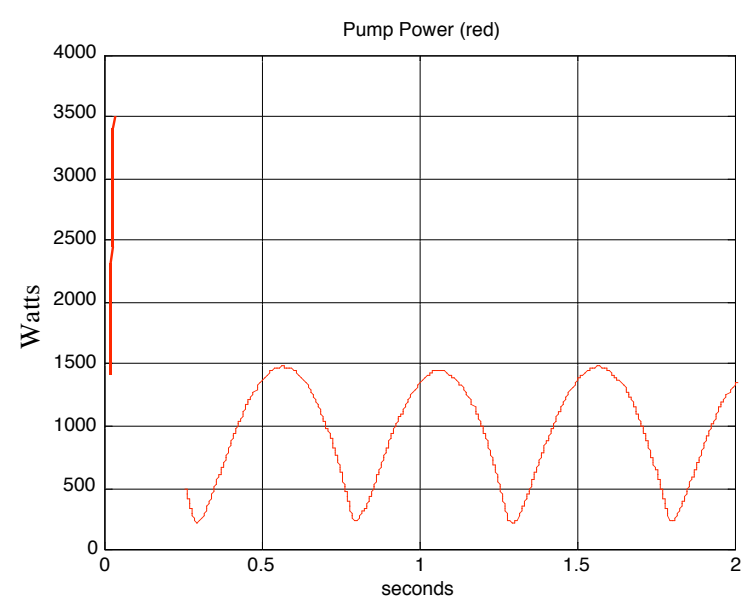

Fig. 2. Power requirement, constant pressure.

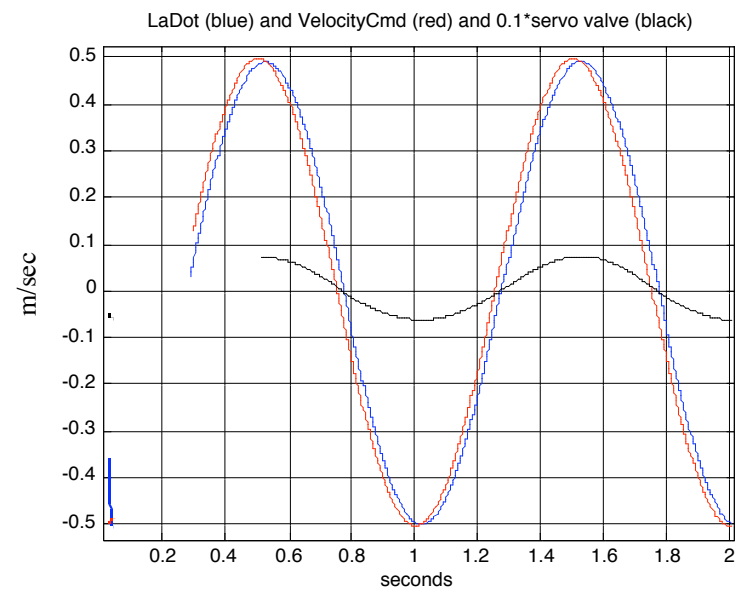

Fig. 4. Tracking, constant pressure.

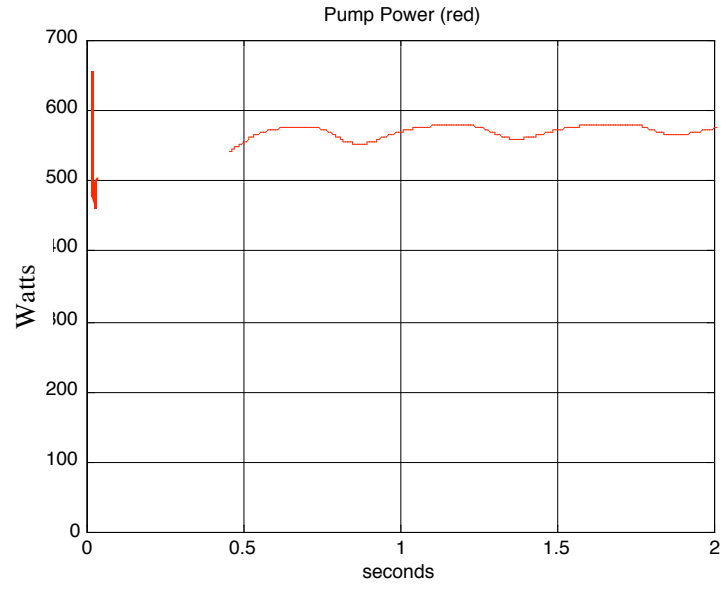

Fig. 3. Power requirement, quasi-hydrostatic.

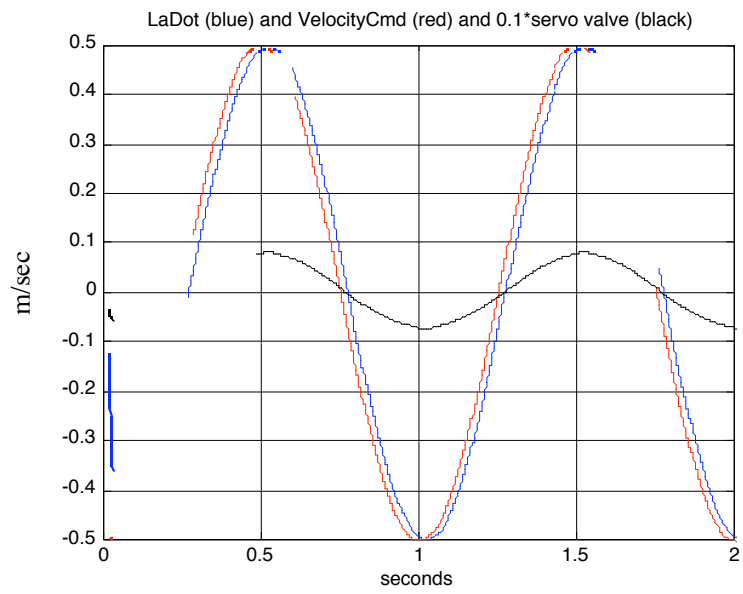

Fig. 5. Tracking, quasi-hydrostatic.

As can be seen from these simulations, a quasi-hydrostatic actuation methodology shows significant improvements in efficiency (maximum actuation power requirements cut in half) without sacrificing tracking performance.

\section{CONTROLS TEST STAND}

To demonstrate the control strategy before the construction of the actual exoskeleton, a highly instrumented test stand has been constructed (Figs. 6 and 7). The test stand has two major objectives stemming from the need to extend our understanding of both the biomechanical and engineering issues that will need to be addressed for the practical deployment of a fielded exoskeleton system. Specifically, from a biomechanics perspective, the purpose of this test stand was to: 
1) demonstrate and study force contact stability with the environment under sudden impact loads (e.g., foot fall), stumble recovery, hopping scenarios, varying environmental compliance, and backpack load variations;

2) demonstrate and study the overall force-based control strategy when coupled to a human operator;

3) minimize the force interaction between the human and the foot and backpack areas; and

4) investigate minimization of human machine interface induced fatigue by studying (a) the reduction of drag as a function of increasing the overall bandwidth (response time) of the actuator system, (b) the use of mechanical compliance to reduce impact forces on the structure, and (c) human and pack compliance requirements.

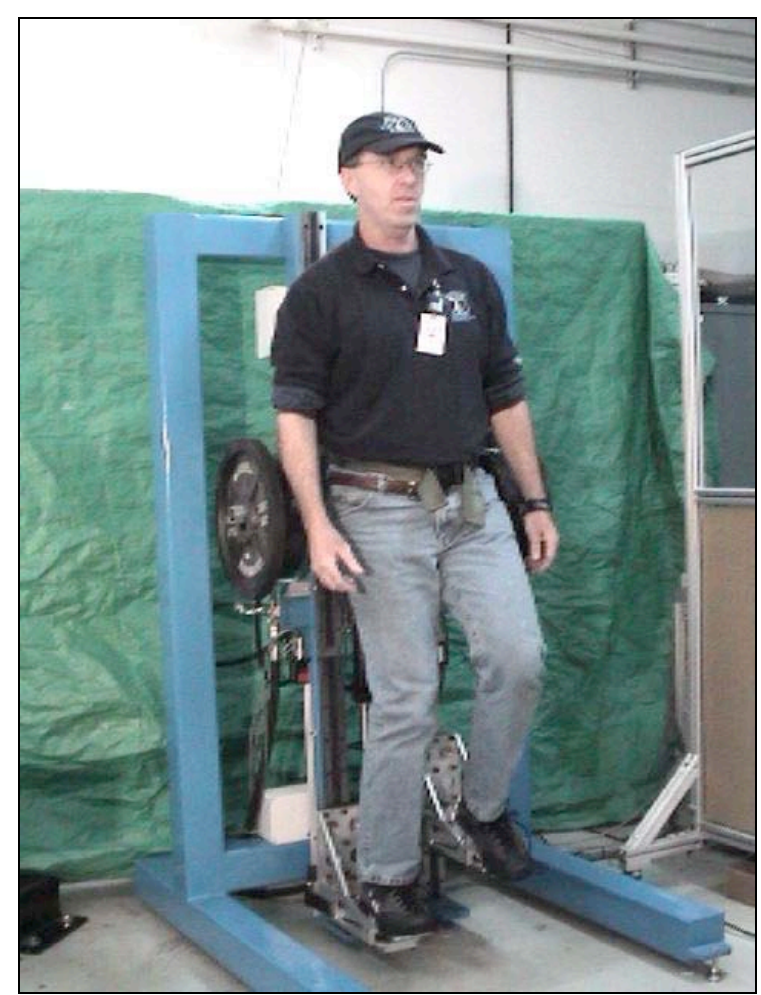

Fig. 6. ORNL's test stand.

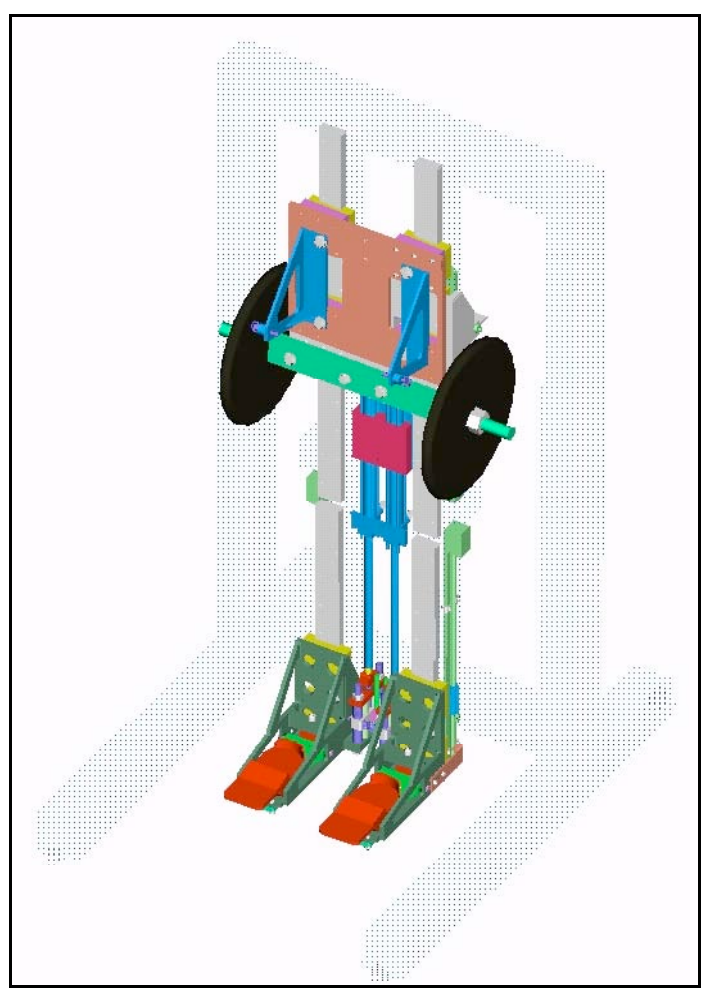

Fig. 7. ORNL's test stand physical layout.

From an engineering perspective, the purpose of the test stand was to:

1) understand the effect that mechanical compliance in series with the actuators (series elastic actuation) has on overall actuator bandwidth (during both small and large motions) and actuator saturation;

2) determine sensor requirements (e.g., dual force-torque sensing at the foot);

3) develop a methodology for tuning of controls settings;

4) minimize bias forces due to sensor degradation/creep; and

5) determine acceptable signal conditioning levels (e.g., dead band and bias loading of foot sensor in preferential direction) for all sensors. 
The exoskeleton test stand is uniquely instrumented to measure position, acceleration, and forces at both the feet and the backpack area and is vital to our understanding of all the issues mentioned above. Figure 8 shows the sensor location on the test stand. Data acquisition for the test stand currently can measure data with up to a $1 \mathrm{kHz}$ sample rate. The test stand has an open loop response in excess of $11 \mathrm{~Hz}$, making it almost transparent to the human operator, with the capability to exert mechanical forces of over $1,000 \mathrm{lb}$. Furthermore, almost any type of passive (and a restricted subset of non-passive) load can be generated and applied to the human operator.

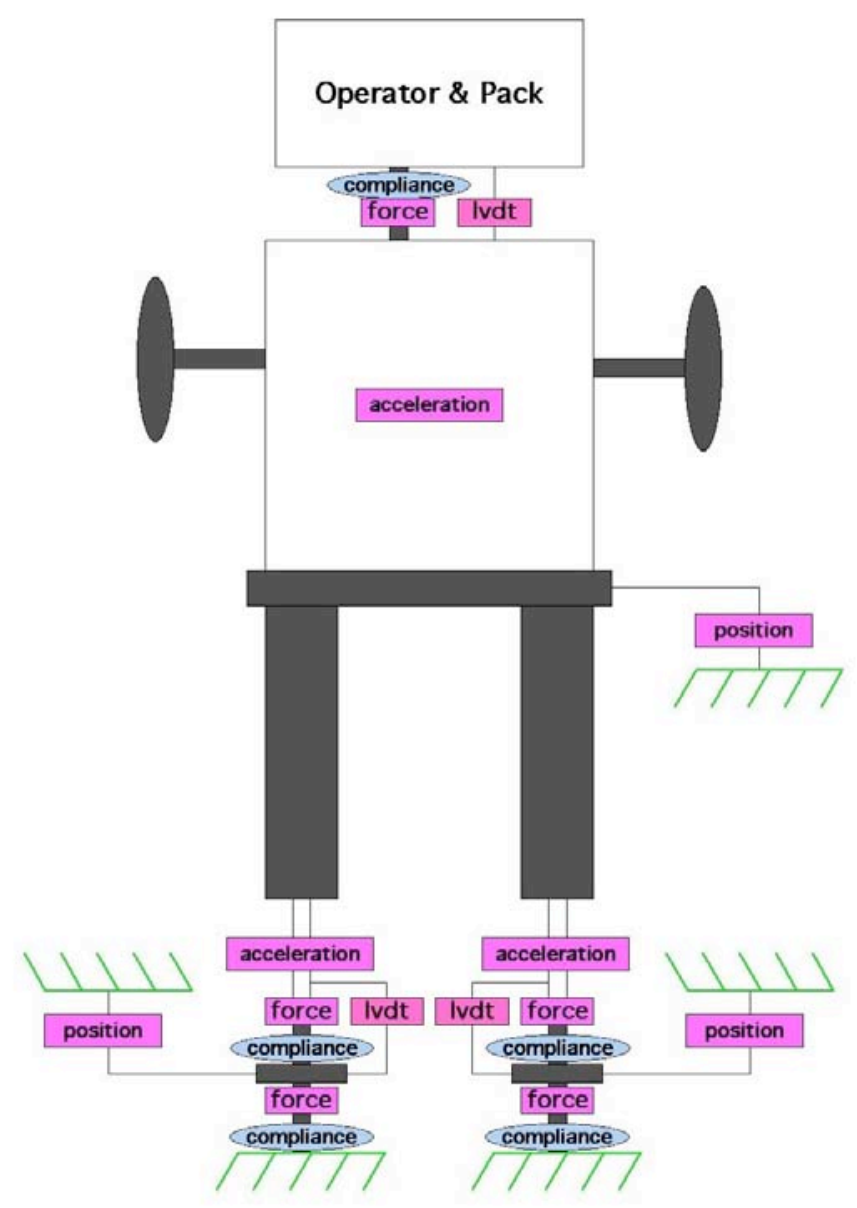

Fig. 8. ORNL's test stand sensor layout.

\section{TEST STAND RESULTS}

All of the Phase 1 goals have been met culminating in the demonstration of the force amplification on the test stand with adjustable gains up to 100:1 (Milestones 3.1 and 3.2) showing mass and inertia reduction control. Further, rapid foot contact stability was demonstrated completing Milestone 3.3. The exoskeleton controller approach has been implemented on the test stand and contact stability, low fatigue to operator, force amplification, and human interface at two contact points have all been demonstrated and documented on video which completes Milestones 5.1, 6.1, and 6.2. 


\section{FOOT FORCE-TORQUE SENSOR}

One of the most critical sensors in the proposed control strategy is the foot sensor (see Fig. 1). Since force sensing at the hip has to sense a limited number of degrees of freedom with relatively low impact loading, the foot sensor was the primary focus for the Phase 1 effort. The challenging aspect of this multi-axis force sensor is that it must conform to the bending of the human foot, withstand high impact loads (3x static loads), have better than a 2000:1 force sensitivity ratio, feel comfortable to the human operator, be integrated into a military style boot, and measure the forces on the human foot when either just the ball or the heel of the foot is in contact with the ground. There are no commercial force sensors that can meet such requirements. To fill this void, ORNL has relied on past experience in designing and building custom, high sensitivity force-torque sensors for high-precision applications as a basis for building a custom foot sensor.

\section{DESCRIPTION}

The new foot sensor utilizes two flexible plates that are constrained to maintain similar curvature. The top plate is in contact with the human foot while the bottom plate contacts the ground. The plates are thin and flexible enough so that normal foot flexing is permitted. The upper plate is attached to the human shoe through bindings so that all forces and moments generated by the foot are transmitted to the upper flex plate. The binding connection, which is tight at the ball of the foot, permits a small amount of fore/aft displacement between the foot sole and flex plate at both the heel and toe. This displacement allows normal flexing of the foot without stiffening the boot.

The upper flex plate is connected to the lower (ground contact) flex plate through a number of vertical and horizontal force sensing elements. These connections are designed to transmit the forces to the sensing elements in their sensitive axis while not imposing loads in unmeasured directions. The design of these connections is very important to the overall accuracy of the foot sensor system. A key advantage of this design is that the number of required force sensing elements is reduced since the flex plate carries all the transverse loads (lateral and fore/aft), thus enhancing flexibility of the sensor and allowing for a more compact design.

The first prototype foot force-torque sensor is shown in Fig. 9. This is a 4-axis unit developed specifically for an exoskeleton, including bosses to connect to the exoskeleton ankle frame and a vertical load rating of $1200 \mathrm{lb}$. This design may be easily adapted to include more axes and for human biomechanical measurement applications (which would not require the $1200 \mathrm{lb}$ vertical load). The Z-axis is defined as perpendicular to the flex plate (corresponds to vertical when standing on level surface) and the $\mathrm{X}$-axis as horizontal pointing to the side with the longitudinal Y-axis pointing to the front (Fig. 10). The prototype foot sensor has four Z-axis force sensors (two at the ball and two at the heel) from which total Z-force (Fz) can be computed as well as $\mathrm{X}$ and $\mathrm{Y}$ moments $(\mathrm{Mx}, \mathrm{My})$. A force sensor along the Y-axis measures fore/aft force (Fy). Figure 10 shows the general arrangement of the strain gage bending beam force sensing elements (see Fig. 11). Figure 12 shows a view of the sensor in the flat configuration (it is 1.75 inches thick including the 0.5 -inch rubber padding on the bottom). 


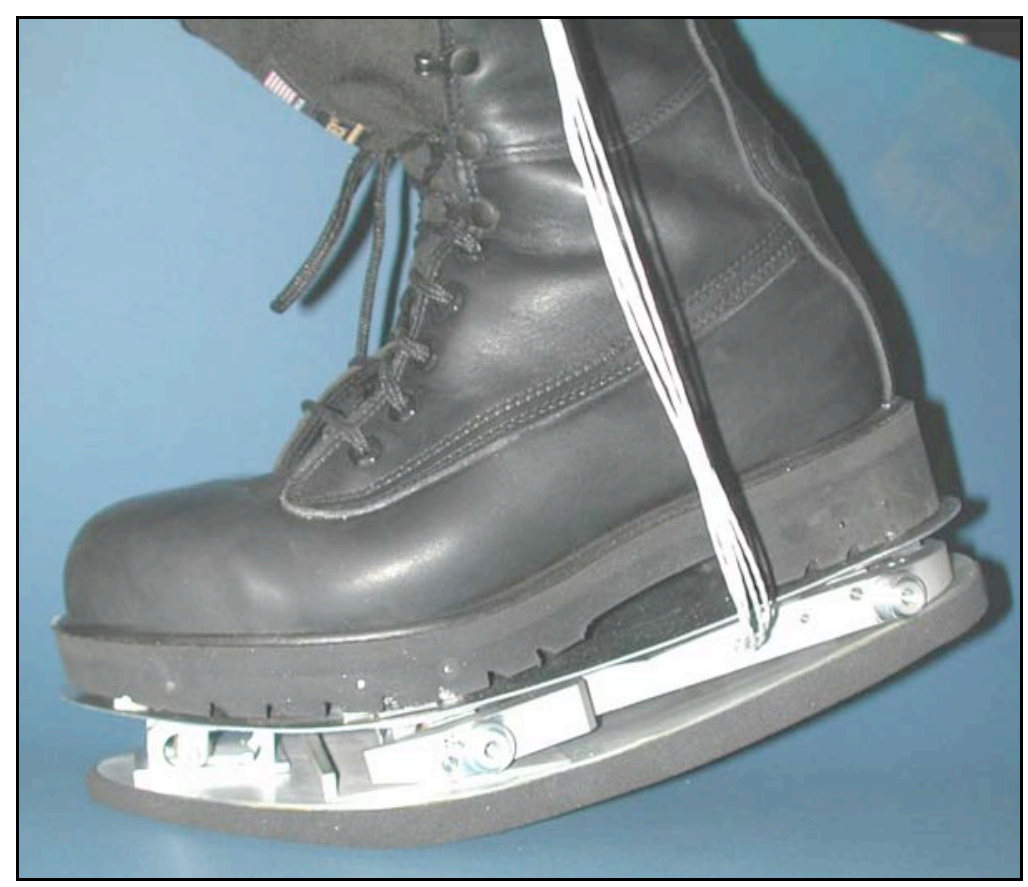

Fig. 9. Foot force-torque sensor attached to boot.

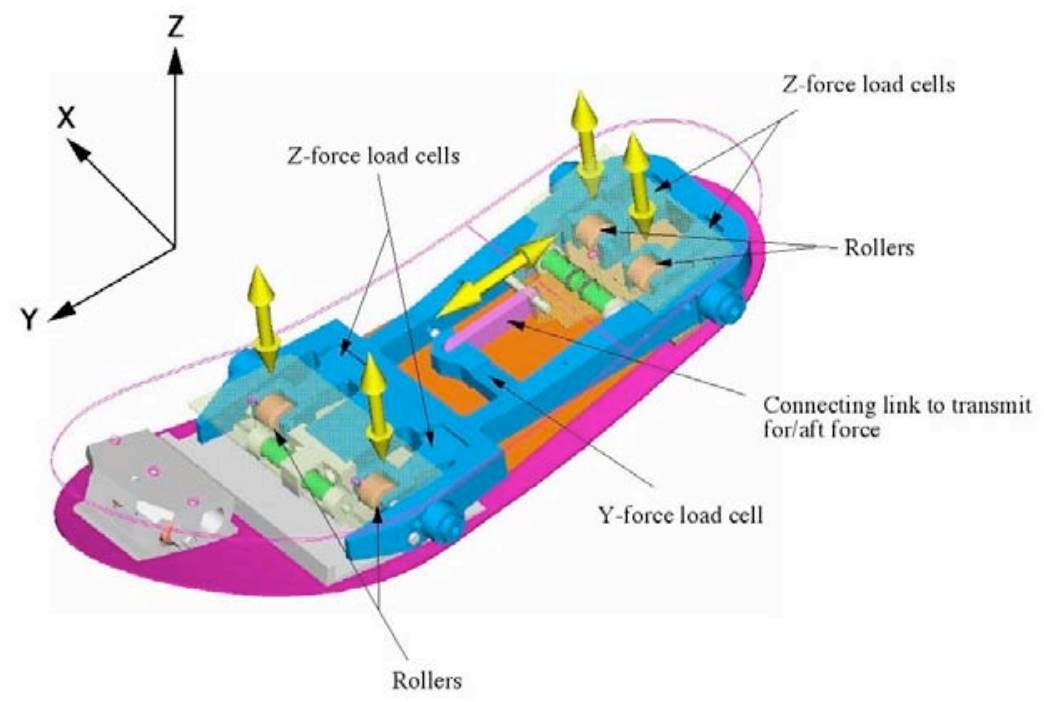

Fig. 10. Sensor force measurements.

In this design, considerable translational displacements occur between the upper and lower flex plates. These relative motions occur primarily in the Y direction and are accommodated by the connections between the upper flex plate and the force sensors that are mounted to the lower flex plate. Z-direction loads imposed on the flexible foot input plate are transmitted to the rigid load sensors through rollers (Fig. 10) that allow significant angular and Y translational motion (with little resistive force) while maintaining stiffness in the load sensitive Z-direction. 
Figures 10, 11 and 13 also show the lateral constraint elements that are used to minimize lateral forces on the vertical load cells. This helps assure accuracy of load measurement. The lateral constraint elements behave like ball bearings so that nearly friction-free flexing and translation of the flexible input plate is permitted without lateral movement.

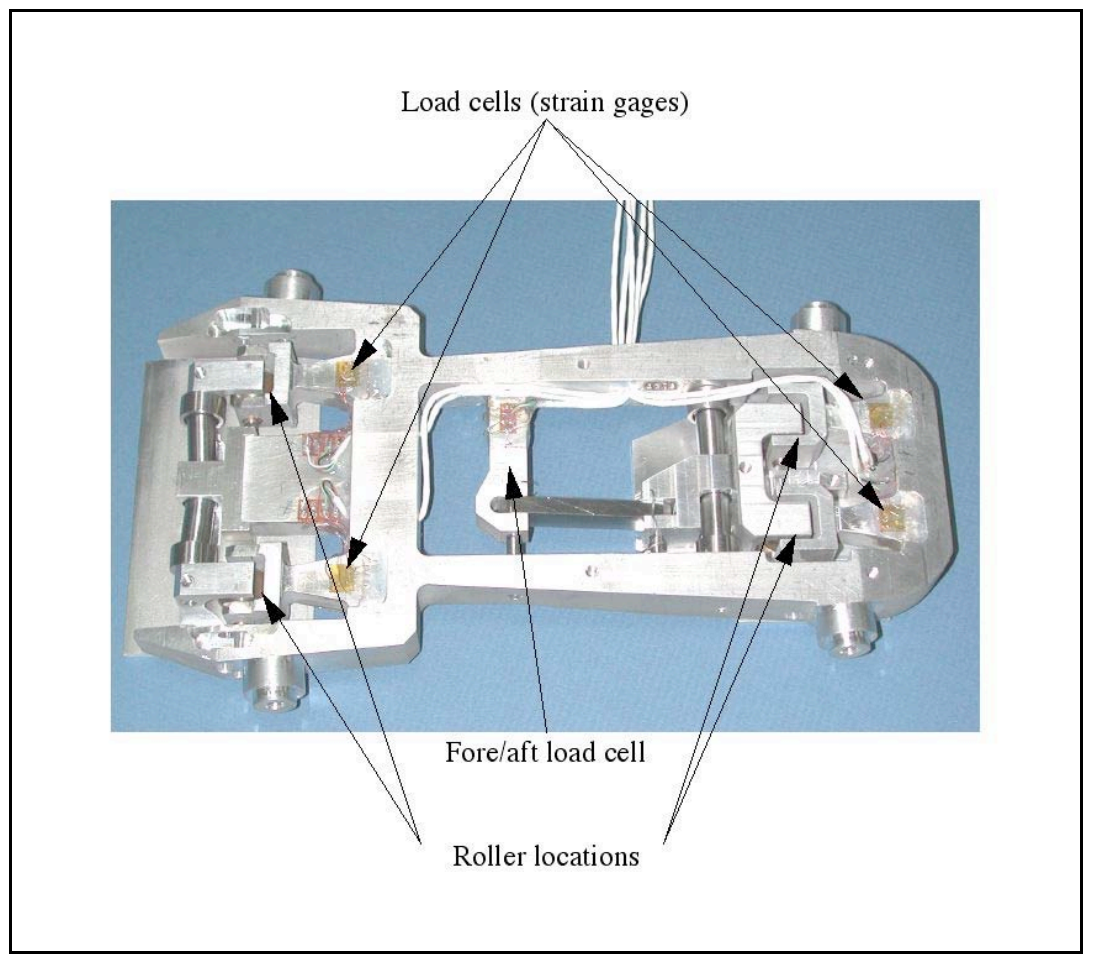

Fig. 11. Prototype foot sensor load cell details (bottom view).
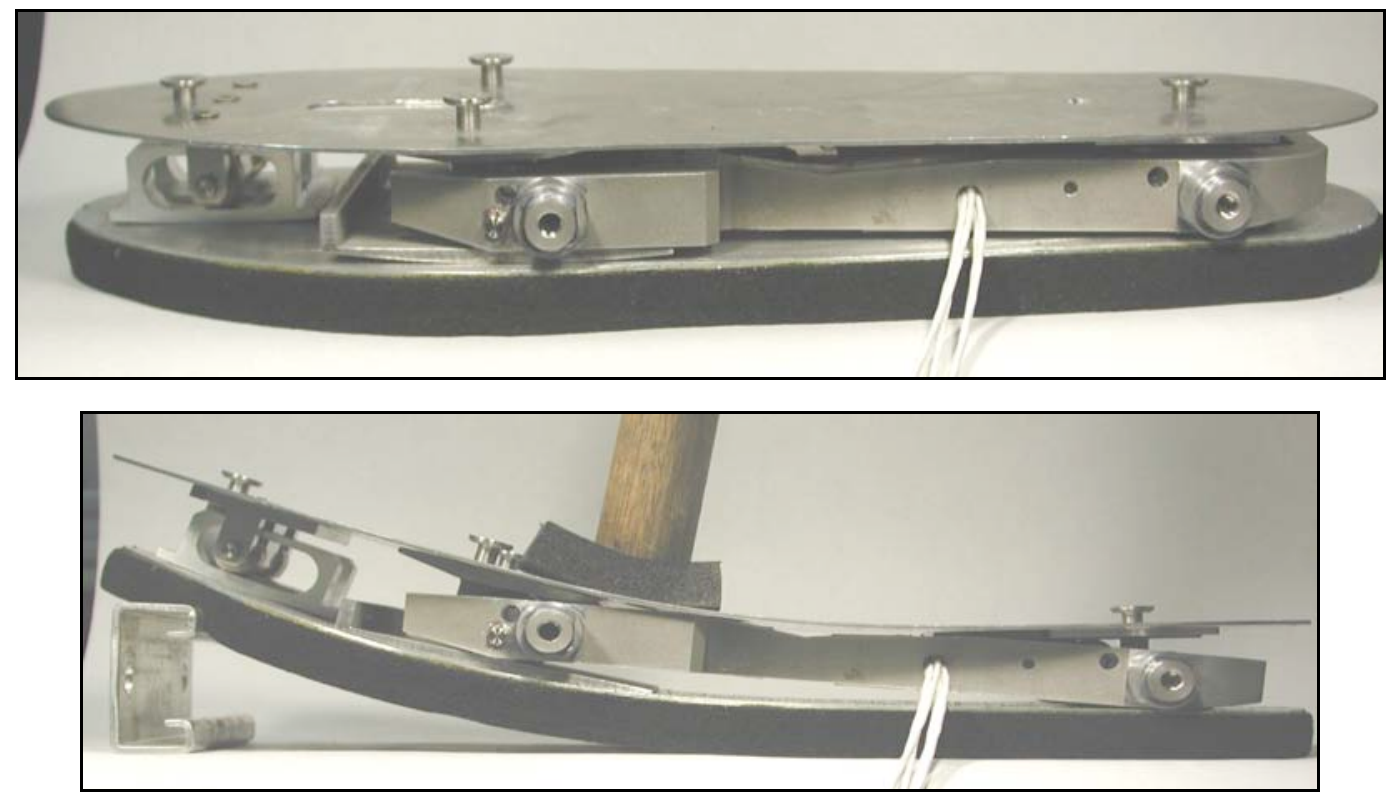

Fig. 12. Force-torque sensor shown flat and flexing. 
Bending of the upper flex plate creates loading on the load cells that is not exactly parallel with the sensitive axis. This off-axis force (while small) is not measured but can be computed if the off-axis angle is known. Future versions will include a sensor to measure the degree of bending in the upper flex plate allowing the off-axis components of force created by the angled contact to be calculated and factored into the output results.

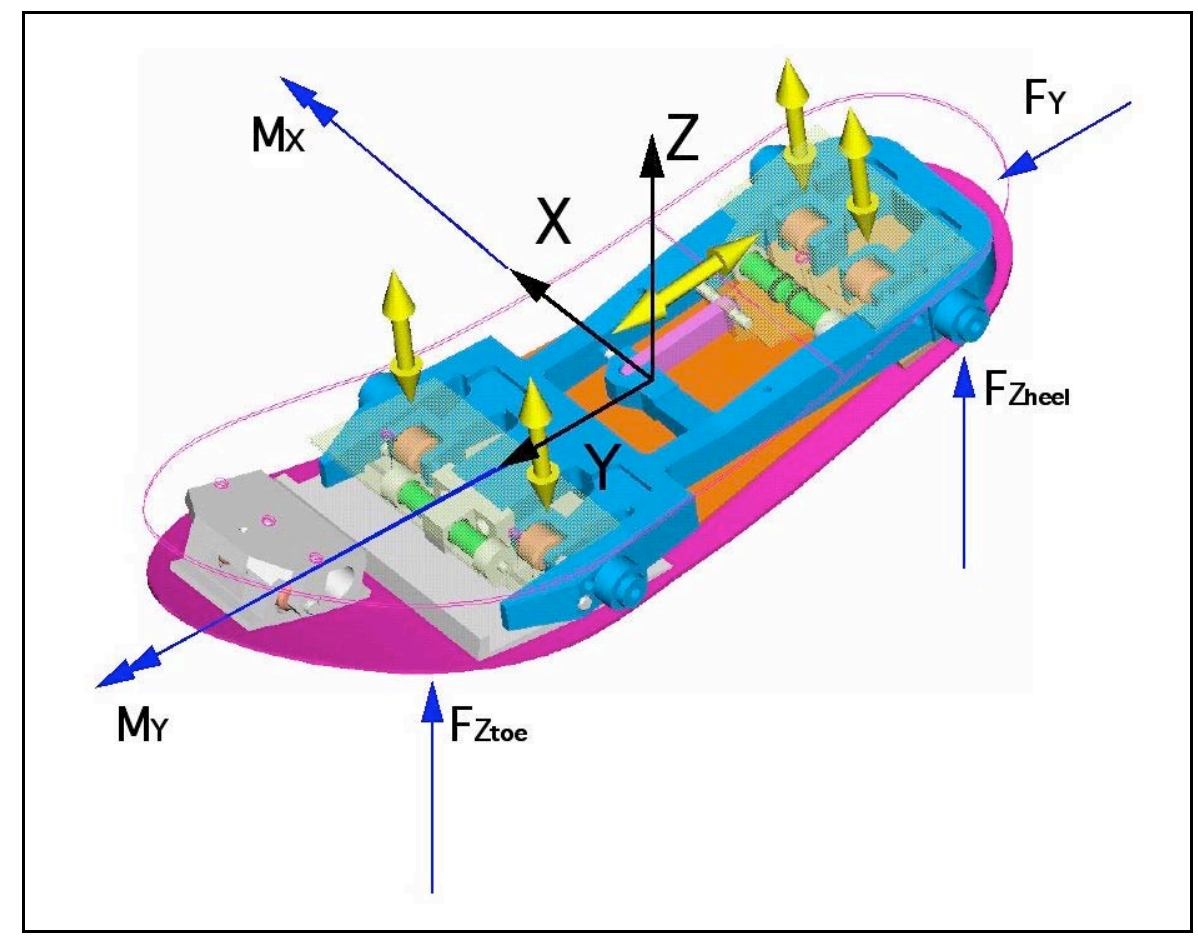

Fig. 13. Measured forces and moments.

The current design does not measure lateral horizontal loads (not a requirement for the exoskeleton application). To add this capability would require the addition of two lateral load cells and linkages or shear pads to transmit the lateral loads. Figure 14 shows a conceptual model of a 7-axis sensor. This design shows ball joint linkages that transmit loads only along their centerline. The ball joint ends are required to accommodate the slight motion that occurs when the top plate flexes. It also shows an optional toe force sensor. Flex angle measurement would also be required to calculate the vector angle of the toe force. 


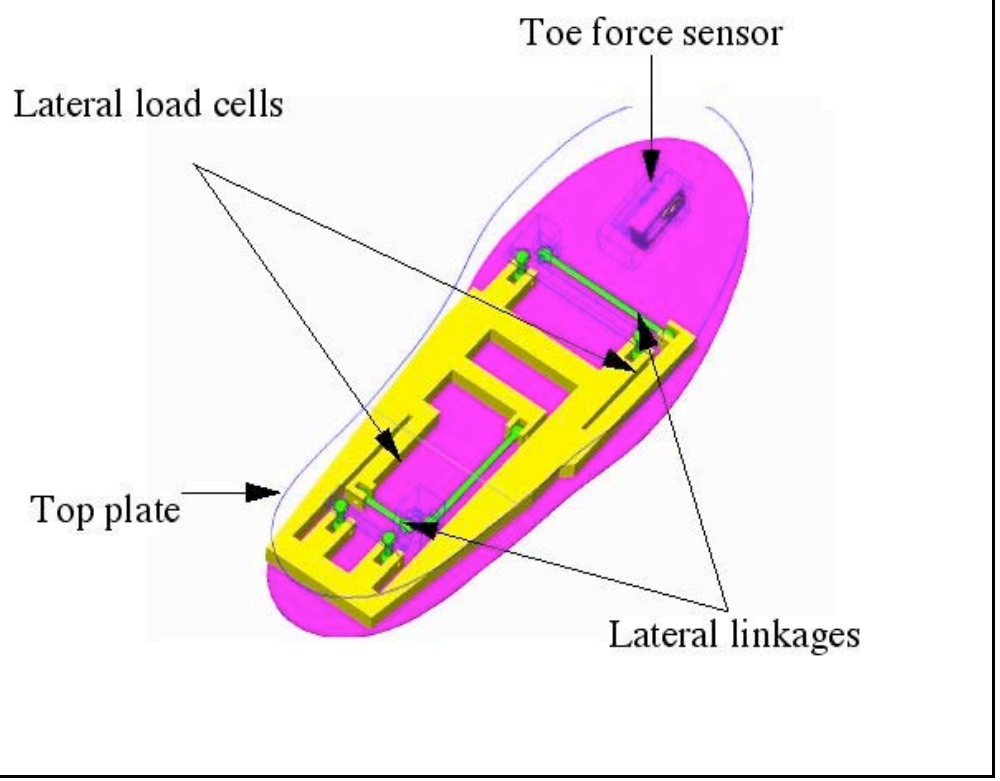

Fig. 14. Conceptual 7-axis foot sensor.

\section{FOOT FORCE-TORQUE SENSOR TESTING}

After calibrating the individual load cells, data was collected from the sensor during standing, walking (slow and fast), jumping and jogging. From the five load cells it is possible to measure total vertical (Z) force, ball or heel forces, moment as viewed from the saggital plane, moment as viewed from the frontal plane, and fore/aft horizontal force. Figures 15 through 20 show typical data collected in these activities. The foot sensor data compares favorably with published force data that has been taken from commercial, stationary force plates.

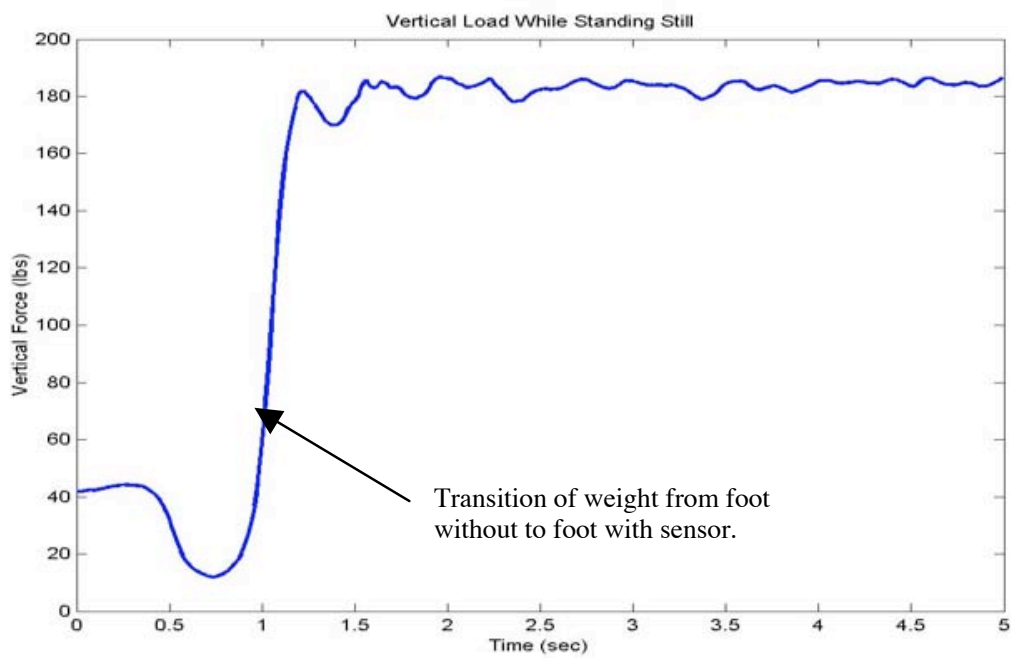

Fig. 15. Standing force data.

(Note variation caused by balancing action of $185 \mathrm{lb}$ subject). 

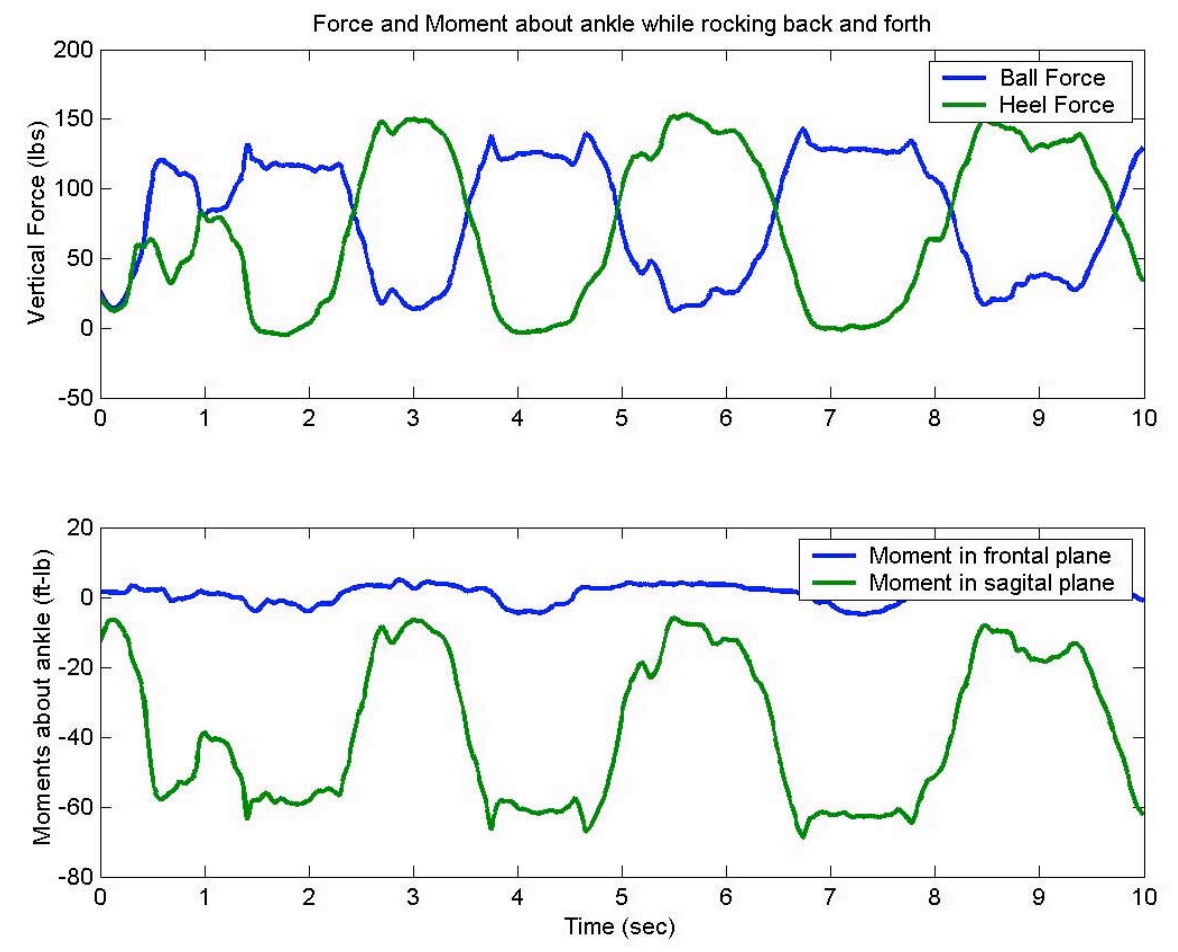

Fig. 16. Standing/rocking force data.
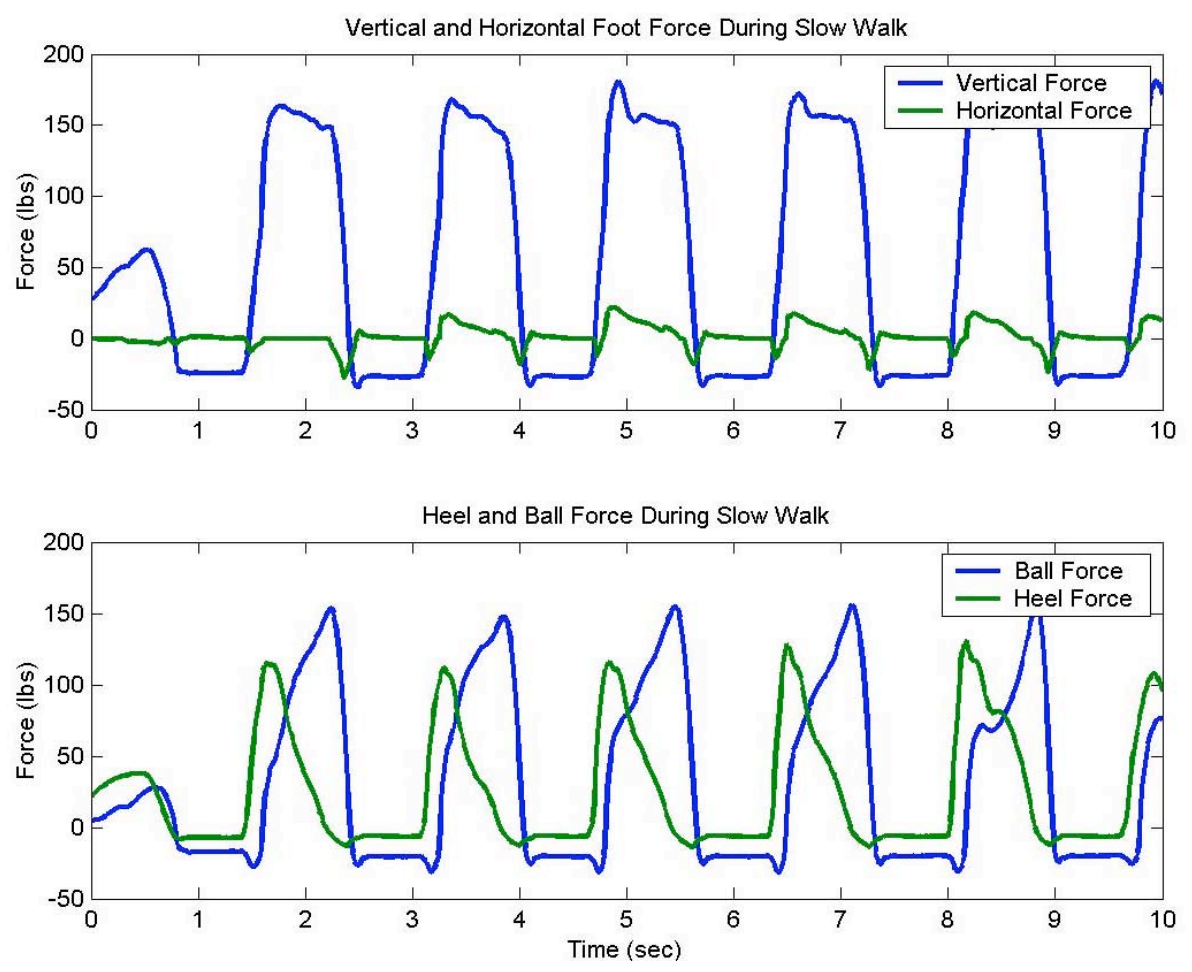

Fig. 17. Slow walk force data. 

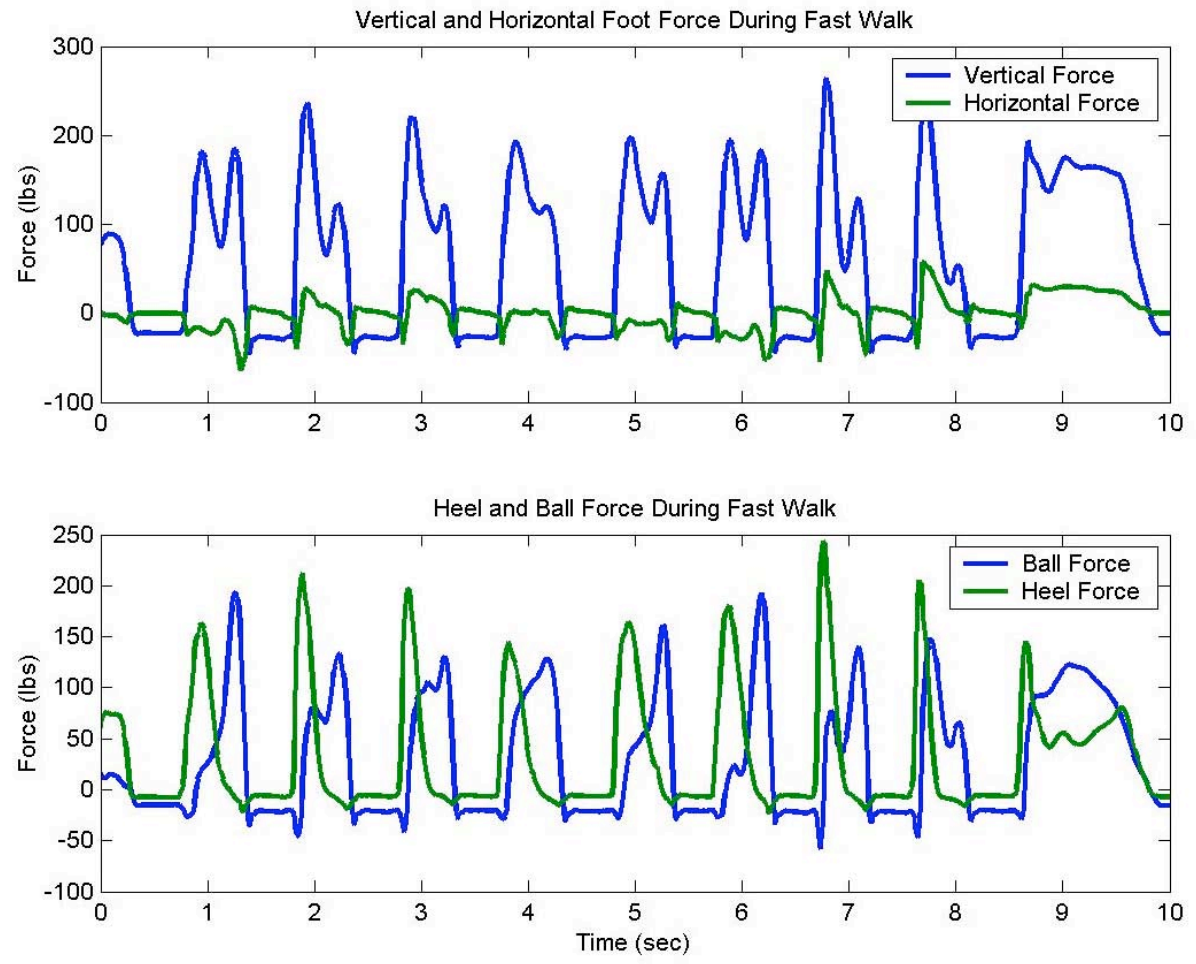

Fig. 18. Fast walk force data.

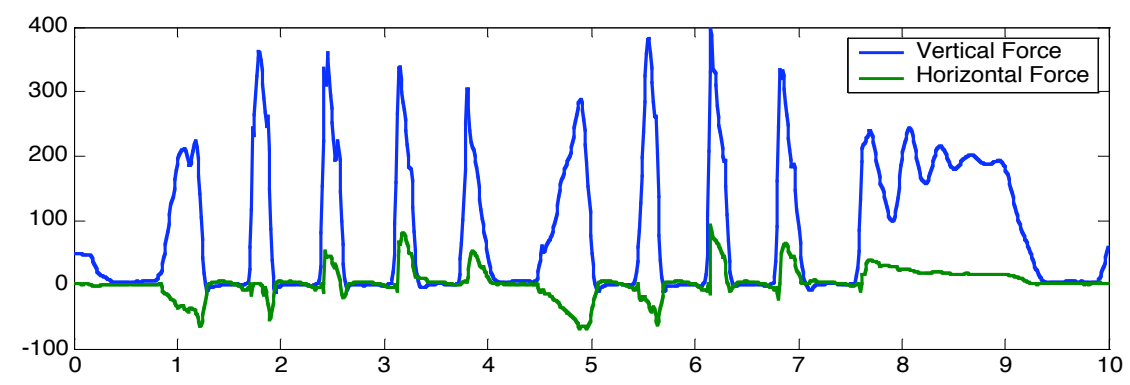

Fig. 19. Jogging force data.

While initial reaction to wearer comfort appears very positive, metrics as to the comfort of this sensor from a soldier's perspective are not yet available. The Army Research Lab (ARL) is currently performing biometric analysis on the effect of an extended sole on soldiers' performance during various tasks such as marching, squatting, and running that will help in quantifying the human response to this sensor. 


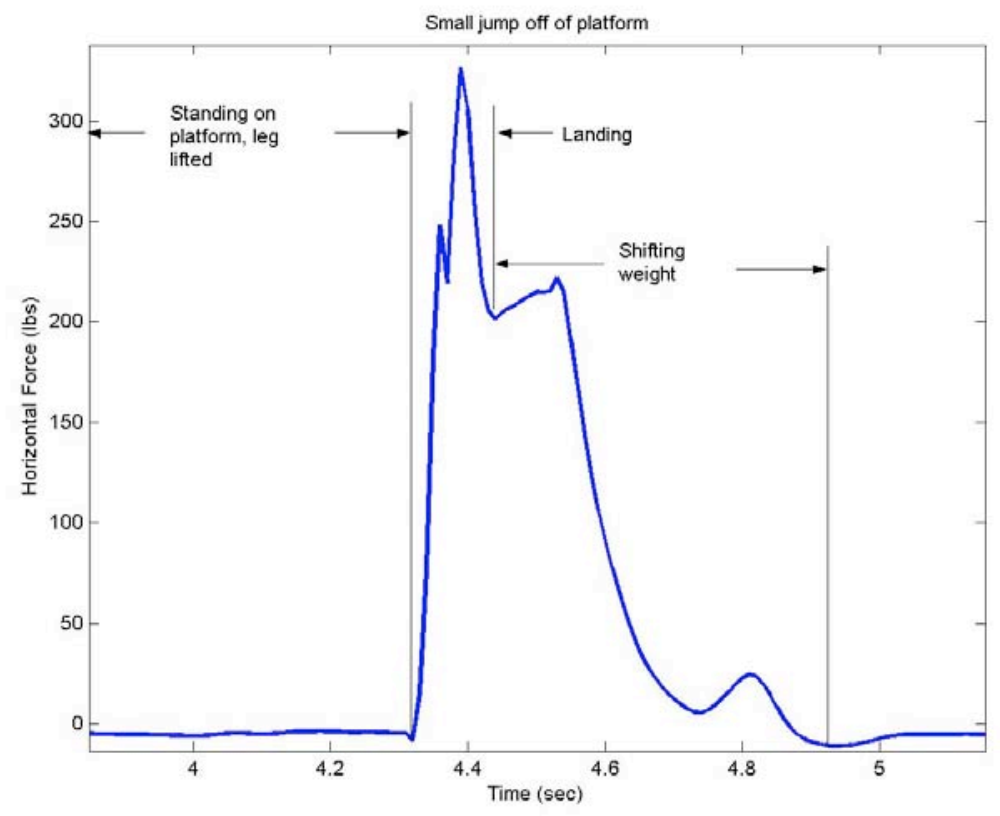

Fig. 20. Jumping force data.

\section{SUMMARY}

A foot sensor has been developed at ORNL for an exoskeleton application. The sensor is based on robust foil strain gage technology with five integral bending beam load cells. The sensor is lightweight $(2.5 \mathrm{lb})$ with a low profile (1.75 in including bottom tread) and is designed to interface directly with a typical boot (with minor modifications). Mechanical bosses have been designed to allow ready integration into the exoskeleton support structure.

The sensor is rated for a maximum vertical load of $1200 \mathrm{lb}$ but has a sensitivity of $0.16 \mathrm{lb}$ in the vertical, $0.08 \mathrm{lb}$ in the horizontal directions, and $0.1 \mathrm{ft}-\mathrm{lb}$ moment about the ankle. The vertical sensitivity is 7500:1 (i.e., 1200/.16) which is outstanding for a multi-axis force sensor and is achieved by exploiting the entire geometry of the sole volume.

The sensor provides data similar to larger and more expensive force plates with the addition of moment about the ankle roll motion. It is unique in that it can measure vertical forces at the ball or heel of the foot. It conforms to the human foot and appears to be extremely accurate simply because it exploits the full geometry of the sole of the boot. The sensor can be easily scaled either up or down to accommodate the force impact loading. The foot sensor is comfortable to wear, superior to commercial force-torque sensors, unique in design, and will meet the expected stresses encountered by the exoskeleton environment. The design provides space inside the sensor for integration of all necessary signal conditioning electronics. The sensor is readily sealable to prevent moisture and environmental contaminants from entering the device. 


\section{SOFT TISSUE INTERFACE}

\section{INTRODUCTION}

A powered exoskeleton works in intimate mechanical contact with its human user. The design of an effective exoskeleton requires extraordinary attention to the details of this coupling. A wellengineered exoskeleton with compact, efficient power sources, stable control, and dexterous motion can still fail if it does not mesh seamlessly with the user's mobility, if it applies unusual or excessive forces to the body, or if it loads the body in a manner that causes skin irritation or discomfort. The design of an effective human interface has challenged every tool and machine that works in contact with the body, from shoes and boots, to clothing, to prosthetics, to orthotics. The challenge is no less for powered exoskeletons.

Human interface considerations divide roughly into two categories. First are issues related to control and haptic interactions between machine and human. Here, one must pay attention to the dynamics of the control system, where sensors are located and what quantities are being measured, and the dynamics, kinematics, and human motor control of normal posture, motion and gait.

Second are issues related to contact forces, moments and pressures exerted by the machine on the human. Here, the critical factors are the load duration characteristics of the shear and normal forces applied to the skin and bony prominences. Body locations vary widely in their ability to withstand external forces. For example, the heel can tolerate large, steady normal forces and substantial impact loading as it does daily during standing and gait. Bony prominences can only take small normal forces over brief periods of time before blood is squeezed out of the area and the skin starts to break down. Fleshy areas over muscle bellies in the shank and thigh can take a reasonable level of normal load, but offer a highly compliant coupling to the underlying skeleton rendering close, collaborating control between exoskeleton and human skeleton almost impossible. No area of the skin can tolerate shear loads for any reasonable period, which means the direction of the force loading vector applied by the exoskeleton to the body must be known and result from careful design.

All of these considerations regarding human-machine coupling have been known and studied for decades in the design of shoes, athletic footwear, high performance hiking and climbing boots, backpacks, prosthetics and orthotics. Little is known, however, on how these considerations change for active exoskeletons.

For the ORNL exoskeleton human couplings, we developed preliminary concepts and designs while being guided by results from traditional biomechanics research. In parallel, we developed a test plan with the intent of following an informed iteration process as the design was refined. Our objective is to have the exoskeleton fit be equivalent to the fit of a high performance hockey skate. Our target was to achieve "tolerable comfort." Like orthotics, skates must be closely coupled to the body, and must transmit considerable loads between the body and the ground. Skates are not as comfortable as bedroom slippers, but if properly fit, can be worn for long

periods while meeting performance requirements. The normal and shear loading of the skin and 
underlying anatomical structures by the exoskeleton, however, must be kept within safe limits for all proposed mission tasks.

The remainder of this section briefly reviews relevant prior art in biomechanics research then details the designs for the human couplings

\section{PRIOR ART}

\section{Lessons from Biomechanics and Anthropometrics}

The dimensions and clearances for the exoskeleton structure were dictated by human anthropometrics. Tabular information on 5-95\% body dimensions for adult males was acquired from Woodsen et al. (1992). Human joint range of motion data was taken from Barter et al. (1957) to determine design requirements for the exoskeleton motion and for the placement of mechanical limit stops for safety. The details of human joint motions during gait were taken from Rose and Gamble (2000) for use in designing the exoskeleton degrees of freedom.

Biomechanics studies that measured the frequency content of gait were used to determine the closed-loop frequency response of the exoskeleton system. Studies show that $90 \%$ of the signal power of human limb motion during gait is $6 \mathrm{~Hz}$ or less (Antonsson and Mann, 1985; Winter, 1990). Most of the force signal content during heel strike is $10 \mathrm{~Hz}$ or less, but there are some impulsive loading components during shod walking that can reach $75 \mathrm{~Hz}$ (Harris et al., 1996). The addition of padding between the foot and the exoskeleton will filter high frequency impulsive forces but because the exoskeleton controller must be stable for high frequency impulsive disturbances caused by jumping or stomping, it was determined that its closed-loop response should be $10 \mathrm{~Hz}$.

\section{Lessons from Pressure Sore Studies}

The physical attachment of machine to human must be designed carefully. There is considerable knowledge and experience embedded in the fields of orthotics and prosthetics which for decades has tackled problems of applying significant forces to the skeleton while keeping skin loading to acceptable levels.

Abnormal loading of skin causes tissue breakdown leading to pressure ulcers. Some adaptation ("skin toughening") is possible through training programs, but the more appropriate solution is to keep loads within safe limits. One problem with exoskeletons is they apply loads in locations on the body that are not intended to carry loads of any magnitude for long duration. The classic example are skin ulcers on the buttocks of individuals with spinal cord injury who are seated in wheelchairs for long periods and who forget to shift their weight. Another example is the skin breakdown that can occur at the socket-skin interface of a prosthesis for above-knee amputees. Practically everyone has experienced a minor example of abnormal loading from wearing poorly fitted shoes. An overview of the skin's response to loading can be found in the review by Sanders (1995).

The literature on acceptable skin loading is sparse, but it is clear that skin breakdown and ulcer formation is a function of load magnitude and duration. While definitive load-duration 
thresholds have not been established for human skin, guidelines have emerged from the results of several research projects using animal models or human subjects and those guidelines have been translated into clinical practice for the prevention of pressure sores and design guidelines for orthotics and prosthetics (Sanders et al., 1995). The generally accepted model is that surface tissue pressure leads to ischemia that in turn leads to tissue damage. Reasonable estimates of critical loads are $100 \mathrm{~mm} \mathrm{Hg}$ ( 2 psi) for two hours or $35 \mathrm{~mm} \mathrm{Hg}$ for four hours. The implication for the exoskeleton is that modest attachment pressures can be tolerated for long periods while high pressures will lead to skin breakdown.

Tissue damage occurs first in deep tissue. Damage will occur to the muscle before seen on the skin. Poorly designed exoskeletons may cause damage to the user before any external symptoms are noticed. The location of the applied pressure impacts damage. Pressure applied where the bone is near the surface will rapidly break down skin because it is easier to squeeze the blood away from the region. Exoskeletons with attachments at bony prominences in an attempt to couple closely to the skeleton are sure to fail.

Shear loads are equally important although less well understood. Shear loading is particularly disruptive for skin, but a small shear load is acceptable even over many cycles. The friction of the material against the skin has a role in the damage process (Sanders, 1998). It appears that skin tolerates high cycles of low shear loads better than few cycles of high loads which bodes well for an exoskeleton designed with low shear loads which cycle with each step.

Moisture and temperature influence the ability of skin to maintain integrity under load. Our attachment designs will keep the skin dry and cool, through the use of appropriate padding materials drawn from orthotics practice. The exoskeleton attachment points must also be kept away from heat generating sources.

\section{Lessons from Prosthetics and Orthotics}

The physical architecture of the exoskeleton is more related to orthotics than to prosthetics. Classical design of orthoses is covered in several clinical textbooks, including Bunch (1985), Nawoczenski (1997), and Redford (1986). Also, a brief review of powered orthotics is provided in Goldfarb (1996). There are a few examples of full-leg orthoses (hip-knee-ankle-foot orthosis [HKAFO]), mostly designed for paraplegic walking systems. The hip guidance orthosis (HGO) was developed in Oswestry, England. The reciprocating gait orthosis (RGO) is a similar product developed at Louisiana State University. Both provide guidance at the hip and knee joints and couple the hip joints for reciprocal motion. Neither has met with widespread clinical success because of their bulk, weight, and difficulty in donning and doffing. One criteria for the exoskeleton is that it can be abandoned rapidly in case of emergency. This will be challenging for designs which transfer power to the body, but simpler for machines which walk along with the human.

There are many examples of commercial knee orthotic braces that are both functional and comfortable. Many athletes wear them for several weeks after sustaining a knee ligament injury. For example, the DonJoy product line of knee braces from Smith and Nephew provide a comfortable yet secure fit to the thigh and calf. Likewise, ankle orthoses designed to limit 
motion have been successful in the commercial market and are designed for all-day wearing. Products from Air-Cast, Smith and Nephew and Active Ankle systems provide good design models for the exoskeleton.

Our attachment point designs draw directly from tested designs used in orthotics. To reduce costs, wherever possible we take advantage of off-the-shelf orthoses and athletic equipment.

\section{COUPLING ISSUES}

The fundamental concept of the exoskeleton is close operation with, but not exact correspondence to the human skeletal structure. An advantage of this approach is that the exoskeleton attaches to the user only at the foot, waist, and shoulder, which greatly simplifies the task of designing attachment points. A second fundamental concept of the exoskeleton is to couple to the user through force sensors. The controller acts to minimize the loads seen by the force sensors, which results in a gentle coupling to soft tissues.

As with any orthosis, the challenge in designing attachments is the trade-off between rigid coupling to the skeleton and comfort for the user. Because rigid coupling is not feasible, any connection will move relative to the skeleton. Our preliminary designs for attachments minimize this shift to be within the stability limits of the controller while still retaining tolerable comfort for the user. A well-designed attachment point meets these requirements: (1) distributes the load, (2) minimizes shear, (3) avoids bony points, and (4) keeps the skin cool and dry. Another critical design criteria for the attachments are that the exoskeleton be easily donned and doffed.

\section{Foot Interface}

The most challenging attachment point is at the foot because space is tight and because the boot needs to incorporate the force-torque sensor. The interface design consists of a boot with the rigid force sensing element sandwiched between the tread components that contact the ground and the sole components that contact the foot. Each component has several layers to satisfy the multiple functions of comfort, flexibility, and accurate force sensing.

Figure 21 shows the stack up of layers at the foot from the ground to the skeleton. Each layer has an essential purpose and both control and comfort are effected by the entire impedance chain. For excellent control, a tight coupling to the skeleton is needed. If control was the only issue, the sensor top plate would be connected directly to the skeleton by bone screws, but this of course is not possible. Because of inherent compliance in soft tissue, the boot can be padded with layers of comfort material that have approximately the same compliance as the tissue in the foot. Compliance between the sensor plate and the skeleton is easier to handle in the controller than a deadband because compliance can be modeled and is relatively well behaved. To eliminate deadband, the boot padding will conform closely to the foot when the boot is closed.

The foot coupling must handle bi-directional forces along the vertical axis because as the foot leaves the ground during the start of the swing phase, the exoskeleton must follow along. Unlike a regular shoe, the boot will be padded under the closure straps and laces as well. 
The user effectively is "standing" on the foot plate of the exoskeleton with the 6-axis force-torque sensor fitting in series between the human and machine. Thus, the user is raised off the ground, much like wearing boots with very thick soles. The height off the ground is dictated by the height of the force-torque sensor and related fittings. The target package height specification for the sensor is $<4.5 \mathrm{~cm}$. Testing will be done with standard boots attached to sensor mockups to ensure that normal gait is achievable with this configuration.

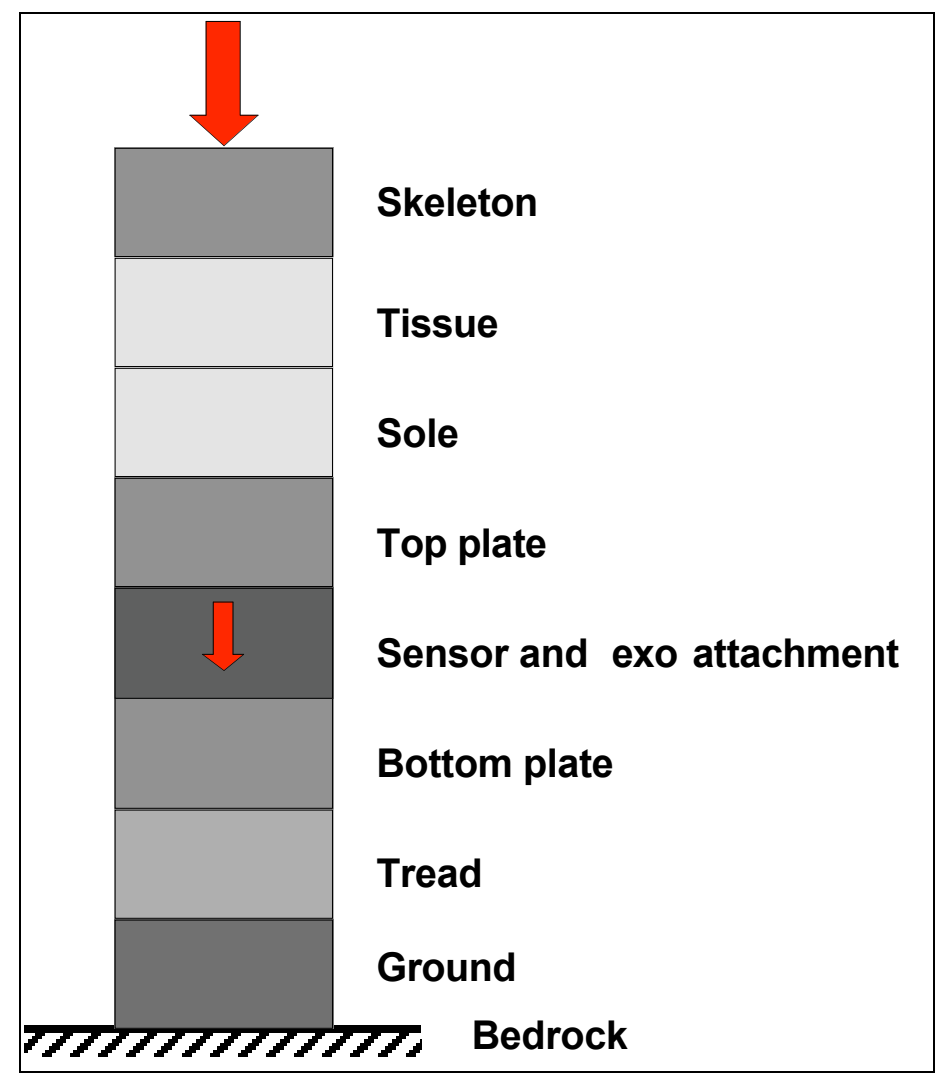

Fig. 21. Layers at the foot coupling.

(Each layer has a compliance that affects comfort and exoskeleton control. The top arrow is body weight loading. The middle arrow is the load applied by the exoskeleton.)

\section{Waist interface}

An off-the-shelf, commercial orthosis will be used to couple the exoskeleton at the waist. Figure 22 shows the device selected, the Model 387x pelvic band component from the Orthomerica Newport 4 Hip Orthosis (www.orthomerica.com). The band has a hard polyethylene outer shell with threaded inserts for mounting components and is lined with soft padding. The band comes in three sizes and the shape of the shell conforms closely to the iliac crest which results in a close, comfortable fit when the component is closed. 


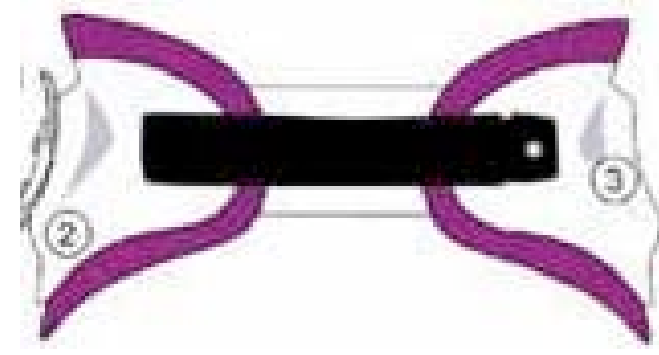

Fig. 22. Waist connection.

(Pelvic band (Model 387x) from the Orthomerica Newport 4 hip orthosis.)

\section{Shoulder Interface}

The coupling at the shoulder will use off-the-shelf, high-performance backpack components (Fig. 23). These provide relatively close coupling to the torso. If tighter coupling is needed for control stability, an off-the-shelf trunk orthosis as shown in Fig. 23 will be used.

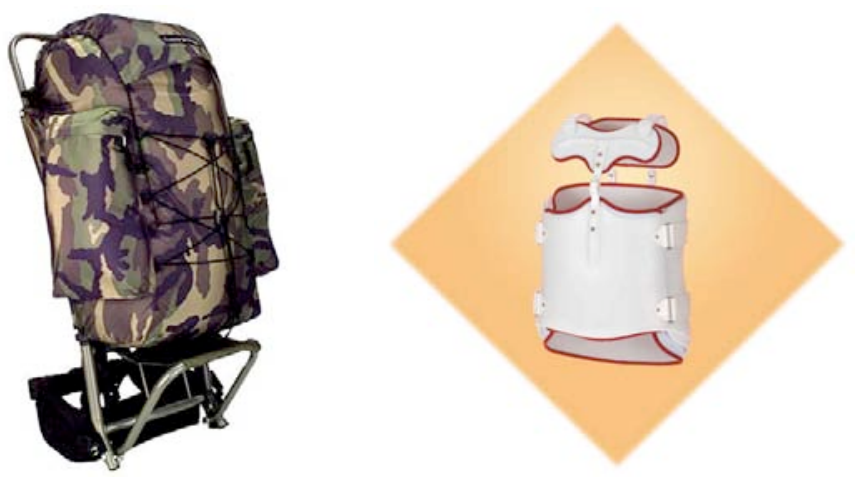

Fig. 23. Shoulder coupling.

(Standard pack components will be used. If tighter coupling is needed, an off-the-shelf trunk orthosis will be used.)

Some of the exoskeleton load will appear like a backpack load to the user. From published studies of backpack loading, gait kinematics, and energy use are impacted by pack load with more perturbations for higher load. There are no hard limits on minimum or maximum loads, which means the amount of loading that the exoskeleton applies to the human need not be tightly specified. Published backpack experiments typically used loads ranging from 10 to $30 \mathrm{~kg}$, although one study looked at loads up to $60 \mathrm{~kg}$ (Legg, 1992). The distribution of the load is important, and for good balance the load should be relatively constant.

\section{Materials}

In addition to standard metal and polymer engineering materials, body attachments will take advantage of the wide range of materials available through the orthosis industry for fitting custom orthotics. Figure 24 shows some of the most useful materials we will use for padding and structural components. 


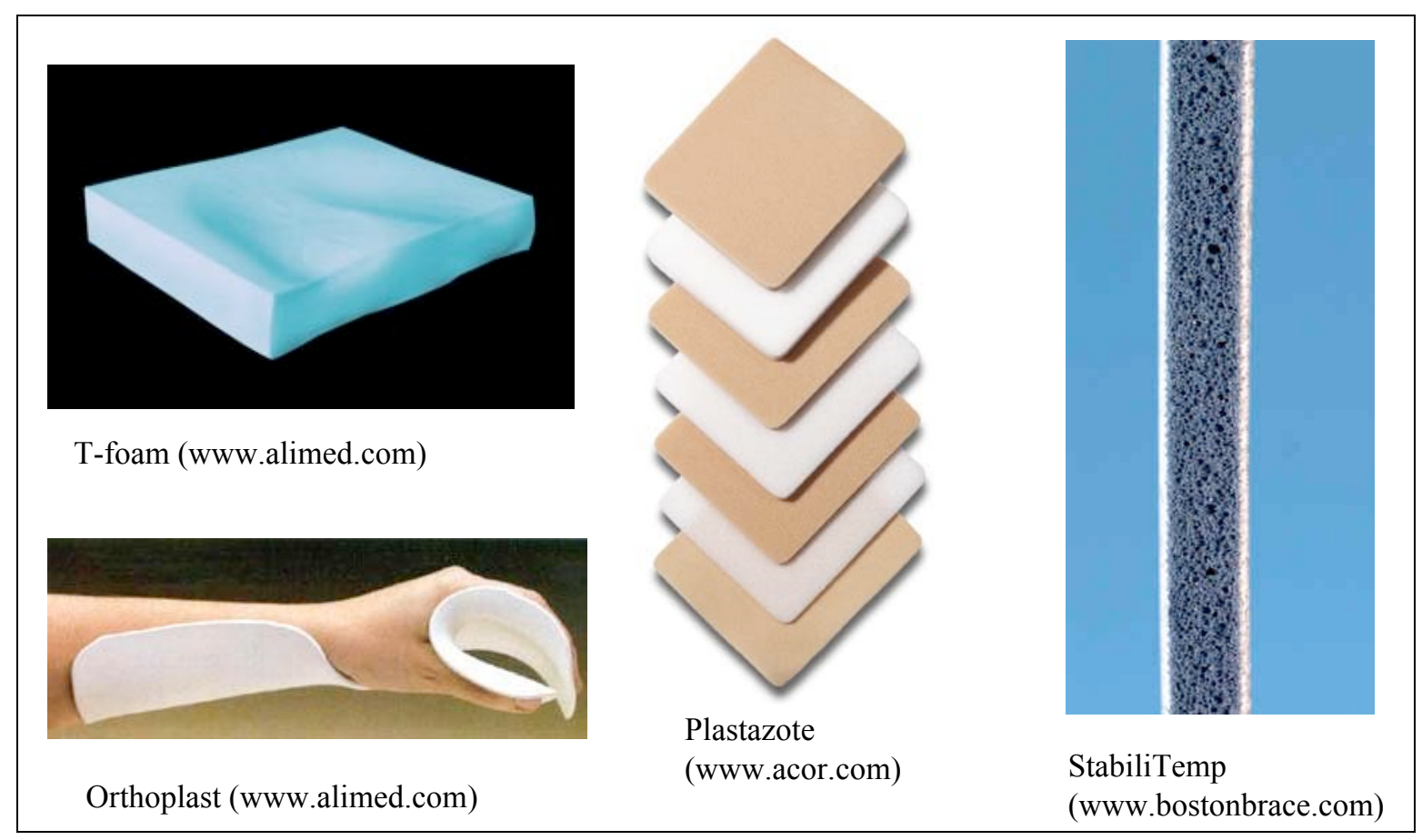

Fig. 24. Common orthotics industry materials.

Polypropylene is the most common sheet material used for rigid cuff and shell type orthotics. Polypropylene can be saw cut, drilled, milled or punched. Drape or vacuum forming is achieved at temperatures of $400^{\circ} \mathrm{F}$. Minor modifications are possible with a hot air gun. Boston Brace (www.bostonbrace.com) provides two grades of polypropylene depending on required stiffness and impact resistance. Sheets come in $1 / 8$ " to $1 / 4$ " thickness.

Low density polyethylene (www.bostonbrace.com) is another common sheet material used in orthotics. Applications are when flexibility and limited strength are needed. Polyethylene can be heat formed at $270^{\circ} \mathrm{F}$.

Orthoplast (www.alimed.com) is a low-heat formed, rigid material that is commonly used to fabricate custom splints and braces. Forming is done after soaking the material in very hot water. Orthoplast is extremely easy to work with.

Thermofoam (www.bostonbrace.com) and Plastizote (www.acor.com) are lightweight, nonallergenic, closed cell polyethylene cushion foams for direct skin contact. The foam comes in $1 / 16$ " to $1 / 2$ " thickness and is ideal for padding because it has good load dissipation in all directions. The material can be cut with a knife or scissors, die cut, or punched. It can be formed using heat and pressure and bonded with a variety of adhesives. The foam is used for liners, pads, splints, and fillers and is the material of choice to line shells and cuffs in upper and lower extremity orthosis.

Durofoam (www.bostonbrace.com) is an open cell polyethylene that maintains approximately constant thickness under compressive loads for long periods of time. It is used where load 
dissipation is needed along with firm support, for example to form pads under a diabetic's foot. It can be bonded to softer foams for more cushioning effect.

Temper-foam (www.alimed.com) is a visco-elastic, conforming material that is commonly used in seating applications. It slowly forms to whatever shape is applied, but is rigid under impact loads.

StabiliTemp (www.bostonbrace.com) is a 3-ply liner material that forms a cool, dry interface by wicking away moisture from the skin into an absorbent gel.

\section{Other Design Considerations}

The coupling of the exoskeleton to the soldier was designed with a quick release feature. For the short-term product development, a quick release coupling simplifies the task of testing exoskeleton prototypes with human subjects. In the long term, a quick release is required should the soldier need to abandon the exoskeleton during emergency situations. For early exoskeleton prototypes, we planned to take advantage of existing quick release products such as the step-in fitting used in high-performance bicycling. 


\section{BIOMECHANICS ANALYSIS}

\section{POWER AND TORQUE REQUIREMENTS ${ }^{\dagger \dagger}$}

A study was performed by ARL to provide baseline estimates of the power and torque requirements for the lower limbs of an exoskeleton for two hypothetical dismounted missions. The missions are "movement to contact" and "clear a building." Human biomedical data reported in journal articles and technical reports were used in this study. The purpose of this work was to provide guidance for the design of the lower limbs of an exoskeletal device. In providing design guidance, there were two goals. The first goal was to provide estimates of the angles, torques, and powers for the ankles, knees, and hips of an exoskeleton based on data collected from humans. The second goal was to calculate the mean power required for various tasks and the total peak power needed by the lower limbs of the exoskeletal device for two "typical" infantry missions.

In order to apply human biomechanical data to design guidance for an exoskeleton, six assumptions were made:

1) The size, mass, and inertial properties of the exoskeleton will be equivalent to those of a human.

2) The exoskeleton will carry itself (including power supply) and the soldiers' load.

3) The joint torques and joint powers scale linearly with mass.

4) The exoskeleton's gait will be the same as a human's gait.

5) The exoskeleton will carry a load on its back in the same way that humans carry loads on their backs.

6) The exoskeleton will move at the same speed, cover the same distance, and carry the same load as a soldier who does not have an exoskeleton.

The human biomechanical data used in this analysis came from studies reported in relevant journals and technical reports. These data are from studies of normal walking, walking at various speeds, walking while loads are carried, running at a moderate pace, and running at various speeds. Other activities for which joint angle, torque, and power data were obtained included stair climbing, jumping, and kneeling. The figures in Appendix $\mathrm{C}$ show the joint angles, joint moments, and joint powers for walking, walking while a load is carried, running, ascending stairs, descending stairs, and jumping. It is important to note that the joint power data are derived from calculations of the mechanical work done by the lower limbs to move the entire body, as opposed to physiological work based on oxygen consumption.

The calculation of total peak power focused on two hypothetical missions, a movement-tocontact mission and a clear-building mission. These missions represent the kind of diverse missions for which an exoskeleton might be used. Also, the fundamental tasks (e.g., walking, jogging, etc.) involved in these missions are the same tasks that occur in other infantry missions (e.g., infiltrate and raid). The movement-to-contact mission took place over a 16-hour period, and the exoskeleton carried the soldier's sustainment load $(35 \mathrm{~kg})$ during most of that time. In the clear-building mission, which lasted approximately 2 hours, the exoskeleton carried the

\footnotetext{
"† This section summarizes the ARL study documented in Crowell et al., 2002.
} 
soldier's fighting load $(24 \mathrm{~kg})$. The soldier in these hypothetical missions was a $50^{\text {th }}$ percentile male whose mass was $77 \mathrm{~kg}$.

The data used to calculate total peak power for the movement-to-contact and clear-building missions came from the joint power data in Appendix C. Five simplifying assumptions about gait and the exoskeleton were made in order to calculate peak power. The assumptions were:

1) Increasing the load carried has the same effect on peak power as does increasing body mass.

2) Joint powers in the frontal and transverse planes are small compared to the sagittal plane. Therefore, only sagittal plane peak power profiles were calculated.

3) Normal gait is symmetrical.

4) Peak power values for assuming and leaving kneeling and prone positions, crawling, and climbing a ladder can be approximated with the values for stair ascent.

5) Power values of other lower extremity joints, such as the metatarsophalangeal joint, are small and therefore not included in the peak power calculation.

Then, a four-step process was used to calculate the peak power required by the lower limbs of the exoskeleton during the movement-to-contact and clear-building missions.

1) Spreadsheets that listed each task in chronological order (e.g., walking, jogging, jumping, etc.) that occurred during the missions were created. The time required for each task and the load type are listed in Table 1.

2) The powers required at each joint (left and right ankle, knee, and hip) were summed, and peak and average power values were identified for each task and load combination (Fig. 25 and Table 2).

3) The peak power for each task and load combination was entered into the spreadsheet.

4) The peak power and the time required for each task were plotted for each mission (Figs. 26 and 27).
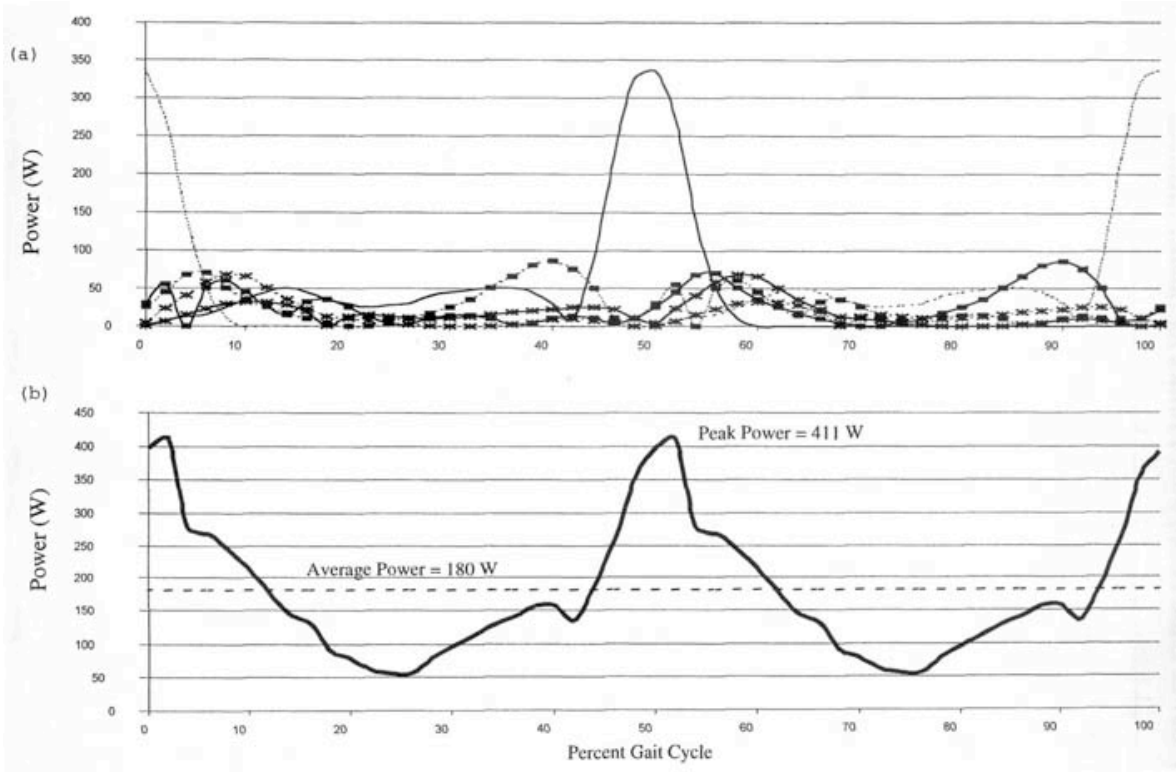

Fig. 25. Peak and average power.

(Example of one cycle of a task and load combination for all six lower limb joints.) 
Table 1. Sample time-line of a typical soldier mission

\begin{tabular}{ccc}
\hline Time (min) & Task & Load \\
\hline 0 & Walking & Sustainment \\
45 & Resting & \\
60 & Walking & Sustainment \\
90 & Kneeling and Resting & Sustainment \\
92 & Walking & Sustainment \\
110 & Resting & \\
120 & Walking & Sustainment \\
130 & Kneeling and Resting & Sustainment \\
124 & Walking & Sustainment \\
150 & Prone - Security & Sustainment \\
152 & Walking & Sustainment \\
170 & Resting & \\
180 & Walking & Sustainment \\
200 & Prone - Security & Sustainment \\
205 & Walking & Sustainment \\
230 & Resting & \\
240 & Walking & Sustainment \\
\hline
\end{tabular}

Table 2. Peak and average power values for typical soldier tasks

\begin{tabular}{lcc}
\hline & \multicolumn{2}{c}{ Total mass (soldier plus carried load) } \\
Task & Fighting $(101 \mathrm{~kg})$ & Sustainment $(112 \mathrm{~kg})$ \\
\hline Slow walk (86 steps/min) & $283(119)$ & $314(131)$ \\
Natural walk (105 steps/min) & $411(180)$ & $456(200)$ \\
Fast walk (122 steps/min) & $693(315)$ & $769(349)$ \\
Jog $(2.7 \mathrm{~m} / \mathrm{s})$ & $1966(1041)$ & $2180(1155)$ \\
Run & $3147(1693)$ & $3489(1877)$ \\
Sprint $(7.6 \mathrm{~m} / \mathrm{s})$ & $5547(3522)$ & $6151(3906)$ \\
Running vertical jump & $3559(1325)$ & $3947(1369)$ \\
Ascend stairs & $501(229)$ & $556(254)$ \\
Descend stairs & $478(270)$ & $530(300)$
\end{tabular}

Note: All peak (average) power values are given in watts. All values represent absolute power for all six lower extremity joints (left and right hip, knee, and ankle joints) during the given task and load combination, except for jumping, which assumes a running jump with single-legged take-off. 


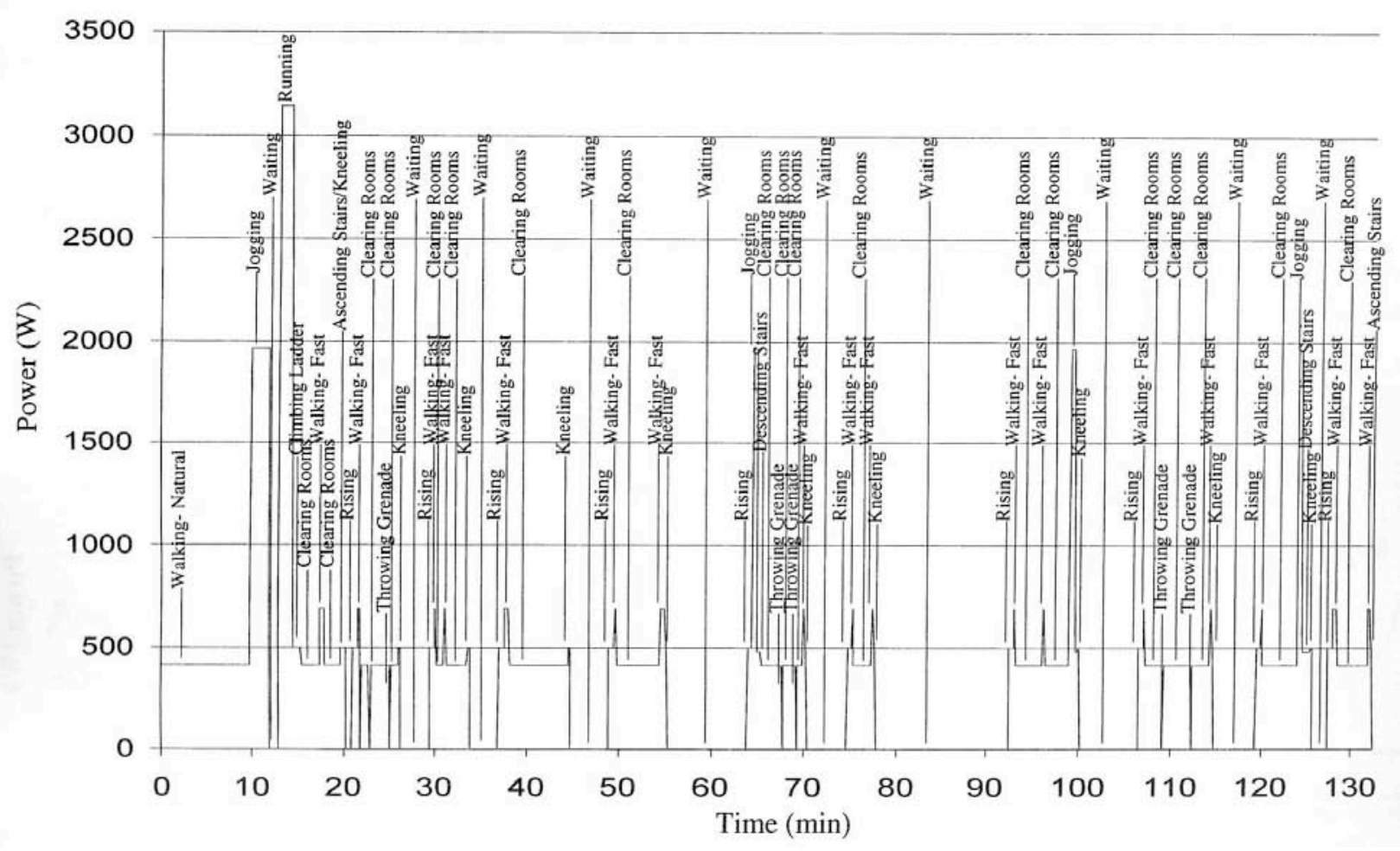

Fig. 26. Lower limb peak power profile for clear-building mission scenario.

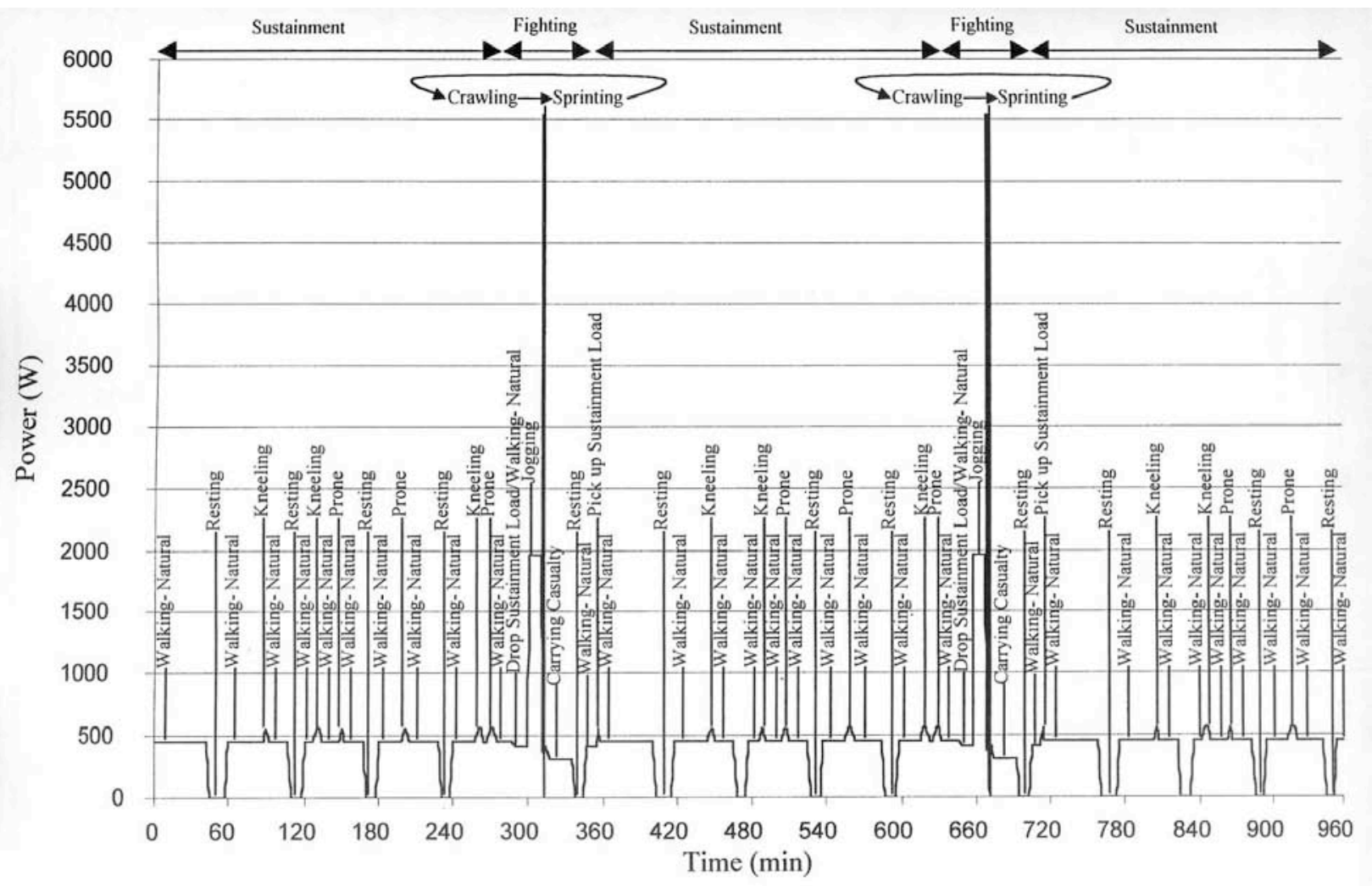

Fig. 27. Lower limb peak power profile for movement-to-contact mission scenario. 
The accuracy of the calculated results is in part a reflection of the accuracy of the original data. When biomechanical data are collected, certain assumptions, limitations, and variabilities affect the accuracy of the data. The accuracy of data collected in biomechanical studies is generally within $20 \%$ of their real values. It is likely that when these data are used to calculate kinetic variables such as joint moments and powers, compensating errors keep the overall accuracy of the results within $20 \%$ of their true values. Therefore, the power requirements calculated in this report have a tolerance of $\pm 20 \%$.

During most of the movement-to-contact mission, the soldier and exoskeleton walked at a natural pace, and the exoskeleton carried the sustainment load. Although the average power required for this task was $200 \mathrm{~W} \pm 40 \mathrm{~W}$, there were peak power requirements of $500 \mathrm{~W} \pm 100 \mathrm{~W}$. The peak powers are important because the power supply must be sized to meet the peak requirements. If the power supply cannot meet the peak requirements at the proper frequency, then the exoskeleton may, in contrast to its goal, decrease the soldier's speed, strength, and endurance. The $500 \mathrm{~W} \pm 100 \mathrm{~W}$ peaks occurred at approximately $2 \mathrm{~Hz}$. This frequency coincides with ankle plantarflexion that occurs during "toe-off" in each gait cycle. When contact was made with the enemy, part of the sustainment load was dropped, and the soldier and the exoskeleton performed a variety of tasks including sprinting. The average power required during this part of the mission was $3500 \mathrm{~W} \pm 700 \mathrm{~W}$ with peaks of $5500 \mathrm{~W} \pm 1100 \mathrm{~W}$ that occurred at $4 \mathrm{~Hz}$. The $4-\mathrm{Hz}$ frequency coincides with ankle plantarflexion during toe-off for sprinting.

The power requirements for the clear-building mission were more variable than the power requirements for the movement-to-contact mission. This occurred because the soldier changed his speed many times as he moved to the building and then from room to room. For the clearbuilding mission, the average power required was $200 \mathrm{~W} \pm 40 \mathrm{~W}$ to $300 \mathrm{~W} \pm 60 \mathrm{~W}$ during much of the mission. Peak powers rose to $400 \mathrm{~W} \pm 80 \mathrm{~W}$ for walking at a natural pace and frequently to $700 \mathrm{~W} \pm 140 \mathrm{~W}$ for walking at a fast pace. There were several times when the soldier was jogging. At those times, the average power was $1000 \mathrm{~W} \pm 200 \mathrm{~W}$, and peak power as $2000 \mathrm{~W}$ $\pm 400 \mathrm{~W}$. The highest peak power requirement for this mission (approximately $3000 \mathrm{~W} \pm 600 \mathrm{~W}$ ) occurred at the beginning when the soldier was running up to the building. As with the movement-to-contact mission, the peak powers must be delivered at approximately 2 to $4 \mathrm{~Hz}$.

In conclusion, the data provided through this analysis can be used as a baseline for the initial design of an exoskeletal device. These data can be used to evaluate currently available and nearterm technology to determine the feasibility of developing a practical exoskeleton. The joint angles, torques, and powers presented in Appendix $\mathrm{C}$ and Table 2 provide a basis for initial design of lower limb joints and actuators, and the peak power profiles (Figs. 25 and 26) provide a basis for initial sizing of the power supply. 


\section{HUMAN FACTORS ANALYSIS}

\section{SOLE THICKNESS OF THE BOOT INTERFACE ${ }^{\ddagger \ddagger}$}

The exoskeleton boot interface, an integral component of the system, is expected to incorporate a 1- to 2-inch thick sole for the containment of force sensors, thus creating a substantial offset of the soldier's foot from the ground. Very little published data exists regarding human factors issues related to footwear sole thickness, therefore, the objective of this investigation was to identify, through analyses of biomechanics data, observations of ability to negotiate obstacles and subjective assessments, potential human factors issues related to the additional sole thickness of an exoskeleton boot interface. The results of this investigation will provide critical guidance to the DARPA EHPA Program for the development of an exoskeleton boot interface that is safe, comfortable, and functional, and may also result in substantial savings of time and money for the EHPA Program.

In this investigation, three-dimensional angles and sagittal plane moments and powers at the right hip, knee, and ankle joints during walking, running, squatting, and right and left kneeling; stride length, velocity, cadence, and percent stance during walking and running; cycle time during squatting and right and left kneeling; time to negotiate obstacles; and ratings of comfort, stability and difficulty during walking, running, squatting, right and left kneeling, and negotiating obstacles were evaluated for three sole thicknesses-baseline (no additional sole), 1-inch and 2-inch. Rubber overboots with detachable wooden soles were used to create the three-sole conditions (Fig. 28). For each of the three-sole conditions, three-dimensional positions of reflective markers placed on the participants' boots and at several anatomical locations (Fig. 29), were tracked by six high-resolution video cameras connected to a computer running EVaRT 4.0 motion capture software (Motion Analysis Corporation, Santa Rosa, California), as participants walked, ran, squatted, kneeled on their right knee, and kneeled on their left knee (Fig. 30) on a force plate (Advanced Mechanical Technology, Inc., Newton, Massachusetts) surrounded by a raised walkway. Biomechanics variables were calculated from ground reaction force and three-dimensional marker position data using KinTrak 6.2 biomechanical analysis software (Motion Analysis Corporation, Santa Rosa, California). Individual completion times under the three-sole conditions were collected for five obstacles on the Army Research Laboratory (ARL) Mobility-Portability course (Aberdeen Proving Ground, Maryland) - log balance, elevated up and down, tires, house, and zig-zag (Fig. 31). Subjective assessments of comfort, stability and difficulty during walking, running, squatting, right and left kneeling, and negotiating the five obstacles under the three-sole conditions were obtained using a questionnaire with a six-point rating scale (very low, low, slightly low, slightly high, high, very high).

Twelve male soldiers (age: $21 \pm 3 \mathrm{yr}$, height: $172.5 \pm 8.6 \mathrm{~cm}$, body mass: $76 \pm 9 \mathrm{~kg}$ ), stationed at Aberdeen Proving Ground, were recruited for participation in this study. All participants were first briefed on the objectives of this investigation and procedures to be followed, and then asked to sign a statement of informed consent. After signing the volunteer agreement, height, weight, and shoe size were measured and recorded for each participant. Prior to data collection for each

\footnotetext{
This section summarizes the ARL study documented in Boynton and Crowell, 2003 that is currently in publishing.
} 
sole condition, participants were trained to walk, run, squat, and kneel on the force plate and to negotiate each of the five obstacles correctly, and then permitted to practice until they were able to perform each activity comfortably.
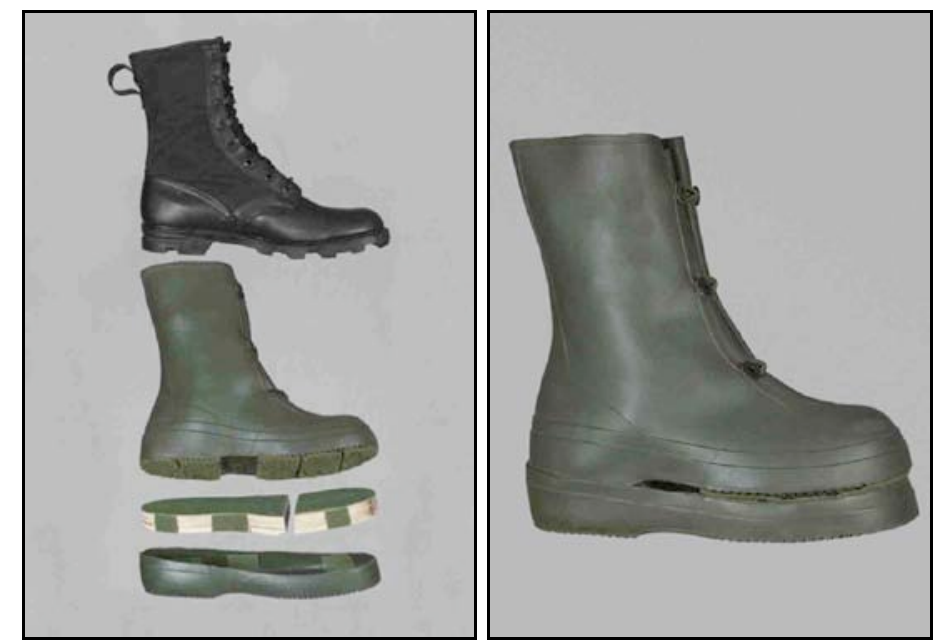

Fig. 28. Overboot and sole set-up.
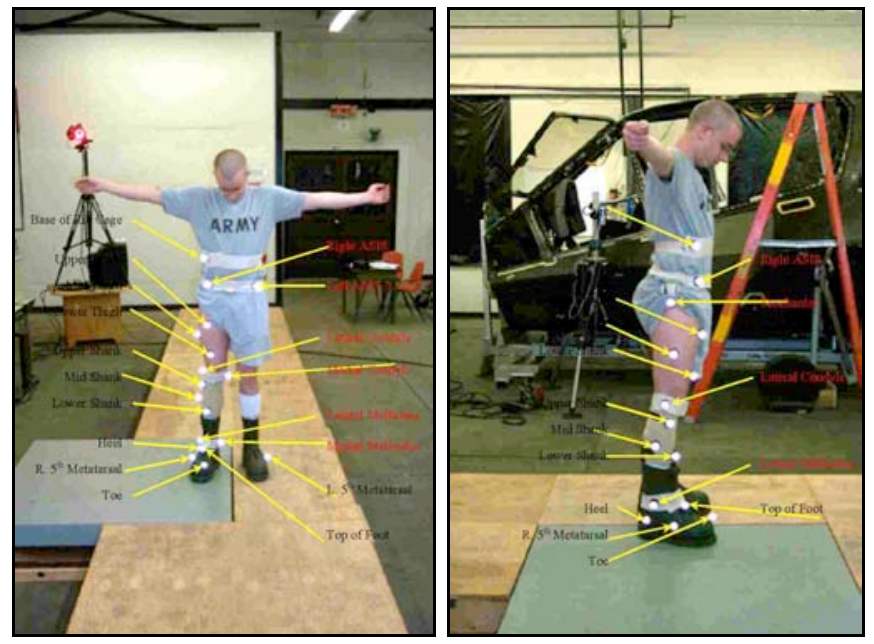

Fig. 29. Reflective marker placement.

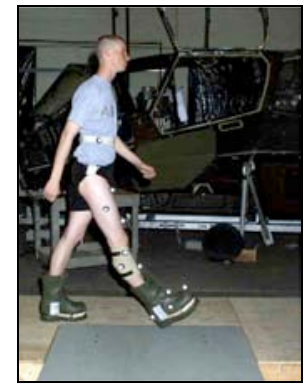

Walking

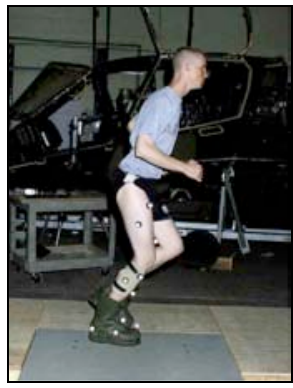

Running

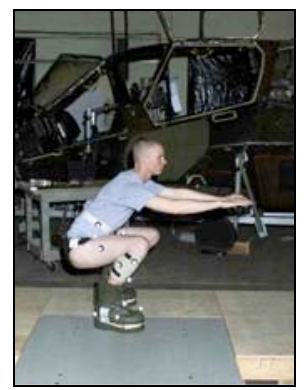

Squatting

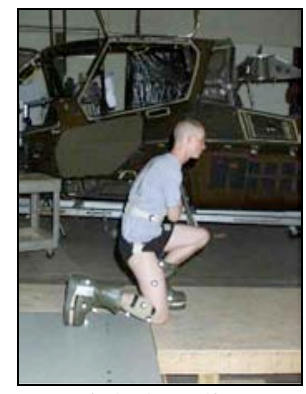

Right kneeling

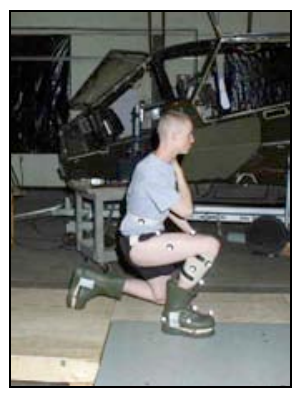

Left kneeling

Fig. 30. Activity on the walkway and force plate. 


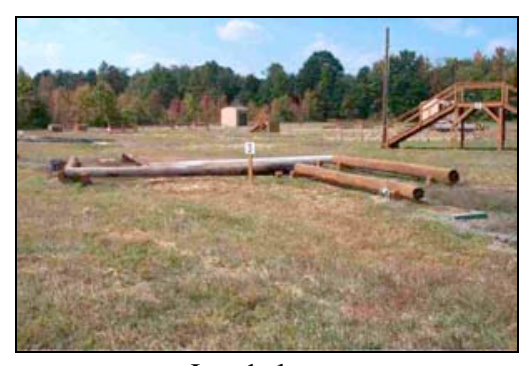

Log balance

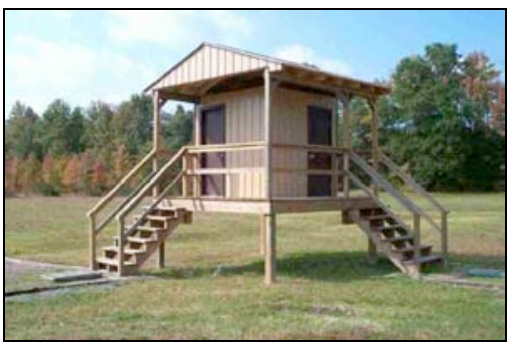

House

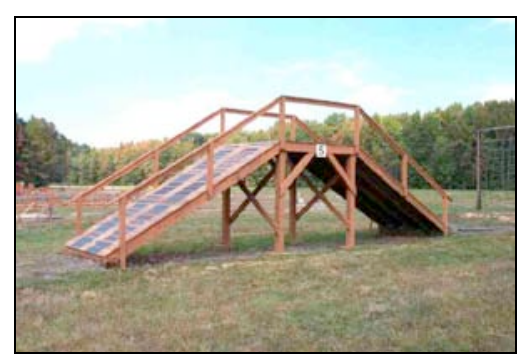

Elevated up and down

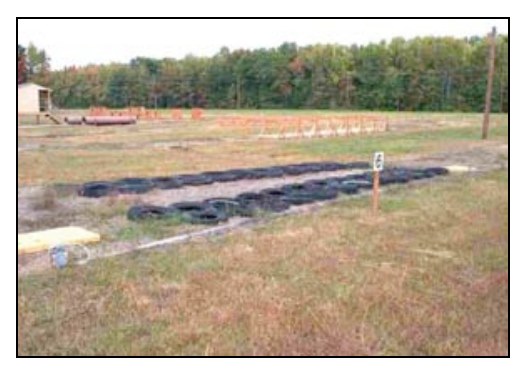

Tires

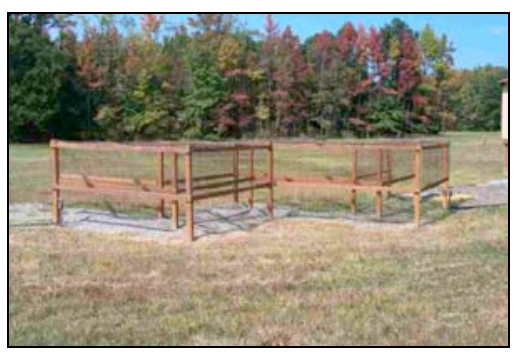

Zig-zag

Fig. 31. Obstacles on the ARL mobility-portability course.

A within-subjects design with a Williams Square counterbalancing scheme for sole condition was used in this investigation. The independent variable was sole thickness, which consisted of three levels: baseline, 1-inch and 2-inch. The dependent variables were:
a. peak three-dimensional joint angles at the hip, knee, and ankle (degrees),
b. peak sagittal plane joint moments at the hip, knee, and ankle $(\mathrm{N}-\mathrm{m} / \mathrm{kg})$,
c. peak sagittal plane joint powers at the hip, knee, and ankle (W/kg),
d. peak ground reaction forces $(\mathrm{N} / \mathrm{BW})$,
e. stride length (m),
f. velocity $(\mathrm{m} / \mathrm{s})$,
g. cadence (steps/min),
h. percent stance,
i. cycle time ( $\mathrm{sec})$,
j. obstacle completion time (sec), and
k. ratings of comfort, stability and difficulty.

A univariate analysis of variance (ANOVA) with main effects for participant, order, and sole conditions was performed on each dependent variable. Those variables significantly affected by sole condition were further analyzed using a Fisher's Least Significant Difference (LSD) posthoc test in order to identify specific differences between means. ANOVA and Fisher's LSD results were considered significant for values less than 0.05. All statistical analyses were performed using SPSS 10.1 for Windows (SPSS, Inc., Chicago, Illinois). 
Summaries of the biomechanics data for walking, running, squatting, and right and left kneeling under each of the three-sole conditions can be found in Tables 7-11 in Appendix D, respectively. Stride length, velocity, cadence, percent stance, and cycle time are reported as mean values. Angles, ground reaction forces, moments, and powers are reported as means of the peak values.

During walking, twelve biomechanics variables were found to be significantly affected by sole condition- peak hip power generation; peak knee flexion, external rotation, power generation and absorption; peak ankle dorsiflexion, abduction, internal rotation, plantarflexor moment and dorsiflexor moment; peak vertical ground reaction force; and peak propulsive ground reaction force (Table 7, Appendix D). Compared to the baseline sole condition, 1-inch and 2-inch sole condition mean values were found to be significantly smaller for ankle abduction (39.6 and $53.0 \%$ ) and internal rotation (25.6 and 35.6\%); knee power generation (22.2 and 34.1\%) and absorption (14.1 and 44.1\%); and propulsive ground reaction force (8.0 and 16.0\%); and significantly larger for ankle dorsiflexion (20.8 and 24.2\%), plantarflexor moment (11.8 and 9.7\%) and dorsiflexor moment (61.9 and 52.4\%). Mean values under the 2-inch sole condition were shown to be significantly smaller than both baseline and 1-inch sole condition means for knee flexion (6.0 and 4.5\%) and power absorption (44.1 and 34.9\%); hip power generation (20.4 and $17.1 \%)$, and propulsive ground reaction force (16.0 and 8.7\%). Additionally, compared with the baseline sole condition, knee external rotation under the 2-inch sole condition was found to be significantly reduced by 14.7 percent.

Only one running biomechanics variable, peak ankle abduction, was found to be significantly affected by sole condition (Table 8, Appendix D). Post-hoc test results further revealed that the 1-inch and 2-inch sole condition mean values were significantly reduced below that of the baseline sole condition by 37.3 and 35.8 percent, respectively.

Sole condition was not found to have a significant effect on any of the biomechanics variables for squatting (Table 9, Appendix D).

A significant effect of sole condition was found for nine right kneeling biomechanics variablescycle time; peak hip flexion; peak knee flexion (maximum) and external rotation; peak ankle abduction, external rotation, internal rotation and power generation; and peak vertical ground reaction force (Table 10, Appendix D). Mean 1-inch and 2-inch sole condition values were found to be significantly larger than mean baseline values for cycle time (10.8 and 19.0\%), hip flexion (59.2 and 76.7\%), knee flexion (10.2 and 8.3\%), and ankle power generation (73.0 and $113.5 \%$ ), and significantly smaller than mean baseline values for ankle abduction (63.8 and $87.8 \%$ ) and internal rotation (39.3 and 47.9\%). Vertical ground reaction force, knee external rotation and ankle external rotation means under the 2-inch sole condition were also found to be significantly increased over the baseline sole condition by 16.7, 33.8 and 78.3 percent, respectively. Additionally, compared with 1-inch sole condition means, 2 -inch sole condition means were significantly larger for cycle time (7.4\%) and vertical ground reaction force (11.4\%).

Five left kneeling variables were found to be significantly affected by sole condition- peak hip abduction; peak knee flexion (maximum) and external rotation; and peak ankle dorsiflexion (maximum) and external rotation (Table 11, Appendix D). In comparison to baseline sole condition means, 1-inch and 2-inch sole condition means were found to be significantly larger for maximum knee flexion (3.5 and 7.9\%) and knee external rotation (15.7 and 26.9\%). Mean 2inch sole condition means for hip abduction, and ankle dorsiflexion and external rotation were 
also shown to be significantly increased over those of the baseline sole condition, by $21.9,14.4$ and 35 percent, respectively. Additionally, maximum knee flexion under the 2-inch sole condition was found to be $4.3 \%$ larger than that under the 1-inch sole condition.

Mean obstacle completion times under each of the three sole conditions are shown in Table 12 (Appendix D). A statistically significant effect of sole condition on completion time was identified for the Log Balance and Tires only. For the Log Balance, 1-inch and 2-inch sole condition completion times were found to be significantly longer than for the baseline sole condition, by 21.3 and $26.0 \%$, respectively. Additionally, in comparison to the baseline sole condition, the Tires completion time for the 2 -inch sole condition was significantly increased by 11.5 percent.

In order to compute mean values and conduct statistical analysis of the questionnaire responses, each response level was assigned a numerical value: 1=very low, 2=low, 3=slightly low, 4=slightly high, 5=high, 6=very high. Mean responses to the comfort, stability and difficulty questionnaire are given in Fig. 101 (Appendix D). With the exception of Squatting, House and Zig-Zag difficulty, all questionnaire responses were found to be significantly affected by sole condition. As a whole, ratings of comfort and stability were found to decrease, while ratings of difficulty were found to increase, with increasing sole thickness.

Few of the biomechanics variables examined were identified as being significantly affected by sole condition (approximately 14.5\%). It is especially notable that peak moment and power values at all three joints are similar across sole conditions, with the exceptions during walking of hip power generation and knee power generation and absorption, which decrease, and ankle plantarflexor and dorsiflexor moments, which increase, as sole thickness increases. Thus sole thicknesses up to 2 inches should not have a negative impact on the power requirements of the exoskeleton system, and actuators can be designed to provide additional torque at the ankle joints. Although sole thickness was found to have a significant effect on various joint angles during walking, running, and kneeling, it appears that humans are able to accommodate these differences, maintaining similar values across sole thickness conditions for each of the temporalspatial variables examined. The significantly longer completion times observed for the Log Balance and the Tires are most likely the result of decreased stability as sole thickness increased. Although sole condition was shown to have a significant effect on perceptions of comfort, stability and difficulty, it should be noted that on average the comfort rating for the 2-inch sole condition was slightly high while that for the baseline condition was high, and the difficulty rating for the 2-inch sole condition was slightly low while that for the baseline was low. The largest disparity was seen for stability with the average 2-inch sole condition rating being slightly low as compared to an average rating of high for the baseline condition. It may be possible to eliminate differences between the baseline and the 1-inch or 2-inch conditions through training or by designing the components of the exoskeleton so that they promote comfort and stability. Overall, it appears that the exoskeleton boot interface could incorporate a sole up to 2 inches in thickness without considerable impact on the human factors issues investigated in this study. 


\section{BIOMECHANICS TESTING}

\section{CONTROLLER LAG EFFECTS ON AN OPERATOR WALKING}

The exoskeleton force-reflecting controller is maximally challenged at heel strike and toe off when rapid dynamics occur. In the event that the controller does not keep up with the human during certain maneuvers when the foot is coming up off the ground, the user will feel a sensation not unlike wearing a pair of sticky boots. That is, the controller may exert brief forces on the foot with the force vector directed towards the ground. This is the same effect that many haptic interfaces have when emulating stiff walls. When the user taps the virtual wall with the haptic interface, he or she may feel a slight "stick" as the controller attempts to emulate the high bandwidth mechanical events that occur during rigid object collisions.

To explore what impact these control delays would have upon gait, and to determine whether padding was required under the tongue of the boot, a simple pilot experiment was conducted. The purpose of the experiment was to see what it was like to walk in a "sticky" boot. Although one could design an exoskeleton with very high bandwidth actuators and control, if the stickiness is tolerable, more cost-effective, lower bandwidth components would be adequate.

A single "sticky" boot was constructed by fastening disk magnets to the underside of a standard hiking boot. The boot was a Cherokee, size 12, right hiking boot purchased from a discount store. The magnets were ceramic pot magnets, 1.4-in dia., 0.281-in thick with a maximum 13-lb pull (McMaster Carr 5685 K26). Magnets were fastened to the sole of the boot with 10-24 x 1/2" machine screws. An 1/8" brad point drill bit was used to make pilot holes in the sole. The screws were self-threaded into the sole, a fastening means that was sufficiently secure for brief experiments. Eight magnets were used, four fastened to the heel and four to the sole. Two sheets of 12" x 24", 22 g plain sheet steel were taped end to end on the floor to provide a target area for the shoe. Photographs of the apparatus appear in Fig. 32.

A single subject walked, sometimes over the target area and sometimes over the adjacent plain floor. The sticky boot was on the right foot. The left foot wore the matching, but unmodified, left boot. The magnet pull when on the steel sheet varied depending upon how many magnets were in contact and whether the magnets were flat against the plate. With all magnets making good contact and the foot pulling straight up, the release load was $35 \mathrm{lb}$. When the foot was rolled up, as is normal during gait, the release load dropped to $1-2 \mathrm{lb}$ because it is relatively easy to release the magnets through rotation.

The results of this unstructured pilot showed that small amounts of force keeping the foot on the ground can be tolerated quite well with only a modest impact on gait. Further, only minimal padding is needed under the laces to distribute the extra loads comfortably. Special user training would be required to accommodate the soldier to these unusual forces should they be an inevitable part of the controller. More rigorous human factors testing of "sticky" boots is needed to determine the true impact on gait. 

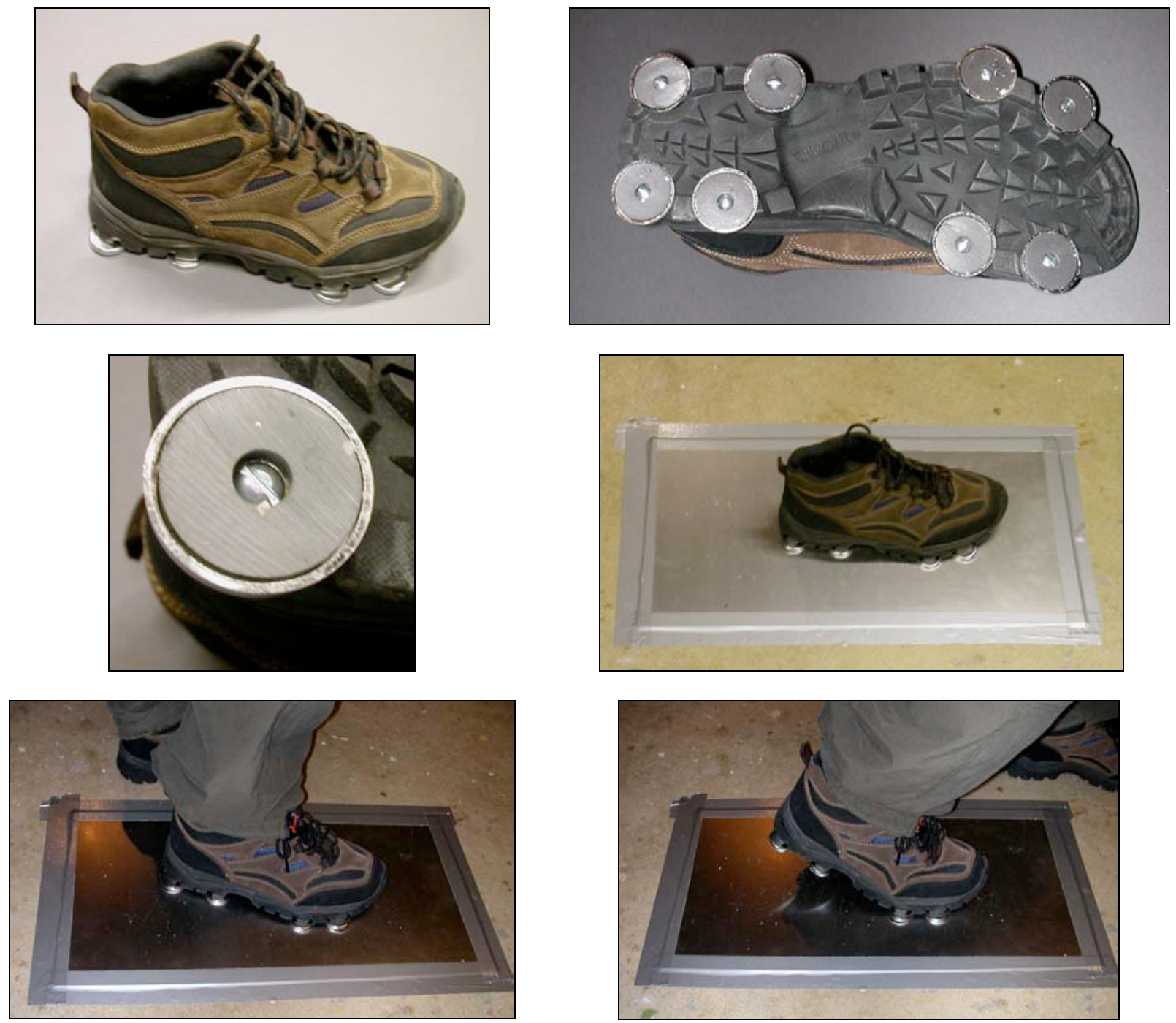

Fig. 32. "Sticky" boot.

(Eight disk magnets attached to sole. Walking over sheet steel with boot.) 


\section{EXOSKELETON MOCKUP}

To determine whether the selected exoskeleton joints and degrees of freedom would interfere or complement normal gait or mobility maneuvers, a simple mockup of the exoskeleton structure was fabricated. Fabrication materials included aluminum bar and tube, wood dowels, an off the shelf orthotic pelvic band (Newport 4 hip orthosis, Orthomerica, www.orthomerica.com), and a standard hiking boot. Only one side of the orthosis was mocked up. The intent was to produce a structure that was faithful in kinematics to the powered exoskeleton, but not in actuation or load carrying. Figure 33 shows the mockup during quiet standing.

The mockup had an ankle flexion/extension and foot inversion/eversion joints (Fig. 34), a knee flexion/extension joint, and a thigh prismatic joint (Fig. 35), and hip flexion/extension and internal/external rotation joints (Fig. 36). The hip abduction/adduction joint was not implemented in the mockup.

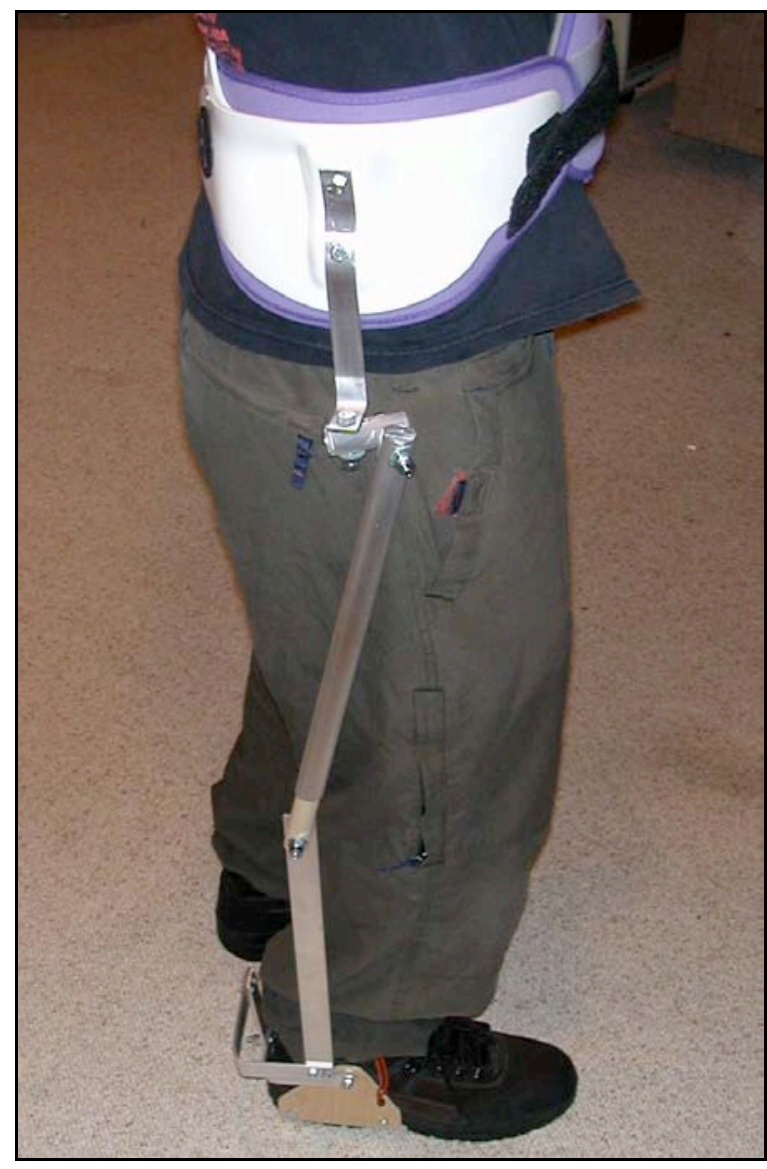

Fig. 33. Exoskeleton mockup.
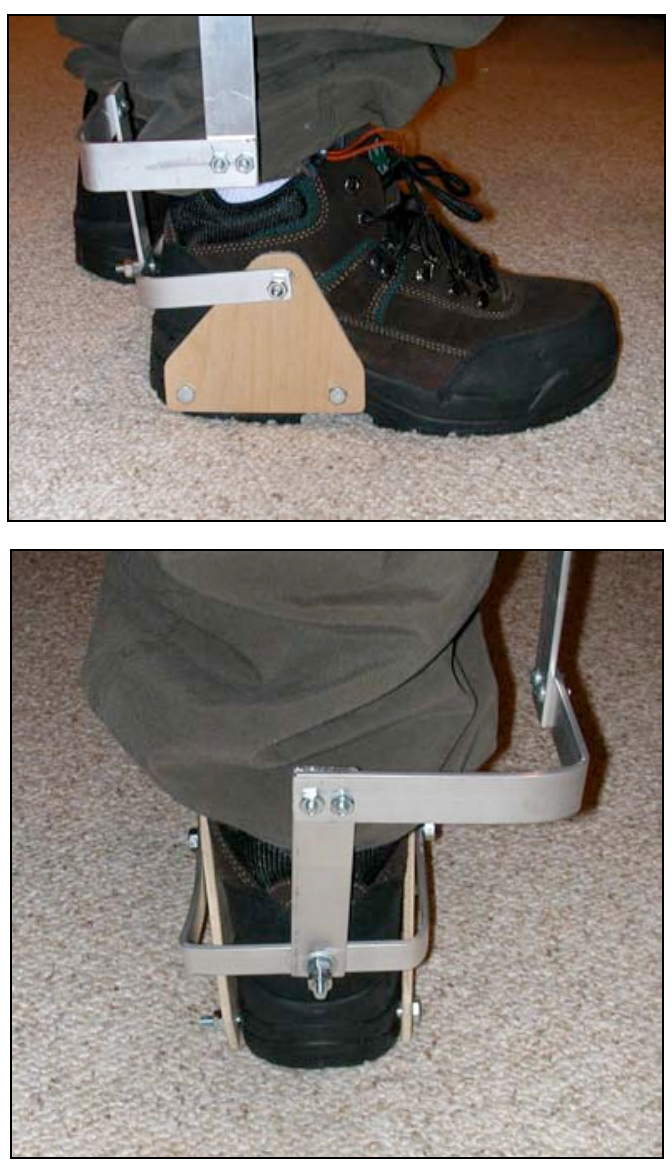

Fig. 34. Ankle flexion/extension and foot inversion/eversion joints. 


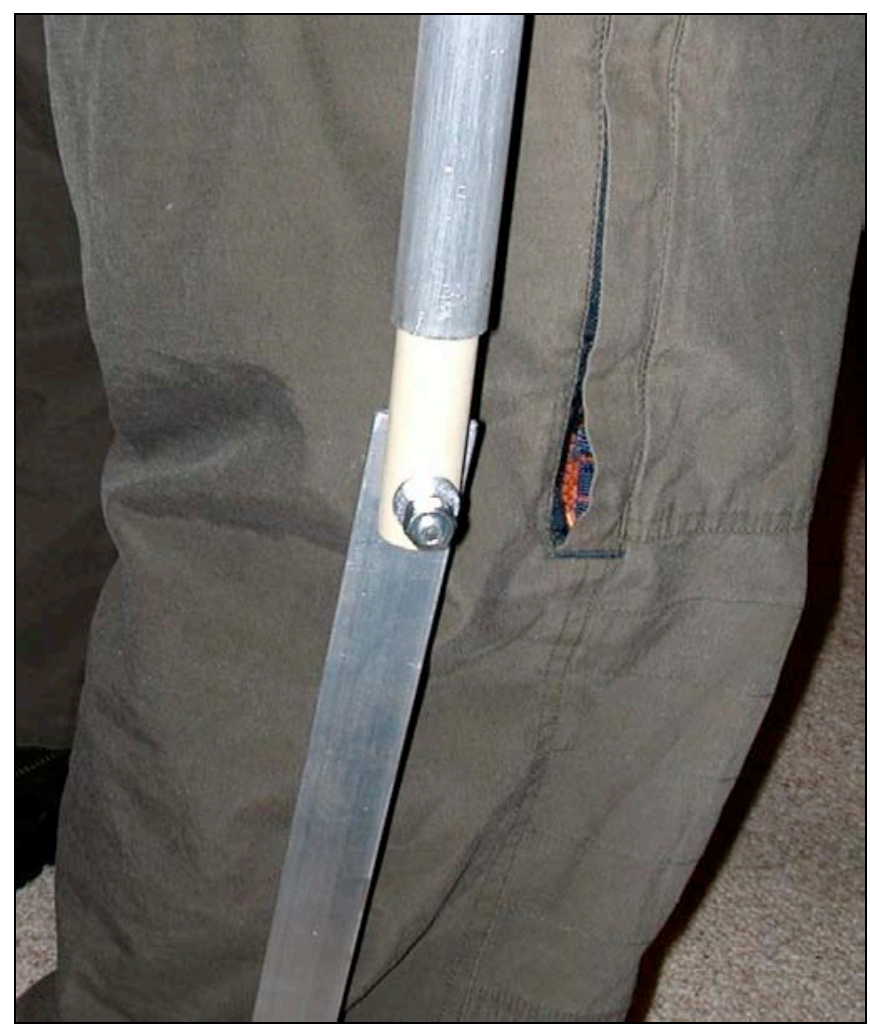

Fig. 35. Knee flexion/extension and thigh prismatic joints.
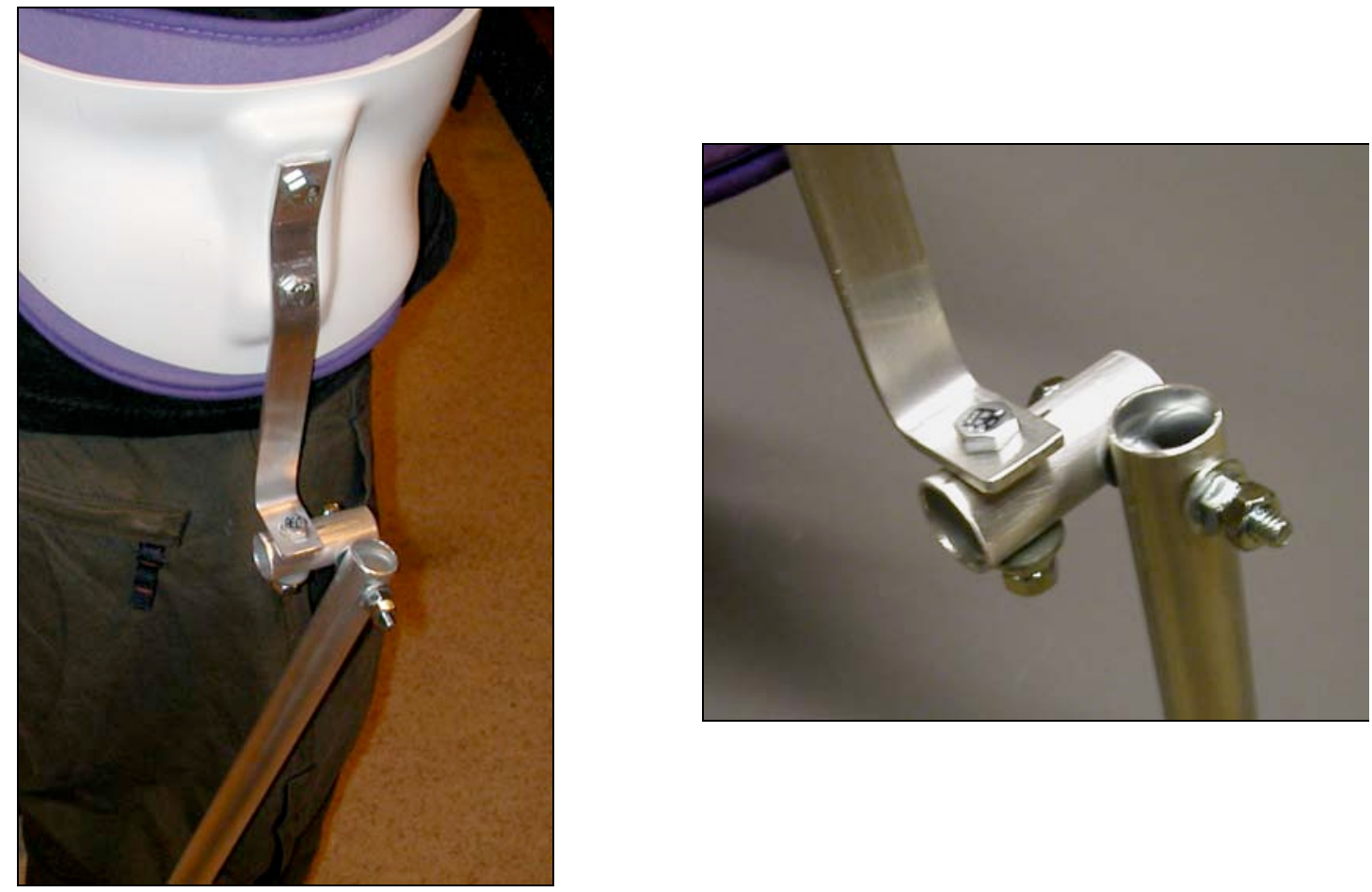

Fig. 36. Hip flexion/extension and internal/external rotation joints. 
The mockup was able to follow the body during normal maneuvers. Figure 37 shows the foot and ankle during toe-off of a gait cycle. Figure 38 demonstrates that the kinematics are able to follow a deep knee bend.

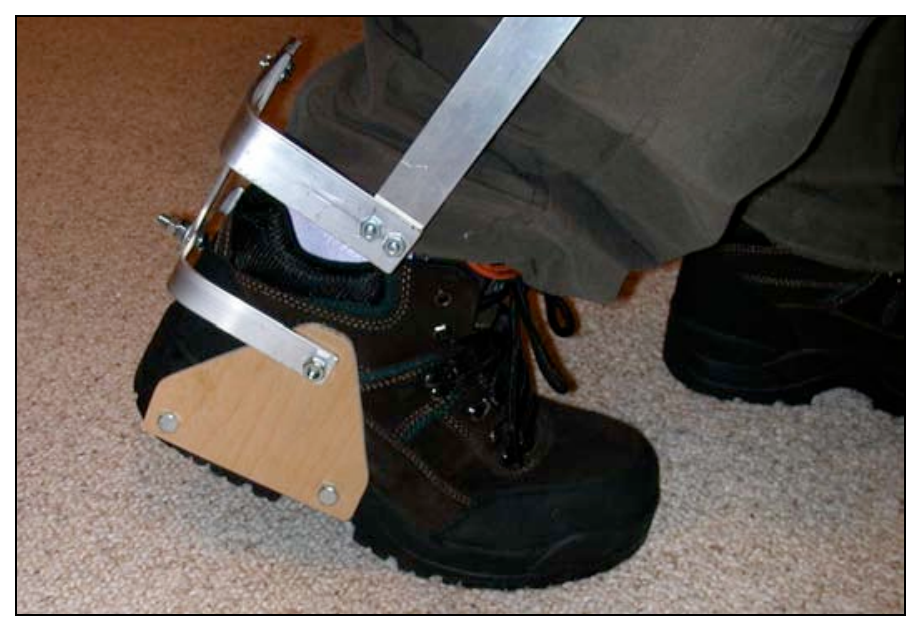

Fig. 37. Ankle during toe-off.

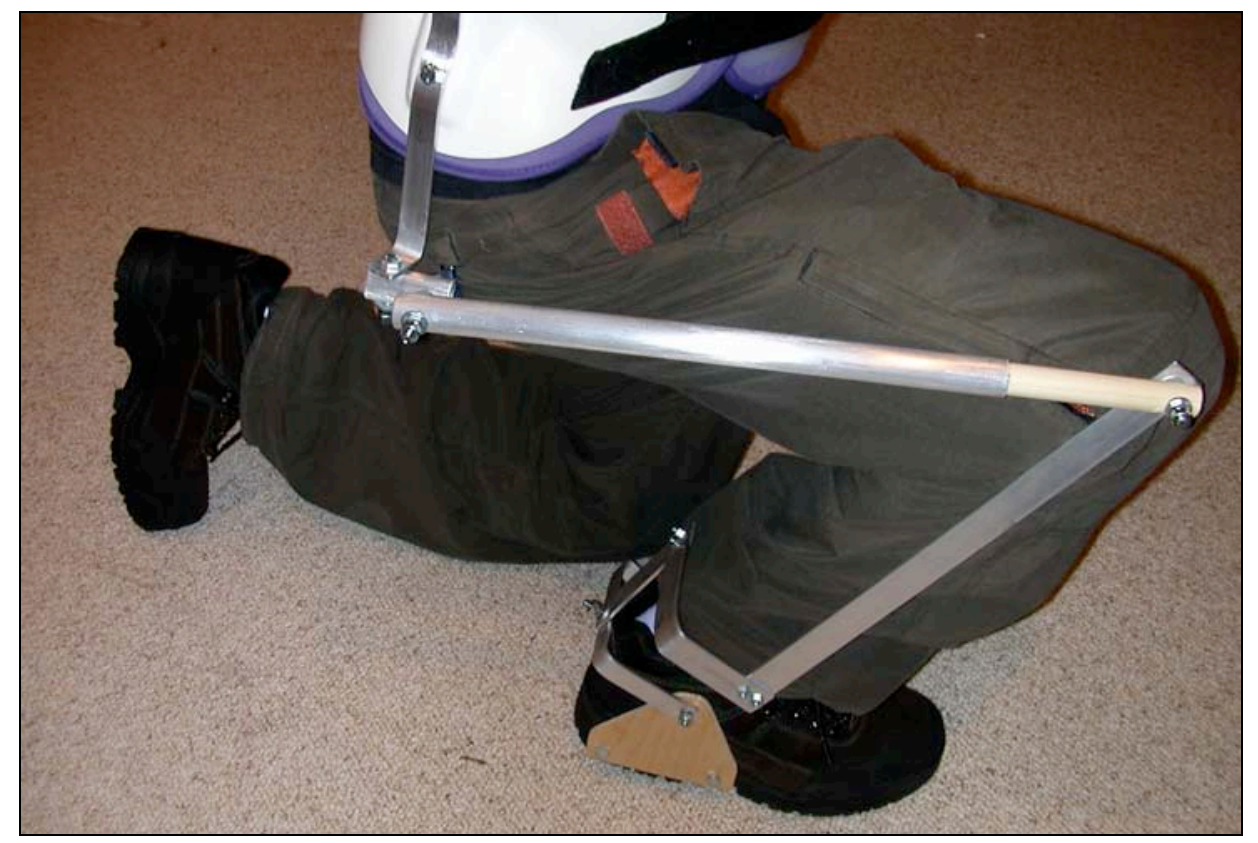

Fig. 38. Deep knee bend.

Figure 39 shows foot inversion while Fig. 40 demonstrates ankle flexion/extension maneuvers. 


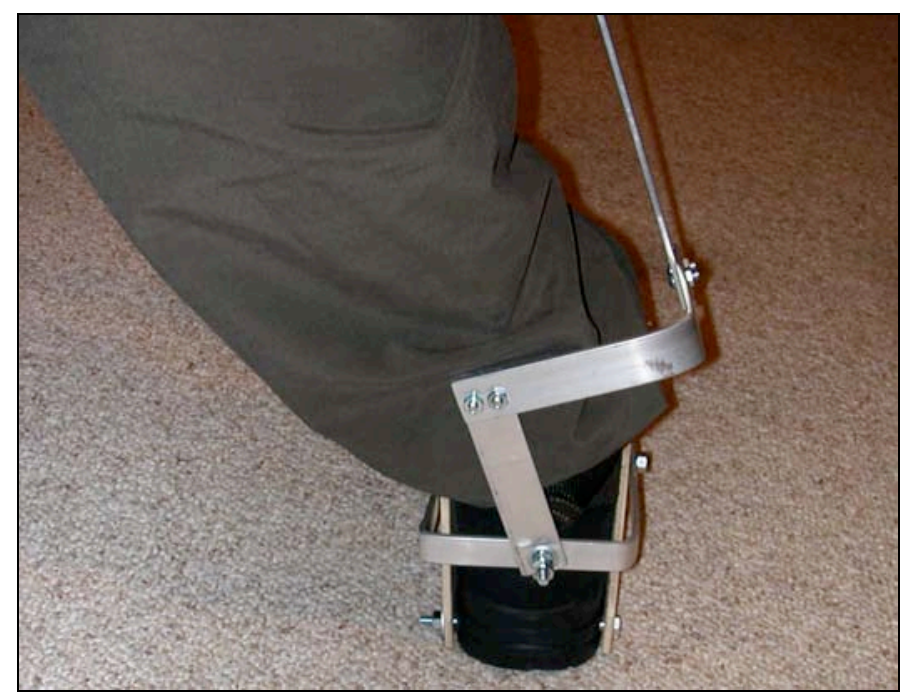

Fig. 39. Foot inversion.
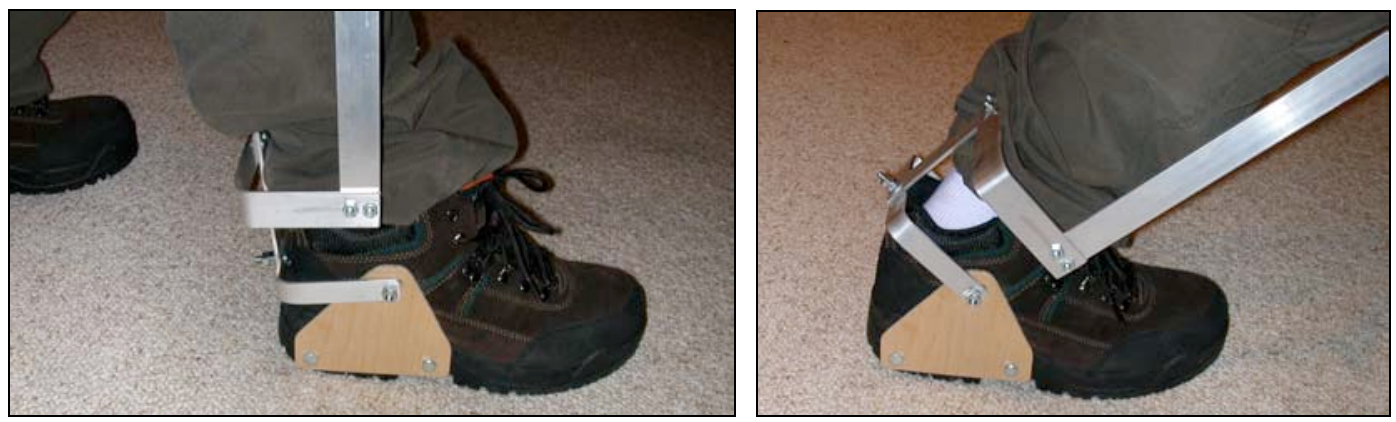

Fig. 40. Ankle flexion/extension motions.

Figure 41 shows hip internal/external rotation and Fig. 42 shows hip flexion/extension motion.
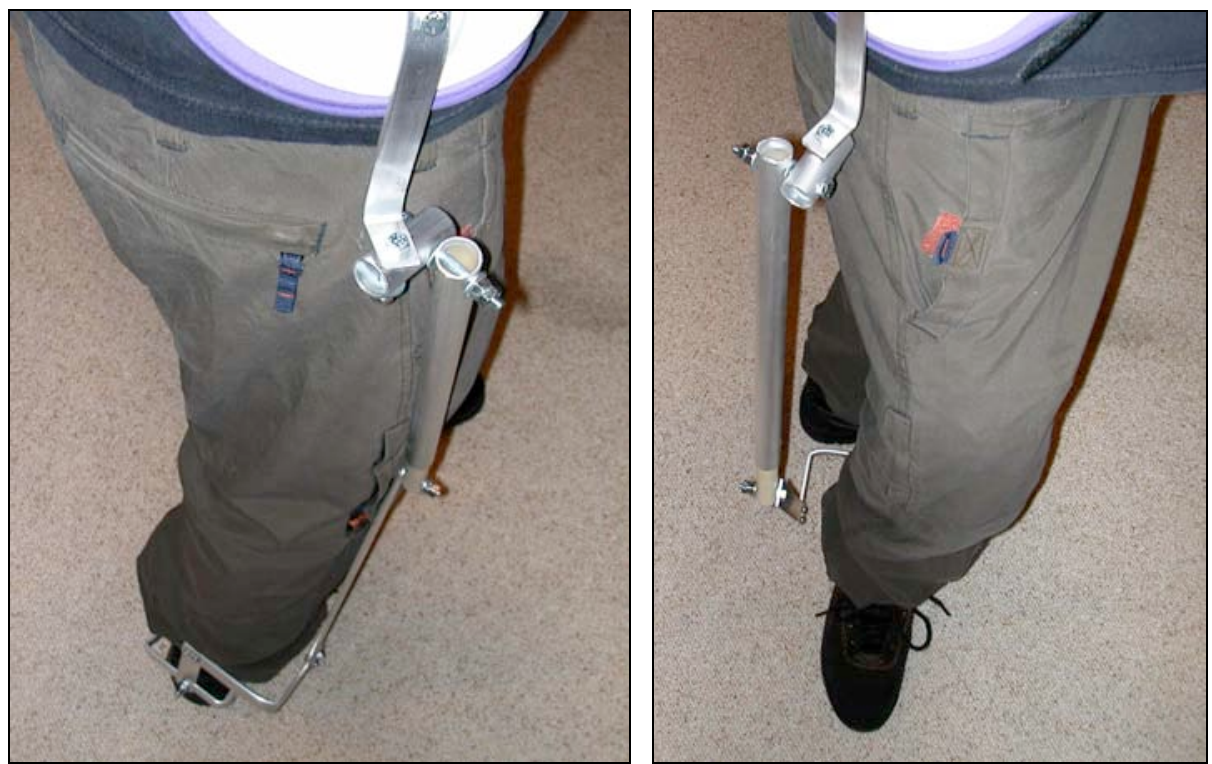

Fig. 41. Hip internal/external rotation. 


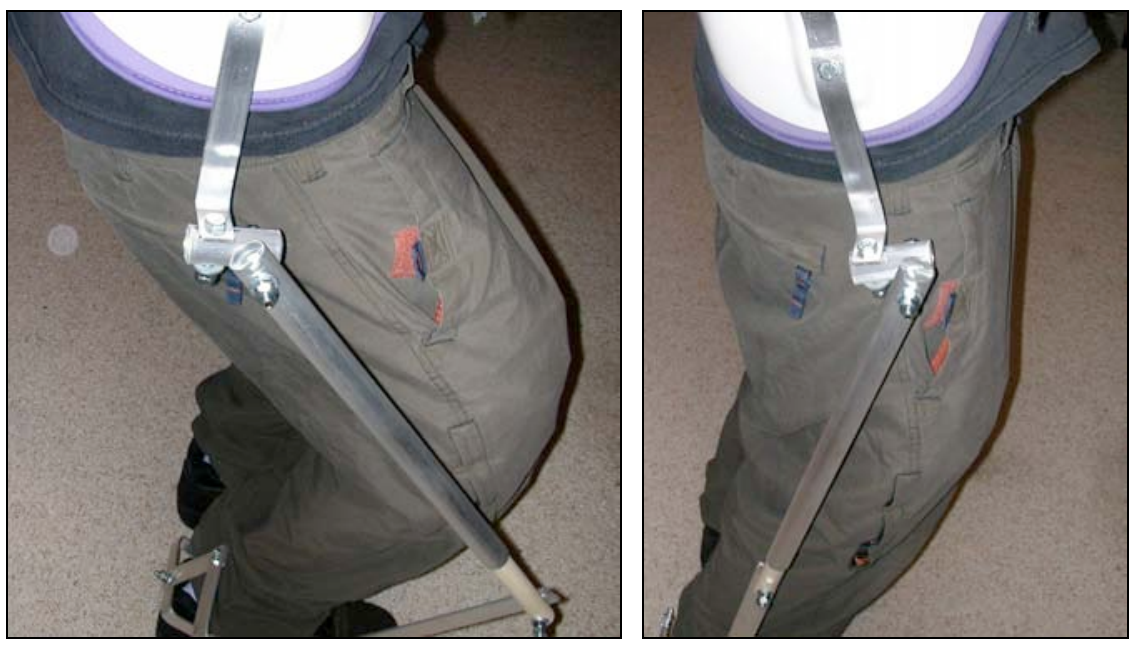

Fig. 42. Hip flexion/extension.

Results from informal wearing of the mockup verified that the kinematic design chosen for the exoskeleton is viable. Comprehensive human factors testing is needed to confirm these results.

\section{FOOT INTERFACE TESTING}

A series of tests have been planned for Phase II, each with another layer of realism added. In the first tests a controlled hydraulic piston will replace the human and will apply accurate force or motion loading profiles to the force sensor. In the second test, a SACH (solid-ankle, cushionedheel) prosthetic foot (Fig. 43) will be driven by the hydraulic piston. The SACH foot, with a compliance and shape that closely match that of a real foot, offers the highest level of realism without using an actual human. The third test will add a human user exercising the system through a wide range of voluntary motions including standing, weight shifting, stepping, and stomping. The motion limits of the exoskeleton will be constrained with limit stops for safety.

The tests are designed to understand controller properties and are not meant to test comfort. An important objective is to determine the range of acceptable compliance for foot padding and ground contact. Real orthotic padding materials will be used in these tests with the goal of determining the types and thickness of padding that are acceptable for stable control. This will establish the constraints for the final boot design.

Force and position data will be collected for all tests. In addition, internal boot loads against the foot would be tested using individual Flexi-force or distributed pressure sensor arrays from Tekscan (Fig. 44).

Future test beds will include a single-leg, multi degree-of-freedom (DOF) system where the leg can move in all directions and where contact stability for all motions of the foot (heel-strike, toeoff, flat foot, inversion/eversion) can be tested. The single-leg, tethered exoskeleton will next be tested during treadmill walking for loaded and unloaded conditions. Testing of a two-leg exoskeleton prototype is further in the future, and will include testing in-place, on a standard treadmill and on the 2-DOF treadmill at ARL. 


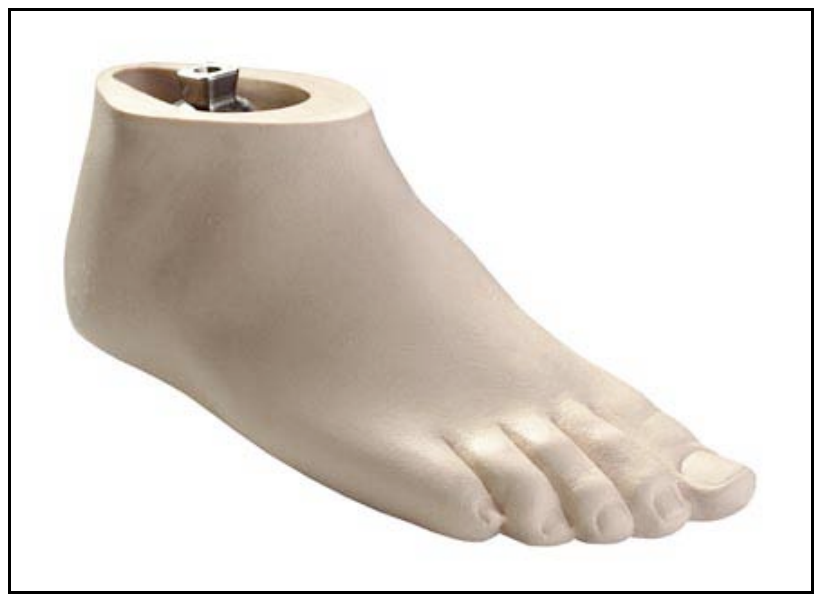

Fig. 43. SACH foot for mannequin testing.

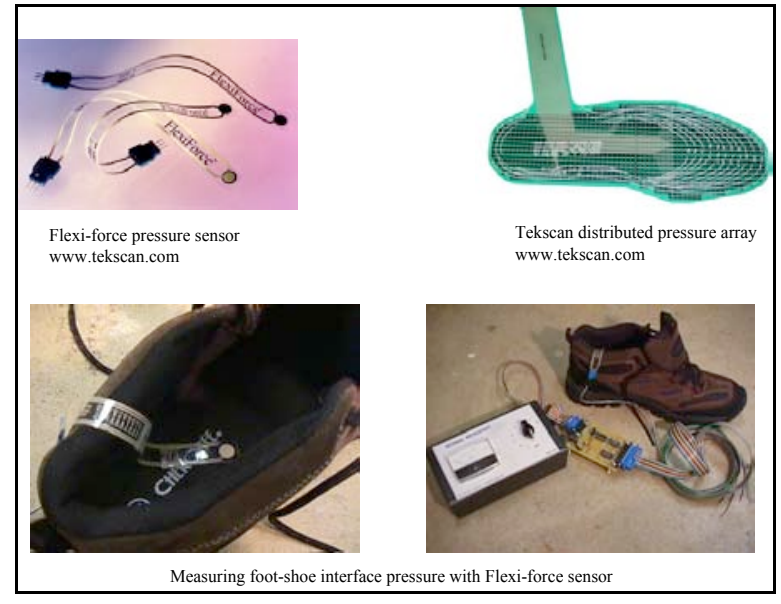

Fig. 44. Testing shoe/foot interface loads. (Testing thin pressure sensors.) 


\section{POWER SYSTEM}

\section{BACKGROUND $\$$}

For an application such as an exoskeleton, there will be significant differences in the steady-state power requirements and the peak power or transient demands. The power source must, therefore, be able to meet both the energy requirements and peak power demands and comparison of potential power sources must be made with respect to meeting both of these requirements. For an exoskeleton type of system, the specific power (power $\div$ weight) and specific energy (energy $\div$ weight) are the parameters that are more useful for comparison of potential power systems. Ragone Diagrams (Fig. 45), which plot specific energy vs specific power, provide a means for making broad, general, comparisons between potential power sources. However, it should be noted that Ragone Diagrams provide information on steady-state specific power and specific energy, but they do not provide any indication as to the response time of a power source and thus, its ability to follow demand.

Figure 45 provides a general comparison of typical fuel cells, batteries, internal combustion engines, and super capacitors. It should be noted that on-going research is continuing to expand the capabilities of these power sources. Although other power sources and storage devices, (e.g., compressed gas, thermoelectric devices, and flywheels) are available, they are not considered practical for a remote mobile device, such as an exoskeleton. From Fig. 45, it can be seen that (1) internal combustion engines have relatively high specific power and specific energy and (2) a hybrid system such as a fuel cell and either batteries or super capacitors can also meet the requirement of high specific power and energy.

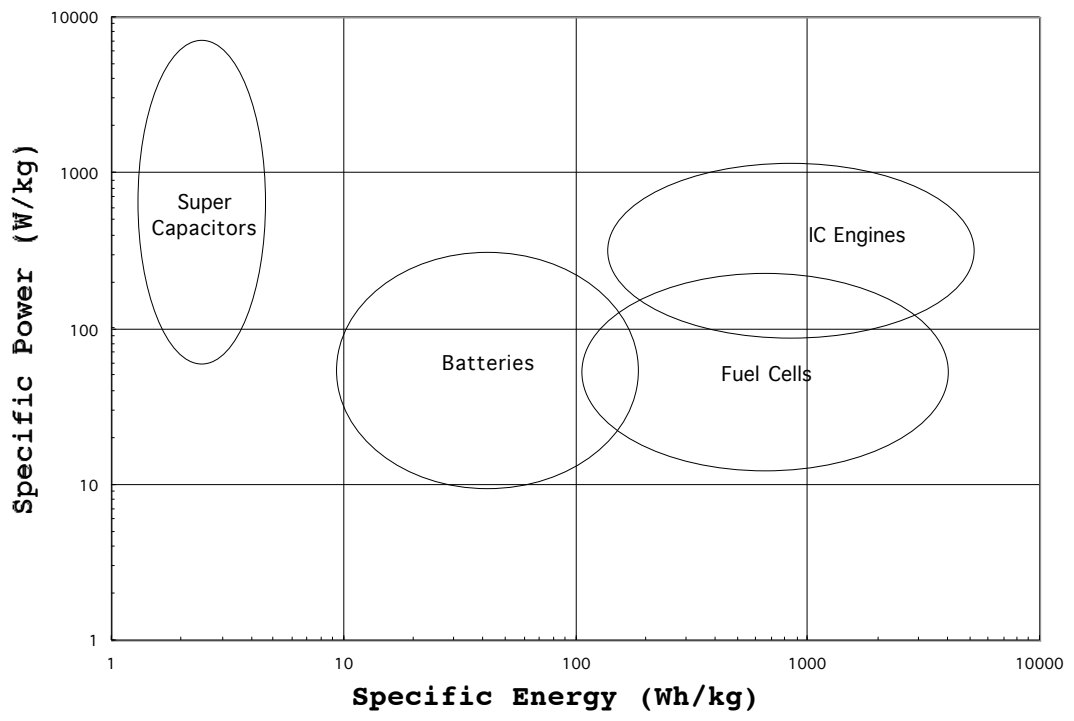

Fig. 45. Comparison of different power sources.

\footnotetext{
${ }^{\S}$ Appendix E provides additional background details regarding the basis of the approach taken (Jansen et al., 2000).
} 
The power source for an exoskeleton must satisfy both power and energy requirements. With the significant variability in anticipated power demands, the ability of the power source to respond to peak loads without impeding performance is essential. Oversizing a single power source to meet both power and energy requirements is not consistent with the need for efficiency required for this application. A hybrid power system, where transient peak power demands are met with one source and mission energy requirements are met with another source, can enhance efficiency at the expense of a more complicated system. Super capacitors and nickel-metal-hydride (NiMH) batteries are both capable of meeting peak transient load requirements, but they are insufficient as an energy source. A fuel cell fed by hydrogen, which is generated by a chemical reaction and an internal combustion engine (ICE) are both capable of meeting system energy requirements.

Table 3 compares the weight for three possible power sources, diesel engine, fuel cell, and batteries, as well as two possible hybrid systems. While the weights for the ICE are lower than those for the fuel cell, it must be noted that the ICE weights do not include provisions for signature (noise, thermal, and emissions) reduction and vibration damping. These can be compensated for, but at the expense of increased complexity and weight. The weight of the fuel cell and the hybrid fuel cell approach are the same. Should a fuel cell itself be capable of responding fast enough to meet peak transient demands, there would be no inherent advantage to the hybrid fuel cell approach over a fuel cell.

Table 3. Power source comparison

\begin{tabular}{|c|c|c|c|c|c|c|}
\hline Power Source Type & $\begin{array}{c}\text { Specific } \\
\text { Power } \\
(\mathrm{kW} / \mathrm{kg}) \\
\end{array}$ & $\begin{array}{c}\text { Specific } \\
\text { Energy } \\
(\mathrm{kWh} / \mathrm{kg}) \\
\end{array}$ & $\begin{array}{c}\text { Energy/ kg } \\
\text { of Fuel } \\
(\mathrm{kWh} / \mathrm{kg}) \\
\end{array}$ & $\begin{array}{c}\text { Weight for } \\
\text { Power } \\
\text { (kg) }\end{array}$ & $\begin{array}{c}\text { Weight for } \\
\text { Energy } 1 \\
(\mathrm{~kg})\end{array}$ & $\begin{array}{c}\text { Total } \\
\text { Weight } \\
(\mathrm{kg}) \\
\end{array}$ \\
\hline Diesel ICE $^{2}$ & $\begin{array}{c}0.4 \\
\end{array}$ & & 3.3 & $\begin{array}{l}5.0 \\
\end{array}$ & 2.4 & 7.7 .4 \\
\hline PEM Fuel Cell ${ }^{2,3}$ & 0.5 & & 1.2 & 4.0 & 6.8 & 10.8 \\
\hline Li Ion Batteries $^{4}$ & 0.5 & 0.25 & & 4.0 & 32.0 & 32.0 \\
\hline $\begin{array}{l}\text { Hybrid } \\
\qquad \begin{array}{l}\text { Fuel Cell } \\
\text { Battery }(\mathrm{NiMH})^{6} \\
\text { Total }\end{array}\end{array}$ & $\begin{array}{l}0.5 \\
1.0\end{array}$ & & 1.2 & $\begin{array}{l}2.0 \\
2.0\end{array}$ & 6.8 & 10.8 \\
\hline $\begin{array}{l}\text { Hybrid } \\
\text { Diesel ICE } \\
\text { Battery (NiMH) } \\
\text { Total }\end{array}$ & $\begin{array}{c}0.4 \\
1.0\end{array}$ & & 3.3 & $\begin{array}{l}2.5 \\
2.0\end{array}$ & 2.4 & 6.9 \\
\hline
\end{tabular}

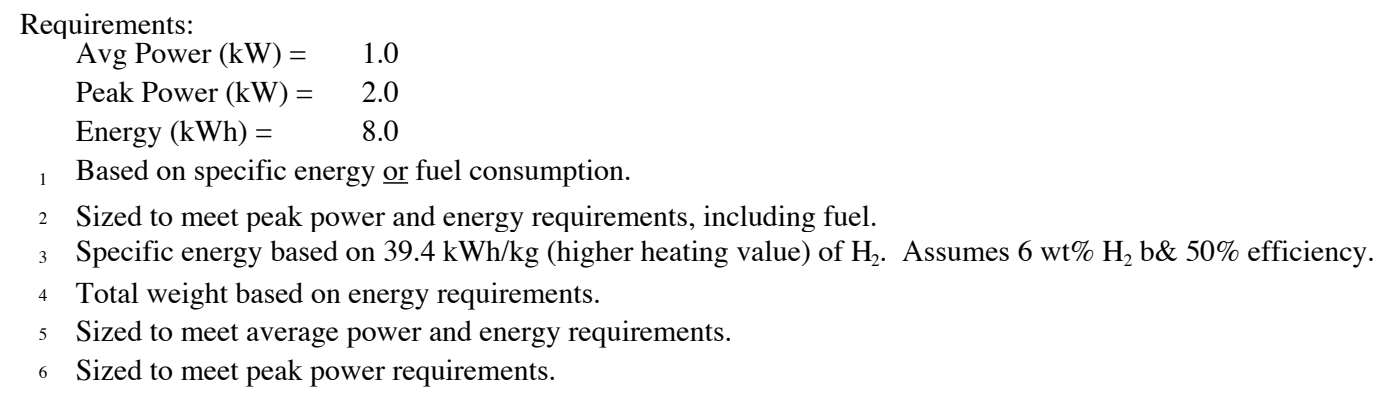

Fuel cells allow for more stealthy operation because of their quiet operation, relatively low temperatures, and benign emissions (water). While there are development issues remaining to be solved for fuel cells, primarily regarding the hydrogen source, there appears to be viable options. 
These are currently being pursued by commercial suppliers and are already being introduced into the market for niche applications. It should be noted that there have been significant increases in fuel cell power density, with more increases anticipated. Integrating a super capacitor or NiMH batteries for transient response with a fuel cell fed by chemically generated hydrogen offers the potential for best meeting the combination of power, energy, and signature objectives and safely function in close proximity to a human operator. This is the approach (Fig. 46) that has been taken in Phase I and will be discussed in greater detail.

The focus of the Phase I power supply effort was on demonstrating a bench top integrated, hybrid power source that would meet both energy and power needs (including peak transient loads) for an exoskeleton. The system was successfully designed, fabricated, and tested. In addition, the feasibility of water recycling as a means of increasing the energy density was also investigated. Both of these efforts are described in detail below.

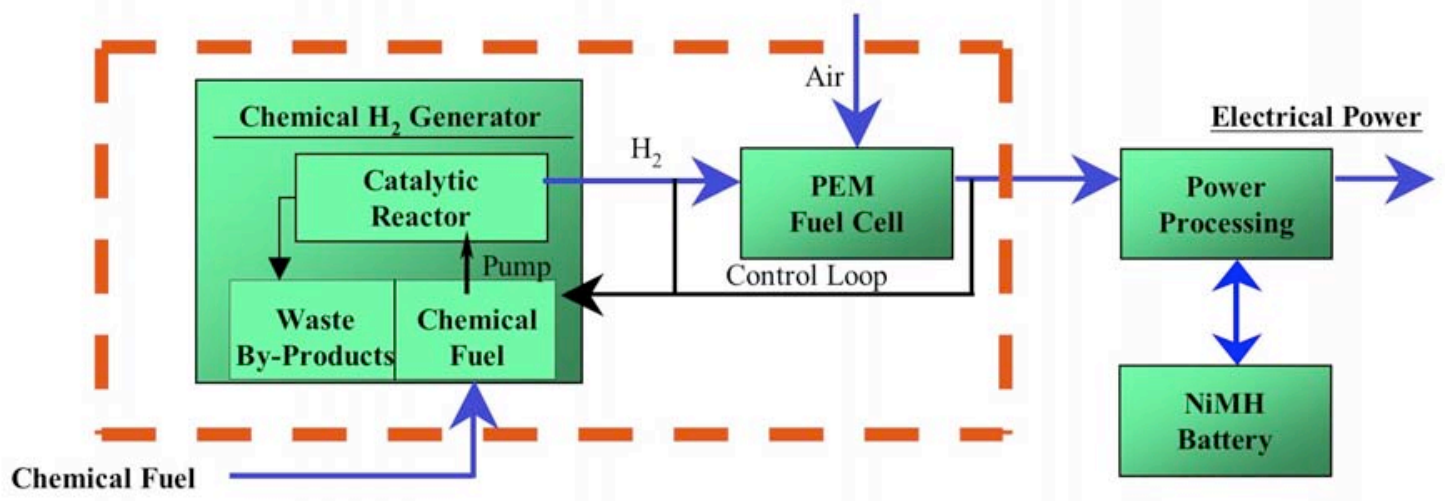

Fig. 46. Power system block diagram.

\section{INTEGRATED POWER SYSTEM ${ }^{\text {*** }}$}

The power source tested was comprised of three basic parts: (1) a proton exchange membrane, hydrogen-air fuel cell as the base power requirement; (2) a battery transient response module to address situations when the power requirements exceed the power deliverable by the fuel cell or in cases where the fuel cell response lags the load, and; (3) an easy method to generate hydrogen, control its flow to the fuel cell, and recycle its by-product.

A five-step plan was employed to allow rapid construction of a demonstration power supply for our chosen hybrid architecture. The first step was to select off-the-shelf components. The second was to design and build the power supply. Third, the power supply was tested, debugged, and integrated into the system. The fourth step was to develop the system controller and the additional hardware to measure and control all system parameters. In the fifth step, the ORNL chemical hydrogen generation subsystem was integrated and the sodium borohydride solutions prepared. The integrated system was fabricated and tested under several upper- and lower-bound power profiles.

\footnotetext{
*** This section summarizes the report documented in AeroVironment, 2003, and found in Appendix F.
} 
The result of this approach was a prototype system ready to operate from fuel to electricity and demonstrating hybrid system capabilities for the soldier's energy and power requirements. An operating voltage of 48VDC was selected as best for control of the hybrid system. From this voltage, a system control concept was developed and a control circuit was designed. NiMH batteries were selected for the transient handler system. Demonstrations used hardware to analyze and understand the nuances of the chosen hybrid power architecture-placing paramount emphasis on robustness, simplicity, safety, and system sizing flexibility-related issues.

A hybrid system-sizing map (the parameter space) showed optimized system configuration for soldier energy demand average, peaks, and down time (available recharge time). No single power profile could determine base system sizing criteria because a soldier's activity is varied. With the help of ORNL, models were developed for a variety of scenarios and analyzed from a system sizing perspective. Most significantly, a demonstration (portable) system operating under automatic control, from fuel to electricity, was built by adding a hydrogen storage and handling system from ORNL. The system demonstrated the viability of the control concept through both steady state and transient operating parameters defined in the modeling task. It also tested the performance and the transient handling capability of the ORNL hydrogen generator as a fuel source to the baseline hybrid fuel cell system.

The final product was a self-controlled, self-contained system that starts with a push of a button. Figure 47 illustrates best the overall concept. The PC is used for data display and recording only. The electric load is on the bottom shelf and is used for system testing. The fuel cell stack is an off-the-shelf system and no effort has been expended in reducing the size of the stack or the balance of plant components. The whole system is spread out and is not optimized for weight or size. As it is, it can easily be scaled depending upon power needs and energy storage requirements.

The data in Fig. 48 illustrate how the system can deliver peak power to the electric load as needed. When the transient handler state-of-charge dips below $20 \%$ the fuel cell starts charging it, whenever there is excess power available.

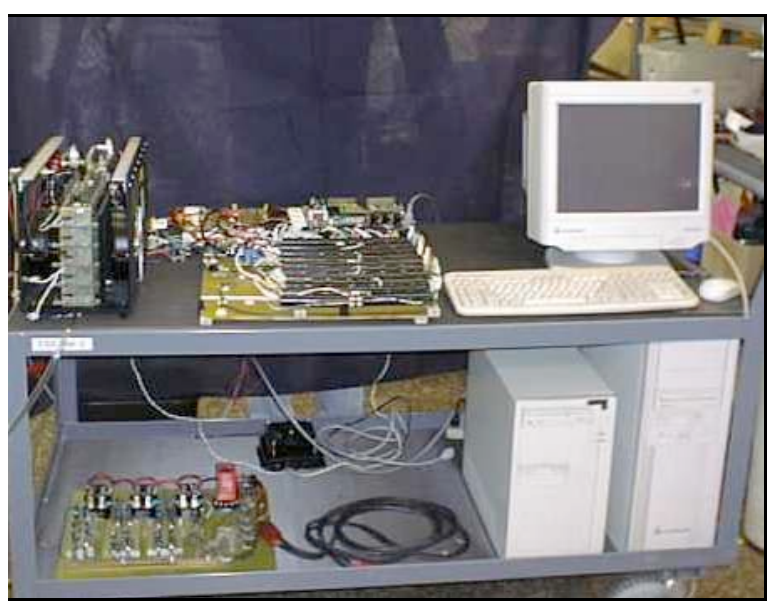

Fig. 47. Integrated power test bed.

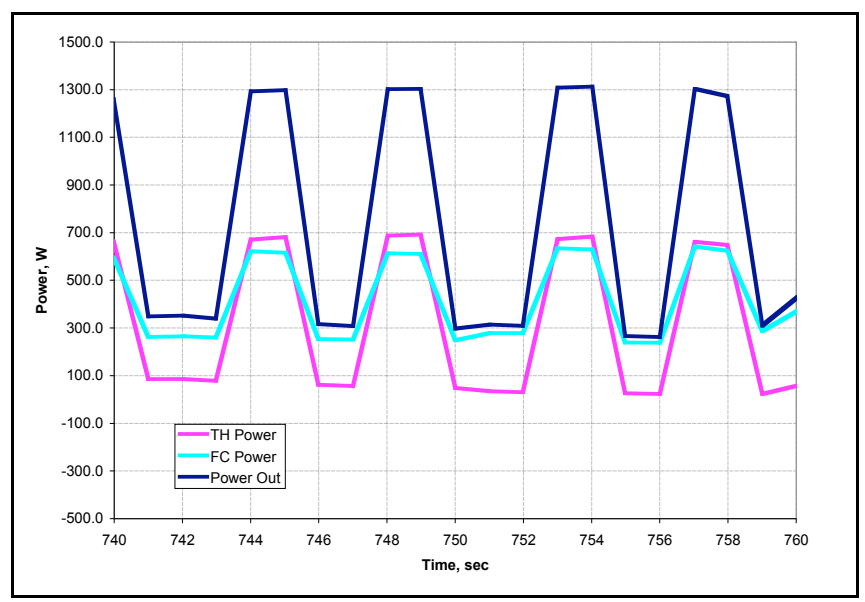

Fig. 48. Power testbed experimental data. (Fuel cell and transient handler delivering $1.3 \mathrm{~kW}$ pulsed power.) 
Figure 49 shows the power electronics module that is responsible for regulating the output of the energy storage system and controlling the state of charge for the transient handler. Figure 50 is a picture of the system controller. Figure 51 shows the hydrogen generation module, which consists of a catalytic reactor, developed at ORNL, a spent solution chamber and a fresh solution chamber. A small pump pushes fresh $\mathrm{NaBH}_{4}$ solution ( $<17 \% \mathrm{NaBH}_{4}$ in water) through the catalytic reactor. Hydrogen produced is cooled off to stack temperature and fed to the fuel cell. Hydrogen pressure drops prompt new solution to be pumped into the catalytic reactor. The spent solution accumulates in a reservoir, also used as a phase separator, and prevents liquid from entering the fuel cell stack. A fine mesh filter is also used to make sure that the produced hydrogen does not carry any small liquid particles into the fuel cell stack. An off-the-shelf compressor provides air needed for fuel cell operation. The Battery System Controller (BSC) controls its speed, proportional to the fuel cell stack current, and fuel cell stack temperature. Three fan speeds are used, to conserve energy.

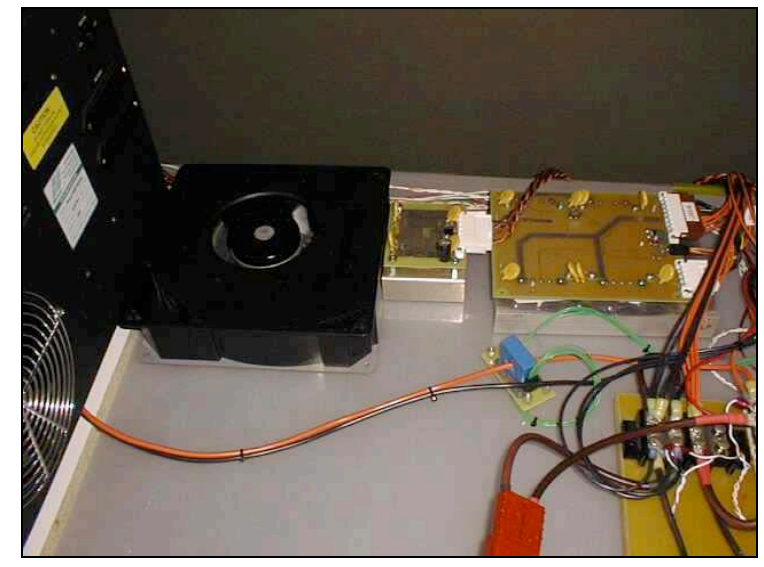

Fig. 49. Power electronics module.

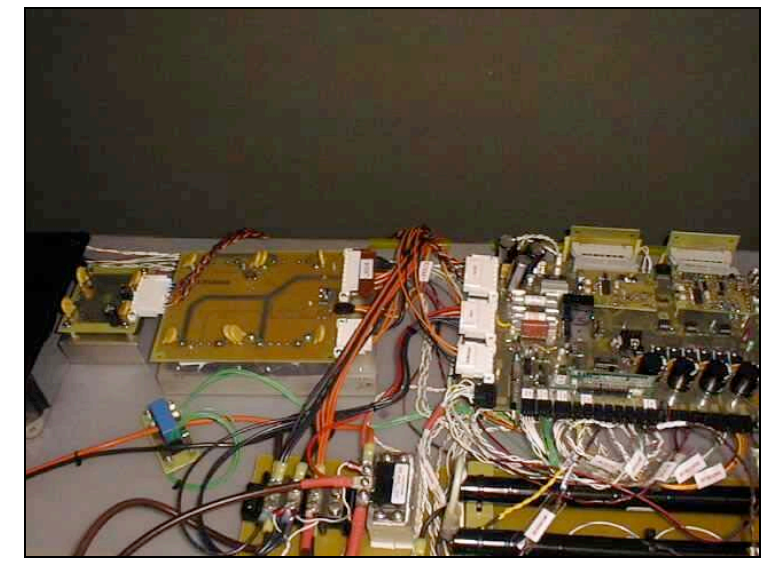

Fig. 50. Power system controller module.

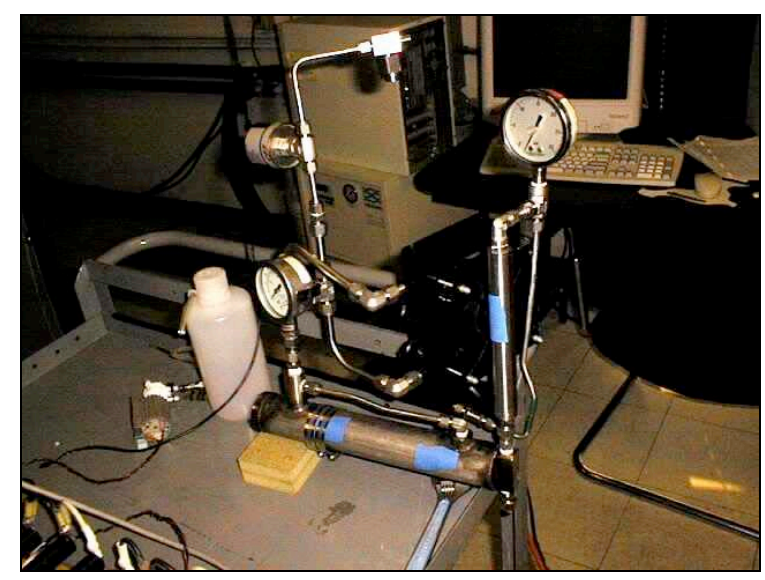

Fig. 51. Hydrogen generation module.

Figure 52 shows the fuel cell stack module with a data acquisition system that measures cell voltage in the stack. The system consists of five FC-SG modules, of 12 channels each. The modules are connected to the system controller via a fast serial bus. The data acquisition system was useful during testing but may not be needed when it is fully developed. Figure 53 shows the 
transient handler module, instrumented with voltage and temperature measurement. It is monitored and controlled by the BSC with an elaborate algorithm that inputs temperature, Ah depletion/addition, and average cell voltage.
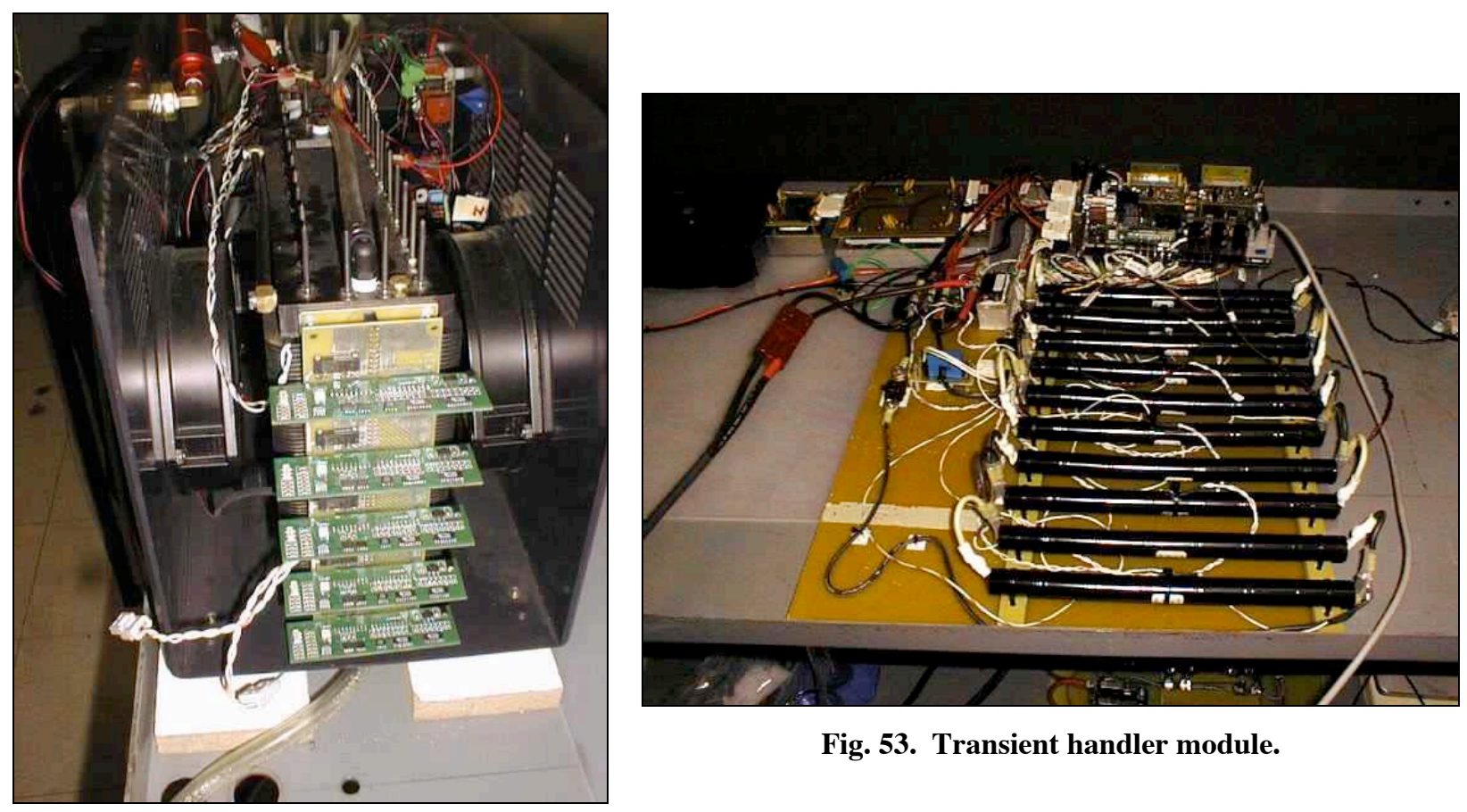

Fig. 53. Transient handler module.

Fig. 52. Fuel cell module.

\section{HYDROGEN SOURCE}

Mobile and portable power systems, such as required for this application, require high-density energy sources to operate efficiently. One option for achieving this efficiency is to store hydrogen in a hydrolysable compound, couple the hydrolysis reaction with a fuel cell, and recycle excess hydrolysis water and fuel cell product water to the hydrolysis reaction. To maximize net energy production, it is necessary for the reaction to occur exothermically. Selection of hydrides with low molecular-weight, high valence cations are essential to maximize the weight and mole fractions of hydrogen in the feed to the hydrolysis reactor. Reaction systems with fast kinetics are preferable since they facilitate hydrogen generation that is responsive to changes in power demand. Finally, use of a stable hydride that undergoes hydrolysis only in the presence of a catalyst is beneficial from safety and controllability standpoints.

Sodium borohydride was discovered in the 1940s and was first investigated as a portable source of hydrogen for balloons by the U.S. Army Signal Corps (Beatty, 1944; Schlesinger, 1944). The compound has also been investigated as a propellant for rocket engines by various researchers (Beatty, 1944). At present, $\mathrm{NaBH}_{4}$ is used primarily as a reductant in the synthesis of organic chemical compounds and as a bleaching agent in the manufacture of paper. 
Sodium borohydride reacts spontaneously with water to generate hydrogen according to the reaction

$$
\mathrm{H}_{2} \text { Generation: } \quad \mathrm{NaBH}_{4}+2 \mathrm{H}_{2} \mathrm{O}+\mathrm{xH}_{2} \mathrm{O} \rightarrow 4 \mathrm{H}_{2}+\mathrm{NaBO}_{2}+\mathrm{xH}_{2} \mathrm{O}
$$

The overall reaction occurs at a moderate rate initially, quickly slows, and then ceases as the concentration of the basic borate ion in the aqueous product solution increases. Researchers have determined that the duration of the reaction can be increased by the addition of acid to the system to counter the base effect of the borate ion (Schlesinger, 1953). The rate of the reaction and the extent to which the reactants are converted to products can be increased significantly in the presence of catalysts. Specific catalysts that have been investigated include various metal chlorides, noble metals, and precipitates that are formed when metal chlorides are reacted with sodium borohydride solutions (Levy et al., 1960; Kaufman and Sen, 1985; Brown and Brown, 1962). Ruthenium has been found to be the most effective catalyst for promoting hydrogen generation (Brown and Brown, 1962). In our previous work, we confirmed that the hydrolysis of $\mathrm{NaBH}_{4}$ in aqueous solution is initiated quickly under ambient conditions and proceeds to $100 \%$ conversion when the reaction is catalyzed by activated ruthenium. We also determined that the reaction ceases quickly when reactants and catalyst are separated, or when reactant flow through a packed catalyst chamber is terminated, which is extremely important for controllability.

At present, all proposed applications of $\mathrm{NaBH}_{4}$-based hydrogen generation have been based on reactions using aqueous feed solutions. Because of the limited solubility of the reaction product salt, sodium metaborate, the concentration of $\mathrm{NaBH}_{4}$ in the aqueous solution is limited to approximately $30 \mathrm{wt} \%$. As indicated by the stoichiometry in Eq. (1), the hydrolysis reaction derives half of the product hydrogen from solution water, effectively doubling the storage density of the hydride. Consequently, the effective weight percentage of hydrogen in the feed solution is limited to $6.4 \mathrm{wt} \%$. In addition, a basic compound (e.g., sodium hydroxide) must be added to the reagent solution to suppress uncatalyzed hydrolysis in the stored reactor feed to prevent loss of hydrogen. The suppressant fraction added to the feed solution replaces part of the water fraction, thereby impacting the solubility of the reaction product and forcing a reduction in the concentration of $\mathrm{NaBH}_{4}$ solution in the feed. As a result, the practical hydrogen concentration in the borohydride solution is limited to about $4 \mathrm{wt} \%$.

The catalyzed hydrolysis of $\mathrm{NaBH}_{4}$ is sufficiently exothermic to vaporize most of the excess water in the feed solution. Vaporization of excess water facilitates its separation from the $\mathrm{NaBO}_{2}$ waste exiting the reaction chamber. Coupled with the fact that a fuel cell produces water as a by-product, Eq. (2), it is theoretically possible to continuously segregate and recycle the water in a $\mathrm{NaBH}_{4} /$ fuel cell system. In fact, since for every mole of water consumed in the hydrolysis reaction, the reaction at the fuel cell produces 2 moles of water, the system is a potential net generator of water.

$$
\begin{aligned}
& \text { Fuel Cell: } \quad 4 \mathrm{H}_{2}+2 \mathrm{O}_{2} \quad \rightarrow \quad 4 \mathrm{H}_{2} \mathrm{O}
\end{aligned}
$$

Water recycling eliminates the need for on-board storage of any significant volume of water, since solid $\mathrm{NaBH}_{4}$ reactant can be dissolved on demand into a small volume of water that is continuously replenished during system operation. Consequently, the effective hydrogen storage 
density of the fuel is $21 \mathrm{wt} \%$ : one half of which comes from $\mathrm{NaBH}_{4}$ and half from the recycled water. With water recycling and the resulting elimination of significant aqueous $\mathrm{NaBH}_{4}$ solution storage, the addition of a suppressant to prevent uncatalyzed hydrolysis is minimized or eliminated.

As discussed previously, there are two potential sources of water, the fuel cell and the hydrogen generation process itself (Eqs. (1) and (2)). The percentage of the available water that must be recovered for full water recycling (i.e., no external water added, dry $\mathrm{NaBH}_{4}$ used) is shown in Fig. 54. The minimum line represents the best-case scenario, which will be discussed in greater detail in the next section. It can be seen that based on the minimum line, and with a $25 \% \mathrm{NaBH}_{4}$ solution, $\sim 80 \%$ of the available water must be recovered for full water recycling. A series of experiments were set up and conducted for the purpose of evaluating and demonstrating the feasibility of water recycling. Both the recovery of water from the fuel cell and the hydrogen generation were addressed in these experiments.

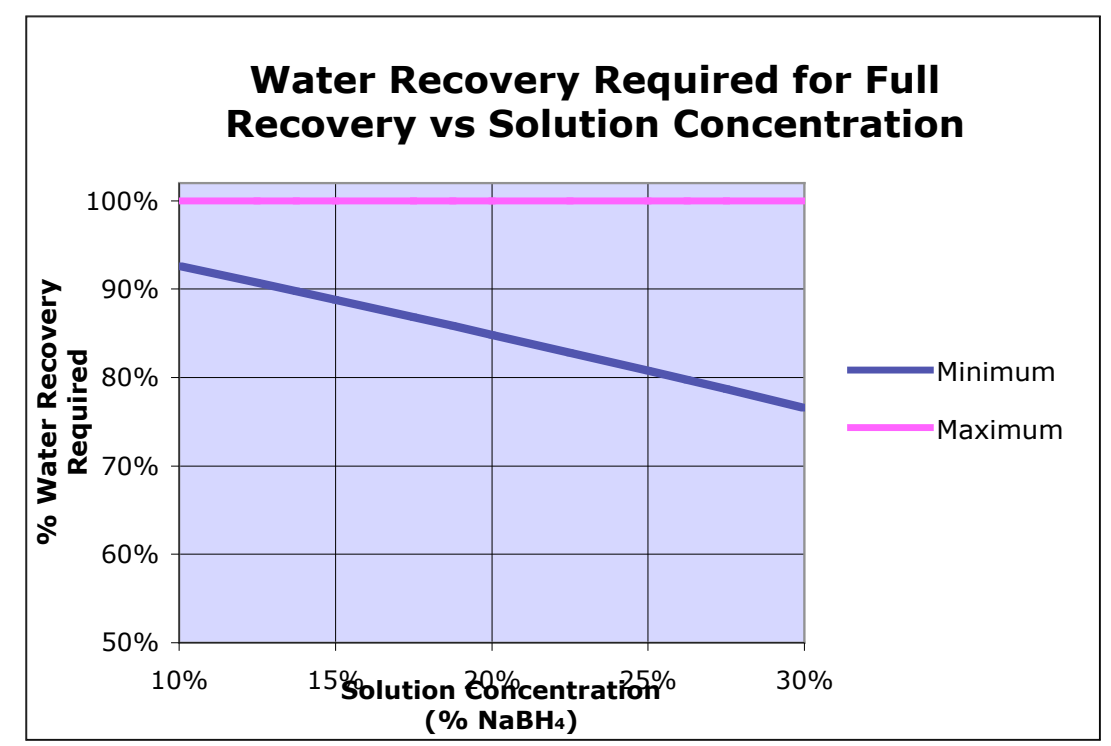

Fig. 54. Water recovery versus concentration.

\section{Fuel Cell Water Recovery}

As was seen in Eq. (2), for every molecule of $\mathrm{H}_{2}$ used by the fuel cell, one molecule of $\mathrm{H}_{2} \mathrm{O}$ is generated. Capturing this water provides the opportunity to replace the water consumed in the hydrogen generation reaction (Eq. (1) and potentially to make the system a net water generator. To test how much water could be recovered from the fuel cell an experiment was set up according to Fig. 55. Bottled hydrogen was fed to a commercial fuel cell rated at $500 \mathrm{~W}$ that was connected to a simulated load. The output from the fuel cell drain was collected and measured. 
Based on these measurements, $>80 \%$ of the available water was collected. As discussed above, the quantity of water needed for full water recycling with a $25 \mathrm{wt} \%$ feed solution is $80 \%$. Based on this simple lab bench test, the recovery of water from the fuel cell is consistent with this target.
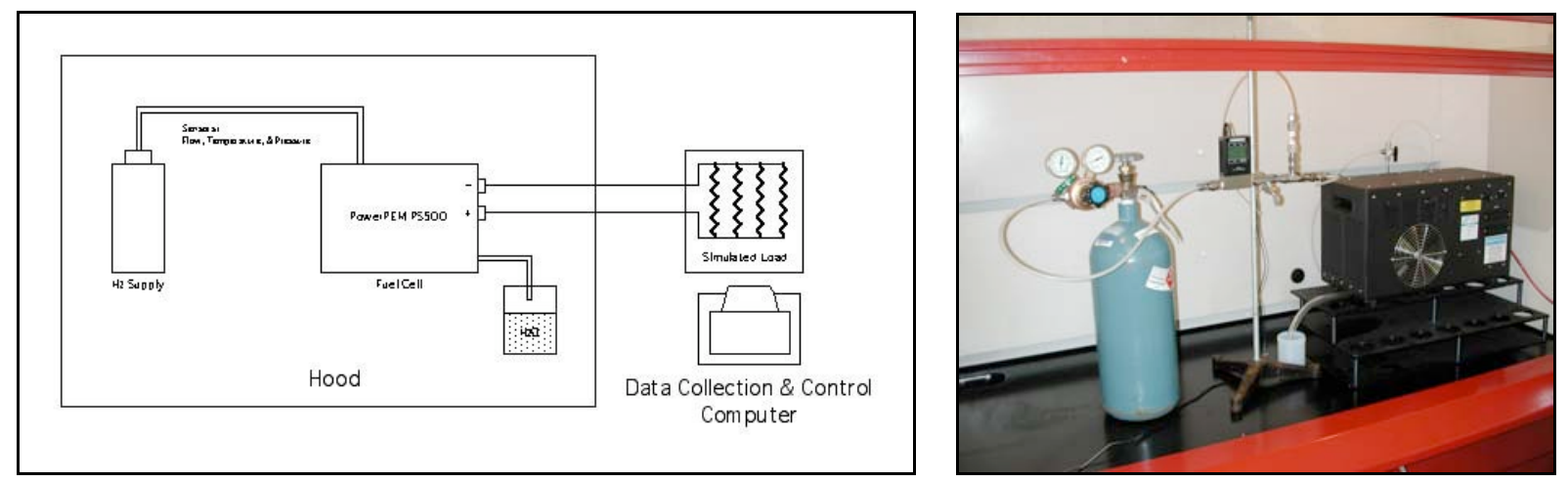

Fig. 55. Fuel cell water recovery test setup.

\section{$\mathrm{H}_{2}$ Generation Water Recovery}

For a $25 \mathrm{wt} \% \mathrm{NaBH}_{4}$ solution, there is a total of 6.3 molecules of water per molecule $\mathrm{NaBH}_{4}$. Two of those molecules are consumed in the hydrolysis reaction (Eq. 2), leaving a maximum of 4.3 available for recovery. If all the water was vaporized in the reaction leaving the sodium borate by-product as a solid, plugging of the reaction chamber would obviously occur. Therefore, while it is desirable to recover as much water as possible, it is not possible to recover all of the available water without plugging the reactor. Preliminary testing led to focusing on the form of the sodium borate by-product in order to strike an appropriate balance between recovering as much water as possible and maintaining flow through the reactor.

The form of the by-product that is produced is one of the key issues in obtaining full water recycling. As can be seen from Fig. 56, sodium borate is more hygroscopic at lower temperatures. Operating above $103^{\circ} \mathrm{C}$ provides the opportunity to generate sodium meta-borate hemi-hydrate $\left(\mathrm{NaBO}_{2} \bullet 0.5 \mathrm{H}_{2} \mathrm{O}\right)$ with only one half molecule of water per molecule of sodium borate as opposed to two or four molecules at lower temperatures. This enhances the ability to recover the higher percentage of water needed for full water recycling. The boiling temperature of the sodium meta-borate hemi-hydrate is $120.2^{\circ} \mathrm{C}$. Operating above this temperature while releasing more water vapor will increase the likelihood of plugging. 


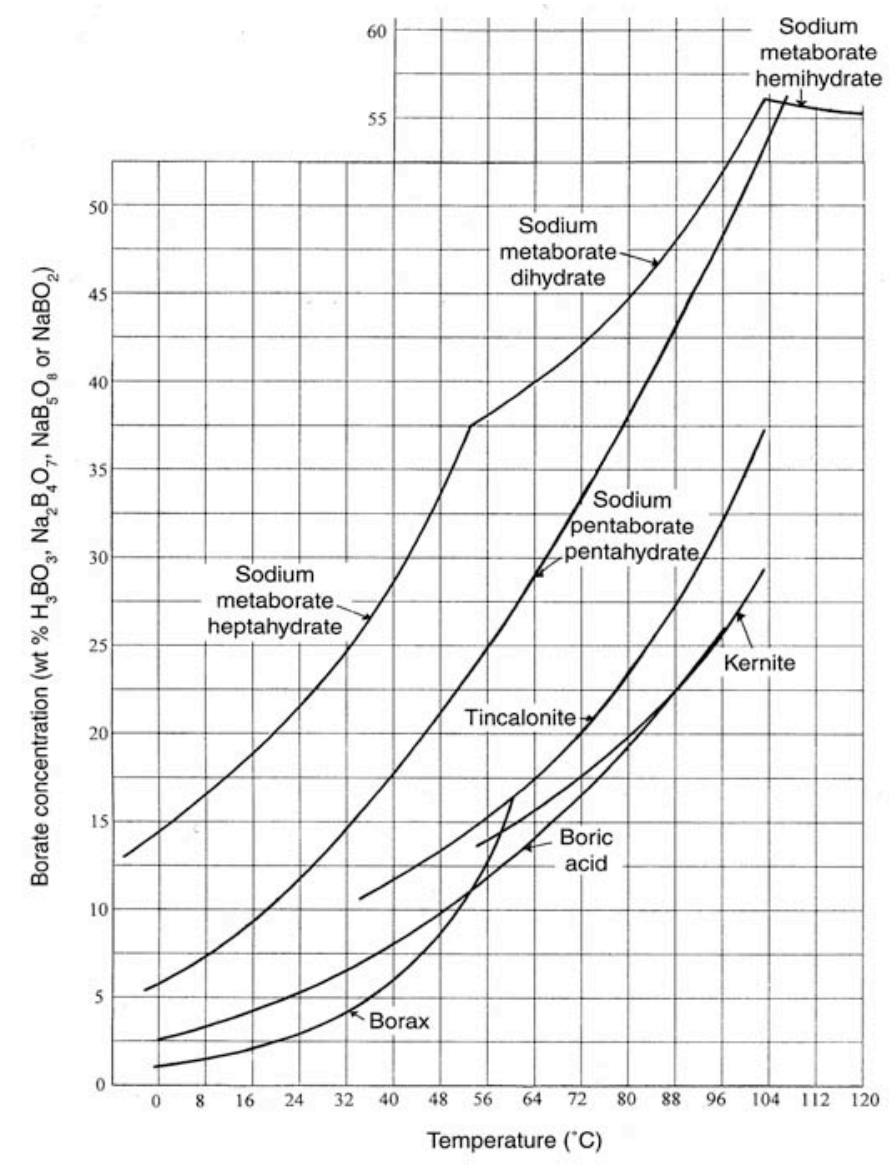

Fig. 56. Solubility of boric acid and several sodium borates. ${ }^{15}$

One of the testing setups for measuring the amount of water that could be recovered from the hydrogen generation reaction is shown in Fig. 57. (Later testing used different vessels that were smaller and much better with respect to leaks, as well as a different reactor design.) The test setup consisted of a hydrogen generator, feed pump, vessels to catch the reaction by-product, a heat exchanger to condense the water vapor, and a vessel to catch the condensate. The generated $\mathrm{H}_{2}$ was vented into the hood.
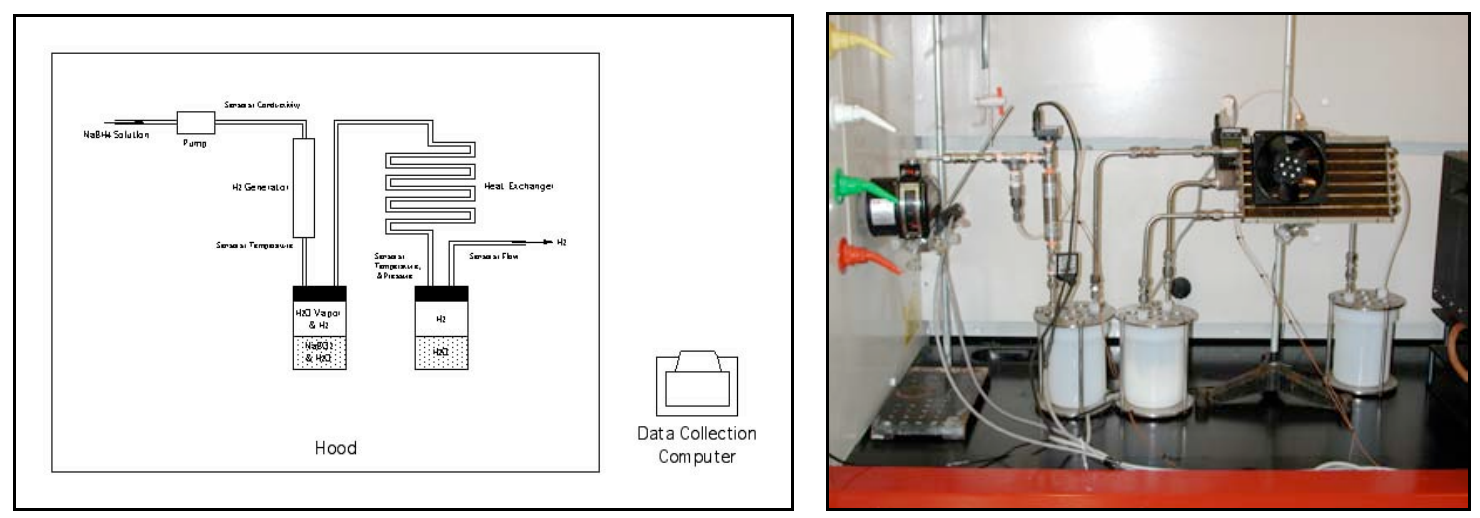

Fig. 57. $\mathrm{H}_{2}$ generation water recovery test setup. 
Water recovery test results are listed in Table 4. Two different generator configurations (Fig. 58) were tested along with a glass generator (Fig. 59) for visualization purposes. In addition, tests were conducted with and without preheating. As the tests were of relatively short duration (on the order of 20 minutes), a relatively large percentage of the total test time $(\sim 25 \%)$ was spent reaching thermal equilibrium. During this transition, the temperature was insufficient to vaporize the excess water, thus limiting the amount of water available for recovery. Tests were also conducted where the system was preheated to the equilibrium temperature to simulate longer duration steady state operation. The results with no pre-heat, where the time spent reaching equilibrium was a high percentage of the overall test time provides more of a worstcase scenario. The results of these tests are encouraging and indicate that full water recycling (i.e., addition of no external water) is feasible. Figure 60 shows the results plotted with the theoretical amount of water recovery needed versus solution concentration.

Table 4. Hydrogen generation water recovery data

\begin{tabular}{|c||c|c|c|c|}
\hline \multicolumn{1}{|c|}{ Test } & \multicolumn{1}{c}{ Generator } & Preheat & Concentration & $\mathrm{H}_{2} \mathrm{O}$ Recovered \\
\hline \hline \multirow{1}{*}{$\mathbf{1}$} & Can & No & $23.1 \%$ & $76.5 \%$ \\
\cline { 2 - 5 } & Cylinder & No & $22.9 \%$ & $65.8 \%$ \\
\cline { 2 - 5 } $\mathbf{3}$ & Cylinder & No & $22.9 \%$ & $70.2 \%$ \\
\cline { 2 - 5 } $\mathbf{4}$ & Cylinder & $230^{\circ} \mathrm{F}$ & $20.0 \%$ & $86.8 \%$ \\
\cline { 2 - 5 } $\mathbf{5}$ & Cylinder & $230^{\circ} \mathrm{F}$ & $20.0 \%$ & $96.1 \%$ \\
\hline
\end{tabular}
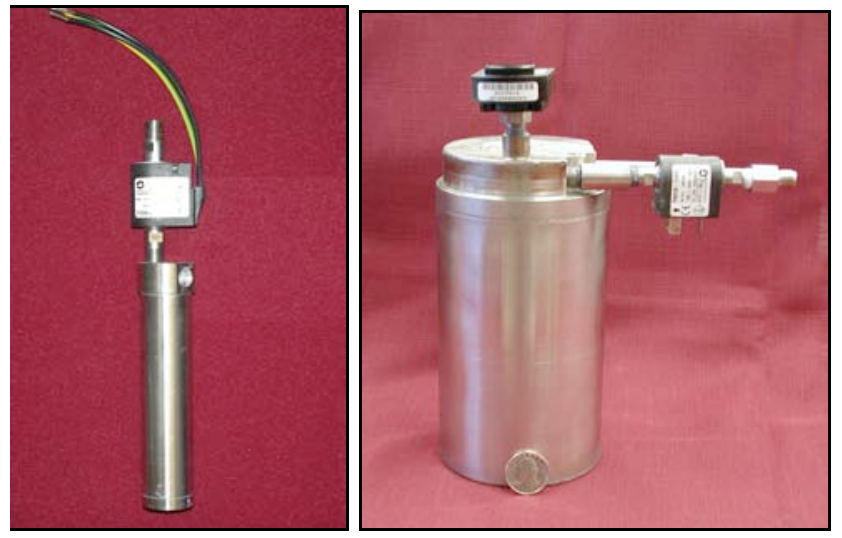

Fig. 58. ORNL $\mathrm{H}_{2}$ generators.

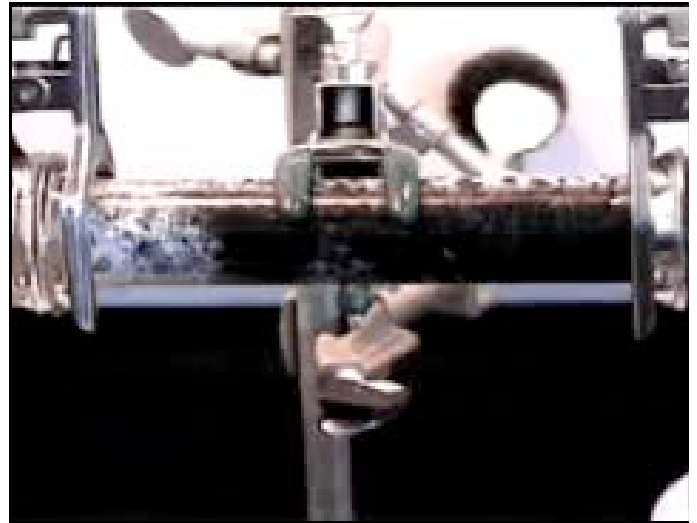

Fig. 59. $H_{2}$ generation in glass generator. 


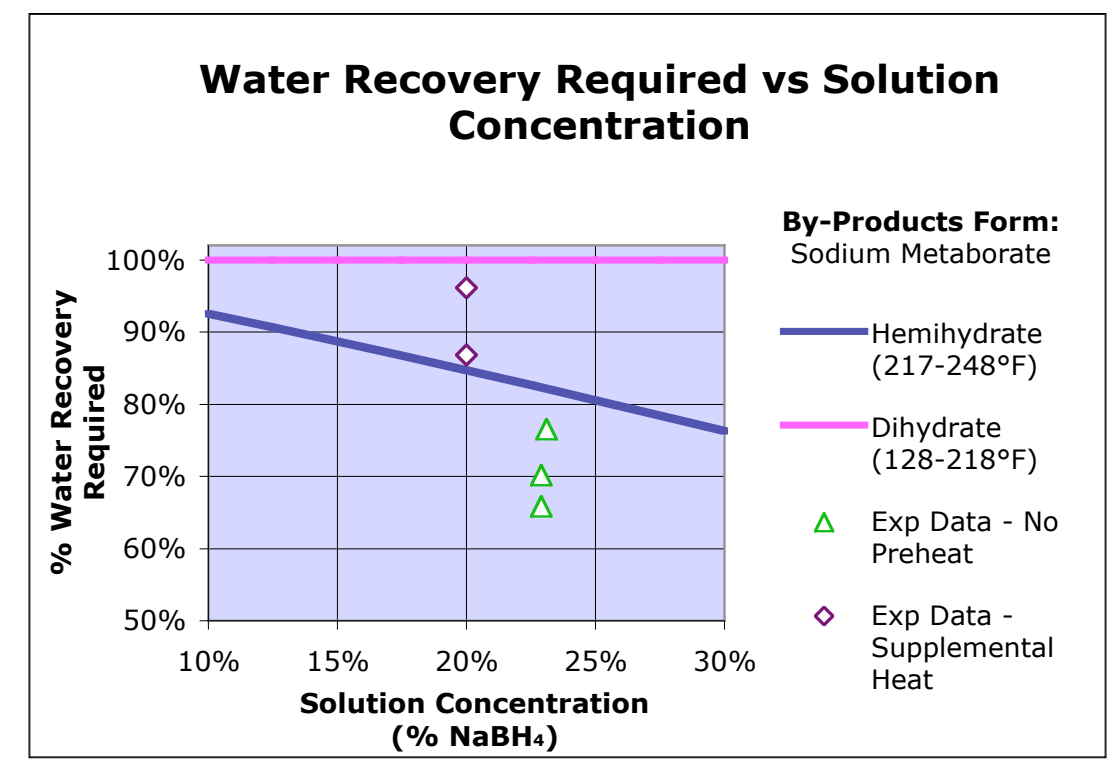

Fig. 60. Test data and water recovery versus concentration.

\section{Other $\mathrm{H}_{2}$ System Testing}

In addition to the water recycle testing, testing was conducted to develop understanding on a few key areas relative to implementing a full system. These tests focused on determining how fast sodium borohydride could be expected to dissolve, how much hydrogen would be lost through off-gassing, and how fast the hydrolysis reaction takes place.

\section{Dissolution Rates}

There are several parameters that affect the dissolution rates, including the concentration of sodium borohydride, the concentration of sodium hydroxide, and the temperature. The solubility of $\mathrm{NaBH} 4$ is shown in Fig. 61 . Below $36.4^{\circ} \mathrm{C}$ represents the solubility of the dihydrate form and above $36.4^{\circ} \mathrm{C}$ the anhydrous form.

A series of measurements were taken to study parameters affecting the rate at which sodium borohydride can be put into solution and are shown in Fig 62. For the solutions containing sodium hydroxide, the sodium hydroxide was dissolved prior to the addition of the sodium borohydride. As anticipated, higher concentrations of sodium borohydride and the presence of sodium hydroxide both slowed the dissolution. It is also evident that as the temperature approaches $150^{\circ} \mathrm{F}$ the rates of dissolution converge. 


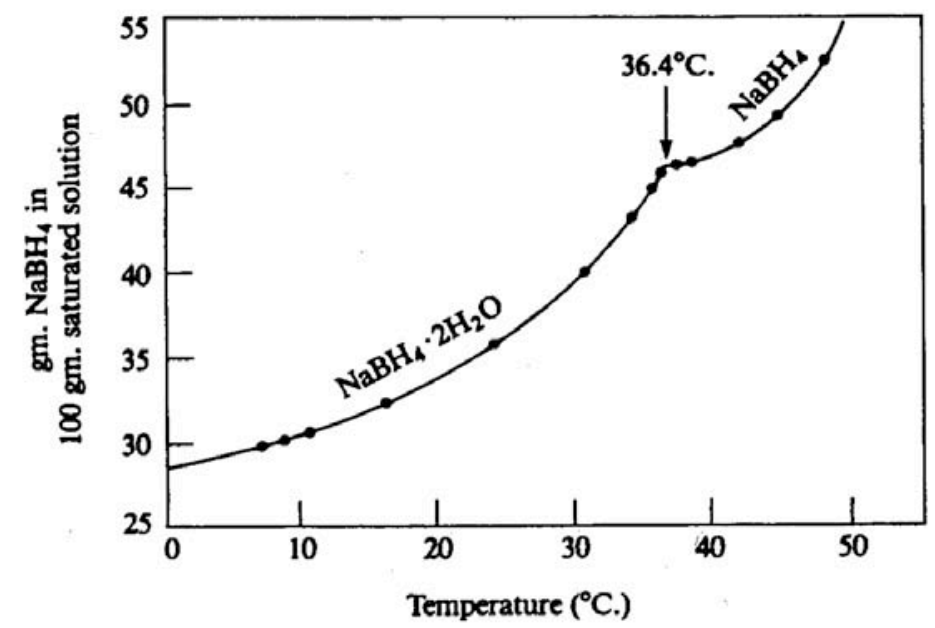

Fig. 61. Solubility of $\mathrm{NaBH}_{4}$ vs temperature. ${ }^{38}$

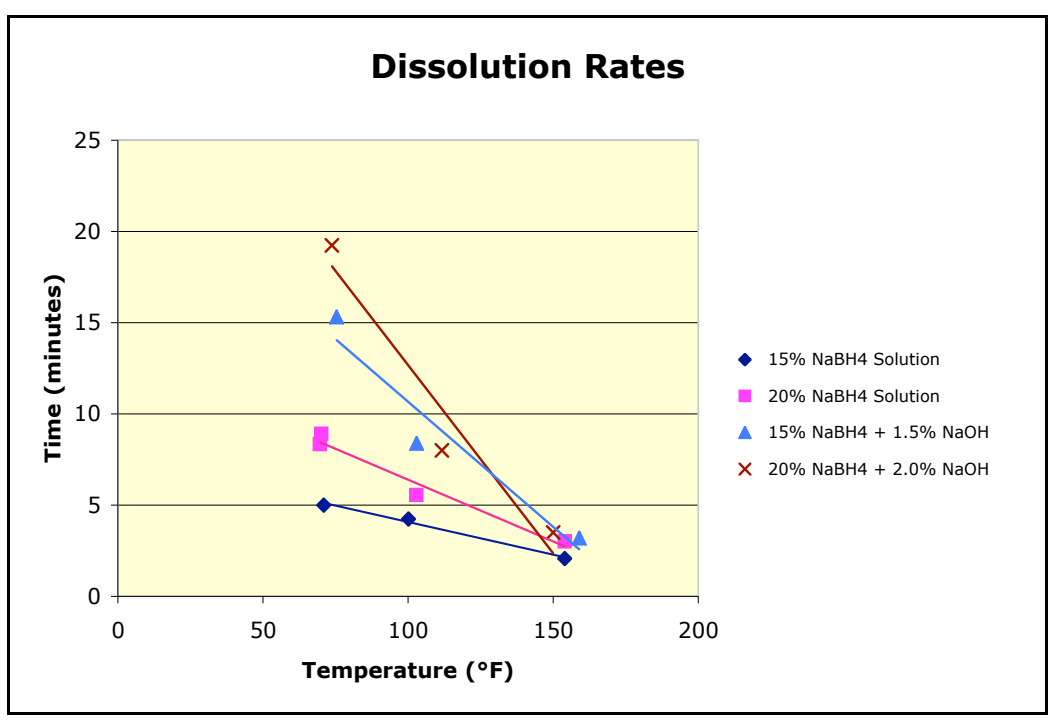

Fig. 62. Dissolution rates of $\mathrm{NaBH}_{4}$.

\section{Reaction Rates}

Scoping tests were performed to evaluate the effects of $\mathrm{Ru}$ catalyst concentration and $\mathrm{NaBH}_{4}$ feed concentration on the rate of hydrogen generation by $\mathrm{NaBH}_{4}$ hydrolysis. The objective of the test was to determine if the rate of hydrogen generation is limited by the availability of hydrolysis sites on the catalyst or by the rate of diffusion of reactant to these reaction sites. The intent was to determine if operating hydrolysis systems using higher catalyst loadings was an effective means of increasing the hydrogen generation rate. The presumption is that, if the rate of hydrogen release is higher at higher catalyst $\mathrm{Ru}$ loadings but is not affected by reactant concentration, the rate of hydrogen generation is limited by the reaction at the catalyst surface. Conversely, if $\mathrm{H}_{2}$ generation increases with increasing reactant concentration in the feed solution but is unaffected by $\mathrm{Ru}$ loading, the rate is controlled by the rate of diffusion of the reactant to the catalyst surface. 
Tests were performed under three sets of conditions. In the first test, the Ru loading on the carbon substrate was $1 \%(\mathrm{w} / \mathrm{w})$ and the $\mathrm{NaBH}_{4}$ concentration in the feed solution was $20 \%(\mathrm{w} / \mathrm{w})$. In the second test, the Ru loading was $2 \%(\mathrm{w} / \mathrm{w})$ and the feed solution $\mathrm{NaBH}_{4}$ concentration was $20 \%(\mathrm{w} / \mathrm{w})$. In the third test, the Ru catalyst loading was $1 \%(\mathrm{w} / \mathrm{w})$ and the $\mathrm{NaBH}_{4}$ concentration in the feed solution was $25 \%(\mathrm{w} / \mathrm{w}) . \mathrm{NaOH}$ was added to the feed solution used in all tests to a concentration of $1 \%(\mathrm{w} / \mathrm{w})$ to suppress uncatalyzed hydrolysis. The system was not preheated, and no attempt was made to control the temperature of the experimental system during testing.

The test configuration used is shown in Fig. 63. In each test the feed solution was added to a stirred vessel containing catalyst chips. In each test the mass of catalyst present in the reactor was $0.5 \mathrm{~g}$. At the start of each test, $20 \mathrm{ml}$ of feed solution were introduced to the reaction chamber through a valved feed tube. The feed tube was valved closed immediately after the solution was delivered. The solution and catalyst were agitated continuously using a magnetically-driven stirring bar. The volume of gas evolved was measured in terms of the volume of water displaced from a tank that was connected to the reactor outlet.

The results of the test are presented in Fig. 64. Under each test condition the rate of gas generation increased after an initially slow rate of evolution. The low initial rate reflects two aspects of the test system. First, an amount of gas had to be generated initially in order to pressurize the system enough for water to be displaced and collected. Second, the rate of the reaction increased after initiation as the temperature of system increased due to the exothermic nature of the reaction.

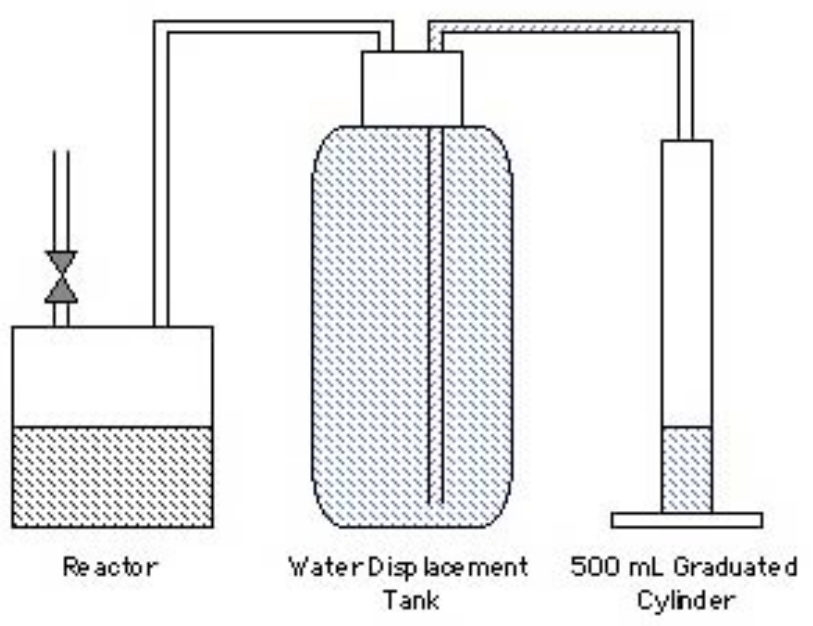

Fig. 63. Experiment configuration.

Significant differences are apparent in the rate of hydrogen generation as a function of catalyst loading. At the $1 \% \mathrm{Ru}, 20 \% \mathrm{NaBH}_{4}$ condition the maximum rate of generation was approximately $1 \mathrm{~L} / \mathrm{min}$, occurring between 13 and 14 minutes after feed introduction. At the $2 \% \mathrm{Ru}, 20 \% \mathrm{NaBH}_{4}$ condition the maximum rate is approximately $2 \mathrm{~L} /$ min, occurring between 10 and 11 minutes after introduction of feed. Other than a slight offset, little difference is apparent in results obtained at different feed concentrations when the catalyst loading was unchanged. 
The results of the test indicate that the hydrolysis rate is affected by the loading of the catalyst. Since the same mass of catalyst was used in all three tests, and since the substrate surface areas should be approximately equal, it is also apparent that the substrate surface is not completely occupied by $\mathrm{Ru}$ at the lower (1\%) loading, and that increased loadings are needed to optimize the reaction rate.

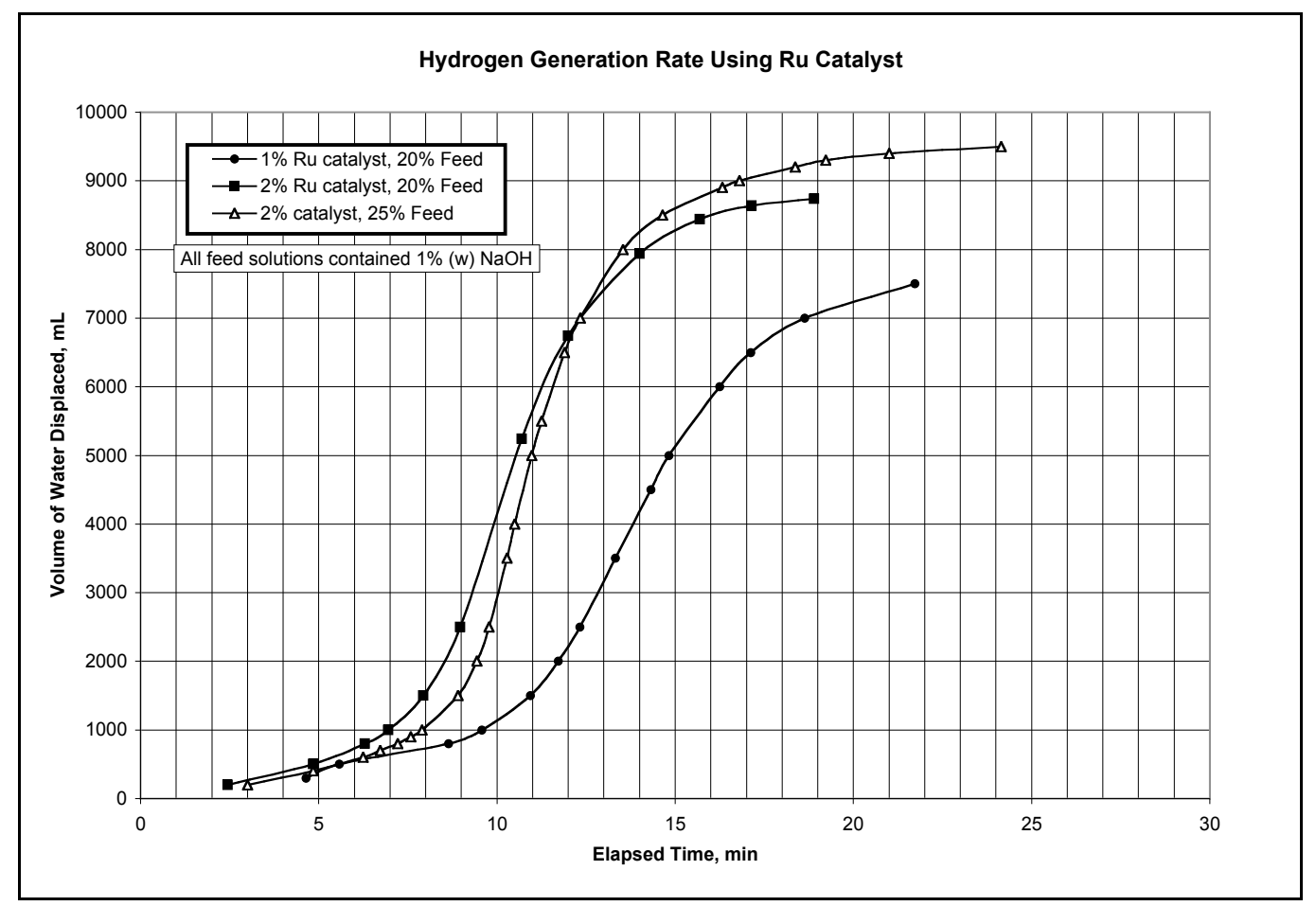

Fig. 64. Hydrogen generation reaction rates using ruthenium catalyst.

\section{Off-Gassing}

Spontaneous hydrolysis of sodium borohydride solution occurs over time, with the rate dependent on concentration, $\mathrm{pH}$, and temperature. The hydrolysis in water causes a rise in the $\mathrm{pH}$, decreasing the rate of decomposition. Typically sodium hydroxide is added to increase the $\mathrm{pH}$ and slow the hydrolysis. The rate of decomposition can be estimated by:

$$
\log _{10} \mathrm{t}_{1 / 2(\min )}=\mathrm{pH}-(0.034 \mathrm{~T}-1.92)
$$

where $\mathrm{t}_{1 / 2}$ is the half-life in minutes and $\mathrm{T}$ is temperature $\left({ }^{\circ} \mathrm{K}\right)$ (Morton International, Inc., 1995).

The dependence of hydrolysis upon various parameters is depicted in the figures below. Figure 65 illustrates the effects of $\mathrm{pH}$ on hydrolysis over time. The higher concentrations of sodium hydroxide slow the spontaneous hydrolysis process. The effect of increased solution temperature is shown in Fig. 66. As can be seen, the increased solution temperature results in increased spontaneous hydrolysis. The effects of temperature and the suppression of the reaction with increased $\mathrm{pH}$ are also illustrated in Fig. 67. 


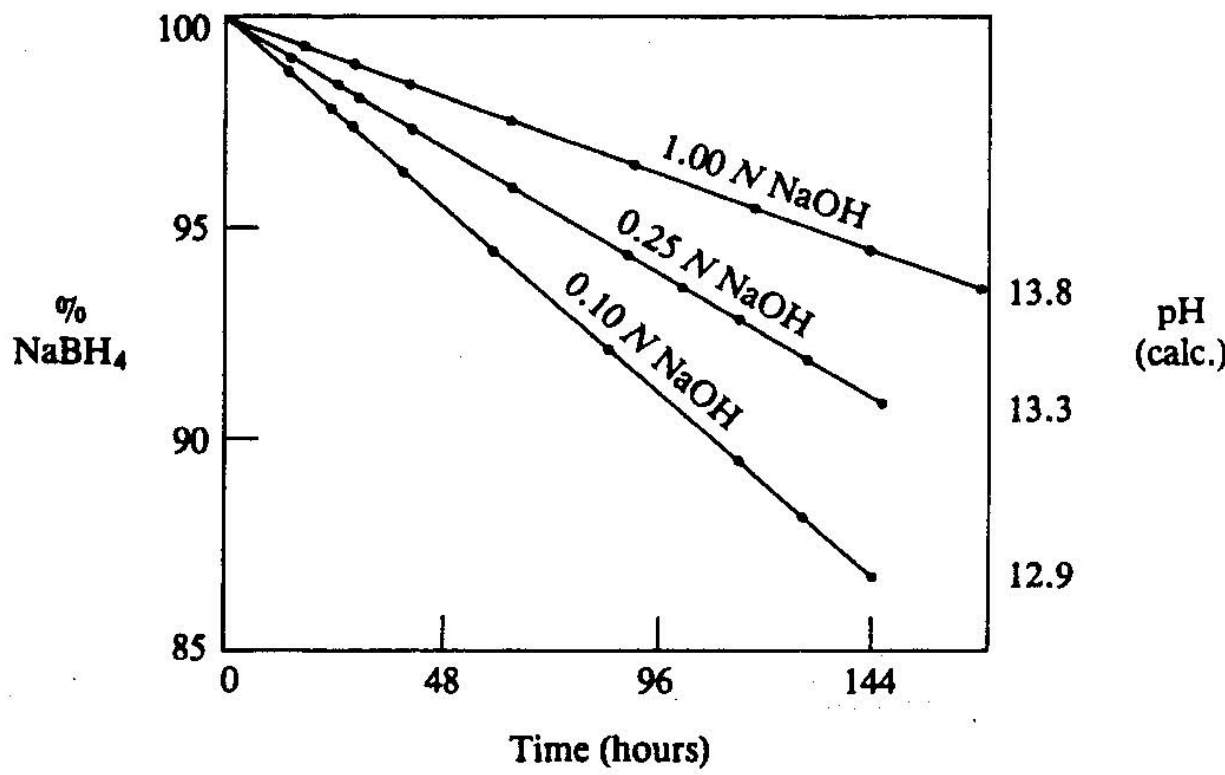

Fig. 65. Stability of $\mathrm{NaBH}_{4} \mathrm{vs} \mathrm{pH}^{38}$

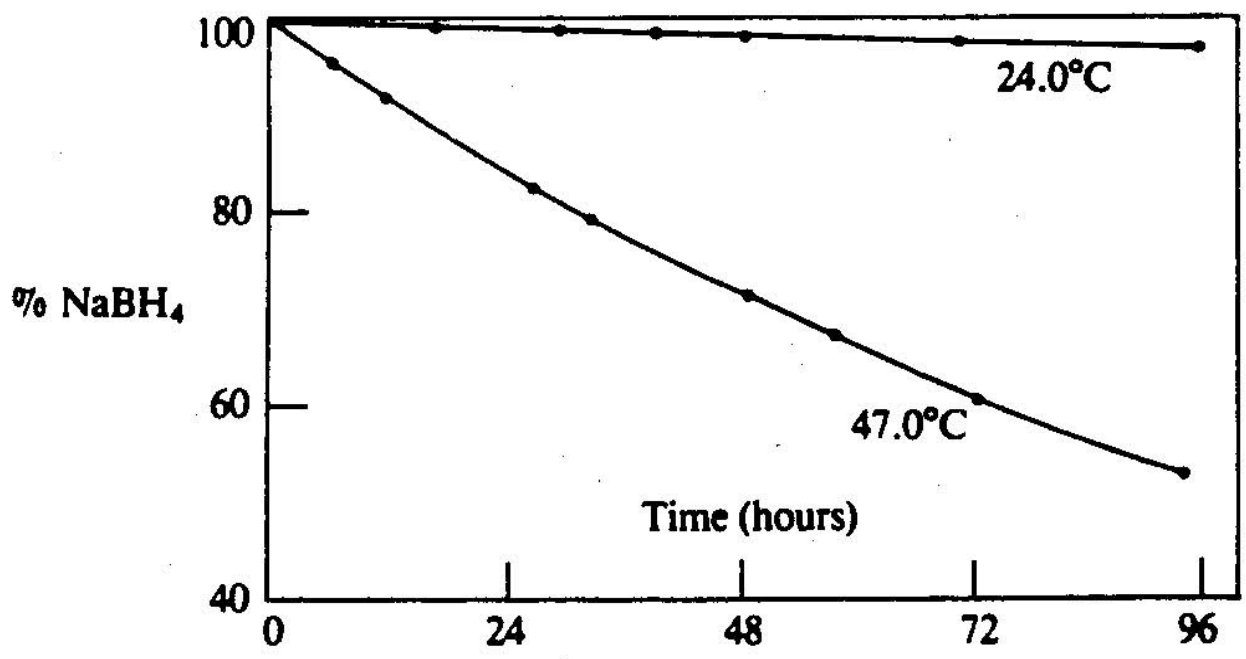

Fig. 66. Stability of $\mathrm{NaBH} 4$ solution vs time at different temperatures. ${ }^{38}$ 


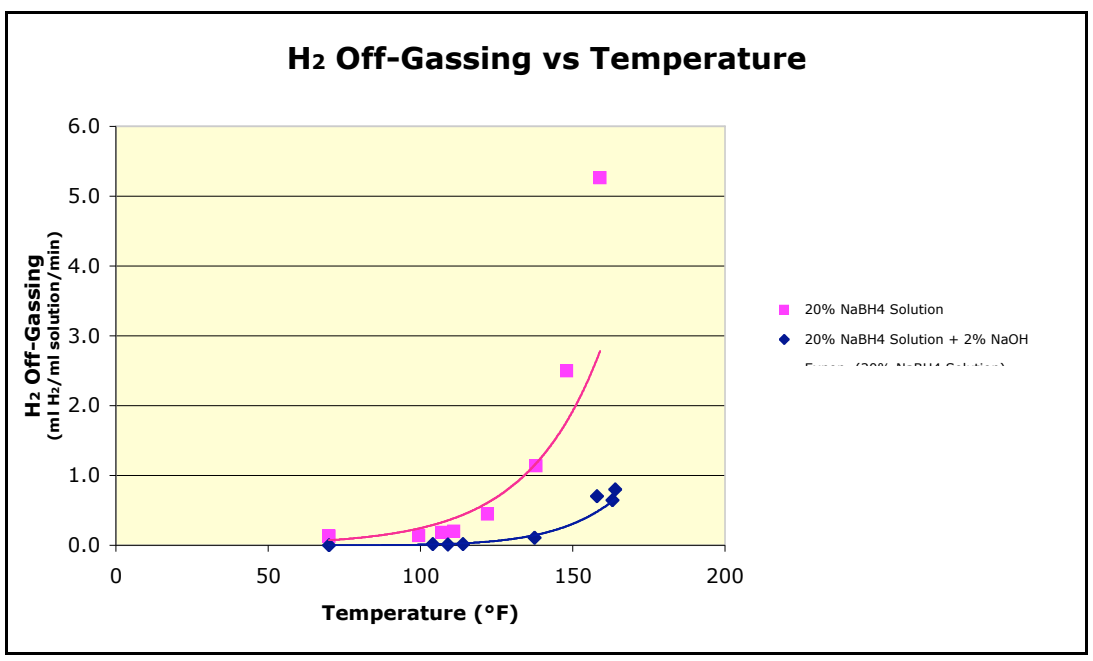

Fig. 67. Hydrogen off-gassing from $\mathrm{NaBH}_{4}$ solution.

How does this spontaneous hydrolysis affect the design of a hydrogen source for a fuel cell? If solution is to be stored for any appreciable period of time, obviously the solution must be stabilized by the addition of sodium hydroxide. However, a system based on water recycling that mixes the solution and consumes it in a relatively short period of time could avoid having to add the sodium hydroxide, thus simplifying the process and potentially enhancing recycling of the reaction by-products.

\section{POWER SOURCE SUMMARY}

This effort successfully demonstrated that a fuel cell hybrid power supply can be a viable option for supplying the power and energy needs of an exoskeleton. In addition, it was shown that the hybrid power supply system is intrinsically safe, and can be operated from hydrogen created on demand by a package of sodium borohydride. The fuel cell was able to deliver power in a pulsed mode, even when operated with an air compressor and hydrogen generated from a chemical hydride.

The next step in refining this system would be to find the lightest, most efficient system components available. There is significant room for size reduction, increased efficiency and simplified operation. The system can be packaged with different form factors.

Fine tuning is needed for the power sharing algorithm and to keep the fuel cell operating at close to full power whenever needed in order to extend the availability of the transient handler. In addition, there is a need for additional work to enhance the stability of the sodium borohydride solution and the pump used to feed the catalytic reactor. In order to increase the specific energy, the design should include a system for fuel cell product water recovery, with the capability to store the sodium borohydride in a dry form and mix it with the product water from the fuel cell, as needed.

A series of experiments were conducted to test the validity of recycling water concept. Tests were conducted to determine the feasibility of recovering water from the fuel cell and the hydrogen generation. Results showed that water recovery is indeed feasible, therefore 
demonstrating the potential to increase the hydrogen storage content from $\sim 4 \mathrm{wt} \%$ up to as high as $21 \mathrm{wt} \%$ (for full water recovery). An initial system configuration has been conceived (Fig. 68).

The hybrid fuel cell system built and tested has potential for many other uses. For example, with slight modifications the control system could be a component for other projects with similar architecture but in a range of power ratings up to $100 \mathrm{~kW}$.

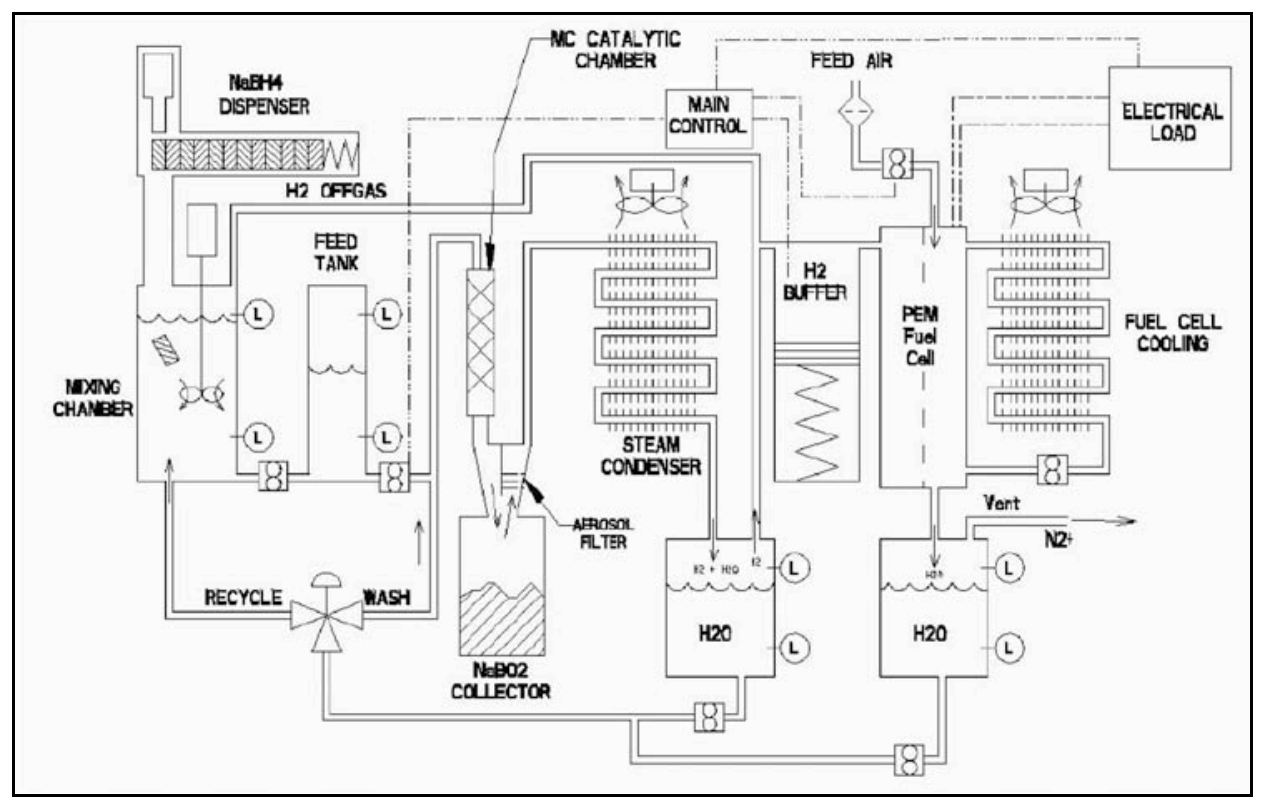

Fig. 68. Hydrogen generation and fuel cell system block diagram (with water recycling). 


\section{ACTUATION}

\section{BACKGROUND ${ }^{\dagger+}$}

As a part of a feasibility study for exoskeleton (Jansen et al., 2000) various types of actuator technologies, subject to their relevance to the exoskeleton project were investigated. A comparison of potential relevant technologies was performed. A designer must make decisions based on critical design parameters such as (1) power/mass, (2) power/volume, (3) stress, (4) strain, (5) steady-state efficiency, (6) power expended during load holding, (7) bandwidth or tracking performance, (8) auxiliary transmission system, (9) auxiliary power modulation equipment, and (10) ease of controllability. Depending on the type of actuator, some of the critical design parameters will not be applicable or not available due to design specificity. Actuator technologies can be broken down into two major groups: conventional and nonconventional. Conventional actuators are electric motors, hydraulic, and pneumatic. The nonconventional actuators are the piezoelectric and magnetorestrictive. While others have examined these types of actuators in the past, their relevance to the exoskeleton project is the main consideration here.

Of the conventional actuators (Table 5), it appears that hydraulics is the best candidate for an exoskeleton application. The power-to-weight and power-to-volume ratios of hydraulics typically exceed the ratios for electromagnetic actuation by factors of 10 and 5 respectively. The closed-loop bandwidth of pneumatics is typically 5 to 6 times less than that for hydraulics, thus limiting the ability to track at $2 \mathrm{~Hz}$. Based on packaging and the ability to track the human, hydraulics is the preferred conventional actuator.

Table 5. Comparison of conventional actuators

\begin{tabular}{|c|c|c|c|}
\hline Item & Electromagnetic Actuators & Hydraulic Actuators & Pneumatic Actuators \\
\hline Bandwidth & $\begin{array}{l}\text { Transmission required. Motors } \\
\text { sized } 10 \text { to } 20 \% \text { larger due to } \\
\text { transmission efficiencies. }\end{array}$ & $\begin{array}{l}\text { High performance servos in the } \\
50 \text { to } 100 \mathrm{~Hz} \text { range. Dependent } \\
\text { on actuator and controller } \\
\text { design. }\end{array}$ & 5 to $6 \mathrm{x}$ less than hydraulics. \\
\hline $\begin{array}{l}\text { Power to } \\
\text { Weight }\end{array}$ & $\begin{array}{l}\text { Approximately } 1 / 10 \text { hydraulics } \\
\text { ratio. }\end{array}$ & Excellent. & $\begin{array}{l}\text { Elastic type actuators are } \\
\text { similar to hydraulics. Hard- } \\
\text { shell type actuators are similar } \\
\text { to electric actuators. }\end{array}$ \\
\hline $\begin{array}{l}\text { Power to } \\
\text { Volume }\end{array}$ & $\begin{array}{l}\text { Approximately } 1 / 5 \text { hydraulics } \\
\text { ratio. }\end{array}$ & Excellent. & $\begin{array}{l}\text { Elastic type actuators are } \\
\text { similar to hydraulics. Hard- } \\
\text { shell type actuators are similar } \\
\text { to electric actuators. }\end{array}$ \\
\hline Packaging & $\begin{array}{l}\text { Transmission increases vol by } \\
20-50 \% \text {. Power electronics can } \\
\text { exceed size of actuator. }\end{array}$ & $\begin{array}{l}\text { No transmission. Power } \\
\text { electronics are inconsequential. } \\
\text { Sound proofing pumps } \\
\text { difficult. }\end{array}$ & Similar to hydraulics. \\
\hline $\begin{array}{l}\text { Load } \\
\text { Holding }\end{array}$ & $5-10 \%$ or rated power required. & $\begin{array}{l}\text { No power for linear actuators } \\
\text { with asymetrical cylinders. } 1 \text { to } \\
10 \% \text { for symetrical cylinders } \\
\text { and rotary actuators. }\end{array}$ & Similar to hydraulics. \\
\hline
\end{tabular}

\footnotetext{
it Appendix F provides additional background details regarding the basis of the approach taken (Jansen et al., 2000).
} 
However, there are issues concerning the use of hydraulics, which must be taken into account. First, the power loss at the servo valves associated with flow control is significant and has a serious impact on the overall power supply sizing. Second, noise is a serious problem associated with hydraulics that can be difficult to mitigate due to the large acoustical wavelengths.

While none of the nonconventional materials have reached the level of maturity achieved with hydraulics, both the piezoelectric and the magnetostrictive materials hold great promise and should be investigated further. In particular, a piezoelectric pump was shown to have the potential for power densities approaching those of hydraulics, as well as high steady-state efficiency, and no wasted power holding a load. The magnetostrictive actuators are similar to piezoelectric actuators, but with lower anticipated efficiencies and load holding capability similar to that of electric motors. Power electronics is an issue for both that is the subject of ongoing research.

At the start of the exoskeleton program, two approaches were proposed by ORNL to address the compact actuator needs of this initiative that could meet the requirements of receiving electrical energy and convert it to mechanical energy through a hydraulic fluid medium. The first was a high-risk, high-payoff path based on utilizing piezoelectric materials as the primary pumping source to control the motion of the associated hydraulic actuators. The second approach was a less risky, but with lower potential performance, based on utilizing high-speed electric motors turning a miniature hydraulic pump which in turn feeds conventional hydraulic actuators. Work on the piezoelectric based actuators will be the main focus of this section; however, at the end of this section there will be a discussion of the progress of the second approach.

\section{PIEZOELECTRIC ACTUATOR APPROACH}

Piezoelectric material can convert electrical energy to hydraulic energy and act as a flow control device similar to a hydrostatic pump. Further, the power-to-weight and power-to-volume ratios of this device can be shown to be potentially superior to current methods of placing an electric motor in tandem with a hydraulic pump in tandem with a flow control valve. Presently, lead zirconate titanate (PZT) ceramics are the materials of choice for piezoelectric ceramic actuators. These materials have piezoelectric coefficients $\mathrm{d}_{33}$, around 200 to $750 \square 10^{-12} \mathrm{~m} / \mathrm{V}$, and strain levels from 0.05 to $0.1 \%$. However, there is a new family of piezoelectric material that has the potential to change future piezoelectric actuators. One type, a single crystal form of relaxorbased ferroelectric materials, lead zirconate niobate / lead titanate (PZN-PT), has been observed to have piezoelectric coefficients $\mathrm{d}_{33}$, around $2000 \times 10^{-12} \mathrm{~m} / \mathrm{V}$, and strain levels from $0.5 \%$ to $1.0 \%$, an order of magnitude greater than conventional PZT material. While these materials have been known for several years, their potential for high performance actuators has only recently been recognized. Large forces and small displacements occur in a piezoelectric crystal when an electric field is applied and is the major limitation for their utilization. Compressive stress levels can be as high as $35 \mathrm{MPa}$ (5000 psi), whereas the tensile stress levels can be only 5 to $10 \%$ of the compressive stress level. Typically, a mechanical force bias is required to avoid the tensile stress limits. Displacements are typically very small for piezoelectric materials. One difficulty of using piezoelectric material pertains to converting small displacements to large motion (i.e., the

transmission problem). Various approaches include the "inch-worm," where micro-stepping 
action through rapid lock-and-move sort of motion are used to create a large linear motion. Another concept is that of a piezoelectric hydraulic pump, where small quantities of fluid move at very high frequencies. The accumulation of small drops of fluid at a high rate adds up to a large flow rate. Both of these concepts are based on the idea of the cyclic motion of the piezoelectric material. This latter approach of moving one drop of fluid at a time is the one being taken in this project and has the potential of achieving the radical improvement in motion control for a high-power density actuator. It can be shown that the maximum power per volume of the piezoelectric actuator is directly proportional to the stack operating frequency. At a $1 \mathrm{kHz}$ rate, the power density is approximately $2.7 \mathrm{~J} / \mathrm{kg}$ per cycle for a single-crystal piezoelectric material, lead zirconate niobate $(\mathrm{PZN})$. Larger energy-storage terms have been reported in the literature, but because of fatigue life limitations (recall that due to the transmission problem, billions of cycles will be required from this material), this reduced value is more reasonable. Power-to-volume for electric motors around the $1 \mathrm{~kW}$ level is approximately $1.3 \mathrm{~W} / \mathrm{cm}^{3}$. To obtain a factor of 5 times this value and therefore rival that of hydraulic actuators, the cyclic frequency would have to be around $1 \mathrm{kHz}$. While the power-to-weight for an electric motor at the $1 \mathrm{~kW}$ level is approximately $300 \mathrm{~W} / \mathrm{kg}$, the single crystal material would have a power-toweight about 3 times this value. If one takes into consideration that electric motors are high speed and low torque devices and that some sort of transmission system (which could add up to $50 \%$ of additional weight) would be required to connect an electric motor to a hydraulic pump, the power-to-weight value for the piezoelectric materials as compared to an electric motor could easily be 5 times larger.

To attack this problem, two approaches were taken concerning the transmission problem. These two approaches are predominately associated with how the valving of the pumping chamber should be accomplished. While there were other technical concerns pertaining to stack voltage and fluid modulation methodology, valving was the primary demarcation of the two approaches. The first approach was to use very responsive passive valves that allowed fluid into and out of the chamber without significant losses. The second approach was to utilize active valves for the pumping chamber based on piezoelectric actuators. The ORNL focused on the first approach with passive valving and Virginia Polytechnic Institute (VPI) focused on the second approach based on active valving. The target objective, based on early estimates pertaining to the power requirements, was $600 \mathrm{~W}$ of mechanical power at an operating pressure of either 1,000 to 2,000 psi.

\section{Passive Valve}

ORNL has developed a novel hydraulic pump/actuator test bed (Fig. 69), building upon the central idea of a distributive passive valving (Fig. 70) scheme to achieve very high frequencies in moving small drops of fluid rapidly in and out of the pumping chamber.

Due to power electronics limitation, the operational frequency of the stack could not exceed $400 \mathrm{~Hz}$. For the final system, frequencies around $1 \mathrm{kHz}$ are required. The following results, which benchmark the performance of ORNL's piezoelectric pump, are linearly scaled to evaluate the overall performance potential of the pump. The first measurement is for no load flow test, where the test fluid is pumped into a fixed volume for 100 seconds. The theoretical and the measured flows are shown in Fig. 71. Approximately 60\% volumetric efficiency at frequencies below $300 \mathrm{~Hz}$ was achieved. It is apparent from Fig. 71 that significant leakage flows still exist. 
Ball check valves on the piston side have significantly reduced the leakage flows from the previous design; however, they have not been placed on the cylinder side of the pump (i.e., the outlet valve) due to required modifications of the pumping chamber to support this change. At higher frequencies (around $300 \mathrm{~Hz}$ ), the results (Fig. 71) suggests that the flappers are not responsive enough. Next, the deadhead pressure was tested by blocking the piston outlet flow. Theoretical pressures should exceed 1000 psi over a much larger frequency band than those shown in Fig. 72 (based on the assumed structural compliance and fluid bulk modulus values).

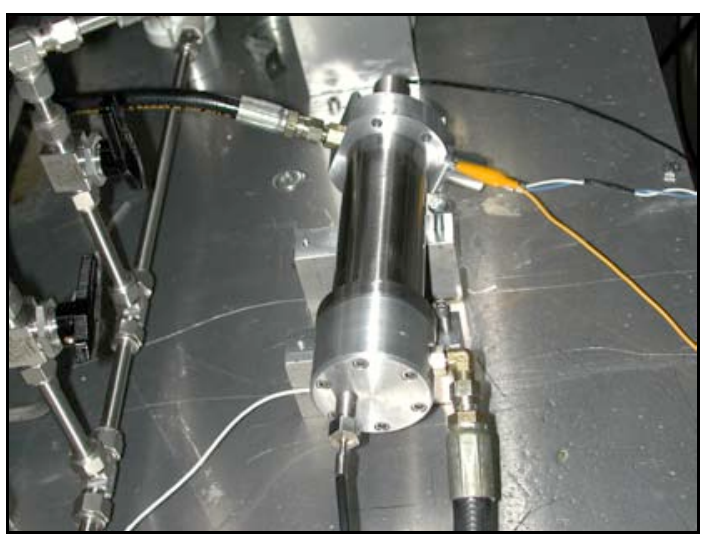

Fig. 69. ORNL's piezoelectric pump.

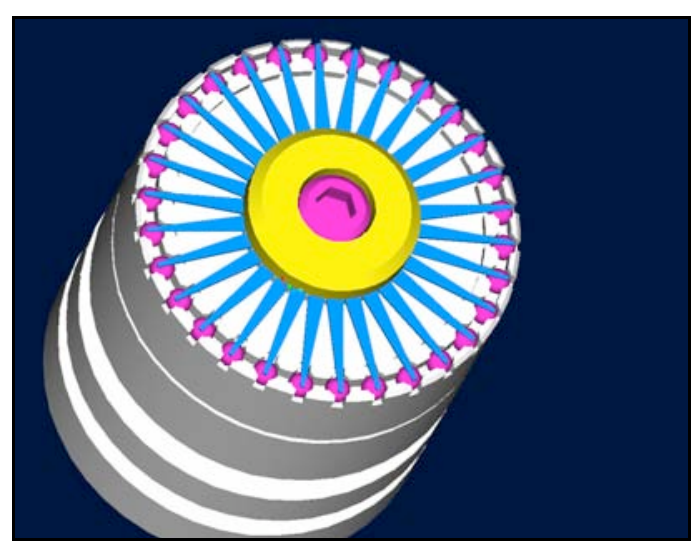

Fig. 70. Distributive valves.

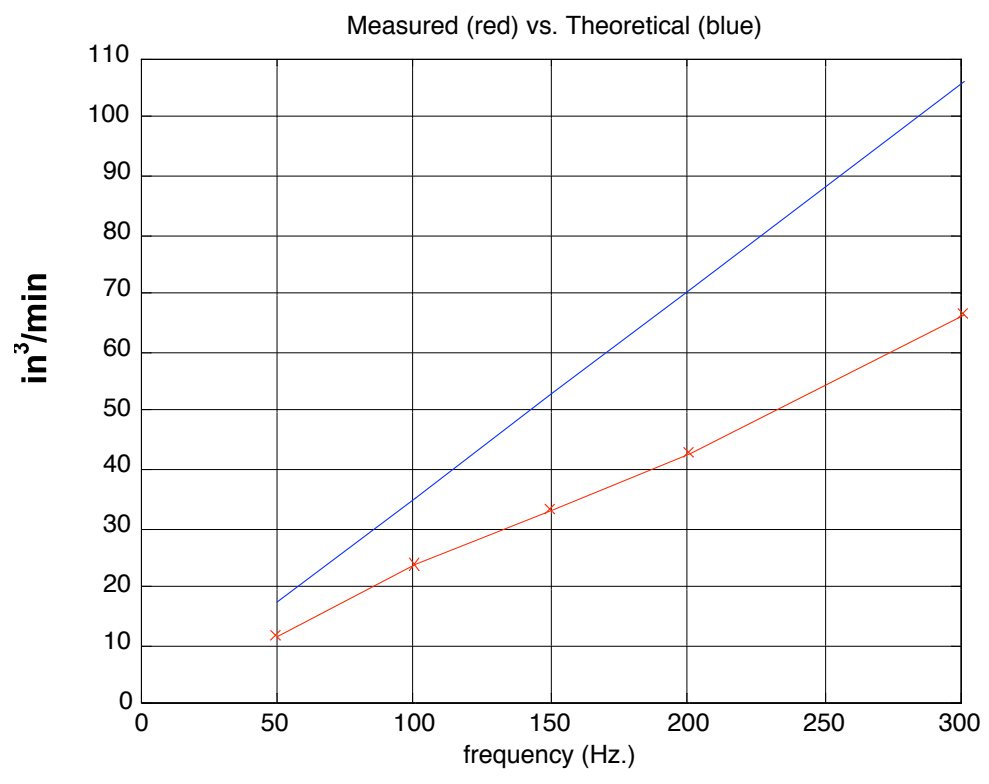

Fig. 71. ORNL piezoelectric pump flow. 


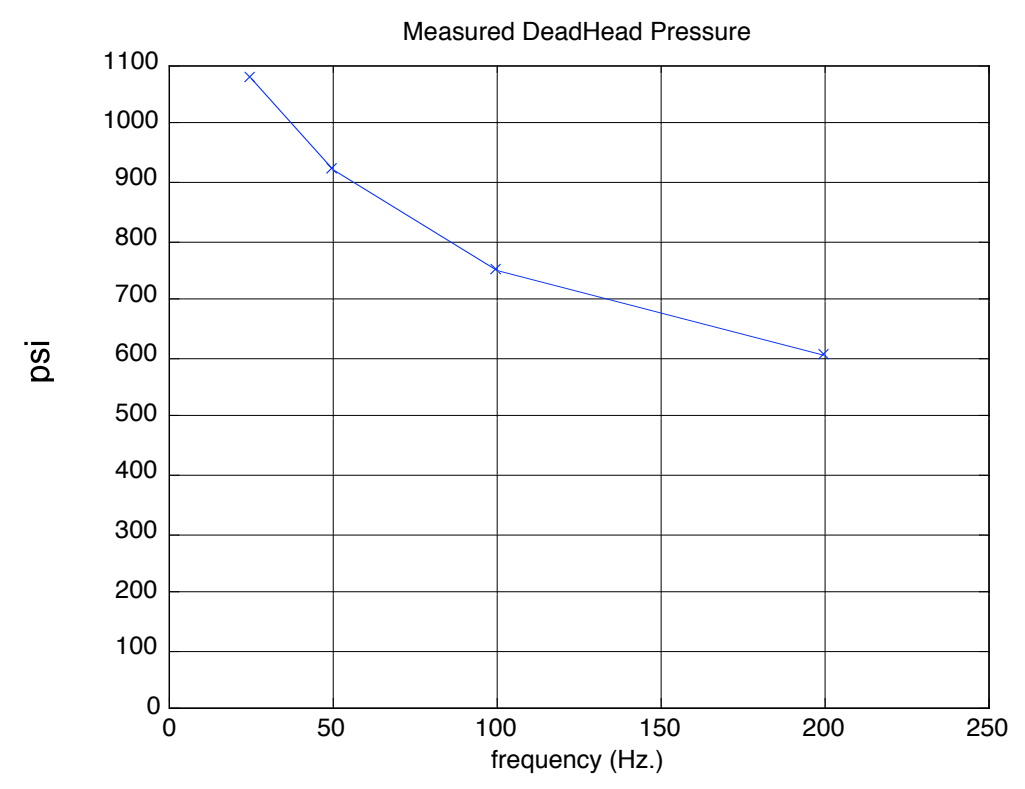

Fig. 72. Deadhead pressure versus stack frequency.

There are two possible explanations for the discrepancies with the theoretical values: leakage and air that has not been properly evacuated or entrained into the working fluid. Again, the ball check valves that we would like to install on the cylinder should eliminate the leakage problem. Finally, the power produced by our pump should be around $200 \mathrm{~W}$ at $1 \mathrm{kHz}$ and should scale linearly at lower frequencies. At $300 \mathrm{~Hz}$, the power should be around $67 \mathrm{~W}$. Figure 73 shows the results of a power test we have performed at various stack operating frequencies. This test entailed having the pump move fluid against a constant back pressure. As demonstrated in this figure, we are roughly off by a factor of 4 from the expected value (i.e., roughly $14 \mathrm{~W}$ at $300 \mathrm{~Hz}$ compared with the theoretical expected $67 \mathrm{~W}$ ).

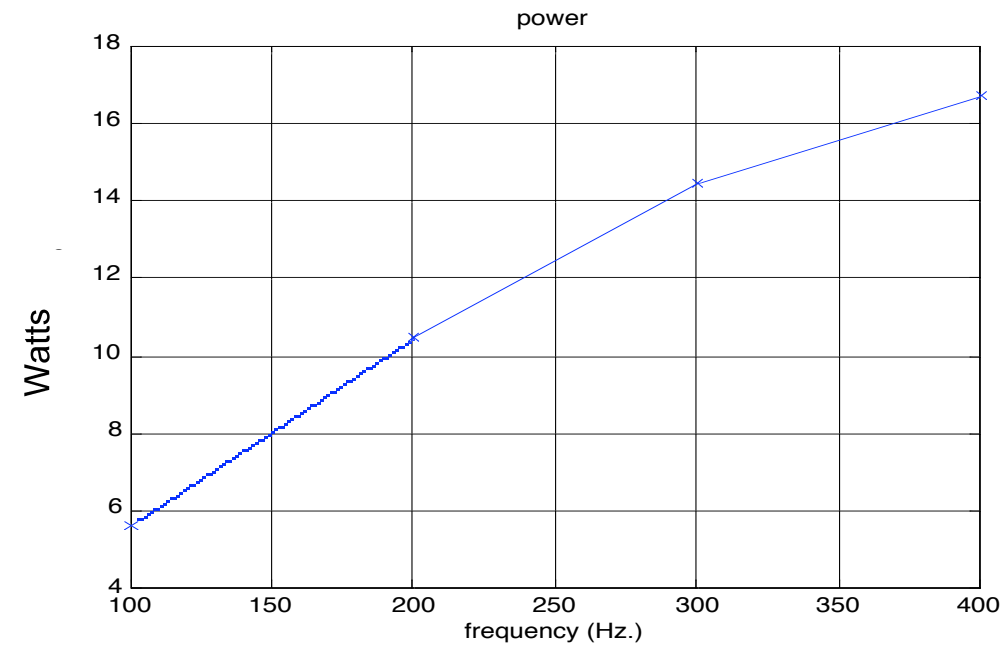

Fig. 73. Power out of the piezoelectric pump. 


\section{Active Valve}

VPI successfully designed, built, and tested a piezo-hydraulic actuator that achieved $3.6 \mathrm{~W}$ of mechanical power out. Figures 74 and 75 show the major components and the overall system integration. Further, they developed the system-level models that accurately predicted the performance of the device. Presently, the major system-level limitations were: high current driver requirements for operation in the $\mathrm{kHz}$ range which resulted in large drive electronics and viscous losses in the hydraulic circuit and valves limited operating speed of the pump.

Figure 76 shows how velocity varies with both loading and frequency. Studies have demonstrated that the output power was highly dependent on the valve phasing. Power variations of $15-20 \%$ were noted by varying the valve phasing about the nominal conditions as can be seen in Fig. 77.

Cylinder velocities of $39 \mathrm{~mm} / \mathrm{sec}$ were measured under no-load conditions (Fig. 76). Velocities of $10 \mathrm{~mm} / \mathrm{sec}$ were measured at loads of $22 \mathrm{~kg}$. Maximum output power of $3.6 \mathrm{~W}$ was measured at an optimal operating frequency of $140 \mathrm{~Hz}$ (see Fig. 77).

For the VPI actuator, the primary limitations to increasing the power output were: viscous losses in the hydraulic circuit limited flow at higher frequencies and decreased power output at frequencies higher than about $140 \mathrm{~Hz}$ and kilohertz frequency operation necessitated high current output power supplies and these power supplies proved to be difficult to miniaturize.
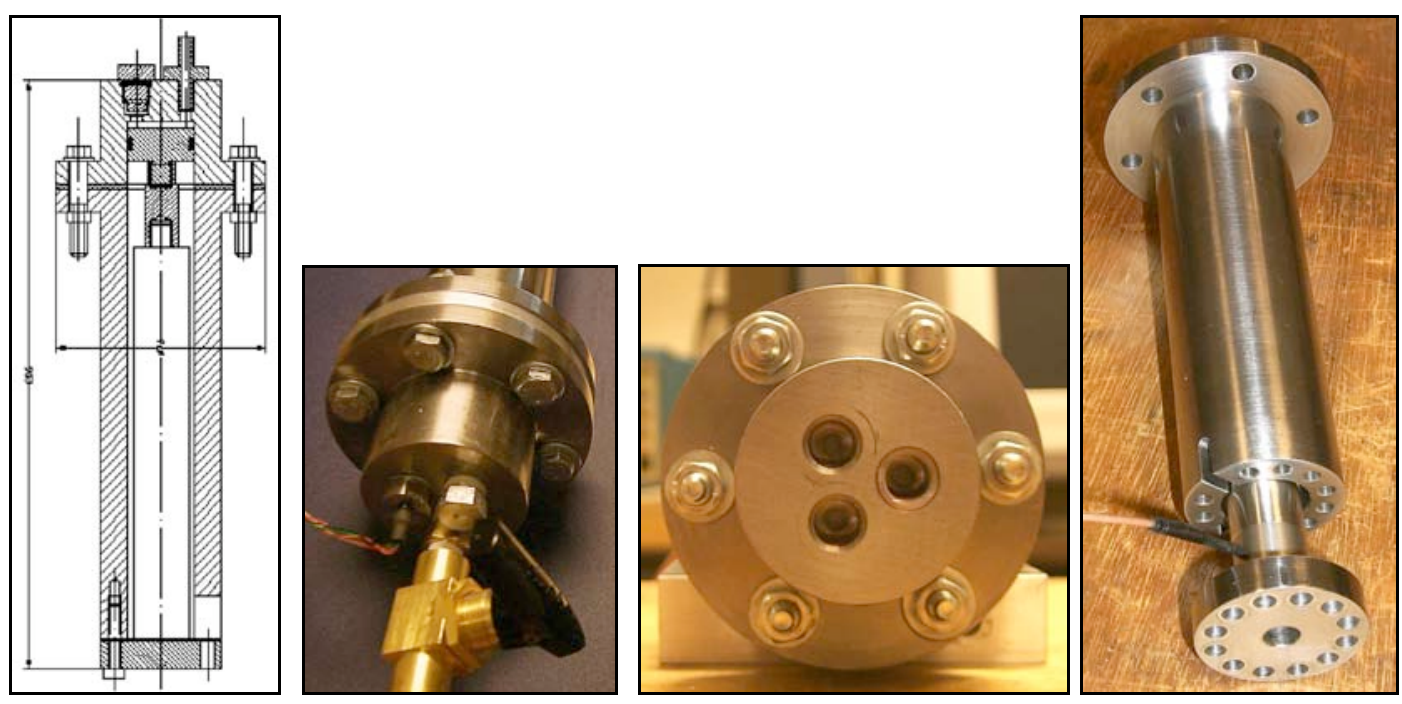

Fig. 74. VPI pump.

(Assembled drawing, assembled pump, chamber outputs, casing.) 


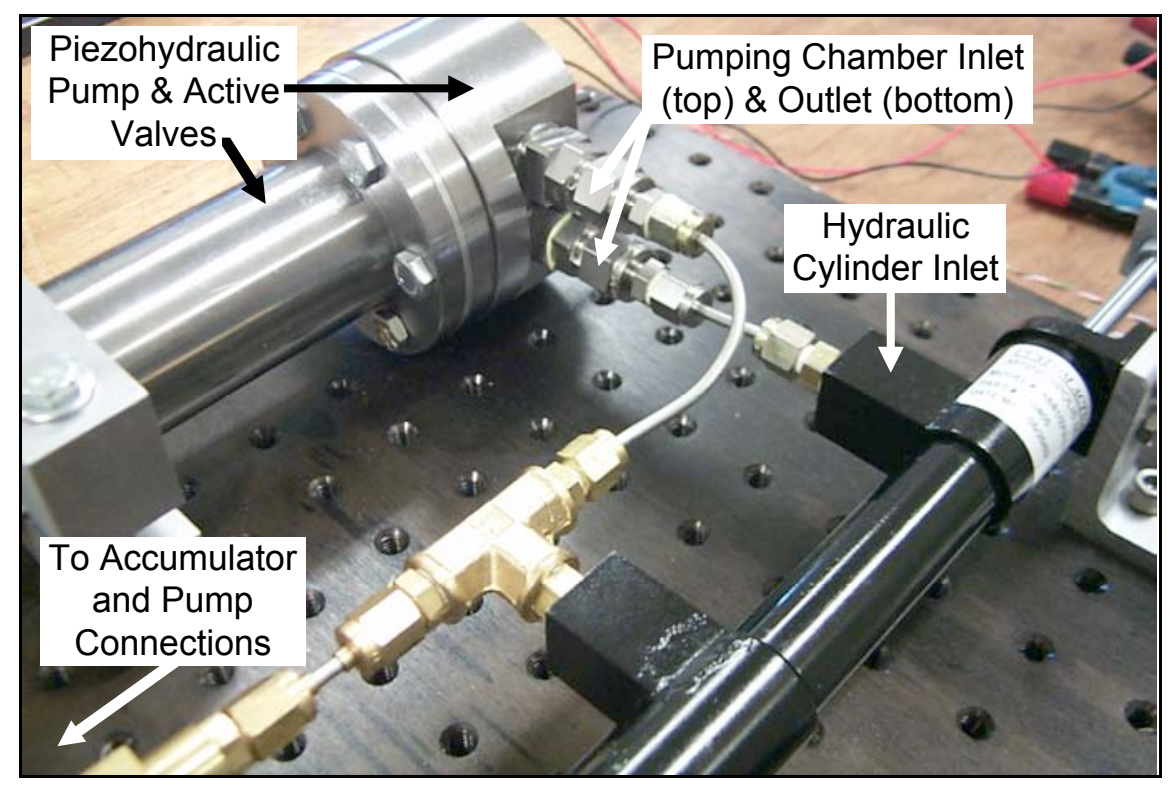

Fig. 75. Integrated system.

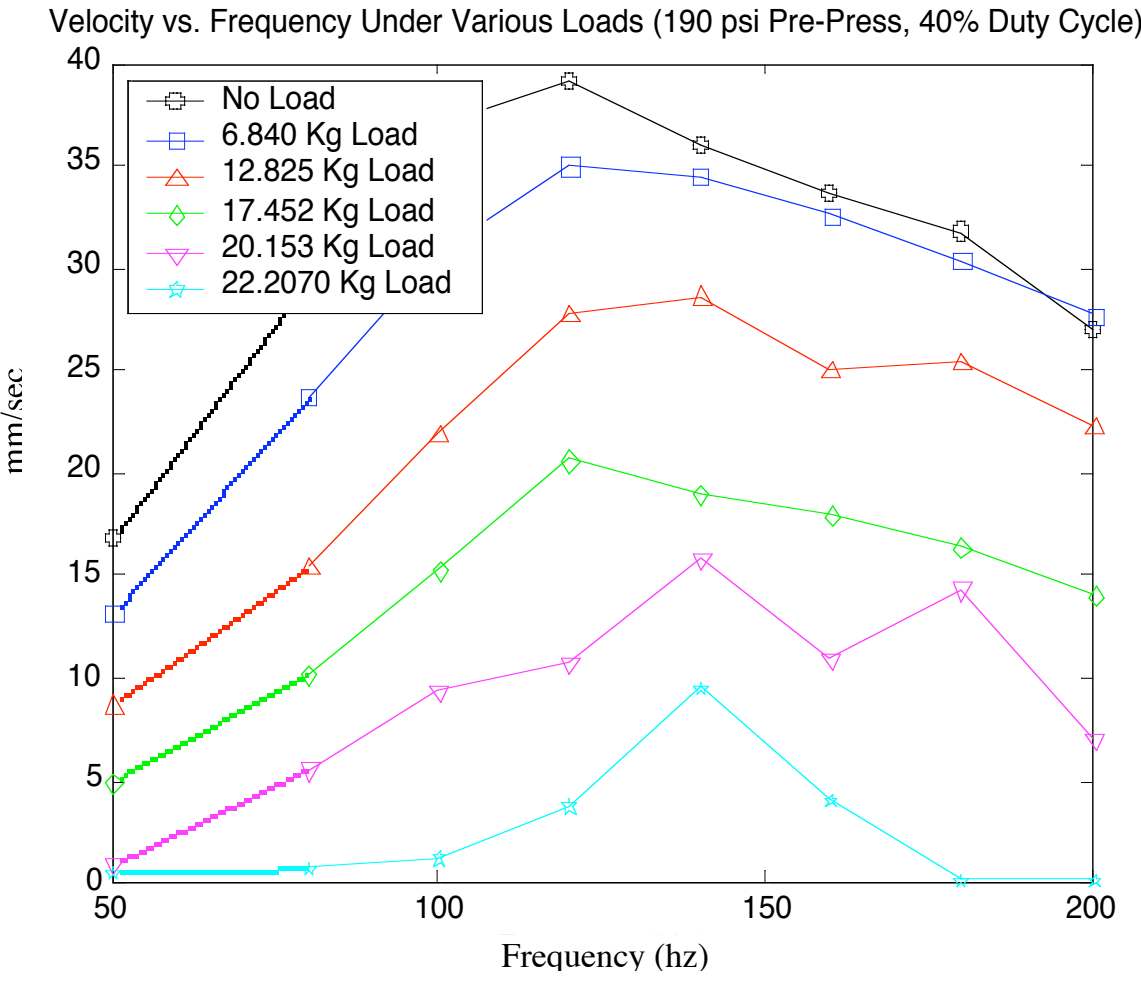

Fig. 76. Velocity vs frequency under various loads. 


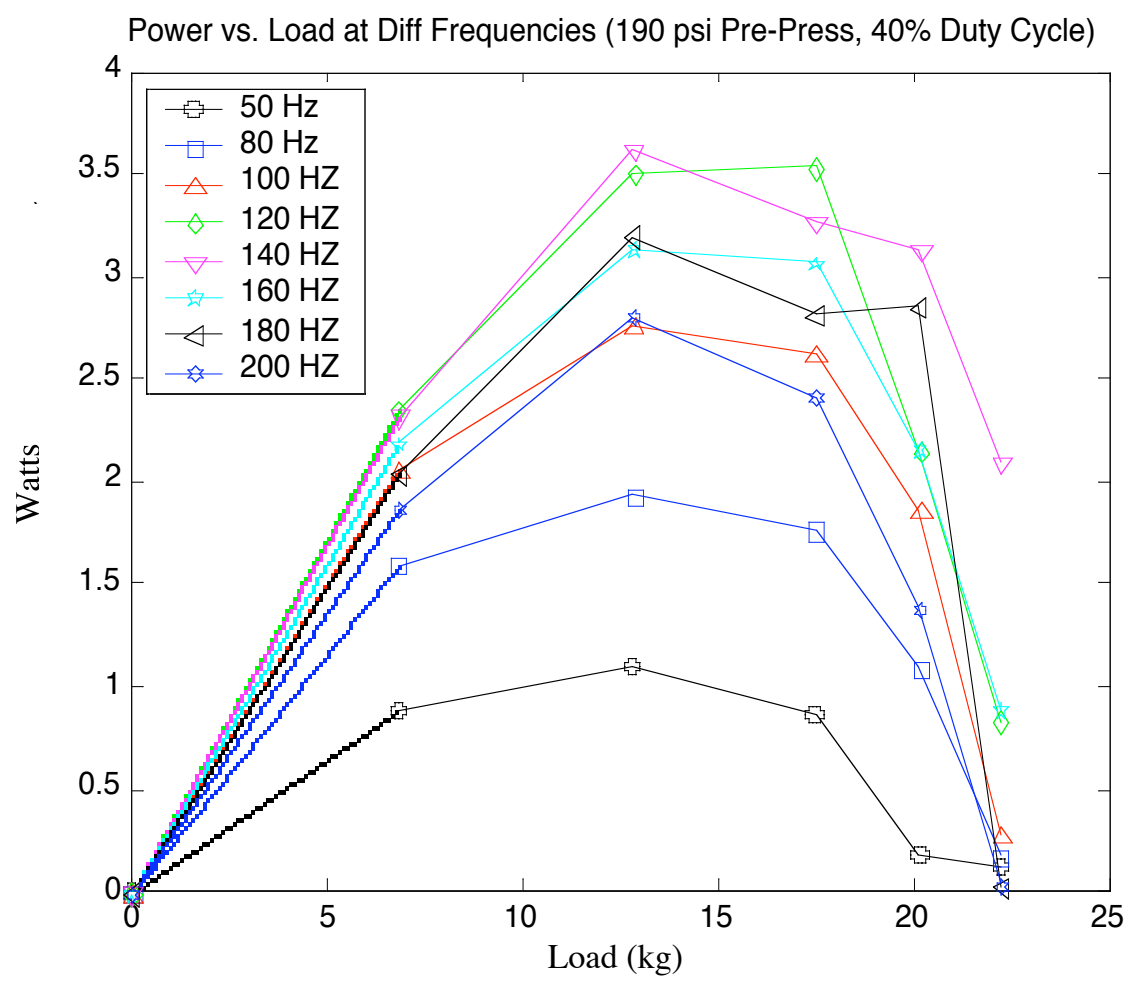

Fig. 77. Power vs load at different frequencies.

\section{Conclusion of Piezoelectric Actuator Initiative}

High power densities are theoretically possible only if the piezoelectric stack is driven at high frequencies since the power density is directly proportional to stack frequency. While this approach holds great promise, this technology is currently not going to be available to meet the exoskeleton program needs. A second approach based on a high-performance, high-speed electric motor turning a miniature hydraulic pump will be examined next.

\section{ELECTRIC MOTOR APPROACH}

As was previously discussed, ORNL has been investigating two approaches for actuation. The first was a piezoelectric pump scheme that would feed a hydraulic actuator. The second was a high-speed electric motor scheme that drives a miniature hydraulic pump. The piezoelectric pump scheme was based on driving a piezoelectric stack at high frequency allowing fine control of hydraulic fluid (basically a drop at a time). In comparison, the highspeed electric motor/pump is analogically similar to the piezoelectric pump. For the high-speed electric motor/pump, the electric motor turns a miniature hydraulic pump at a high angular velocity allowing fine control of hydraulic fluid. Functionally, the electric motor exerts a very low moment but has a very large displacement. High power densities are possible (and have 
been tested) since the electric motor rotor volume is directly proportional to the torque generated. Therefore, power density is roughly proportional to motor speed. For the near term, the highspeed electric motor/pump appears to be most promising and will allow the efficient conversion of electric energy to hydraulic energy.

Without cooling, a frameless brushless electric motor will have a power-to-weight around $300 \mathrm{~W} / \mathrm{kg}$. With a well-designed cooling system exploiting the hydraulic fluid as the cooling medium in a heat exchanger, it could be possible to extend the power density up to 3 times these values. With a carefully selected hydraulic pump that is matched to the speed and torque characteristic of the electric motor, a transmission system would be avoided. Preliminary calculations indicate that a small hydraulic pump with 1-2 cc/rev of displacements with high volumetric efficiency would be required. Both the electric motor and the hydraulic pump will be placed inside the reservoir to reduce the overall sound levels and facilitate heat transfer.

Lastly, we will not utilize standard sizing practice for flow control valves (i.e., servo-valves). For hydraulic actuation, servo-valves control the flow by means of large pressure drops across internal orifices, which is analogous to a resistive load for electrical systems. It is not uncommon, due to the size of the servo valves, for up to $33 \%$ of the total power transmitted to the load to be lost in the form of heat across these servo valves. Even higher losses are possible in certain loading/flow scenarios. To mitigate losses of the servo valves, servo valves will be rated to operate at significantly lower pressure drops.

To demonstrate the overall size of the components, a computer simulation of ORNL's exoskeleton system was modeled and the hydraulic flows and pressure needs were ascertained for a fast walking scenario of $2.2 \mathrm{~m} / \mathrm{sec}$ carrying a combined load of $300 \mathrm{lb}$. The requirements for the hydraulic pump are shown in Figs. 78 to 80. Based on prolonged discussions with a leading aerospace company (Moog, Inc.), the required hydraulic pump can be built under $2 \mathrm{lb}$ and the electric motor to drive it would be under $4 \mathrm{lb}$. With an additional $1.5 \mathrm{lb}$ for auxiliary pumping the total weight would be around $7.5 \mathrm{lb}$.

In summary, by means of operating the electric motor at very high rpm, cooling the electric motor, and allowing lower pressure drop across the fluid control valves, an efficient electricallydriven hydraulic pump can be designed that will both save energy ( $>30 \%)$ and improve power density compared to a conventional constant pressure system. It appears that high-end electric motors, in conjunction with very specialized hydraulic pumps that are now available from industry make it feasible to effectively convert electrical energy from a fuel cell to hydraulic energy. 


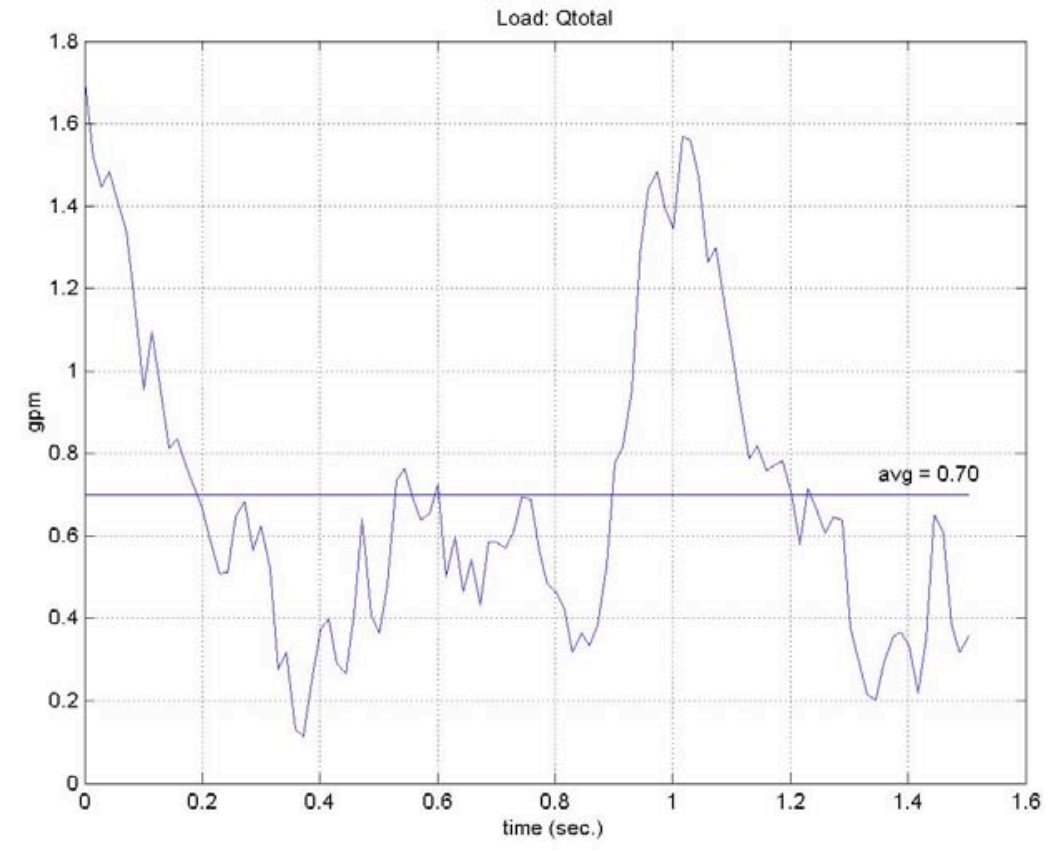

Fig. 78. Flow requirement of pump.

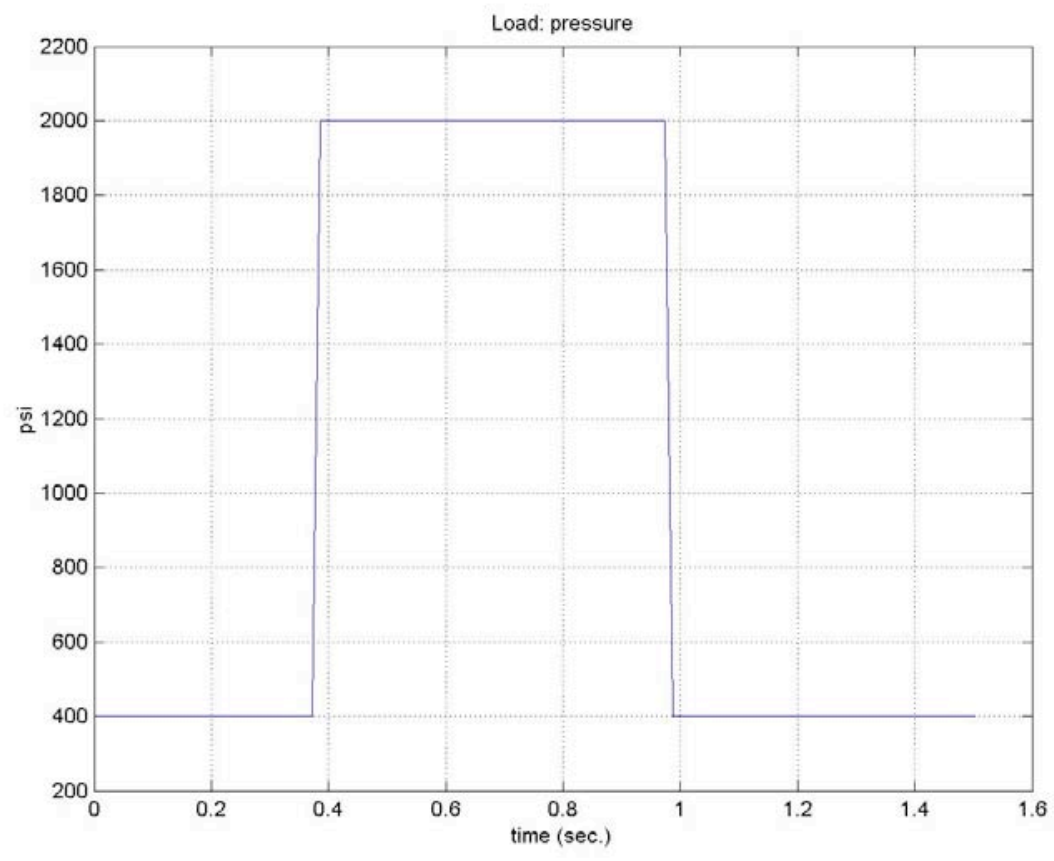

Fig. 79. Pressure requirement of pump. 


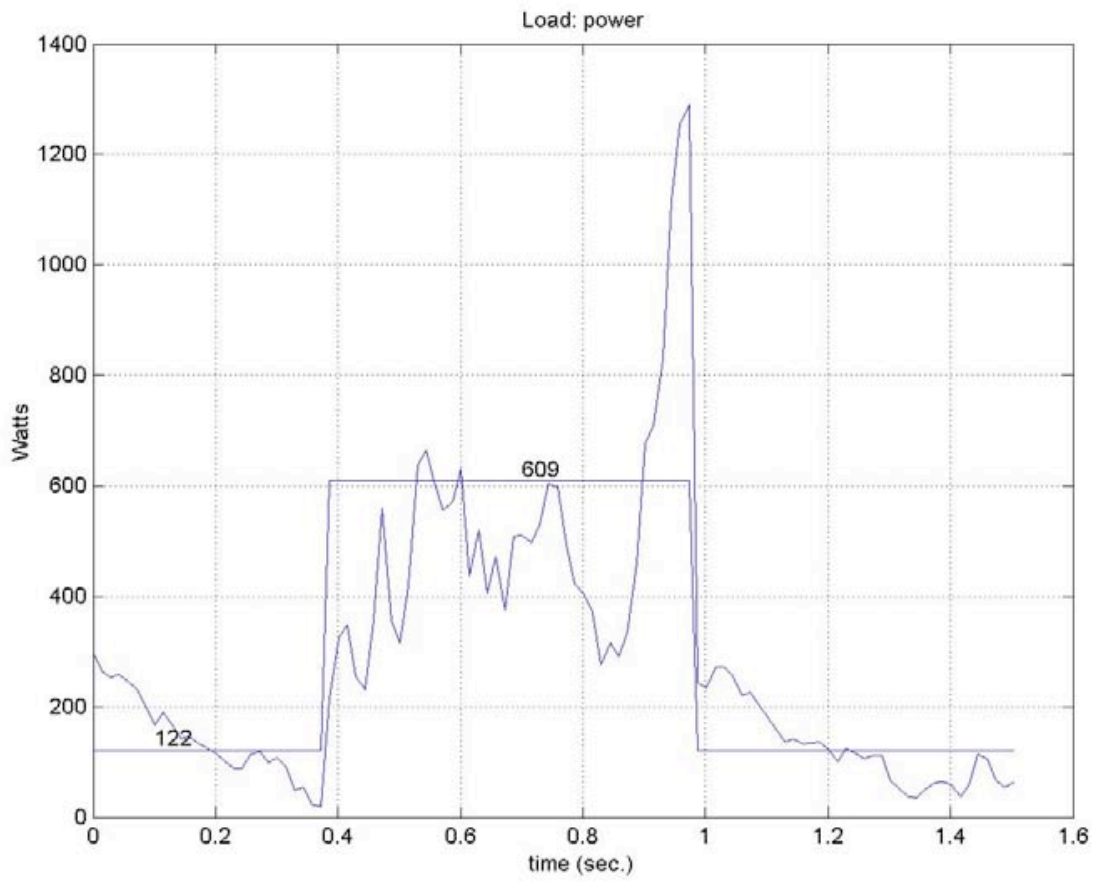

Fig. 80. Power requirement of pump. 


\section{ACKNOWLEDGEMENTS}

This report is the compilation of the efforts of multiple organizations that participated in this team effort. ORNL thanks the teams and team members that contributed to these efforts and the compilation of this report.

Support for this research was provided by DARPA under DOE/ORNL - DOD/DARPA InterFederal Agency Agreement No. 1868-HH27-X1. ORNL wishes to thank and acknowledge the direction and support of EHPA program managers Dr. Ephrahim Garcia and Dr. John Main and their staffs. 
APPENDIX A

\section{FEASIBILITY STUDY - CONTROLLER DESIGN}


This appendix is an excerpt from an ORNL exoskeleton feasibility study conducted for DARPA in 2000 (Jansen et al., 2000). This appendix provides background and the rational for the controls approach taken and is included here for completeness and the convenience of the reader.

\section{CONTROLLER ISSUES AND APPROACH}

While biped control for autonomous robots is still an active research area, its application to an exoskeleton is almost negligible because the balancing capability of the human operator can be transferred to the exoskeleton. The transfer of balancing capability from the operator to the exoskeleton is based on bilateral force reflection. Historically, the major application of bilateral force reflection controls has been for teleoperator (master-slave) systems. There are three general types of control strategies used in a teleoperator system: (1) position-position, (2) position-force, and (3) force-force control. The first control strategy, which was used on the Hardiman, position-position control, refers to the type of sensory information (in this case positional information) being used in the "outer" feedback loop of both the master and slave. Position-position control is commonly implemented in most teleoperator systems. Two teleoperators ORNL built in the 1980s, the M2 and the Advanced Servo Manipulators, are examples of this type of control strategy that is common for systems that have kinematically similar master-slave manipulators. For kinematically dissimilar master-slave systems, positionforce control is commonly used. For human amplifier (or extender) type systems, where the master and slave are one integral unit such that the human operator and the manipulator are attached to a common point and bilateral force reflection is the primary performance objective, force-force control is commonly used.

The purpose of this section is to define the fundamental control objectives and outline the basic control topology associated with the design of any mechanical exoskeleton system. Issues, such as the amount of mass felt by the human operator, minimum feedback gains, determining the critical sensory feedback signals, and reflection of disturbance forces back to the operator will be discussed.

To avoid undue complexity, a simple 1-DOF system, shown in Fig. 81, will be examined to better understand the salient issues. Figure 81 shows two types of actuators: the first is the human actuator in parallel with the mechanical actuator (such as a hydraulic cylinder). Force sensing is represented by the oval symbols, and force sensing at the human and the mechanical actuator are shown. It should be mentioned that force sensing at the foot instead of at the mechanical actuator is also possible and would be preferred, but for the sake of simplicity, the sensing also shown in Fig. 81, will be useful for discussion. The mass of the human is represented by $M_{h}$, the exoskeleton mass is represented by $M_{e x}$, and the payload mass is represented by $M_{p}$.

Three states will be considered in the analysis. The first is when the (mechanical) foot is on the ground with the exoskeleton system at rest. The second is when the (mechanical) foot first impacts the ground and the last when the 1-DOF system is off the ground. Linear analysis (in particular Laplace Transform theory with the s operator) will be assumed to adequately describe the basic physics for a fundamental understanding of a mechanical exoskeleton system. For state 1 , where the exoskeleton system is in contact with the ground and initially at rest (i.e., impact is 
ignored and the initial velocity is zero), the governing dynamic equations of the overall mechanical system can be described as

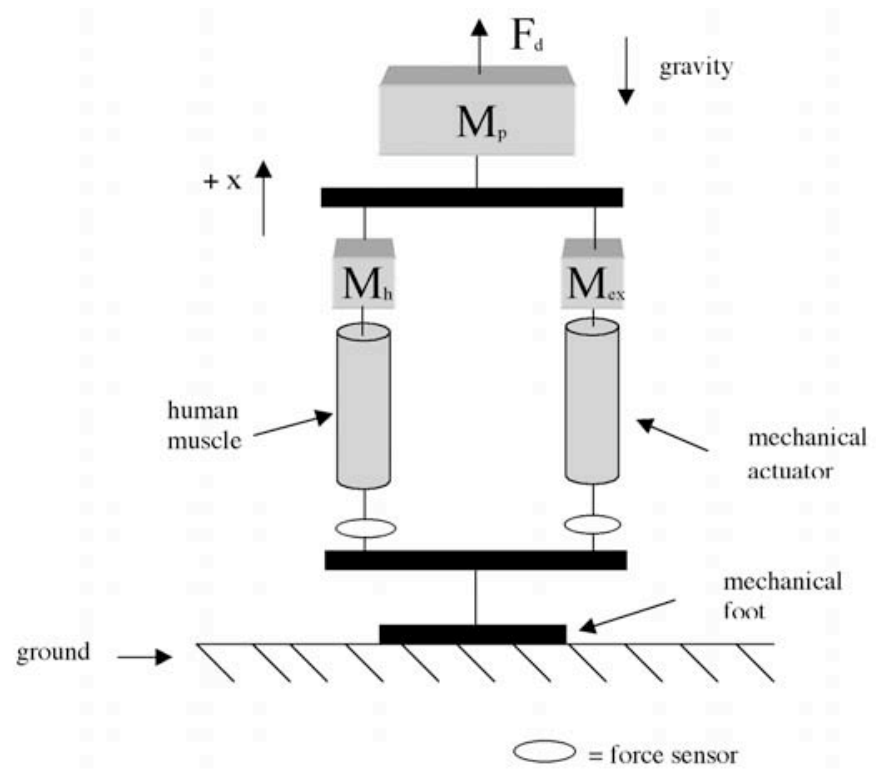

Fig. 81. One DOF exoskeleton system.

$$
M_{t} s \dot{x}=F_{h}+F_{e x} \square M_{t} g+F_{d}
$$

where $M_{t}$ is the total mass and equal to $M_{p}+M_{h}+M_{e x}$, g is the gravity constant, and $F_{d}$ is an external disturbance force. Assuming that the mechanical actuator can be set as a force-based actuator (e.g., current control in an electric motor directly controls the shaft torque), that is, $\mathrm{F}_{\mathrm{ex}}=\mathrm{K}_{\mathrm{ex}} \mathrm{i}_{\mathrm{ex}}$ where $\mathrm{K}_{\mathrm{ex}}$ is the force constant and $\mathrm{i}_{\mathrm{ex}}$ is the input drive signal.

By defining the desired human admittance, $\mathrm{Y}_{\mathrm{h}}{ }^{\text {des }}$, as

$$
\mathrm{Y}_{\mathrm{h}}^{\mathrm{des}}=\left.\frac{\dot{\mathrm{x}}}{\mathrm{F}_{\mathrm{h}}}\right|_{\mathrm{F}_{\mathrm{d} \mathrm{D}}=0}
$$

where $F_{d^{\prime}}=F_{d}-M_{t} g$, the desired human admittance can be thought of as the amount of force a human operator has to apply to achieve a given motion velocity. One would like this admittance to be close to a user specified mass and viscous force value that is limited only by power and bandwidth bounds. If one could achieve an arbitrary human admittance such as

$$
\mathrm{Y}_{\mathrm{h}}^{\text {des }}=\frac{1}{\mathrm{M}_{\mathrm{eff}} \mathrm{S}+\mathrm{K}_{\text {veff }}}
$$

where $M_{\text {eff }}$ is the effective mass and $K_{\text {veff }}$ is associated with the effective viscous force, then a detailed understanding of the human neuromuscular control system is unimportant since humans already know how to respond to these environmental forces. To achieve force amplification, an outer control loop will generate an error signal, E, as shown in Fig. 82, where $\mathrm{K}_{\mathrm{h}}$ is the force 
amplification factor. The goal of the controller is to drive the error term to zero creating a relationship between the human force and the exoskeleton force by the force amplification factor.

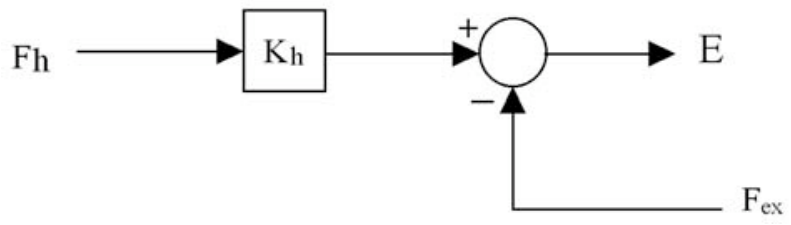

Fig. 82. Outer force control loop.

The remaining issue is the mapping of the error signal, $\mathrm{E}$, to the drive signal, $\mathrm{i}_{\mathrm{ex}}$. One possible control topology is shown in Fig. 83, where the controller can be represented as

$$
\mathrm{i}_{\mathrm{ex}}=\mathrm{K}_{\mathrm{c} 2} \underset{\mathrm{c}}{\square} \mathrm{G}_{\mathrm{c}}\left(\mathrm{K}_{\mathrm{h}} \mathrm{F}_{\mathrm{h}} \square \mathrm{F}_{\mathrm{ex}}\right) \square \mathrm{K}_{\mathrm{p}} \frac{\dot{\mathrm{x}}}{\mathrm{s}}=
$$

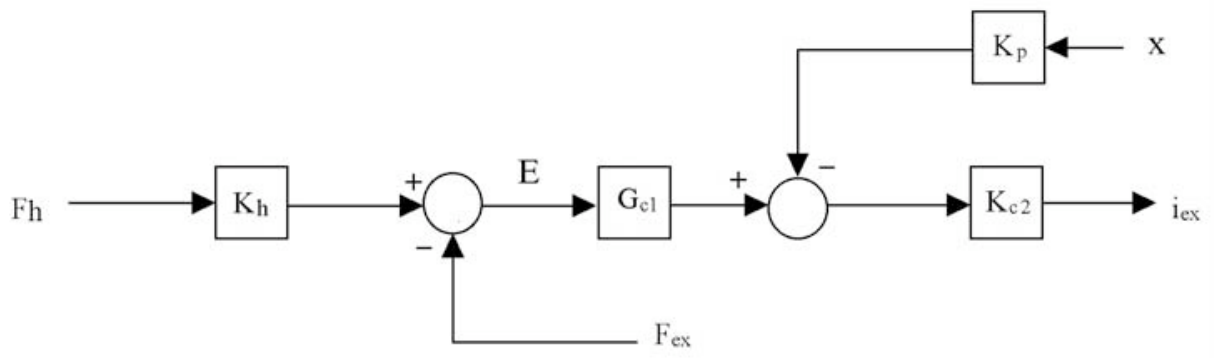

Fig. 83. Exoskeleton controller.

From the combined physical system and controller, the actual admittance of the exoskeleton, $\mathrm{Y}_{\mathrm{h}}{ }^{\mathrm{ex}}$, as felt by the human operator, can be shown to be

$$
\mathrm{Y}_{\mathrm{h}}^{\mathrm{ex}}=\left.\frac{\dot{\mathrm{x}}}{\mathrm{F}_{\mathrm{h}}}\right|_{\mathrm{F}_{\mathrm{d}}=0}=\frac{\mathrm{s} / \mathrm{\theta}+\left(1+\mathrm{K}_{\mathrm{h}}\right) \mathrm{G}_{\mathrm{cl}} \mathrm{K}_{\mathrm{c} 2} \mathrm{~K}_{\mathrm{ex}} \mathrm{\theta}}{\mathrm{M}_{\mathrm{t}} \mathrm{s}^{2}\left(1+\mathrm{G}_{\mathrm{c} 1} \mathrm{~K}_{\mathrm{c} 2} \mathrm{~K}_{\mathrm{ex}}\right)+\mathrm{K}_{\mathrm{p}} \mathrm{K}_{\mathrm{c} 2} \mathrm{~K}_{\mathrm{ex}}}
$$

If $\mathrm{G}_{\mathrm{c} 1}=1 / \mathrm{s}$ and the $\mathrm{K}_{\mathrm{c} 2}$ gain is made large enough (this is where a careful overall design is critical), then it can be shown that over the user-specified frequency range, the actual admittance felt by the human operator is

$$
\mathrm{Y}_{\mathrm{h}}^{\mathrm{ex}}(\mathrm{j} \square) \square \square \frac{1+\mathrm{K}_{\mathrm{h}}}{\mathrm{M}_{\mathrm{t}} \mathrm{s}+\mathrm{K}_{\mathrm{p}}}
$$

This shows that the effective mass is $M_{\text {eff }}=M_{\mathrm{l}} /\left(1+K_{h}\right)$ and the effective viscous coefficient is $\mathrm{K}_{\text {veff }}=\mathrm{K}_{\mathrm{p}} /\left(1+\mathrm{K}_{\mathrm{h}}\right)$. By controlling the value of force amplification gain $\mathrm{K}_{\mathrm{h}}$ and the feedback gain $\mathrm{K}_{\mathrm{p}}$, arbitrary amounts of effective inertia and viscous friction forces can be felt by the human operator. As an example, say that we want the human operator to feel only his or her mass and none of the payload or the exoskeleton system mass, then the amplification gain would have to be $K_{h}=\left(M_{e x}+M_{p}\right) / M_{h}$. By increasing the force amplification even further, a portion of the human operator mass can also be reduced by energy provided to the exoskeleton actuator. 
Likewise, it can be shown that the gravitational and disturbance forces that are reflected back to the human operator, called the overall disturbance force (i.e., $F_{d}$ ), and assuming that the exoskeleton system is not moving, is related to the human force by

$$
\left.\frac{\mathrm{F}_{\mathrm{d}}}{\mathrm{F}_{\mathrm{h}}}\right|_{\dot{\mathrm{X}}=0} \square \frac{1}{1+\mathrm{K}_{\mathrm{h}}}
$$

over the user-specified frequency range. This indicates that force reflection is simply a function of the amplification gain and is similar to the ratios of the effective mass and viscous forces.

For state 2, where the exoskeleton system impacts the ground, the only difference between state 2 and state 1 is that the initial velocity is assumed to be nonzero. Equation (7) can be modified to show this change

$$
\mathrm{M}_{\mathrm{t}} \mathrm{s} \dot{\mathrm{x}}=\mathrm{F}_{\mathrm{h}}+\mathrm{F}_{\mathrm{ex}} \square \mathrm{M}_{\mathrm{t}} \mathrm{g}+\mathrm{F}_{\mathrm{d}} \mathrm{M}_{\mathrm{t}} \dot{\mathrm{x}}(0)
$$

(Note that this equation is in the s-domain and not the time domain). In addition, the disturbance force is modified to

$$
F_{d \square}=\square M_{t} g+F_{d}+M_{t} \dot{x}(0)
$$

All the results for state 1 can now be applied to state 2, while the only change, the additional momentum force, is because of a nonzero impact velocity. This impact force will be felt by the human operator, but at a reduced level, and can be further reduced by means of suitable mechanical compliance in the exoskeleton structure.

If one examines how the disturbance force can affect the exoskeleton velocity, it can be shown through reasoning similar to the discussion for state 1 , that

$$
\left.\frac{\dot{\mathrm{x}}}{\mathrm{F}_{\mathrm{d}}}\right|_{\mathrm{F}_{\mathrm{h}}=0} \square \frac{1}{\mathrm{M}_{\mathrm{t}} \mathrm{s}+\mathrm{K}_{\mathrm{p}}}
$$

over the user-specified frequency range, where the benefits of the computer-controlled viscous damping term $\mathrm{K}_{\mathrm{p}}$ becomes clear; it dissipates the impact force. Impact forces can be damped to specific target values by the controller; in addition, the mechanical structure can be designed to provide further system damping.

For state 3, where the exoskeleton is off the ground, all ground reactions are obviously zero which forces the governing dynamic equations represented originally by Eq. (7) to change to the following:

$$
M_{t} s \dot{x}=\square M_{t} g+F_{d}+M_{t} \dot{x}(0) \text { and } F_{h}+F_{e x}=0
$$

(Note that these equations are in the s-domain and not the time domain in regard to the initial velocity of the system). The actuator for this 1-DOF exoskeleton system cannot affect the trajectory of the system, and the human and exoskeleton forces add up to zero. The exoskeleton 
controller will try to force tracking between the human force and the exoskeleton force, which implies that the effective human force will be about zero (depending on the tracking performance of the controller). When the exoskeleton system is off the ground, the human feels almost no force and cannot affect the trajectory of the system. This is true only for this simple case, where only a 1-DOF example is being considered. For a two-legged system, the leg on the ground would provide the reaction force for the leg that is off the ground.

In summary, a 1-DOF system has been examined to study the feasibility of using a force-based control system to implement performance enhancement. An overview of controller issues, including force sensing, creating a relationship between the human and exoskeleton forces, the use of the human capability to respond to environmental forces, and the damping of impact forces have been addressed. It has been shown that by means of force-based feedback, the effective mass and disturbance and viscous forces as felt by the human operator of an exoskeleton, can be readily controlled. Performance is intrinsically limited only by the mechanical bandwidth and power limitations of the actuators, and the mechanical structure. While examined with a 1-DOF case, these concepts should readily extend to the multiple-DOF case.

\section{SAFETY AND FAULT DETECTION CONCEPTS}

Safety will be a prime concern in the eventual deployment of any exoskeleton. A common failure scenario in a hydraulic servomechanism is the failure of either the servovalve, drive electronics, or sensors (say a positional sensor like a resolver) causing a significant flow of fluid to the hydraulic actuator. Detection of this type of fault has to occur quickly because of the highbandwidth of an exoskeleton, which could result in significant motion within $150 \mathrm{~ms}$. For example, the required dynamic response could result in $80^{\circ}$ movement of the knee in $150 \mathrm{~ms}$ or less. The best-case human-reflex response time is 110 to $150 \mathrm{~ms}$. Thus, there is a significant potential for injury without adequate fault detection and response capability. Because of the human reaction times, it is unreasonable to expect that an operator could activate an emergency stop button in the time required to prevent injury in the worst-case scenario.

The general approach to error detection is through model-based anticipatory and trendforecasting schemes (such as Kalman predictive filtering), which can detect a system error during system transients (Tylee, 1983). Unfortunately, conventional methods cannot distinguish between a fault and system disturbance because of the short decision times available when a failure occurs and the unpredictable nature of disturbances acting on an exoskeleton (such as thermal drift in the drive electronics, mechanical vibrations, impact forces). This explains why such systems are not readily commercially available.

ORNL's approach has been to break apart the disturbance forces acting on the system (such as thermal drift in the drive electronics, mechanical vibrations, impact forces) from the overall nonlinear system response caused by a fault. Preliminary work indicates that this approach of partitioning of these forces on a real system can achieve response times around $200 \mathrm{~ms}$ (see Fig. 84). ORNL has demonstrated this approach and $200 \mathrm{~ms}$ response times on the Next Generation Munitions Handler (NGMH) system. The response times of the command valves are currently limited around $100 \mathrm{~ms}$ by the inductance-resistance time-constants. The eventual goal 
of the safety-fault detection system is to reduce the response times to as close to a $50 \mathrm{~ms}$ goal as possible.

Analysis of the current system indicates that for a hydraulic system, using special shut-off valves, which have reduced inductance-resistance time-constants, can significantly reduce the response time. Other factors, such as faster processing capabilities have a much less significant impact. Further research is needed regarding issues relating to system safety and approaches for further reducing response times. Current models and simulations, verified by data from an operating system (NGMH), provide a starting point for more detailed study of approaches to ensuring system safety.
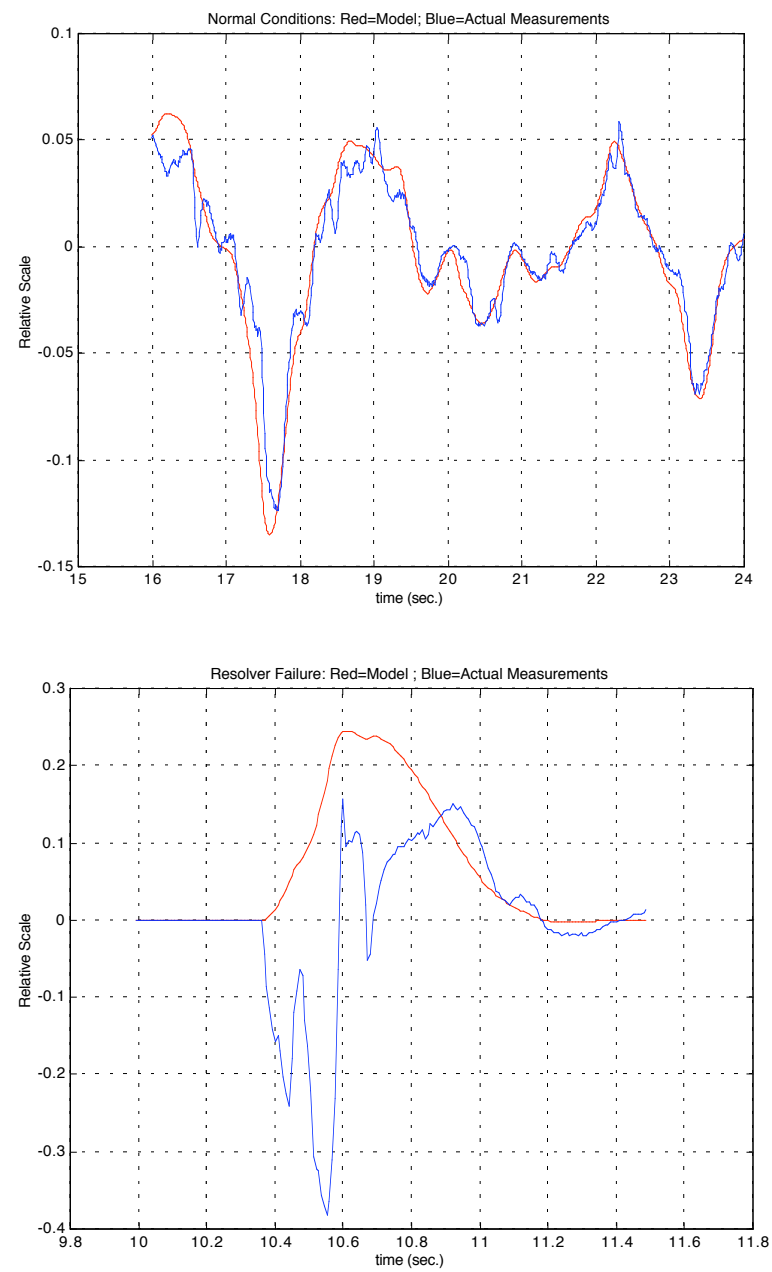

Fig. 84. NGMH fault detection (normal operation and sensor failure). 
APPENDIX B

\section{FEASIBILITY STUDY - DESIGN ISSUES}


This appendix is an excerpt from an ORNL exoskeleton feasibility study conducted for DARPA in 2000 (Jansen et al., 2000). This appendix provides background and rational for the design approach taken and is included here for completeness and the convenience of the reader.

\section{TECHNICAL APPROACH}

The design of the exoskeleton structure must address the fact that the structure's primary function is to support the payload and provide the mechanical interface with the operator, while at the same time, the function must be transparent to the operator and enable overall system energy efficiency. In essence, the exoskeleton is wrapped around the operator and supports the payload as well as its own load with the objective of minimizing disturbances exerted on the operator. Two basic design approaches will be discussed. The first is based on designing the exoskeleton to track the human operator joint by joint. The second approach is based on tracking the limb end-point (foot or hand), but not necessarily matching each joint. Regardless of the approach taken, the design of the exoskeleton should be highly anthropomorphic. While the overall form of the exoskeleton may not differ dramatically based on the approach taken, system complexity, including the number and location of joints, the number and location of attachment points, and the control system complexity, will all be affected.

In comparing these two approaches, consideration of some of the intricacies of human anatomy must be taken into account. First, the human arm and leg have redundant joints; thus, there are multiple joint configurations for each unique hand and foot position and/or orientation. To replicate this capability adds degrees of freedom and increasing system complexity. A study by the ARL detailing requirements for an exoskeleton suggests a minimum number of DOF (Table 6). Note that the recommendation includes 6 DOF for the leg (it does not include ankle abduction-adduction) and 7 DOF for the arm. The addition of a redundant degree of freedom will obviously increase the overall complexity of the control system.

Table 6. Minimum DOF for exoskeleton joints (Crowell, 1995)

\begin{tabular}{|l|l|l|}
\hline Joint & DOF & Description \\
\hline Foot & 1 & Extension for metacarpophalangela joint \\
\hline Ankle & 1 & Flexion-extension \\
\hline Knee & 1 & Flexion \\
\hline Hip & 3 & Flexion-extension, abduction-adduction, medial-lateral rotation \\
\hline Pelvis & 3 & Coronal, sagittal, and transverse plane rotations \\
\hline Spine segments & 3 & Flexion-extension lateral flexion, rotation \\
\hline Shoulder & 3 & Flexion-extension, abduction, medial-lateral rotation \\
\hline Elbow & 2 & Flexion, forearm pronation-supination \\
\hline Wrist & 2 & Flexion-extension, abduction-adduction \\
\hline
\end{tabular}

Co-locating joints such that both the human joint and the exoskeleton joint rotate about a common axis is difficult, especially at joints with multiple degrees of freedom, such as the hip, shoulder, wrist, and ankle. With a non-co-located joint, the likelihood of the exoskeleton impeding the motion of the operator is increased. This increases the difficulty of the design, especially when combined with the need to accommodate a broad range of operator sizes. The 
addition of a redundant joint, while adding complexity, especially to the control system, can aid in avoiding configurations where the exoskeleton does in fact impede the operator.

To follow or track, the human operator on a joint-by-joint basis requires attachment and sensing at or near the joint. For example, to track the knee, attachment above and below the knee would be required. As joint-by-joint tracking implies a position-position control loop, position sensing of both the human and exoskeleton joints would also be required. Complicating this is the fact that the centers of rotation for human joints, such as the knee, are not fixed. Replicating these types of joints mechanically is difficult, and the likely result is tracking errors, which will have some impact on the interface at the attachment points.

The second approach, tracking the limb end-point, has advantages over the joint-by-joint tracking approach. First, attachments are not required at each joint. In particular, no attachment is required for the elbow and knee. The number of degrees of freedom can be limited to 6 for each limb, thus reducing complexity and weight. Accurately tracking of each individual joint is not an issue, eliminating the need to accurately sense the position of each human joint and to accommodate the motion of complex joints such as the knee. Significant reduction in mechanical complexity is achieved in this approach at the expense of increased complexity of the control system. The remaining discussion is based on the results of these comparisons, in an effort to address other design issues affecting feasibility.

\section{STRUCTURE}

Design of a transparent exoskeleton is affected by many parameters such as response bandwidth, sensing points, soft-tissue interfaces, and amplification schemes, in addition to key structural parameters, including kinematics, load-transfer points, and the determination of actuated versus non-actuated joints. While the design of the exoskeleton should be highly anthropomorphic, a 1:1 correspondence between each of the exoskeleton and operator joints is not necessary. It is essential, however, that these joints be located in as close of proximity as possible, although not necessarily co-located.

Co-locating joints, such as the hip and wrist, were evaluated to determine the design trade-offs related to the structure. The most significant advantage of co-locating the joints is reduced tracking errors. The trade-off is in the increased complexity, as can be seen in the co-located hip joint as seen in Fig. 85. A special configuration that allows for remote centers of rotation (which are co-located with the body's center of rotation), limits the range of motion achievable without interfering with the body, and greatly increases the difficulty in the design of bearings. The fact that the hip requires the simultaneous co-location of three DOF, adds tremendously to the mechanical complexity in achieving the desired range of motions. Figure 85 shows the medial and lateral rotations, as well as the hip abduction. It can be seen in the hip abduction (third frame) that the motion causes an interference with the body. After investigating a number of options, it became apparent that co-locating a joint as elaborate as the human hip was extremely difficult, with complexity that rendered such a design impractical. A non-co-located hip design, with linear actuators, is shown in Fig. 86. It should be noted that these figures represent concepts and that further detailed design would obviously be required. 

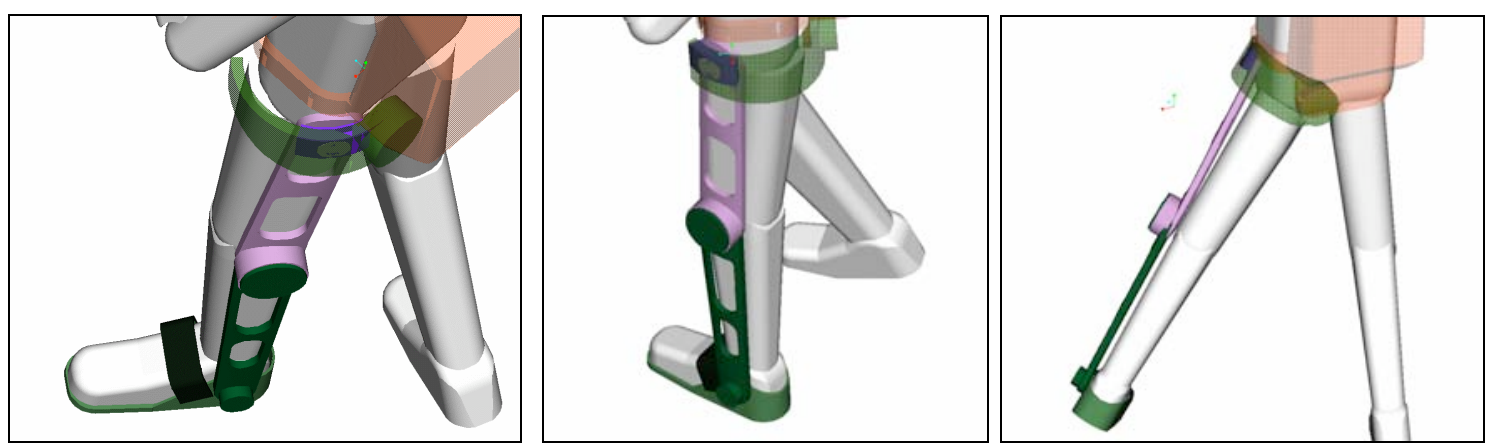

Fig. 85. Co-located hip.

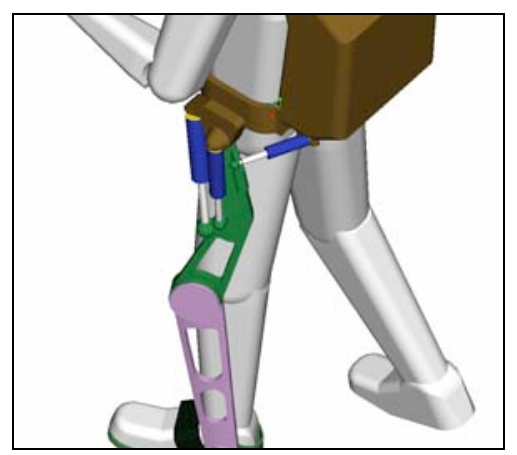

Fig. 86. Non-co-located hip with linear actuators.

Co-locating of joints is also an issue with the arm design as well. Due to complexity and range of motion limitations, the shoulder does not lend itself to co-location, similar to the hip. However, examination of the elbow joints forearm pronation-supination, which gives the wrist roll type of motion, indicates that co-locating of that joint can be accomplished while simplifying the design (Fig. 87).
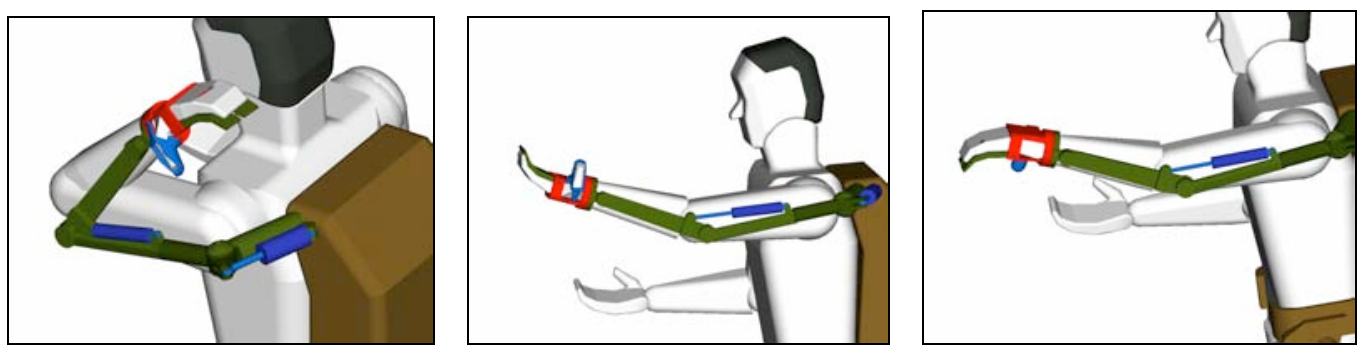

Fig. 87. Shoulder and arm structure.

Table 6 lists a minimum of 6 DOF per leg. A couple of points can be made concerning the number of DOF. It may be tempting to eliminate the hip medial-lateral rotation to simplify the design, as has been done in some proposed concepts. This, however, introduces a complication that would require further evaluation. Without this DOF and likewise, without the ankle abduction-adduction, the foot is constrained to remain in the same plane as the leg. This has implications with regards to changing direction, and at the very least will require slipping of the foot and may in fact alter the normal manner in which one changes direction. 
Joint design type was also investigated. The issue is to determine where to use rotary or linear actuators. Compactness of the design and range of motion are the determining criteria. The selection of either rotary or linear actuators is highly dependent on the joint. In general, rotary actuators appear to be more applicable to joints where more than $90^{\circ}$ range of motion is required. Linear actuators have an advantage with respect to the ease of measuring force. The knee flexion, for instance, could be done using either type of joint. The ankle flexion-extension, although it is less than $90^{\circ}$ may be more appropriately done with a rotary joint (Fig. 85). Ball and socket joints such as the hip in Fig. 86 would require linear joints.

As stated previously, minimization of power and energy requirements is crucial to the development of a truly "fieldable" exoskeleton system. An emphasis on optimizing the design of the structural elements to minimize system weight is an important aspect in the overall systems approach. The criteria for sizing the structural elements must take into consideration not only stresses, but structural stiffness as well. For the anticipated load range, stiffness is the determining factor in the sizing of the structural elements. Thus, minimizing the size of structural elements must be traded off with the requirement of maintaining structural stiffness.

While detailed modeling and analysis will be required to optimize this trade-off, preliminary analysis indicates that adjustable aluminum channel members can be used for the upper and lower links, respectively. Based on this analysis, assuming a $136 \mathrm{~kg}$ (300 lb) payload and a minimum structural natural frequency of $10 \mathrm{~Hz}$, an approximate weight for the structural system was calculated to be on the order of $12 \mathrm{~kg}$ (excluding actuators). The payload included the weight of the exoskeleton, assumed to be on the order of $32 \mathrm{~kg}$ (70 lb) and an effective payload of $105 \mathrm{~kg}(230 \mathrm{lb})$. The effective payload, which could take the form of either a backpack or an item manipulated using the exoskeleton arms, was used to size both the arms and the legs, which must transmit the load in either case. A study on the effects of increased payload showed that doubling of the payload had the affect of increasing the approximate weight of the structure by about $40 \%$ (excluding actuators).

For an exoskeleton to move from the laboratory to the field, the structure must be adjustable to accommodate a wide range of operator sizes. The goal should be able to accommodate from the $5 \%$ female to the $95 \%$ male. Adjustments will be required for both the upper and lower leg and arm links. Adjustment will also be required to accommodate for variances in torso proportions as well.

\section{ATTACHMENT}

Attachment of the exoskeleton is extremely difficult because of the close coupling required between the machine and the operator and the sensitivity of soft tissues, which are susceptible to bruising and damage with pressures as low as 0.1 atm over a few hours. There are considerable knowledge and experience embedded in the fields of orthotics and prosthetics, which have been addressing the problems of applying significant forces to the skeleton while keeping skin loading to acceptable levels.

As stated previously, the exoskeleton should be highly anthropomorphic - that joints should be located in as close of proximity as possible, although not necessarily co-located and that a 1:1 
correspondence between each of the exoskeleton and operator joints is not necessary. Therefore, the number of attachment points can be kept to a minimum by attaching at the limb extremities (feet and hands) and at the waist and shoulder. Thus, the leg or arm would be controlled based on the force input at the extremities as opposed to matching positions of each joint. By minimizing the number of attachment points, the total number of required DOF and actuators can be reduced, and the difficult task of mechanically replicating the motions of the human knee, which does not have a fixed center of rotation, can be avoided. The trade-off for simplifying the mechanical design is increased difficulty in the design of the control system (i.e., going from a joint-to-joint to a Cartesian control scheme).

It is important to provide a reliable and comfortable attachment at the human foot to the exoskeleton (Fig. 88). In this concept, soldiers outfitted for exoskeleton augmentation will wear boots that have built-in rapid-release attachment points. The boots will permit normal bending of the foot at the toes and will not create any additional human-tissue contact points. When not attached to the exoskeleton, the boots will feel and perform like ordinary boots. The coupling at the foot includes sensing of both the operator and the total system forces. These forces will be measured using multi-axis, force-torque sensors. The design will ensure that forces transmitted from the human foot to the exoskeleton can be accurately and unambiguously measured. The role of this force sensor was discussed in greater detail in the control section.

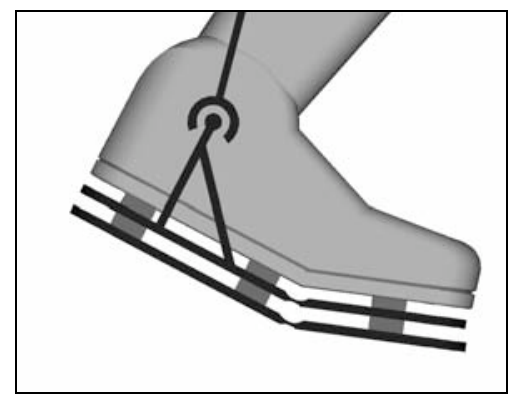

Fig. 88. Foot attachment schematic.

Attachment at the waist and shoulder areas would use a belt and shoulder straps, which are normal contact pressure areas. A major design issue would be to maintain comfort and acceptable loading over extended periods of time.

\section{CONCEPTUAL ENHANCEMENTS}

Some additional conceptual ideas that could enhance the design of an exoskeleton and thus potentially increase the likelihood of successfully developing an exoskeleton have been considered: first, the concept of using passive joints to reduce system complexity, and next, the concept of reconfiguration and multiple mode operation.

\section{Passive Joints}

While this study is focused on determining the feasibility of a powered exoskeleton, there may be opportunities to use passive joints as opposed to actuated joints in certain instances. Obviously, there is a significant advantage in weight and energy savings and reliability and cost 
in using passive joints where appropriate to simplify the system. While the actual determination of the joints that could be made passive would require more detailed analysis than the scope of this study allows, there are two joints that stand out as possible candidates. The metacarpophalangeal joint, as seen in Fig. 85, is the most likely candidate to be made passive. The hip-rotation joint is another possibility.

\section{Reconfiguration and Multiple Mode Operation}

The possibility of a modest reconfiguration of the system to allow a low power mode of operation was also investigated. In particular, a lower power, ballistic-type walking mode was investigated. Such mode would be advantageous for tasks such as long marches and could enhance operating times. Emulating a straight-leg ballistic mode is shown in Fig. 89. In this mode, the knees would essentially be locked and a thigh prismatic joint would be used to lengthen and shorten the legs, which would be moving with a pendulum type motion. Obviously, in such a mode close tracking of the knee is not a concern, but conservation of energy is the primary motivation. The trade-off for energy efficiency is the addition of the prismatic joint.

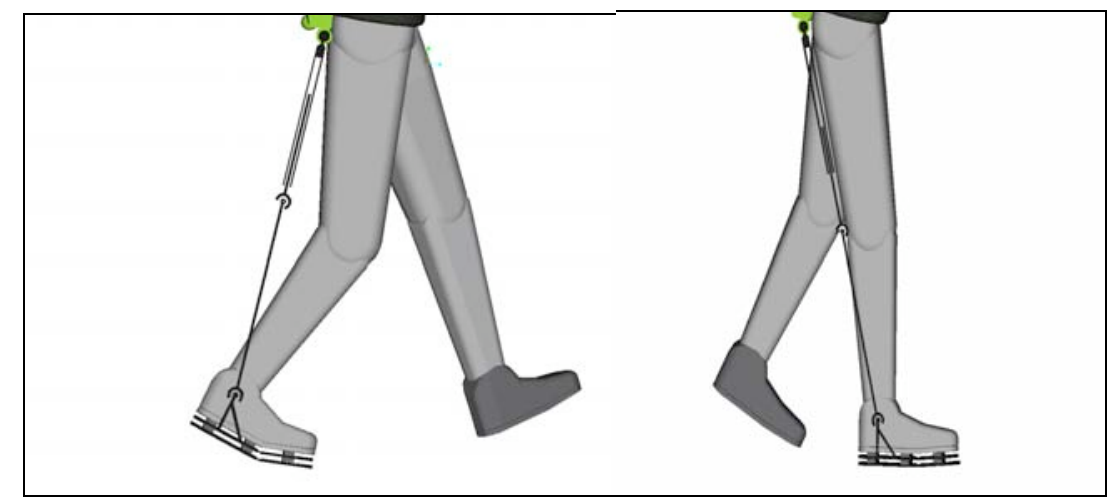

Fig. 89. Straight-leg ballistic mode.

The addition of a thigh prismatic joint (Fig. 90) could add other advantages. These include the minimization of tracking errors between the operator and exoskeleton legs, thereby reducing the risk of the exoskeleton impeding operator motion and allowing for a "tighter fitting" exoskeleton. The prismatic joint also compensates for pelvic-tilt-type motions, which were not easily accommodated with rotational joints.
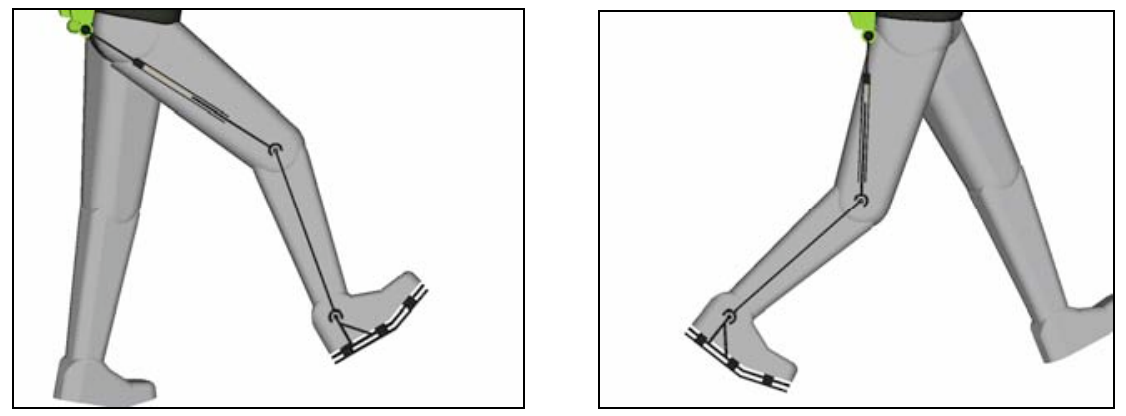

Fig. 90. Leg structure schematic demonstrating thigh prismatic joint. 


\section{APPENDIX C}

POWER AND TORQUE DATA 
This appendix contains the data from the biomechanics analysis performed to determine exoskeleton power and torque requirements that was conducted by ARL (Crowell, et al., 2002).
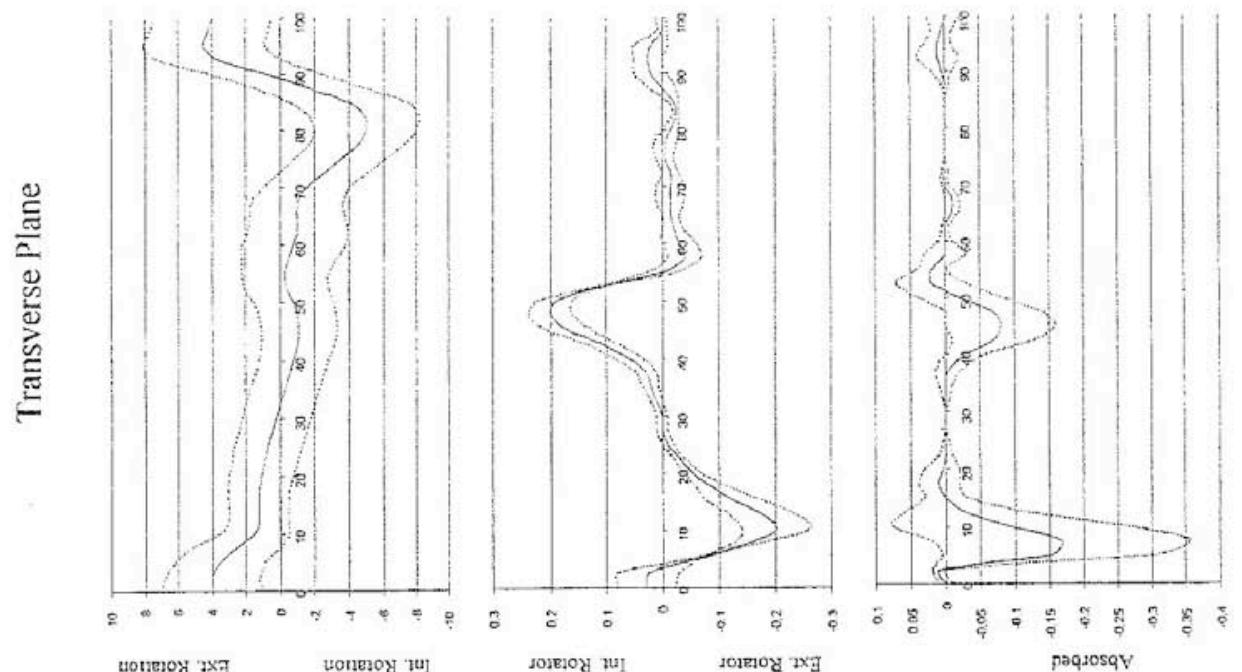

$\frac{0}{0}$
0
$=0$
0
$\overline{0}$
0
0
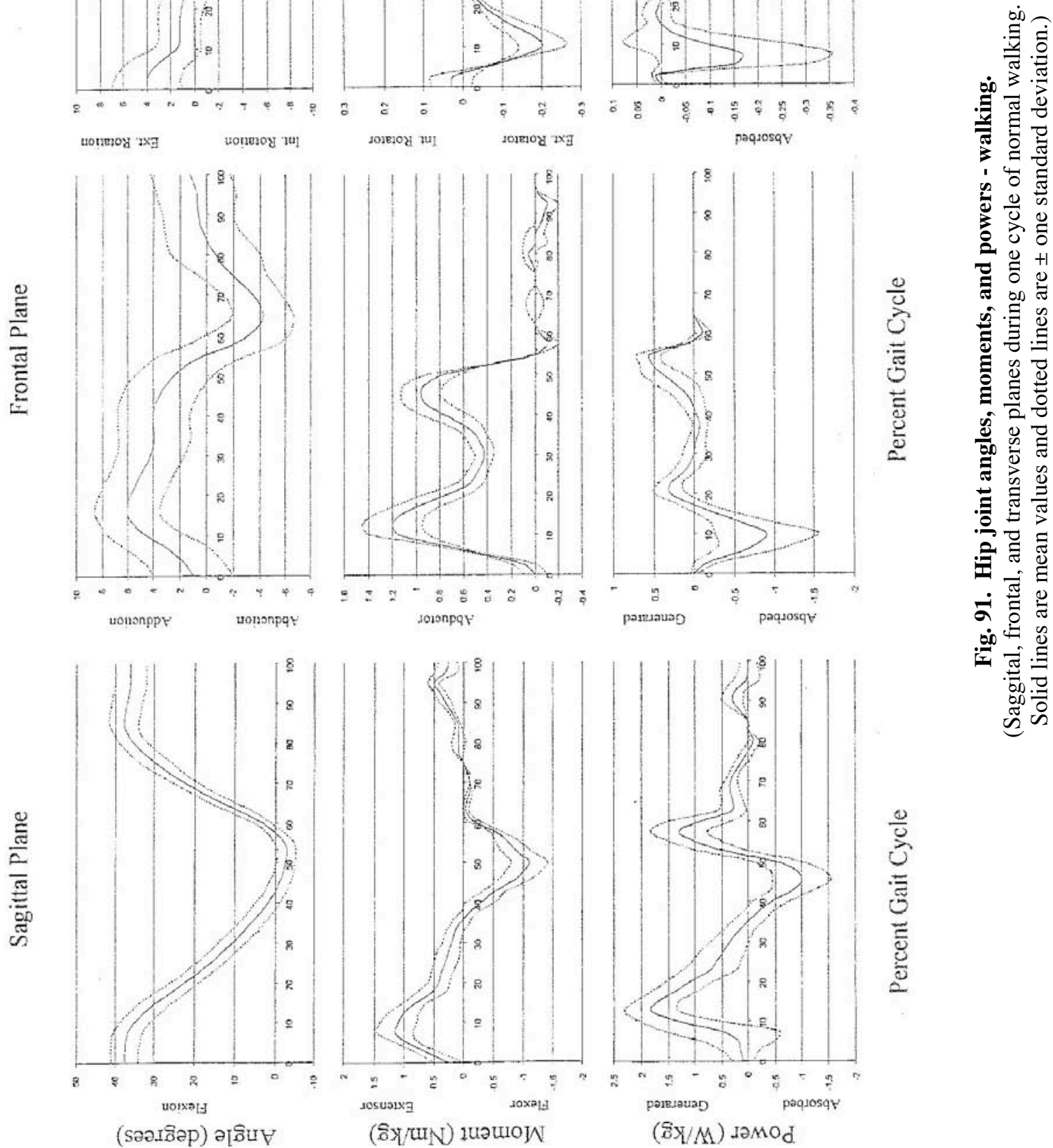

(รəวเอิอр) ว[ธินท

(气ิУ/UN) 2UวшоN

(อХ//M) IәMOC 


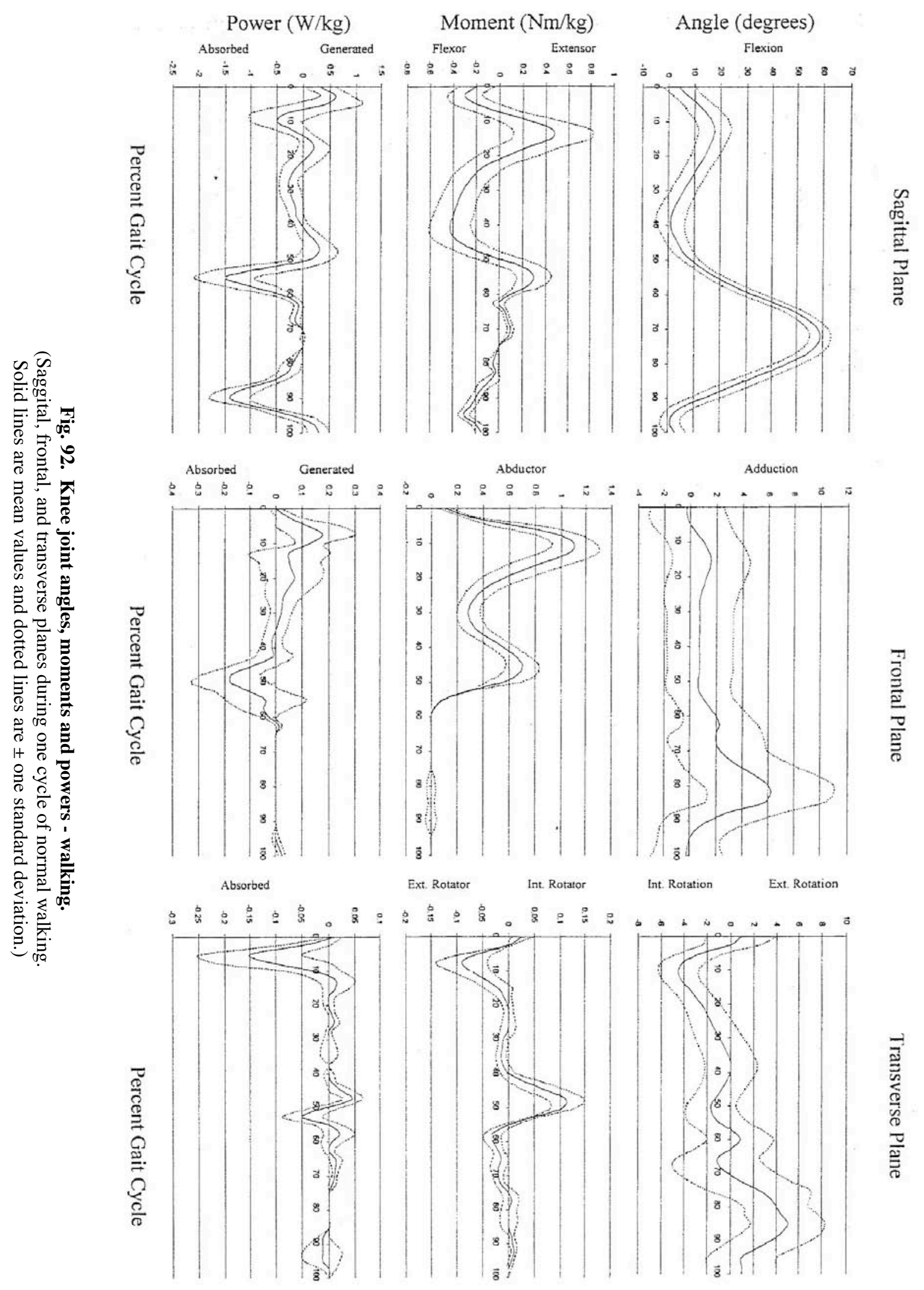



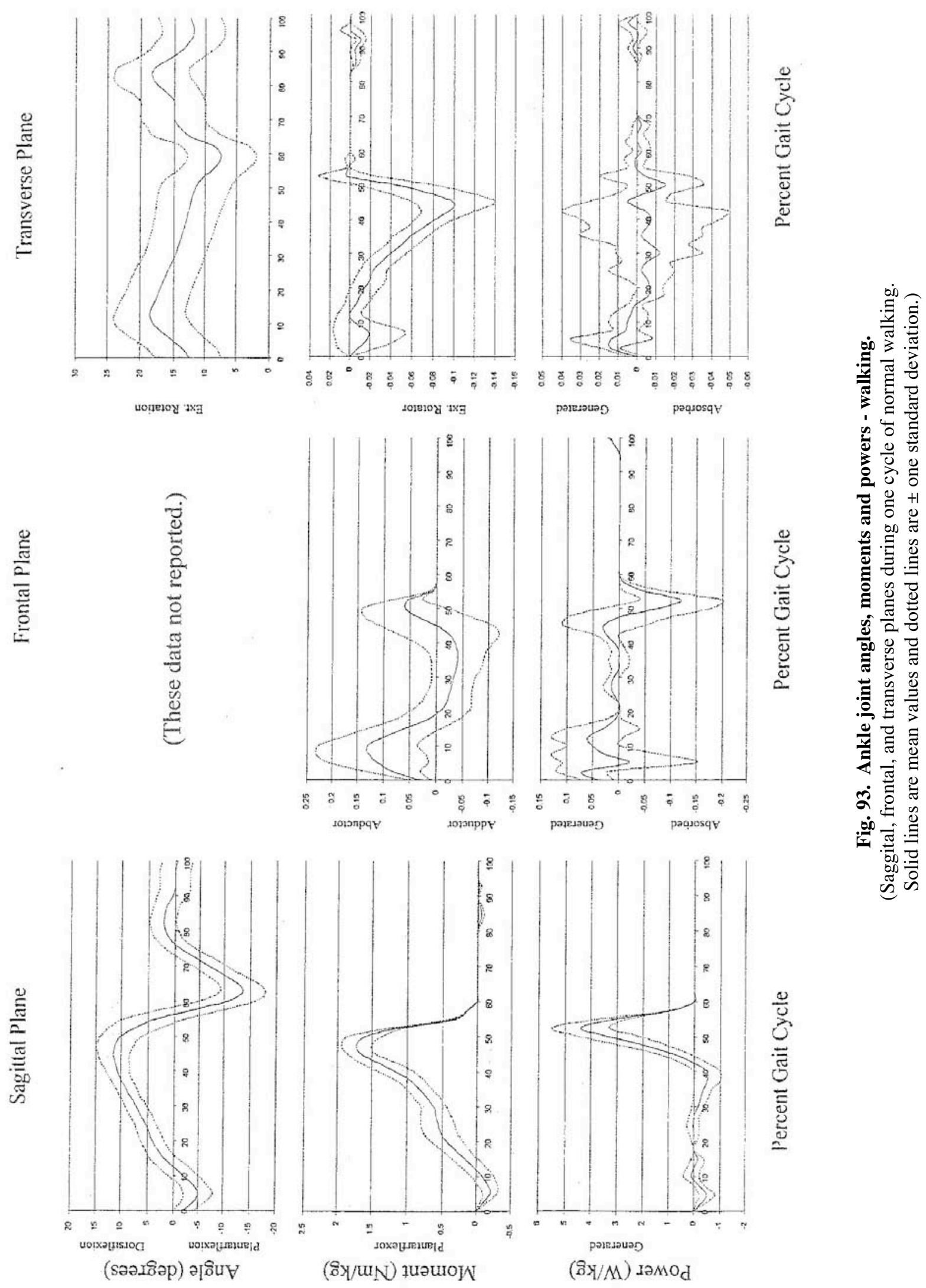


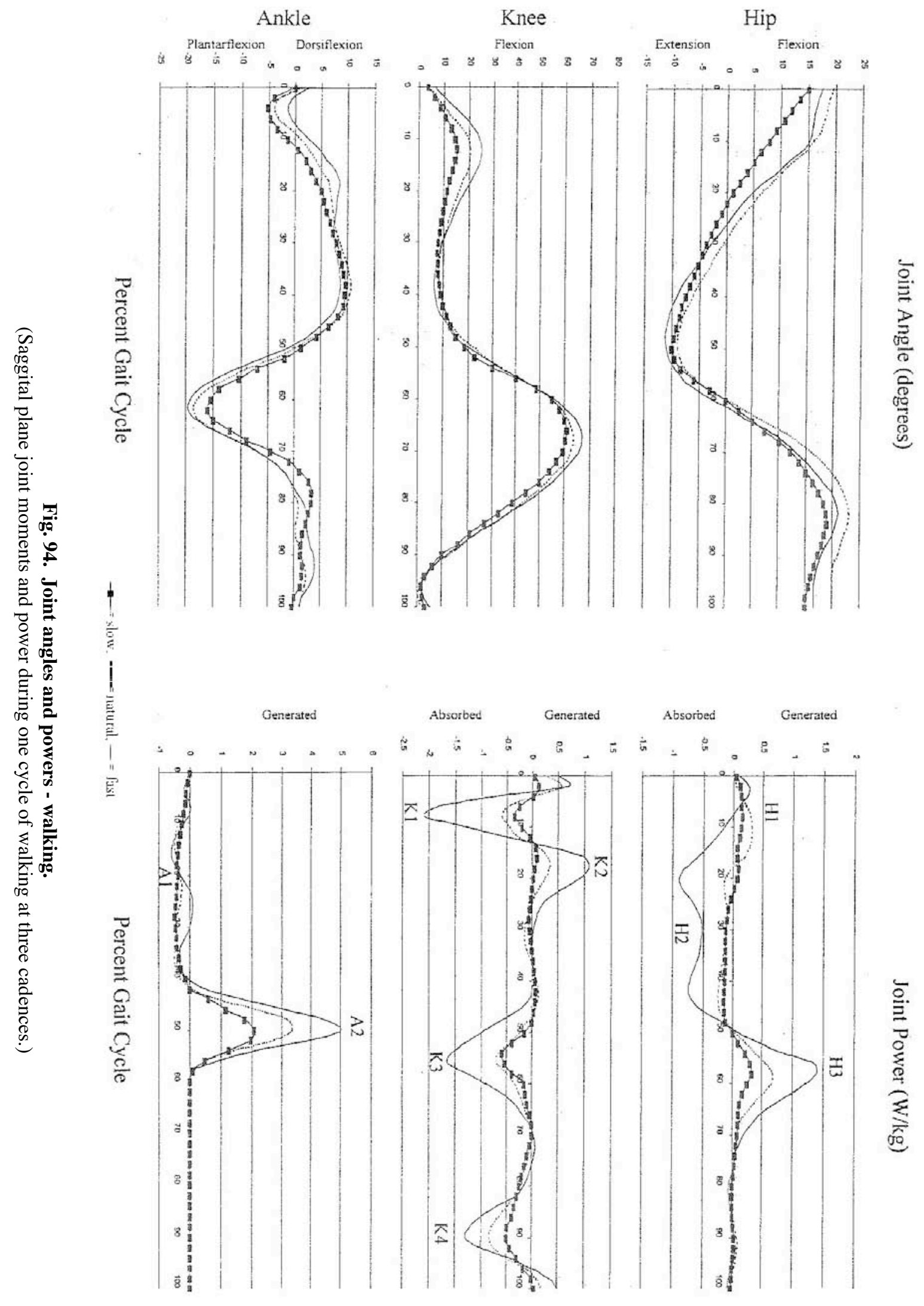



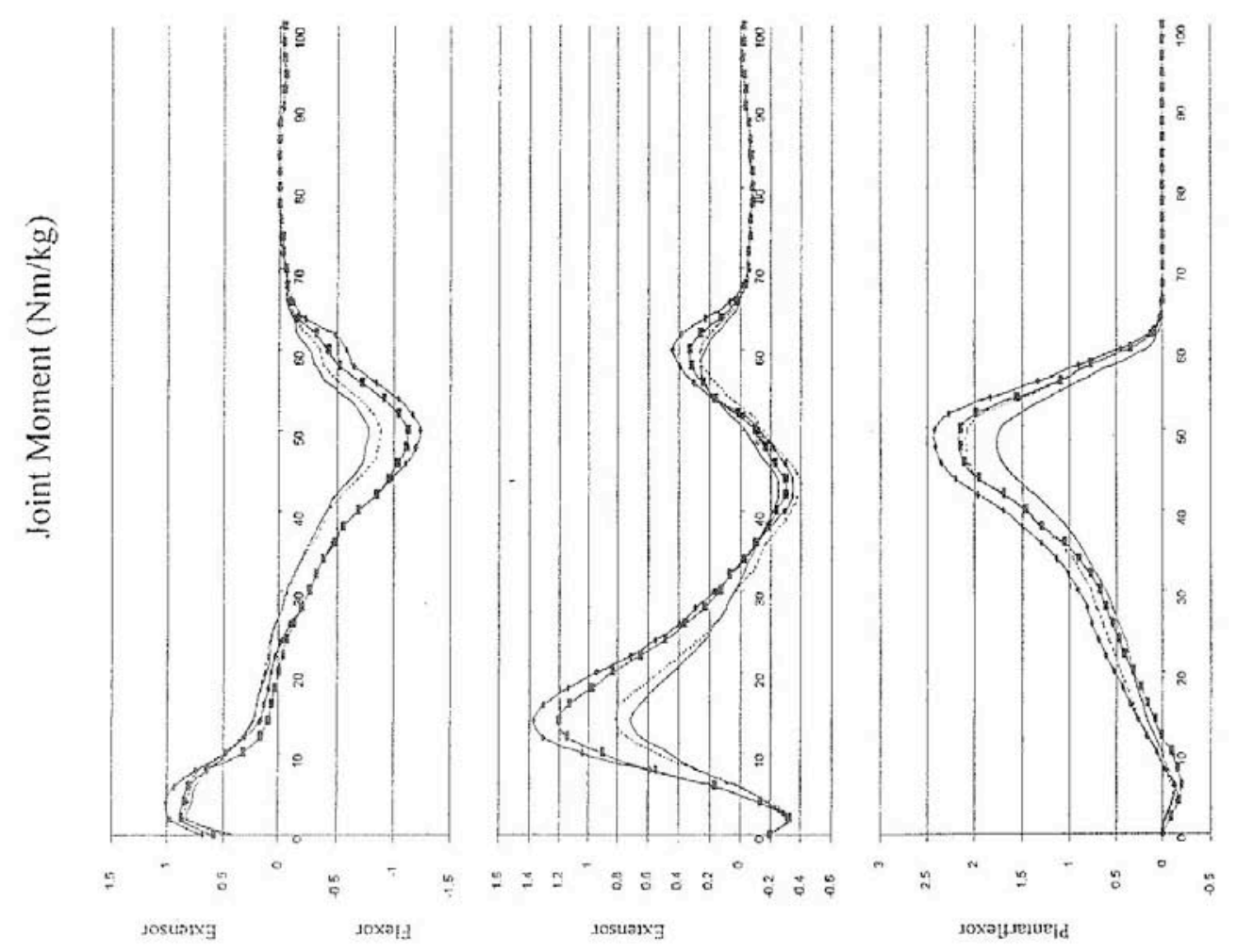

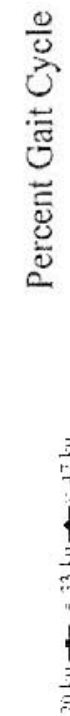

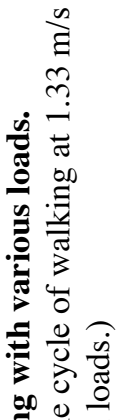

+

势

+1 每

韭 $\quad$ के

1

类要

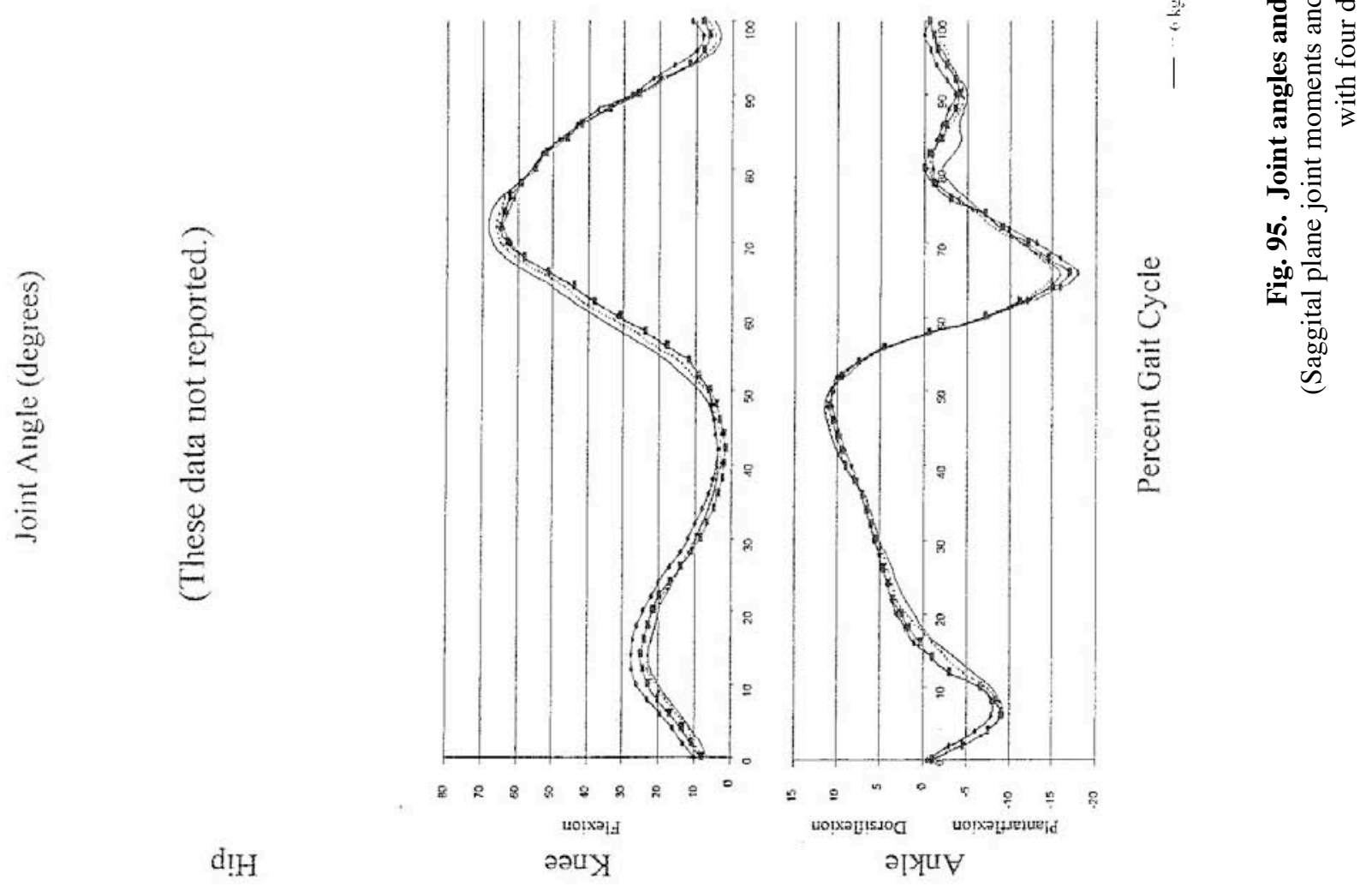




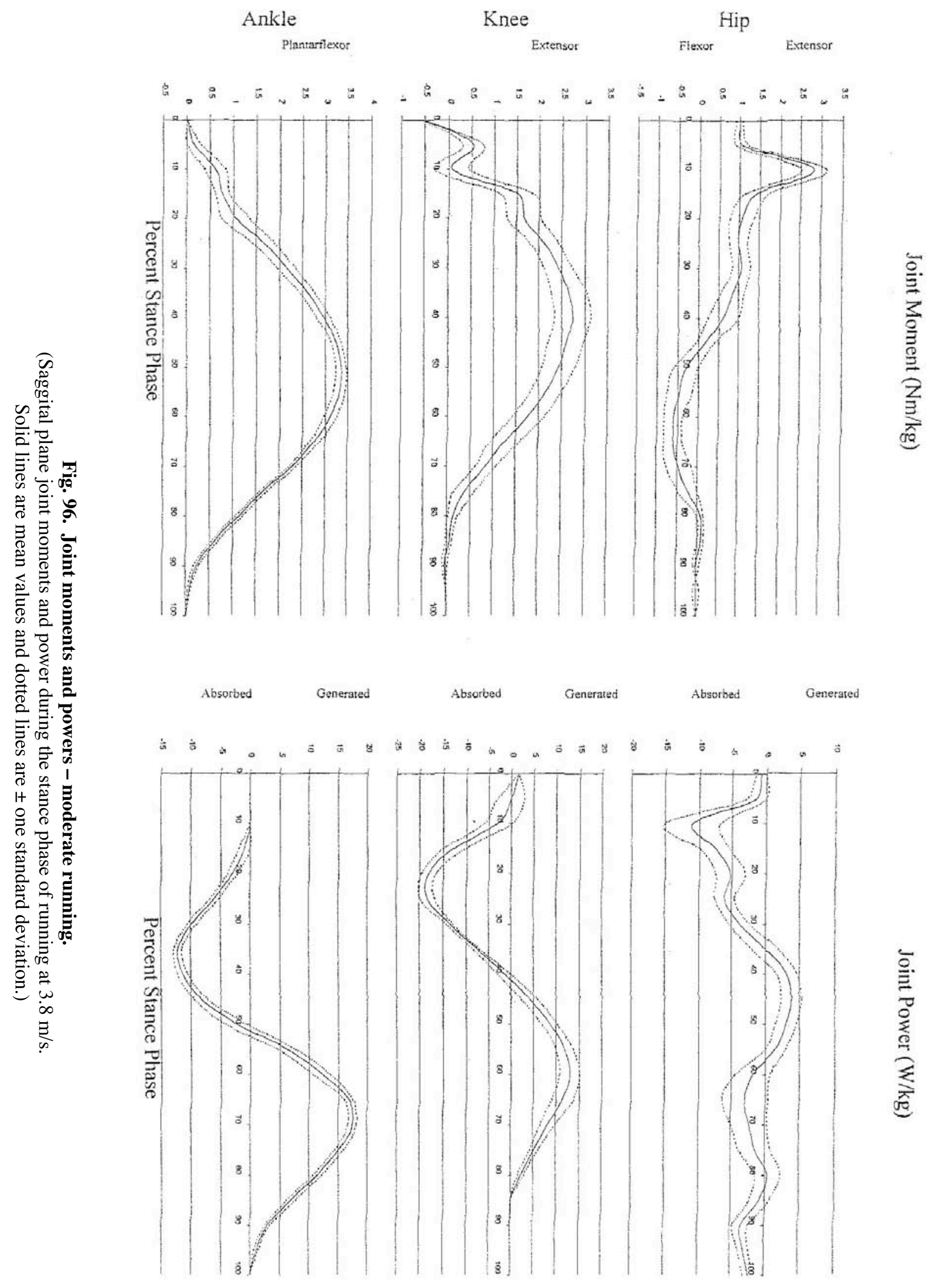



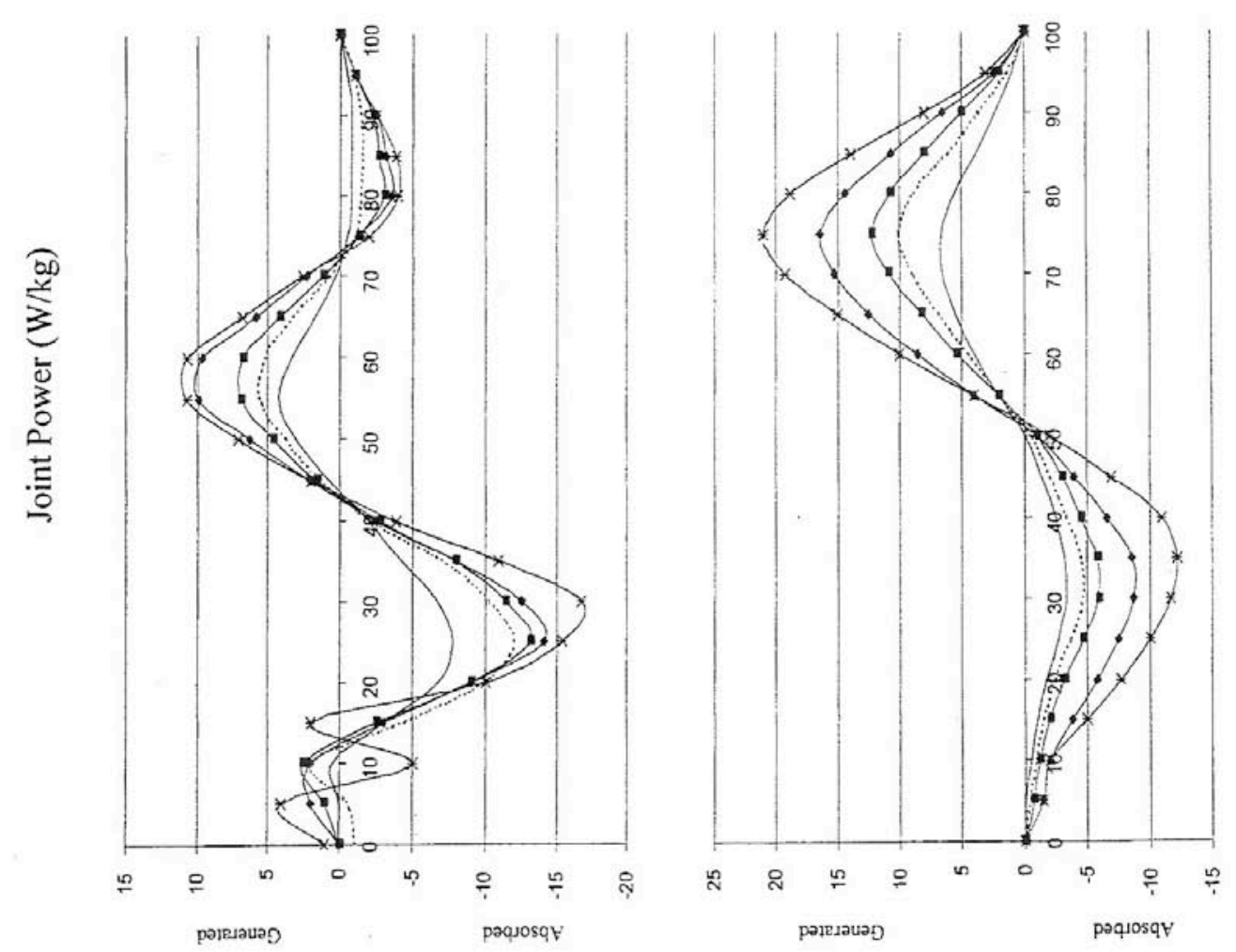

号
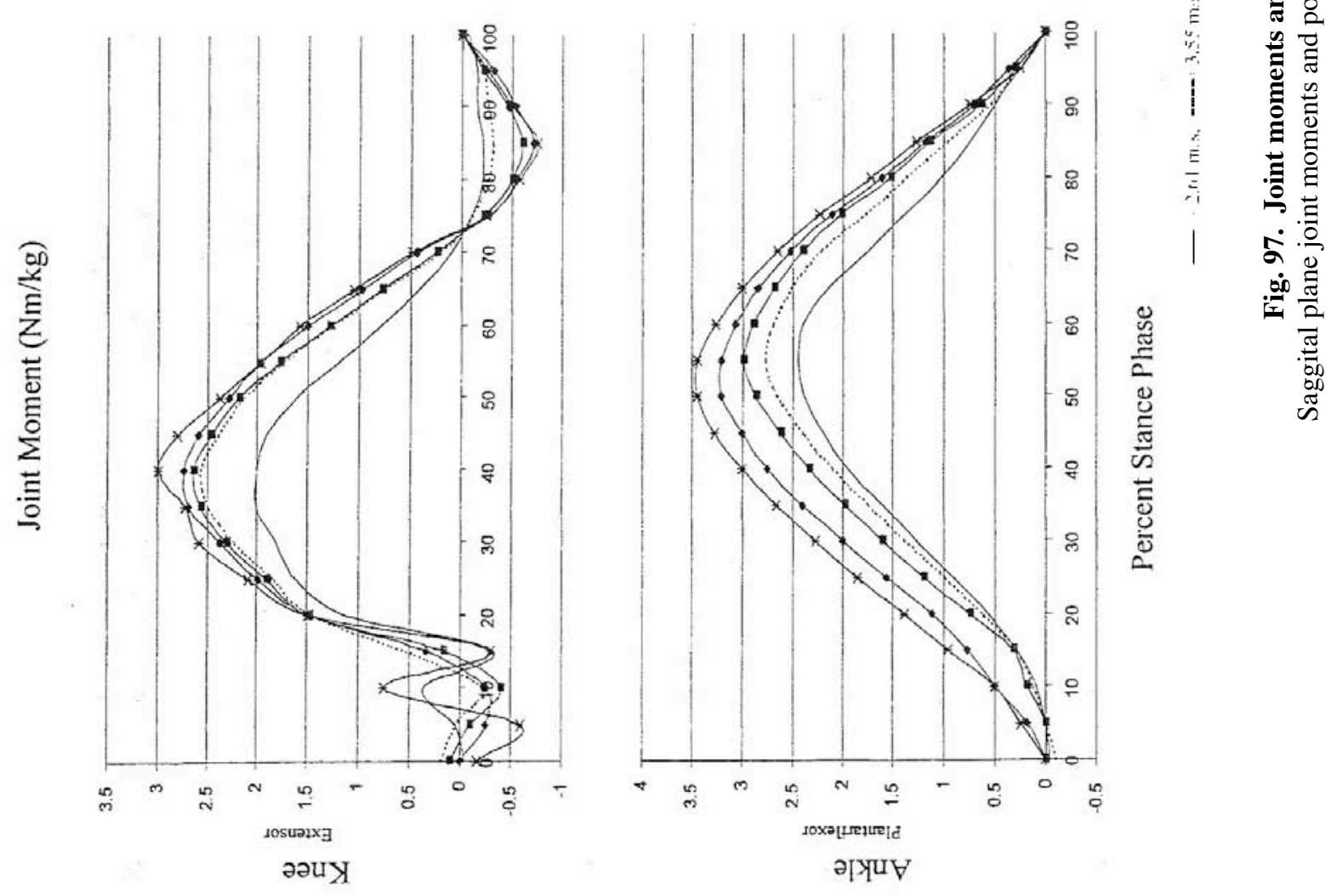


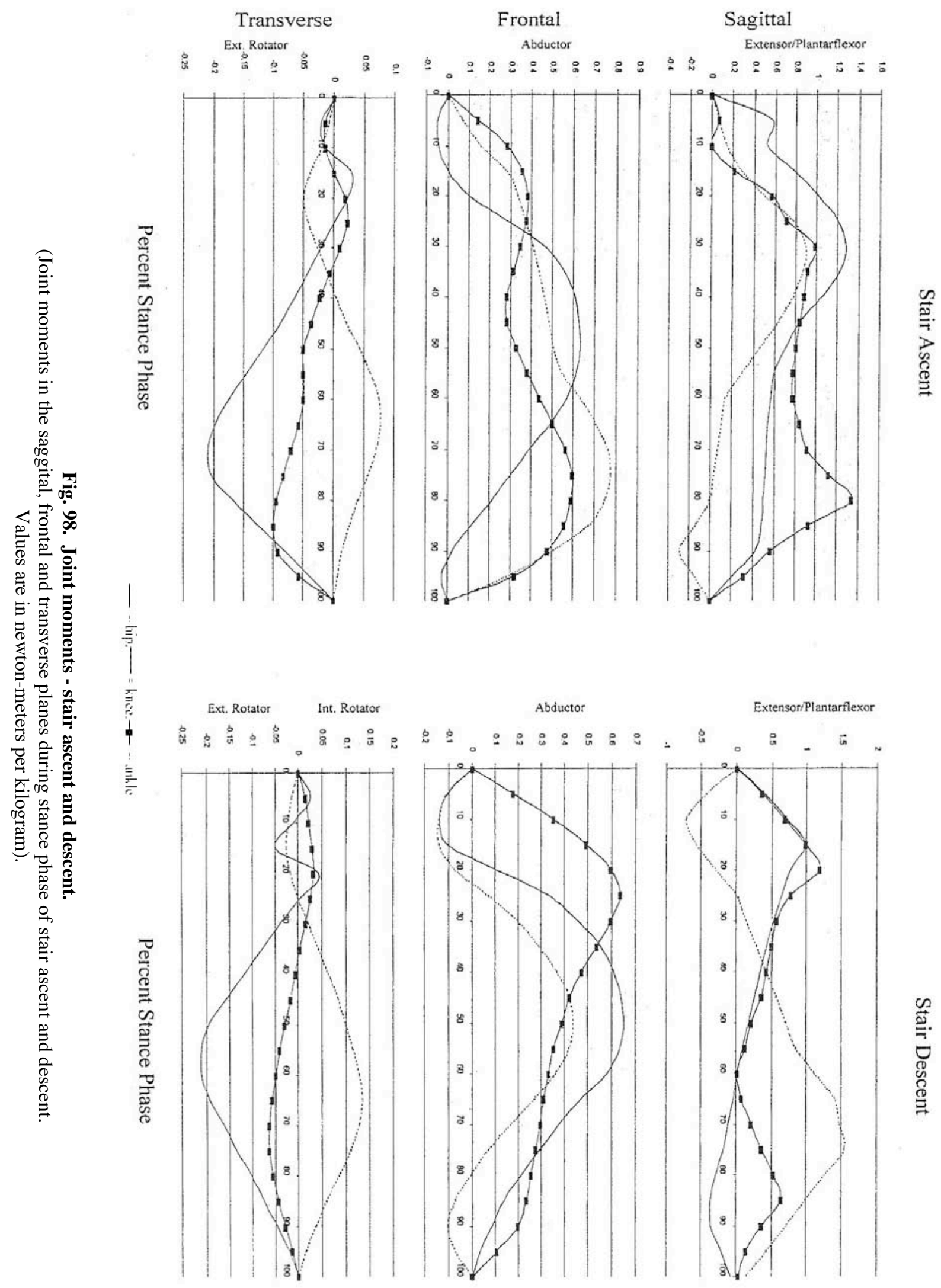



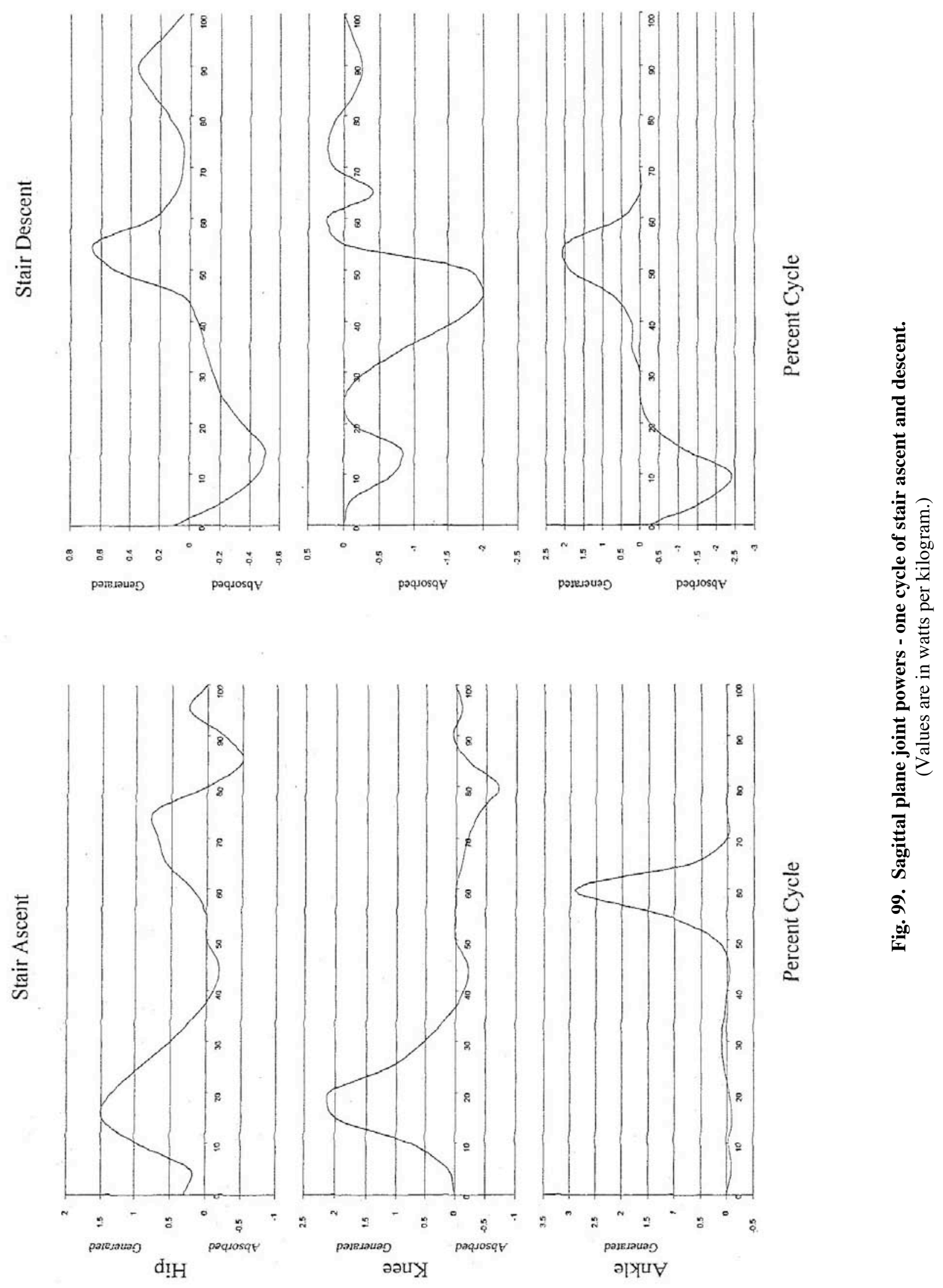


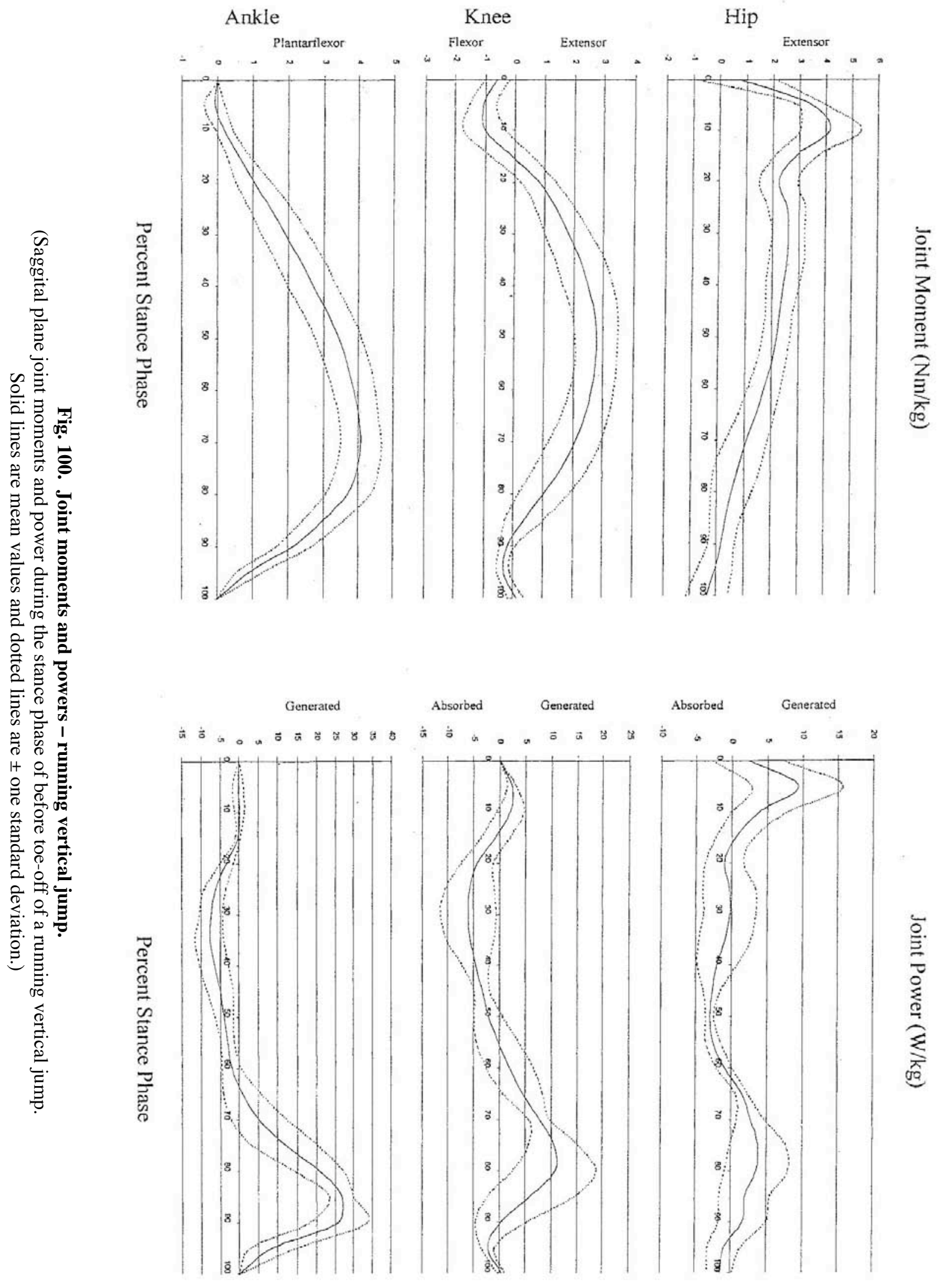


APPENDIX D

SOLE THICKNESS TEST DATA 
This appendix contains the data from the human factors analysis on the effects of sole thickness of the boot interface. This study was performed by ARL and is documented in Boynton and Crowell, 2003.

Table 7. Biomechanics variables for walking

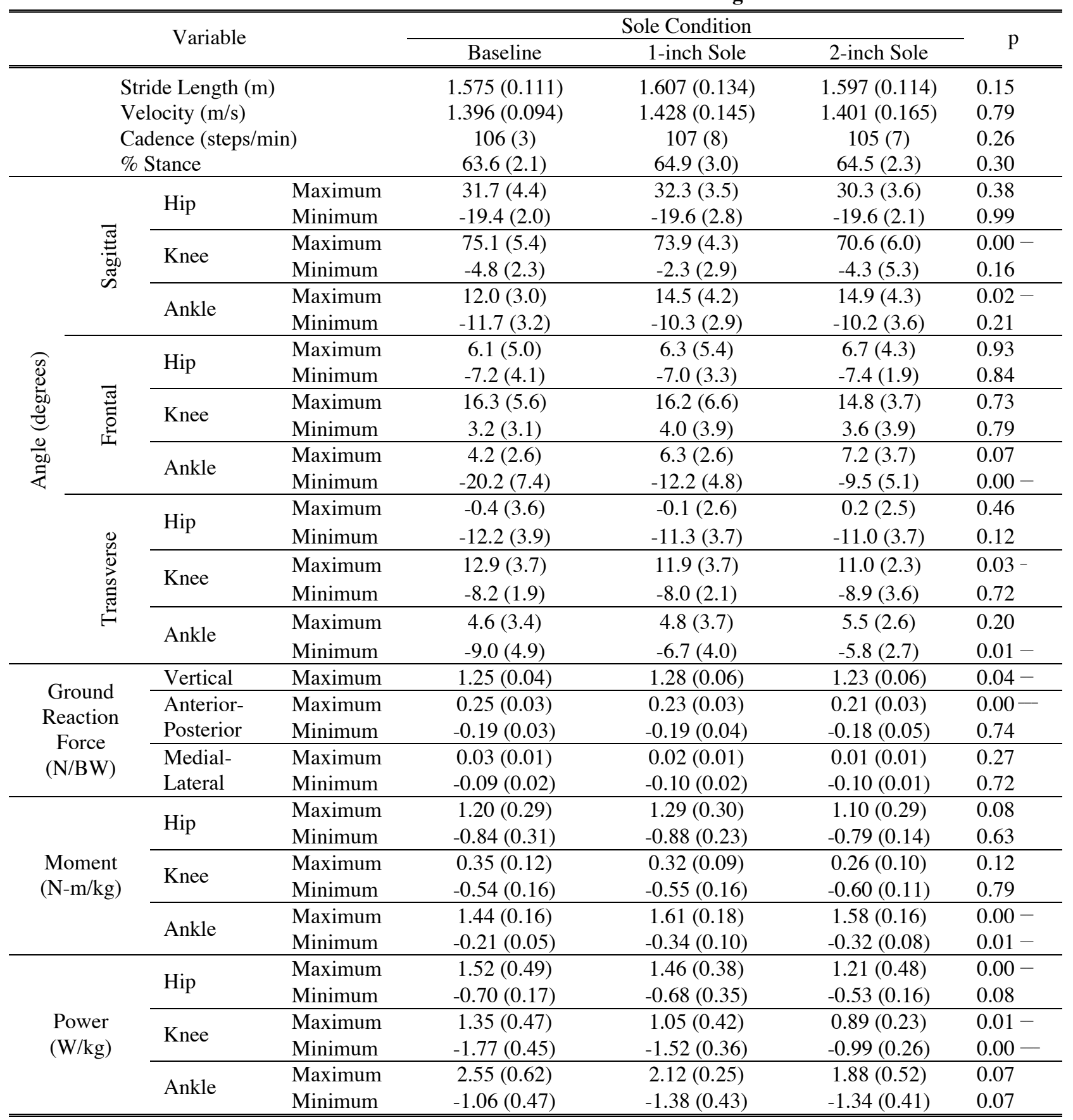

Note: Values given as mean (s.d.), $\mathrm{N}=12$

Positive values indicate flexion, adduction, and external rotation; vertical, posterior and medial GRF; extensor moment and power generation

Negative values indicate extension, abduction and internal rotation; anterior and lateral GRF; flexor moment and power absorption

statistically significant difference in Baseline and 1-inch Sole condition means (Fisher LSD, p<0.05)

statistically significant difference in Baseline and 2-inch Sole condition means (Fisher LSD, p<0.05)

- statistically significant difference in 1-inch Sole and 2-inch Sole condition means (Fisher LSD, p<0.05) 
Table 8. Biomechanics variables for running

\begin{tabular}{|c|c|c|c|c|c|c|}
\hline & Variable & & & ole Condition & & \\
\hline & variadie & & Baseline & 1-inch Sole & 2-inch Sole & $\mathrm{p}$ \\
\hline & le Length & & $2.435(0.285)$ & $2.341(0.392)$ & $2.351(0.289)$ & 0.43 \\
\hline & city $(\mathrm{m} / \mathrm{s})$ & & $3.097(0.324)$ & $3.012(0.362)$ & $3.011(0.327)$ & 0.41 \\
\hline & ence (steps & & $153(7)$ & $156(13)$ & $154(10)$ & 0.54 \\
\hline & tance & & $39.4(4.4)$ & $41.5(4.0)$ & $42.0(3.3)$ & 0.05 \\
\hline & & Maximum & $38.2(7.7)$ & $38.6(7.1)$ & $37.6(7.9)$ & 0.96 \\
\hline & Hip & Minimum & $-25.9(5.4)$ & $-24.9(3.4)$ & $-25.6(5.4)$ & 0.65 \\
\hline$\stackrel{\Xi}{\Xi}$ & Knee & Maximum & $94.0(10.9)$ & $96.8(11.7)$ & $90.3(10.7)$ & 0.29 \\
\hline שू & Knee & Minimum & $7.7(5.2)$ & $9.7(4.9)$ & $6.6(4.4)$ & 0.25 \\
\hline & & Maximum & $23.8(2.7)$ & $26.0(3.4)$ & $25.4(3.0)$ & 0.08 \\
\hline & Ankle & Minimum & $-13.6(4.2)$ & $-10.0(5.2)$ & $-11.1(4.9)$ & 0.07 \\
\hline & Hin & Maximum & $10.5(3.5)$ & $10.7(4.9)$ & $10.6(4.6)$ & 0.94 \\
\hline$\stackrel{\mathscr{J}}{0}$ & Hip & Minimum & $-5.6(3.5)$ & $-5.6(4.6)$ & $-4.5(3.3)$ & 0.80 \\
\hline 窇 & Knee & Maximum & $18.2(5.2)$ & $18.2(6.5)$ & $18.5(5.0)$ & 0.93 \\
\hline $\overrightarrow{0}$ & Knee & Minimum & $3.8(3.9)$ & $4.2(3.5)$ & $3.8(3.9)$ & 0.46 \\
\hline$\frac{0}{00}$ & Ankle & Maximum & $12.0(5.7)$ & $12.8(3.6)$ & $12.8(4.3)$ & 0.67 \\
\hline & Ankie & Minimum & $-13.4(5.1)$ & $-8.4(3.9)$ & $-8.6(3.4)$ & $0.00-$ \\
\hline & Hin & Maximum & $2.5(4.0)$ & $2.3(1.8)$ & $1.5(1.6)$ & 0.90 \\
\hline & пाр & Minimum & $-9.1(3.2)$ & $-9.0(2.9)$ & $-8.6(3.3)$ & 0.85 \\
\hline$\stackrel{\bar{D}}{D}$ & Knee & Maximum & $18.3(3.8)$ & $18.1(5.9)$ & $17.9(2.9)$ & 0.92 \\
\hline 墕 & NIIEe & Minimum & $-8.8(2.0)$ & $-8.4(2.0)$ & $-8.2(3.1)$ & 0.73 \\
\hline & Ankle & Maximum & $6.2(4.4)$ & $4.2(2.7)$ & $5.1(2.0)$ & 0.42 \\
\hline & AnкIE & Minimum & $-5.5(3.8)$ & $-5.1(3.3)$ & $-3.6(2.5)$ & 0.07 \\
\hline & Vertical & Maximum & $2.45(0.20)$ & $2.45(0.18)$ & $2.41(0.21)$ & 0.99 \\
\hline Ground & Anterior- & Maximum & $0.28(0.03)$ & $0.26(0.04)$ & $0.25(0.04)$ & 0.32 \\
\hline $\begin{array}{c}\text { Reaction } \\
\text { Force }\end{array}$ & Posterior & Minimum & $-0.23(0.06)$ & $-0.20(0.07)$ & $-0.21(0.05)$ & 0.33 \\
\hline $\begin{array}{l}\text { Force } \\
\text { (N/BW) }\end{array}$ & Medial- & Maximum & $0.00(0.01)$ & $0.01(0.01)$ & $0.01(0.01)$ & 0.31 \\
\hline & Lateral & Minimum & $-0.23(0.04)$ & $-0.24(0.04)$ & $-0.23(0.03)$ & 0.63 \\
\hline & Hin & Maximum & $2.03(0.83)$ & $1.76(0.66)$ & $1.51(0.39)$ & 0.36 \\
\hline & Hip & Minimum & $-0.71(0.17)$ & $-0.70(0.17)$ & $-0.64(0.15)$ & 0.75 \\
\hline Moment & Knee & Maximum & $1.54(0.49)$ & $1.65(0.50)$ & $1.58(0.40)$ & 0.62 \\
\hline$(\mathrm{N}-\mathrm{m} / \mathrm{kg})$ & Knee & Minimum & $-0.43(0.61)$ & $-0.43(0.20)$ & $-0.46(0.22)$ & 0.55 \\
\hline & Ankle & Maximum & $2.60(0.30)$ & $2.72(0.40)$ & $2.64(0.40)$ & 0.13 \\
\hline & Ankle & Minimum & $-0.33(0.19)$ & $-0.41(0.35)$ & $-0.20(0.07)$ & 0.13 \\
\hline & Hin & Maximum & $4.66(2.00)$ & $4.58(2.28)$ & $3.74(2.12)$ & 0.06 \\
\hline & Hip & Minimum & $-1.85(0.85)$ & $-1.47(0.78)$ & $-1.67(0.58)$ & 0.45 \\
\hline Power & Knee & Maximum & $3.77(1.63)$ & $4.25(1.56)$ & $3.50(0.85)$ & 0.40 \\
\hline$(\mathrm{W} / \mathrm{kg})$ & Knee & Minimum & $-5.30(1.56)$ & $-5.57(1.78)$ & $-4.62(1.60)$ & 0.43 \\
\hline & Ankle & Maximum & $8.16(0.97)$ & $7.75(1.13)$ & $7.51(1.30)$ & 0.35 \\
\hline & Ankie & Minimum & $-7.19(0.79)$ & $-7.24(1.55)$ & $-7.17(1.47)$ & 0.71 \\
\hline
\end{tabular}

Note: Values given as mean (s.d.), $\mathrm{N}=12$

Positive values indicate flexion, adduction, and external rotation; vertical, posterior and medial GRF; extensor moment and power generation

Negative values indicate extension, abduction and internal rotation; anterior and lateral GRF; flexor moment and power absorption

- statistically significant difference in Baseline and 1-inch Sole condition means (Fisher LSD, p<0.05)

statistically significant difference in Baseline and 2-inch Sole condition means (Fisher LSD, p<0.05)

- statistically significant difference in 1 -inch Sole and 2-inch Sole condition means (Fisher LSD, $<<0.05$ ) 
Table 9. Biomechanics variables for squatting

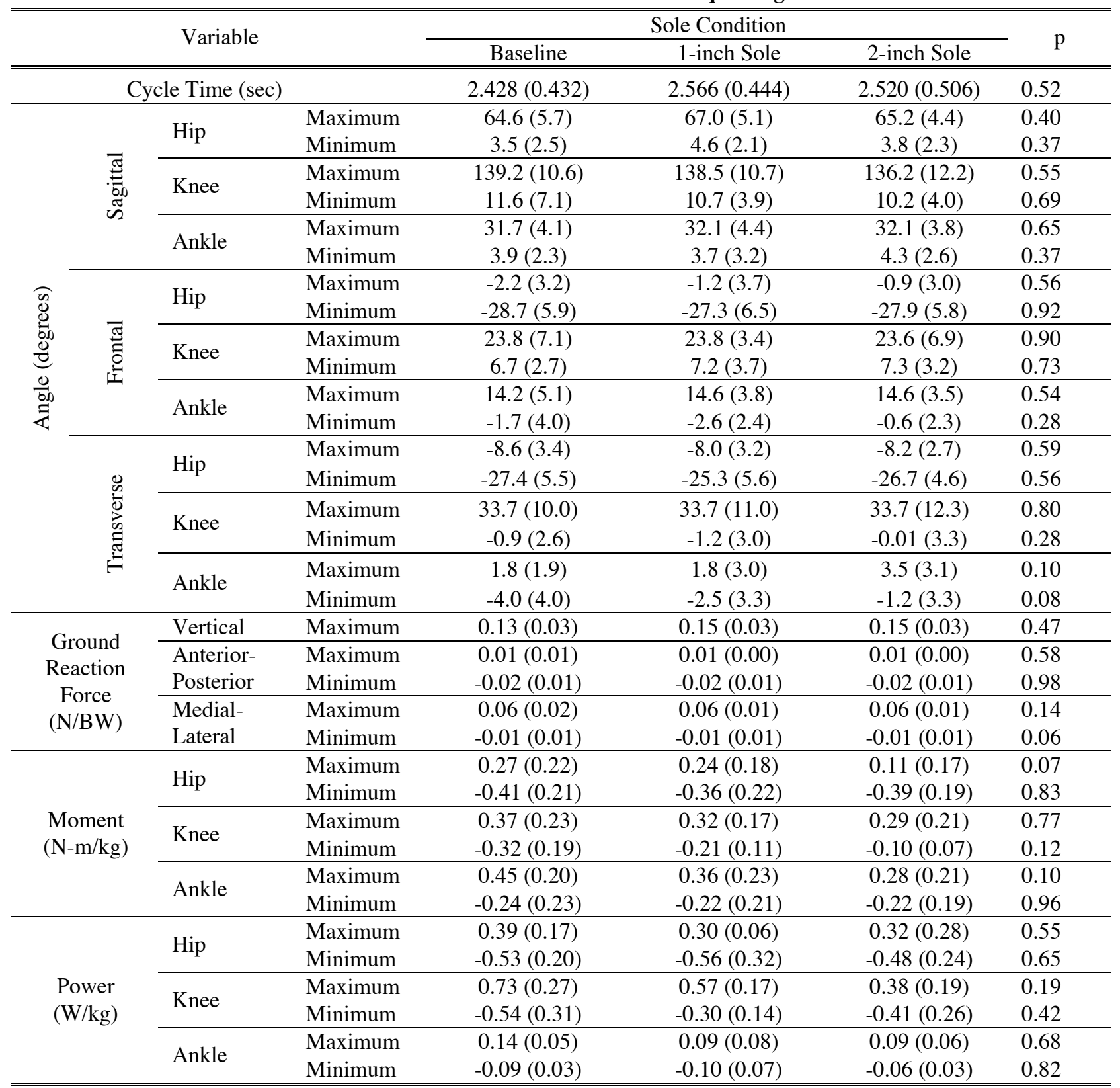

Note: Values given as mean (s.d.), $\mathrm{N}=12$

Positive values indicate flexion, adduction, and external rotation; vertical, posterior and medial GRF; extensor moment and power generation Negative values indicate extension, abduction and internal rotation; anterior and lateral GRF; flexor moment and power absorption

- statistically significant difference in Baseline and 1-inch Sole condition means (Fisher LSD, $\mathrm{p}<0.05$ )

- statistically significant difference in Baseline and 2-inch Sole condition means (Fisher LSD, $p<0.05$ )

- statistically significant difference in 1-inch Sole and 2-inch Sole condition means (Fisher LSD, p<0.05) 
Table 10. Biomechanics variables for right kneeling

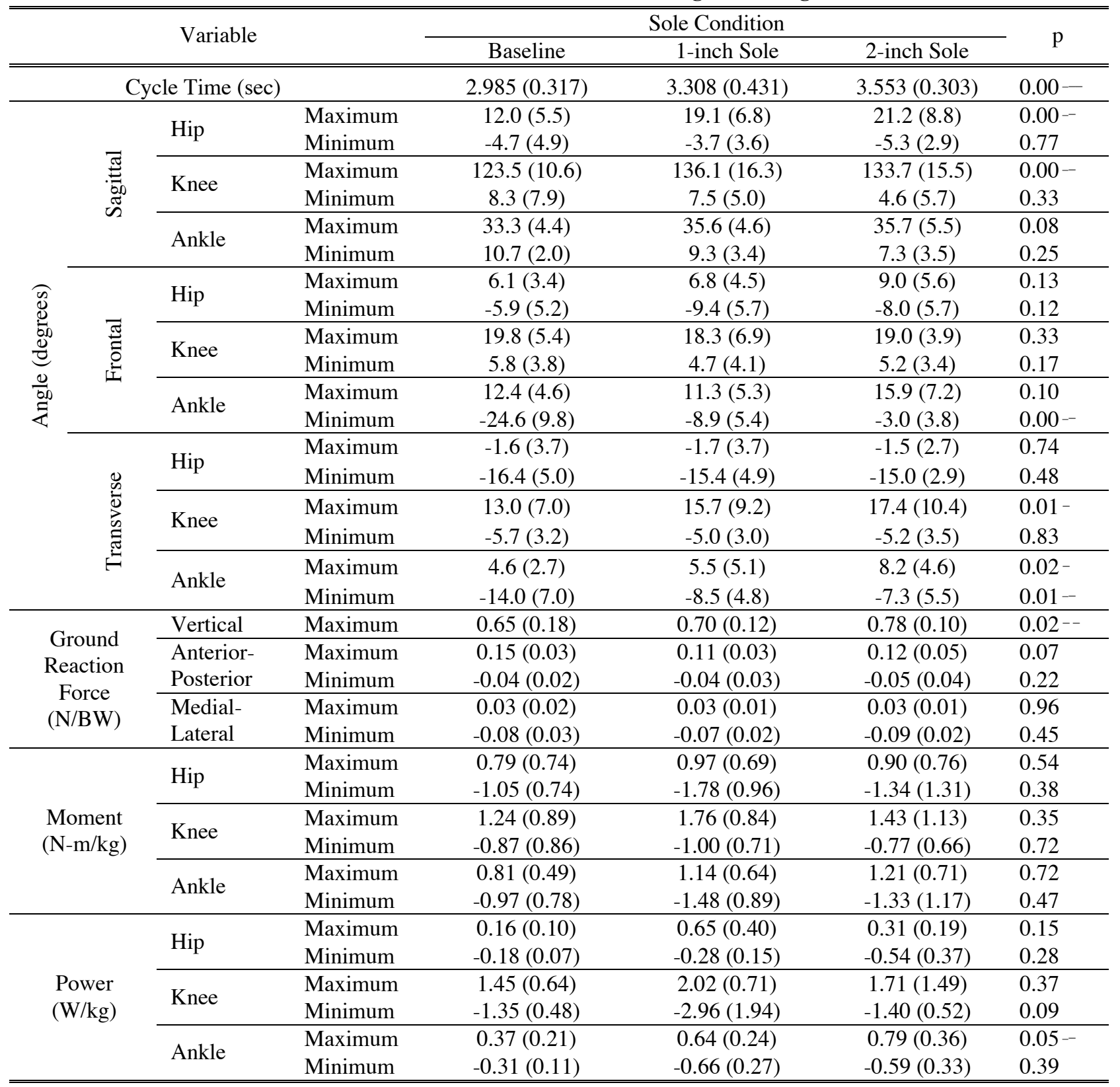

Note: Values given as mean (s.d.), $\mathrm{N}=12$

Positive values indicate flexion, adduction, and external rotation; vertical, posterior and medial GRF; extensor moment and power generation Negative values indicate extension, abduction and internal rotation; anterior and lateral GRF; flexor moment and power absorption

- statistically significant difference in Baseline and 1-inch Sole condition means (Fisher LSD, $\mathrm{p}<0.05$ )

- statistically significant difference in Baseline and 2-inch Sole condition means (Fisher LSD, $p<0.05$ )

- statistically significant difference in 1-inch Sole and 2-inch Sole condition means (Fisher LSD, p<0.05) 
Table 11. Biomechanics variables for left kneeling

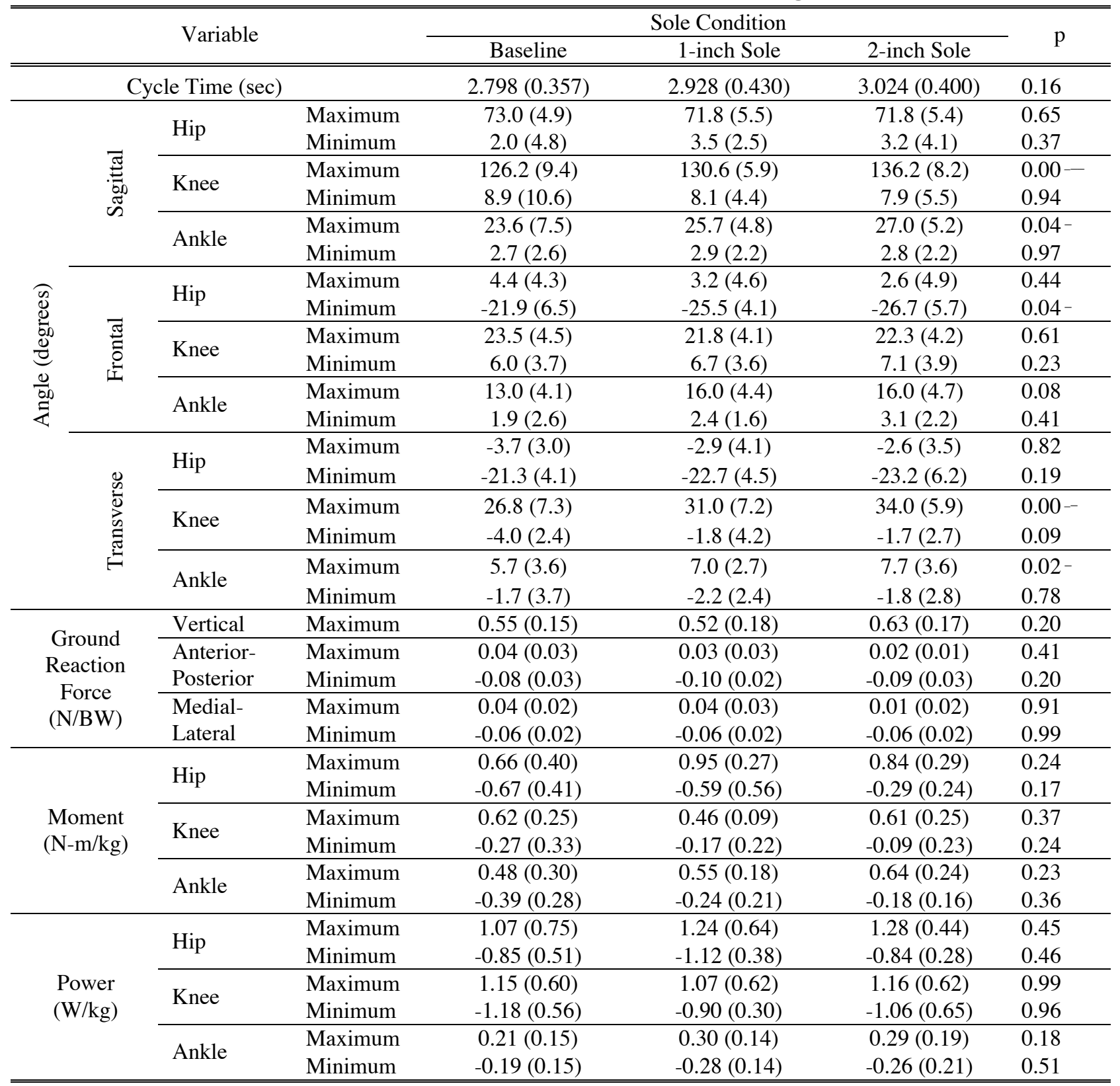

Note: Values given as mean (s.d.), $\mathrm{N}=12$

Positive values indicate flexion, adduction, and external rotation; vertical, posterior and medial GRF; extensor moment and power generation Negative values indicate extension, abduction and internal rotation; anterior and lateral GRF; flexor moment and power absorption

- statistically significant difference in Baseline and 1-inch Sole condition means (Fisher LSD, $\mathrm{p}<0.05$ )

- statistically significant difference in Baseline and 2-inch Sole condition means (Fisher LSD, $p<0.05$ )

- statistically significant difference in 1-inch Sole and 2-inch Sole condition means (Fisher LSD, p<0.05) 
Table 12. Obstacle completion times (seconds)

\begin{tabular}{|c|c|c|c|c|}
\hline \multirow{2}{*}{ Obstacle } & \multicolumn{3}{|c|}{ Sole Condition } & \multirow{2}{*}{$\mathrm{p}$} \\
\hline & Baseline & 1-inch Sole & 2-inch Sole & \\
\hline Log Balance & $12.7(3.0)$ & $15.4(3.4)$ & $16.0(4.4)$ & $0.01-$ \\
\hline Up \& Down & $5.4(1.0)$ & $6.0(2.2)$ & $6.4(3.0)$ & 0.13 \\
\hline Tires & $7.8(0.9)$ & $8.3(1.3)$ & $8.7(1.4)$ & $0.02-$ \\
\hline House & $9.3(0.9)$ & $9.5(1.2)$ & $9.9(1.8)$ & 0.07 \\
\hline Zig-Zag & $5.9(0.7)$ & $5.9(0.7)$ & $6.3(0.9)$ & 0.12 \\
\hline
\end{tabular}

Note: $\quad$ values given as mean (s.d.), $\mathrm{N}=12$

- statistically significant difference in Baseline and 1-inch Sole condition means (Fisher LSD, p<0.05)

- statistically significant difference in Baseline and 2-inch Sole condition means (Fisher LSD, $p<0.05$ )

- statistically significant difference in 1-inch Sole and 2-inch Sole condition means (Fisher LSD, p<0.05)

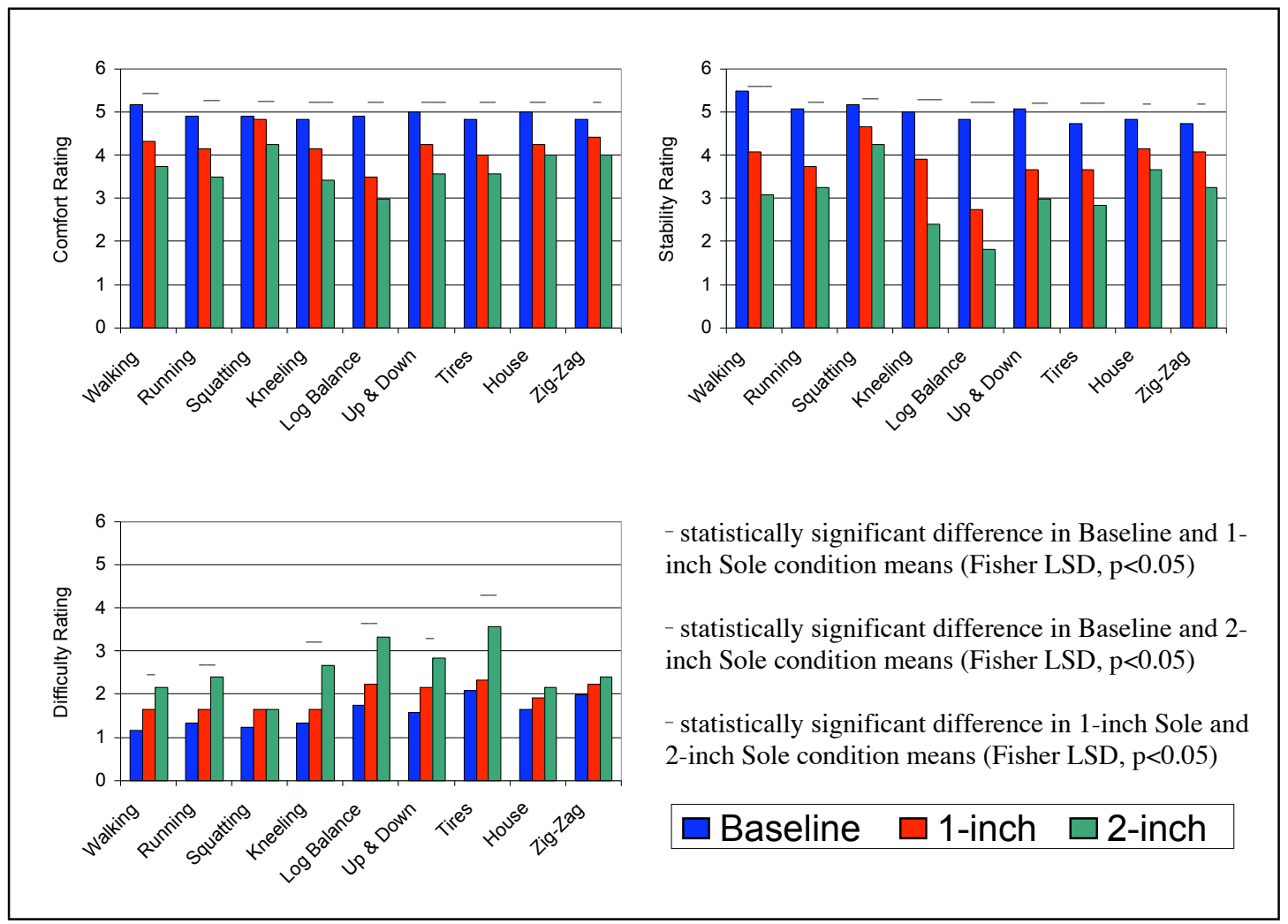

Fig. 101. Mean comfort, stability and difficulty questionnaire responses $(\mathrm{N}=12)$. 


\section{APPENDIX E \\ FEASIBILITY STUDY - POWER SOURCE COMPARISON}


This appendix is an excerpt from an ORNL exoskeleton feasibility study conducted for DARPA in 2000 (Jansen et al., 2000). This appendix provides some of the background and basis for the design approach taken and is included here for completeness and the convenience of the reader.

Issues relating to potential power sources and both steady-state and transient power requirements will be addressed. While no single power source can meet all the requirements optimally, a hybrid system that can satisfy both steady-state and transient power needs appears to be the most promising approach.

\section{POWER AND ENERGY REQUIREMENTS}

To get a rough idea as to the power requirement of an exoskeleton, it is instructive to look at the power requirements of man-made and living vehicles (i.e., animals). Gabrielli and von Karman (Gabrielli, 1950) showed that specific resistance, $\square$, is an accurate measure of power requirement for a given velocity for a wide range of different types of mechanical locomotion including living organisms. Specific resistance is defined (using the same units used by Gabrielli) as

$$
\mathrm{D}=\frac{\mathrm{P}}{\mathrm{W} \cdot \mathrm{V}}
$$

where $\mathrm{P}=$ power in units of $\mathrm{lb}-\mathrm{ft} / \mathrm{s}, \mathrm{W}=$ weight in $\mathrm{lb}$, and $\mathrm{V}=$ speed in $\mathrm{ft} / \mathrm{s}$. For a human being walking on level ground, the specific resistance is around 0.06 to 0.09 and increases to 0.12 when running. Bicycles, cars, and horses at low velocities have a specific resistance ranging from 0.01 to 0.05 . Since the specific resistance of an exoskeleton is not known, we will assume a conservative range from 0.01 to 0.1 . If the exoskeleton and its payload is $\mathrm{W}_{\text {ex }}$ then the power requirement, $\mathrm{P}_{\mathrm{ex}}$, of such a machine must range from $\mathrm{P}_{\mathrm{ex}}=(.01 \text { to } 0.1)^{*} \mathrm{~W}_{\mathrm{ex}} \mathrm{V}_{\mathrm{ex}}$ where $\mathrm{V}_{\mathrm{ex}}$ is the velocity of the exoskeleton. At a speed of $2 \mathrm{~m} / \mathrm{s}$ (or $4.5 \mathrm{mph}$ or $6.6 \mathrm{ft} / \mathrm{s}$ ), $\mathrm{P}_{\mathrm{ex}}$ would range from $6.6 \mathrm{lb}-\mathrm{ft} / \mathrm{s}$ to $66 \mathrm{lb}-\mathrm{ft} / \mathrm{s}$ per $100 \mathrm{lb}$ of load (or 8.9 to $89 \mathrm{~W}$ per $100 \mathrm{lb}$ of load). For a $350 \mathrm{lb}$ total load (exoskeleton and payload), the upper power requirement is around $310 \mathrm{~W}$. Standard earthmoving equipment has a payload to weight ratio of $1: 2$, which implies a conventional hydraulic system and would suggest an exoskeleton weight of approximately $233 \mathrm{lb}$ and a payload of $117 \mathrm{lb}$. Based on anticipated performance improvements of an exoskeleton in comparison to heavy equipment, the payload to weight ratio is expected to be significantly better.

Two cases will be compared, walking while lifting $100 \mathrm{lb}$ and running. If in addition to walking, the power required to lift $100 \mathrm{lb}$ vertically against gravity at $1 \mathrm{ft} / \mathrm{s}$ is added, the additional power is around $140 \mathrm{~W}$. Thus, for an exoskeleton with maximum payload, the total power required could be expected to around $440 \mathrm{~W}$, assuming a worst-case scenario and extrapolating the same power requirements as those for a human. The second case is running. As mentioned above, about $310 \mathrm{~W}$ were calculated assuming a speed of $2 \mathrm{~m} / \mathrm{s}(4.5 \mathrm{mph})$. Increasing the speed to 3 $\mathrm{m} / \mathrm{s}(6.7 \mathrm{mph})$ and the specific resistance range to 0.01 to 0.12 for running, similarly, gives an estimate of approximately $560 \mathrm{~W}$. Based on these two scenarios, $600 \mathrm{~W}$ should be a conservative upper bound for the required steady-state power. Peak power demands could be significantly larger than $600 \mathrm{~W}$ and would affect the overall sizing of the power supply, unless temporary energy storage is used to offset peak power demands. For motions such as rapid, deep, knee bends, or climbing stairs, peak power demands could reach as high as $2 \mathrm{~kW}$. It is clear that an exoskeleton power source will have to have both large energy (600 W times operational hours) and peak-power ( $2 \mathrm{~kW},>3$ times the nominal load) capacities. 


\section{POWER SOURCES}

As discussed above, there could be significant differences in the steady-state power requirements and the peak power or transient demands. The power source must, therefore, be able to meet both the energy requirements and peak power demands and comparison of potential power sources must be made with respect to meeting both of these requirements. For an exoskeleton type of system, the specific power (power $\div$ weight) and specific energy (energy $\div$ weight) are the parameters that are more useful for comparison of potential power systems. Ragone Diagrams (Fig. 102), which plot specific energy vs specific power, provides a means for making broad, general, comparisons between potential power sources. However, it should be noted that Ragone Diagrams provide information on steady-state specific power and specific energy, but they do not provide any indication as to the response time of a power source and thus, its ability to follow demand.

Figure 102 provides a general comparison of typical fuel cells, batteries, internal combustion engines, and super capacitors. It should be noted that on-going research is continuing to expand the capabilities of these power sources. Although other power sources and storage devices, (e.g., compressed gas, thermoelectric devices, and flywheels) are available, they are not considered practical for a remote mobile device, such as an exoskeleton. From Fig. 102, it can be seen that internal combustion engines have relatively high specific power and specific energy and a hybrid system such as a fuel cell and either batteries or super capacitors can also meet the requirement of high specific power and energy. Using a single power source requires sizing the source such that it can meet both steady-state energy needs and peak power loads. For systems such as the exoskeleton, where peak loads can be several times the steady-state loads, this may result in an oversized source (both weight and cube) and a significant reduction in efficiency. Alternatively, a hybrid system can handle large variations in steady-state and peak loads, as one source is sized to provide for power peaks and another to provide steady-state energy. A hybrid system offers the possibility of a more efficient system, optimized for both peak power and steady-state energy needs at the cost of increased complexity. In the following section, potential power sources will be discussed with regard to the exoskeleton specific requirements.

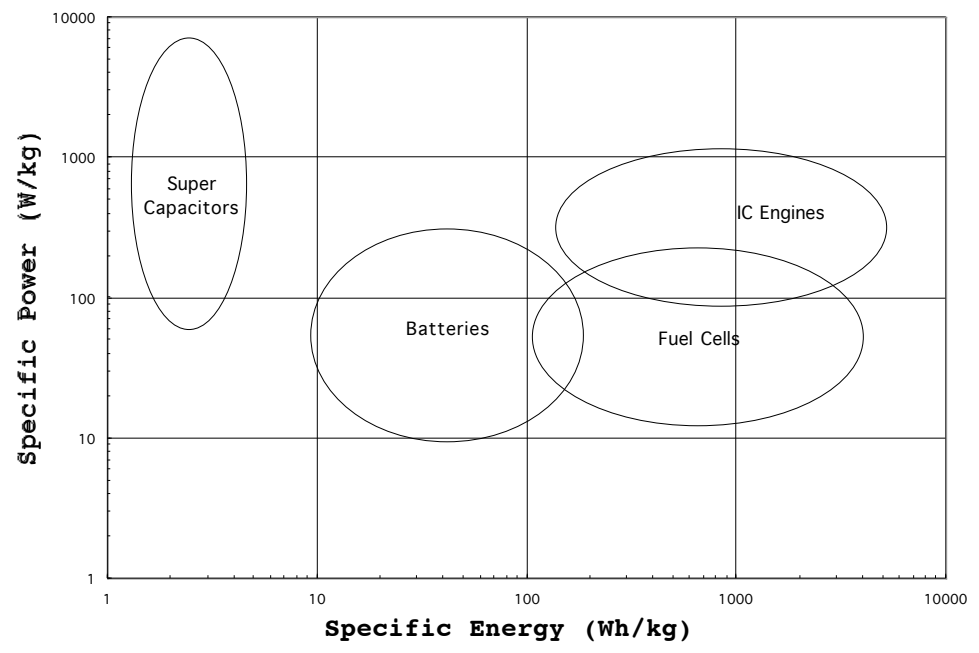

Fig. 102. Comparison of different power sources. 


\section{Fuel Cells}

The National Aeronautics and Space Administration (NASA) demonstrated the potential of fuel cells, originally discovered in 1839 , by using them to provide power during space flights in the 1960s. Since then, research has been ongoing with a focus on improving efficiency and economic viability. There are multiple types of fuel cells, typically designated by the electrolyte type, currently in various stages of development (Table 13). These include polymer electrolyte membrane (PEM), alkaline (AFC), phosphoric acid (PAFC), molten carbonate (MCFC), and solid oxide (SOFC). Each has different characteristics and is suited to particular applications. MCFCs and SOFCs operate between $600^{\circ} \mathrm{C}$ and $1000^{\circ} \mathrm{C}$ and thus, are not suitable for applications that require close proximity to a human operator. PAFCs, which use concentrated phosphoric acid (up to 100\%) for the electrolyte, can operate in temperature ranges from 150 to $220^{\circ} \mathrm{C}$, but are poor ionic conductors, susceptible to carbon monoxide (CO) poisoning at the lower temperature range, and also have low power to weight ratios (Hirschenhofer, 1999). AFCs, which operate in the 90 to $100{ }^{\circ} \mathrm{C}$ range, have been limited to military and space applications due to the expense of removing carbon dioxide $\left(\mathrm{CO}_{2}\right)$ from the fuel and air streams and are generally being phased out (Thomas, 1999). PEM fuel cells have a relatively low operating temperature (about $80^{\circ} \mathrm{C}$ ) and are the most appropriate for portable applications in close proximity to an operator.

Table 13. Comparison of fuel cell types

(Sources: Hirschenhofer, 1999 and Thomas, 1999.)

\begin{tabular}{|l|l|c|l|}
\hline Fuel Cell Type & \multicolumn{1}{|c|}{ Electrolyte } & $\begin{array}{c}\text { Operating } \\
\text { Temperature }\left({ }^{\circ} \mathrm{C}\right)\end{array}$ & \multicolumn{1}{|c|}{ Comments } \\
\hline Alkaline (AFC) & $\begin{array}{l}\text { Aqueous solution of potassium } \\
\text { hydroxide soaked in a matrix }\end{array}$ & $90-100$ & $\begin{array}{l}\text { Expensive removal of } \mathrm{CO}_{2} \\
\text { required. High performance. }\end{array}$ \\
\hline $\begin{array}{l}\text { Phosphoric Acid } \\
(\text { PAFC) }\end{array}$ & $\begin{array}{l}\text { Immobilized liquid phosphoric } \\
\text { acid }\end{array}$ & $175-205$ & $\begin{array}{l}\text { Low power/weight and } \\
\text { power/vol. High efficiency. } \\
\text { Tolerant of fuel impurities. }\end{array}$ \\
\hline $\begin{array}{l}\text { Polymer } \\
\text { Electrolyte } \\
\text { Membrane (PEM) }\end{array}$ & Ion exchange membrane & $60-100$ & $\begin{array}{l}\text { Low temperatures. Quick } \\
\text { startup. Sensitive to fuel } \\
\text { impurities. }\end{array}$ \\
\hline $\begin{array}{l}\text { Molten Carbonate } \\
(\text { MCFC) }\end{array}$ & $\begin{array}{l}\text { Immobilized liquid molten } \\
\text { carbonate }\end{array}$ & $600-1000$ & $\begin{array}{l}\text { High temperatures lead to } \\
\text { corrosion and cell breakdown. } \\
\text { High temperature results in } \\
\text { higher efficiency, inexpensive } \\
\text { catalysts, more fuel options. }\end{array}$ \\
\hline $\begin{array}{l}\text { Solid Oxide } \\
\text { (SOFC) }\end{array}$ & $\begin{array}{l}\text { Ceramic (zirconium oxide } \\
\text { based) }\end{array}$ & $800-1000$ \\
\hline
\end{tabular}

While ICEs have superior steady-state specific power, fuel cells are more human-compatible when noise and waste by-products are considered. In particular, PEM fuel cells operate at low temperatures with water as the waste by-product. The PEM fuel cells, however, require careful water management and a particularly clean source of hydrogen (essentially $\mathrm{CO}$ and $\mathrm{CO}_{2}$ free). These fuel cells are susceptible to $\mathrm{CO}$ poisoning, which adversely affects the fuel cell voltage, especially at higher current densities (Hirschenhofer, 1999). A major focus of research and development with respect to fuel cells is related to reformer technology, which is being developed to extract hydrogen from hydrocarbon fuels. Significant progress has been made in research concerned with direct methanol PEM fuel cells, which use the methanol directly without 
going through a reformer. However, the power density of direct methanol PEMs still lags that of hydrogen/air PEMs due to the need for significantly more of the expensive platinum catalyst (in comparison to hydrogen/air PEMs), and the loss of performance caused by methanol "crossover" (Thomas, 1999). A potential clean source of hydrogen for a PEM is chemical reactions, which are similar to commercially available systems based on a single exothermic sodium hydroxide reaction. The water generated at the fuel cell reaction can be recycled for use in the hydrogen generation, to reduce weight and further increase efficiency.

Commercially available fuel cells in the $500 \mathrm{~W}$ to $2 \mathrm{~kW}$ range can have specific powers on the order of 50 to $150 \mathrm{~W} / \mathrm{kg}$ (Ballard and Analytic Power Corporation). AeroVironment, Inc., has developed custom fuel cells for remote power applications in the same range, with specific powers of $675 \mathrm{~W} / \mathrm{kg}$ for the cells only and on the order of $225 \mathrm{~W} / \mathrm{kg}$ including ancillary support equipment. The specific power is comparable to that for batteries and below that for super capacitors and internal combustion engines. The overall specific energy of the fuel cell system is highly dependent upon the generating system for hydrogen, which has a specific energy (higher heating value) of $40 \mathrm{kWh} / \mathrm{kg}$.

Chemical reactions as a means to generate a suitable supply of hydrogen for a fuel cell have been examined. Three types of hydrogen-releasing chemical processes have been evaluated as means for feeding a fuel cell that would power an exoskeleton. These processes are the reaction of pure elements (alkali metals, alkaline earth elements, etc.) with water, reaction of alkali and alkaline earth hydrides with water, and the decomposition of metal hydrides. The methods considered are summarized and compared and the relative advantages and disadvantages of each with regard to the proposed application discussed.

\section{Alkali and Alkaline Earth/Water Reactions}

The reactions of alkali elements (specifically lithium, sodium, and potassium) with water are rapid, irreversible, and highly exothermic. The general form of the reaction is

$$
2 \mathrm{M}+2 \mathrm{H}_{2} \mathrm{O} \square 2 \mathrm{MOH}+\mathrm{H}_{2} .
$$

As the reactions are irreversible, the rate cannot be altered by controlling the concentration (or the pressure) of the products. The rate of hydrogen generation may be controlled by the rate at which either or both of the reactants are added to the reaction vessel. However, controlling alkali-water reactions by controlling the rate of water addition is impracticable because the amount of heat generated per mass of water added is more than sufficient to increase the temperature of the product to the boiling point of the hydroxide. It is preferable to control hydrogen generation by limiting the rate at which the alkali is introduced to (or comes into contact with) water. Because of the reactivity of alkali elements with water, it is essential that these alkalis be kept in a moisture-free environment before their use. Because of their inflammability, it is also essential that the heat generated by the reaction be removed efficiently.

The primary advantage of alkali-water reactions for hydrogen generation is the relatively low mass of reactants required per mass of hydrogen released, particularly when lithium and water are reacted. Much of this advantage is lost if a large excess of water is needed to effect heat removal from the process. Other advantages of alkali-water reactions are the complete conversion of reactants and a high rate of reaction. In addition to exothermicity, the 
disadvantages of these reactions include chemical instability of the reactants, difficulty in regenerating the reactants for reuse, and formation of a corrosive (caustic) by-product.

In addition to alkali elements, reactions of alkaline earth elements (e.g., calcium, magnesium) with water have been considered. These reactions take place less rapidly than do alkali-water reactions and they produce somewhat less corrosive bases. However, the mass of reactants required per mass of hydrogen produced is increased over the alkali-water case, because of the need for two molecules of water per atom of alkaline earth element. As with alkali/water reactions, alkaline earth/water reactions are highly exothermic (Table 14).

Table 14. Comparison of hydrogen-generating chemical reactions

\begin{tabular}{|c|c|c|c|}
\hline Reaction & $\begin{array}{l}\square \mathbf{H}_{\mathrm{rxn}, 298 K} \\
\text { kcal }\end{array}$ & $\begin{array}{r}\square \mathrm{H}_{\mathrm{rxn}, 298 \mathrm{~K}} \\
\mathrm{kcal} / \mathrm{gmol} \mathbf{H}_{2}\end{array}$ & $\begin{array}{l}\text { Mass Ratio } \\
\text { Reactants: } \mathrm{H}_{2}\end{array}$ \\
\hline $2 \mathrm{Na}+2 \mathrm{H}_{2} \mathrm{O} \square 2 \mathrm{NaOH}+\mathrm{H}_{2}$ & -87.78 & -87.78 & 40.7 \\
\hline $2 \mathrm{Li}+2 \mathrm{H}_{2} \mathrm{O} \square 2 \mathrm{LiOH}+\mathrm{H}_{2}$ & -106.38 & -106.38 & 24.8 \\
\hline $\mathrm{Ca}+2 \mathrm{H}_{2} \mathrm{O} \square \mathrm{Ca}(\mathrm{OH})_{2}+\mathrm{H}_{2}$ & -103.04 & -103.04 & 37.8 \\
\hline $\mathrm{NaH}+\mathrm{H}_{2} \mathrm{O} \square \mathrm{NaOH}+\mathrm{H}_{2}$ & -30.22 & -30.22 & 20.8 \\
\hline $\mathrm{LiH}+\mathrm{H}_{2} \mathrm{O} \square \mathrm{LiOH}+\mathrm{H}_{2}$ & -31.65 & -31.65 & 12.9 \\
\hline $\mathrm{CaH}_{2}+2 \mathrm{H}_{2} \mathrm{O} \square \mathrm{Ca}(\mathrm{OH})_{2}+2 \mathrm{H}_{2}$ & -57.94 & -28.97 & 19.4 \\
\hline $\mathrm{B}_{2} \mathrm{H}_{6}+6 \mathrm{H}_{2} \mathrm{O} \square 2 \mathrm{~B}(\mathrm{OH})_{3}+6 \mathrm{H}_{2}$ & -107.98 & -18.00 & 11.2 \\
\hline $\mathrm{NaBH}_{4}(a q)+2 \mathrm{H}_{2} \mathrm{O} \square \mathrm{NaBO}_{2}(a q)+4 \mathrm{H}_{2}$ & $\begin{array}{l}\text { Data not }{ }^{a} \\
\text { available }\end{array}$ & $\begin{array}{l}\text { Data not } \\
\text { available }\end{array}$ & $18.77^{b}$ \\
\hline $\mathrm{NaBH}_{4}+2 \mathrm{H}_{2} \mathrm{O} \square \mathrm{NaBO}_{2}(a q)+4 \mathrm{H}_{2}$ & -46.68 & -11.67 & $9.16^{c}$ \\
\hline $2 \mathrm{LiH} \quad \mathrm{Li}+\mathrm{H}_{2}$ & 43.2 & 43.2 & 7.9 \\
\hline $\mathrm{CaH}_{2} \square \mathrm{Ca}+2 \mathrm{H}_{2}$ & 45.1 & 22.6 & 10.4 \\
\hline $\mathrm{TiFeH}_{1.7} \square \mathrm{TiFe}+0.85 \mathrm{H}_{2}$ & 6.5 & 7.6 & 61.5 \\
\hline
\end{tabular}

${ }^{a}$ Enthalpy data for sodium borohydride were not available for the analysis. It is expected that the heat released per g-mole of hydrogen produced will be approximately equal to that released by the hydrolysis of crystalline $\mathrm{NaBH}_{4}$.

${ }_{-}^{b}$ Mass ratio assumes that water consumed by the hydrolysis reaction is not replenished.

${ }^{-c}$ Mass ratio is based on the necessary replenishment of water consumed by the reaction.

\section{Hydrolysis of Binary Hydride and Tertiary Hydrides}

Hydrogen is generated by the reaction of binary hydrides with water according to the general reaction

$$
\mathrm{MH}_{\mathrm{x}}+\mathrm{xH}_{2} \mathrm{O} \square \mathrm{M}(\mathrm{OH})_{\mathrm{x}}+\mathrm{xH}_{2}
$$

where " $x$ " is the valence of the cation. Reactions of the hydrides of lithium, sodium, calcium, and boron have been considered for the proposed application.

As Eq. (17) indicates, the reactions of alkali and alkaline earth hydrides with water occur irreversibly, and results in the release of hydrogen and the formation of a hydroxide. Relative to the hydrolysis reactions of pure alkalis and alkaline earths, the hydrolysis reactions of binary hydride release less energy per unit of hydrogen released and requires less reactant mass per mass of hydrogen released. 
In addition to alkali and alkaline hydride reactions, the reaction of diborane $\left(\mathrm{B}_{2} \mathrm{H}_{6}\right)$ with water was evaluated. The hydrolysis of diborane has the advantages of relatively low heat generation and a low mass ratio of reactants-to-hydrogen-generated. The physical state of diborane (a gas at ambient conditions) is both an advantage and a major disadvantage. Control of hydrogen generation by diborane hydrolysis can be accomplished by metering the flow of reactant gas through a water-containing vessel. However, producing hydrogen in a compact processing system would require storage of diborane at high pressure.

The hydrolysis reaction of the tertiary hydride sodium borohydride $\left(\mathrm{NaBH}_{4}\right)$ has been considered. Millennium Cell Company (MCC) is commercializing a process in which the hydride (in aqueous solution) is hydrolyzed in the presence of a metal catalyst (e.g., ruthenium, nickel, and cobalt) (Amendola, 2000). The MCC process is based on a based on a proprietary catalyst. Initial analysis indicates that the rate of hydrogen generation from the $\mathrm{NaBH}_{4}$ process far exceeds that required to power an exoskeleton and that the rate of $\mathrm{H}_{2}$ generation decreases with increasing borate concentration. A low percentage of sodium hydroxide may be required in the aqueous feed solution to suppress slow hydrolysis before the introduction of the catalyst.

The use of an aqueous feed solution during the MCC process simplifies control of the reaction rate and provides a heat transfer medium. Using a $25 \mathrm{wt} \%$ feed solution (containing $\mathrm{NaOH}$ at $1 \mathrm{wt} \%$ ), the total mass of the initial feed solution charge required to generate $1 \mathrm{lb}$ of hydrogen is $18.77 \mathrm{lb}$. The rate of generation will not remain constant because of disproportionate (by weight) depletion of sodium borohydride and water during the reaction.

To minimize the mass of reactants, the MCC process could be modified so that the solid $\mathrm{NaBH}_{4}$ is blended with water on demand, using water recycled from the fuel cell. The effect on the rate of hydrogen generation is unknown, as is the effect on the rate control scheme. In addition, no heat sink for removal of the heat generated by the reaction is present. This may be a significant problem, as the heat released during the production of one pound of hydrogen from the solid borate is approximately equal to that required to vaporize $11 \mathrm{lb}$ of water at ambient conditions.

\section{Metal Hydride Decomposition}

Thermal decomposition of metal hydrides is the most commonly used means of hydrogen release from a chemical storage medium. Decomposition reactions are reversible and endothermic. Consequently, terminating the input of heat to the process can stop the reaction. Adjusting the rate of heat input or the hydrogen pressure can control the rate of hydrogen released over the process. Furthermore, the density of hydrogen stored on metal substrates is greater than the density of pure, liquid hydrogen.

Binary hydrides are not generally suitable for on-demand release because of their thermal stability. For example, hydrides of lithium, sodium, and calcium release hydrogen at significant pressures (i.e., $1 \mathrm{~atm}$ ) only at temperatures in excess of $300^{\circ} \mathrm{C}$. Transition metal and rare-earth alloy hydrides are better suited for hydrogen release applications since hydrogen is released effectively at temperatures slightly above ambient. As a rule of thumb, in order for hydrogen to be released at $1 \mathrm{~atm}$ pressure and at a temperature of about $27^{\circ} \mathrm{C}$, the heat of decomposition should be $9 \mathrm{kcal} / \mathrm{gmole}$ for $\mathrm{H}_{2}$. Of the hydride substrates developed for fuel cell applications, the titanium-iron substrate has undergone the most development. The hydride:hydrogen mass ratio for the $\mathrm{TiFeH}_{1.7}$ hydride is about 61 . 
From a mass conservation standpoint, the hydrolysis of $\mathrm{NaBH}_{4}$ is the most attractive option for hydrogen generation. Generation of $1 \mathrm{lb}$ of hydrogen will consume $9.16 \mathrm{lb}$ of sodium borohydride. At a hydrogen production rate of $1 \mathrm{lb} / 8 \mathrm{~h}$, heat will be generated at a rate of $1,303 \mathrm{Btu} / \mathrm{h}$.

From a safety standpoint, decomposition of a transition metal hydride (probably a TiFe alloy) is the best option for generating the needed hydrogen. However, the mass of substrate required to support $1 \mathrm{lb}$ of hydrogen may be too great for the exoskeleton application.

Of the individual hydrogen mechanisms considered, no single reaction appears to meet the need for hydrogen generation at a rate of $0.125 \mathrm{lb} / \mathrm{h}$ ( $1 \mathrm{lb}$ over an 8 -h period) without presenting significant engineering challenges. The hydrolysis reactions would require design of a compact, low-weight, heat-transfer system to remove the heat produced by reaction and with the exception of the catalytic reaction of sodium borohydride, will result in the gradual build-up of a corrosive hydroxide solution. This corrosive hydroxide solution will eventually have to be disposed of or neutralized.

In addition to the single-step processes evaluated, a two-step operation has been considered in which calcium hydride is hydrolyzed and the hydroxide is then neutralized with nitric acid. The combined heat generated per gram mole of hydrogen generated is $32.96 \mathrm{kcal}$. The ratio of reactant-to-hydrogen mass is 66.3. Based on the heat released and the mass ratio, this approach is less favorable than decomposition of a transition metal hydride.

\section{ICEs}

In general, ICEs are more efficient when the overall energy output is high in comparison to the power output - in other words, the weight of the fuel is high in comparison to the weight of the engine. Although this is not the case for the exoskeleton application, ICEs are still an option worthy of consideration. Reciprocal diesel engines and turbines, in particular, will be discussed. While new developments are currently being pursued for both small diesel and turbines, which will increase their attractiveness for an exoskeleton application, noise, heat, and emissions remain issues that must be confronted, generally at the expense of increased weight. Gasoline engines, which are more readily available in the 1 to $2 \mathrm{~kW}$ range, were not considered a viable alternative for a fieldable system due to the hazards associated with gasoline.

The power density of 1 to $40 \mathrm{~kW}$ diesel engines typically range from 100 to $400 \mathrm{~W} / \mathrm{kg}$, with fuel consumption in the 200 to $300 \mathrm{~g} / \mathrm{kWh}$ range (Theis, 2000; Heywood, 1988). Fuel consumption is generally increased in smaller scale diesel engines and generators. There are few diesel engines available in the lower power ranges. While a company called D-Star Engineering Corporation is currently making smaller scale diesels and is planning a $1 \mathrm{~kW}$ engine and generator set, it is doubtful that these will be available within the next one to two years.

In general, turbine fuel consumption would be anticipated to be from 5 to $15 \%$ less efficient than that for reciprocating diesels (Andriulli, 1999; Heywood, 1988). Micro-turbine performance does not scale well below $25 \mathrm{~kW}$ and fuel consumption tends to increase more in comparison to piston engines at partial loads (Andriulli, 1999). However, there are micro and mesoscopic turbine generators currently being developed under DARPA sponsorship, which offer the possibilities of compact power sources, which could be advantageous. These turbine generators, 
if successful, could potentially have specific powers up to $1,000 \mathrm{~W} / \mathrm{kg}$ and specific energies to $3,500 \mathrm{Wh} / \mathrm{kg}$.

\section{Batteries}

A variety of battery options are currently commercially available. Table 15 and Fig. 103 summarize the specific power and specific energy for a number of available batteries.

The difficulty of meeting both the power and energy requirements while minimizing the weight is a concern with batteries. However, some recent developments could make the use of batteries more desirable for the exoskeleton application. First, AeroVironment, Inc., has recently tested $\mathrm{NiMH}$ batteries (both Energizer ${ }^{\mathrm{TM}}$ and Panasonic ${ }^{\mathrm{TM}}$ ) that have power densities of $1 \mathrm{~kW} / \mathrm{kg}$ for up to $10 \mathrm{~s}$ with a resulting drop in storage efficiency to only $2.8 \mathrm{Wh} / \mathrm{kg}$. Such a battery could be very useful for meeting peak loads in a hybrid system. Also, another company EVonyx has developed a zinc air power cell (sometimes referred to as a semi-fuel cell) that shows significant promise. The power cell utilizes a replaceable zinc fuel card that can be disposable or rechargeable. If the projected improvements in specific energy and specific power are met, the cell could meet both energy and power requirements at a reasonable weight and could be a viable potential power source for the exoskeleton application.

Table 15. Battery comparison

\begin{tabular}{|c|c|c|c|}
\hline Type & Specific Energy $(\mathrm{Wh} / \mathrm{kg})$ & Specific Power $(\mathrm{W} / \mathrm{kg})$ & Cycles to $80 \%$ \\
\hline \hline Pb-acid & $30-45$ & 200 & $200-1000$ \\
\hline $\mathrm{Ni}-\mathrm{Cd}$ & $40-55$ & $190-260$ & $500-1000$ \\
\hline $\mathrm{NiMH}$ & $50-70$ & $180-200$ & $500-1000$ \\
\hline $\mathrm{Li}$ ion & $100-250$ & $300-800$ & $500-1200$ \\
\hline $\mathrm{Ag}-\mathrm{Zn}$ & $140-200$ & $100-330$ & $100-250$ \\
\hline $\mathrm{Ag}-\mathrm{Cd}$ & $55-95$ & $100-220$ & $300-500$ \\
\hline Zn-Air & $150-300$ & $50-100$ & \\
\hline Al-Air & 350 & $500-600$ & \\
\hline
\end{tabular}

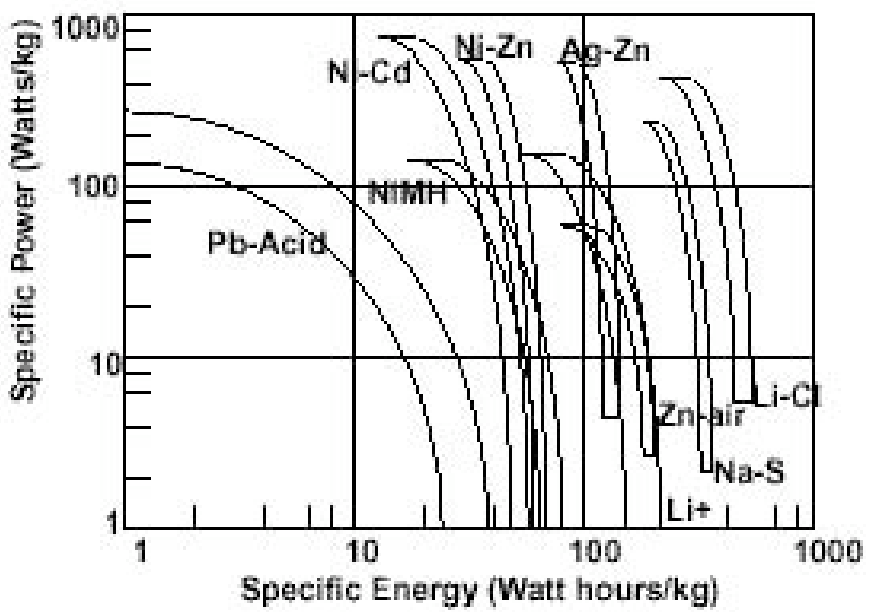

Fig. 103. Battery Ragone Diagram.

(Source: Dowling, January 1997.) 


\section{Super Capacitors}

Super capacitors could also be used in a hybrid system to meet peak power demands. Figures 104 and 105 show the energy stored as well as the peak power available in super capacitors (data taken from Maxwell Technologies' Ultra Capacitor Series). From the figures, it can be seen that a relatively high specific power of $7 \mathrm{~kW} / \mathrm{kg}$ and a relatively low specific energy of $3.5 \mathrm{Wh} / \mathrm{kg}$ are available. Thus, the super capacitors can provide a significant amount of power over a short period of time and could be extremely useful for load leveling in hybrid systems.
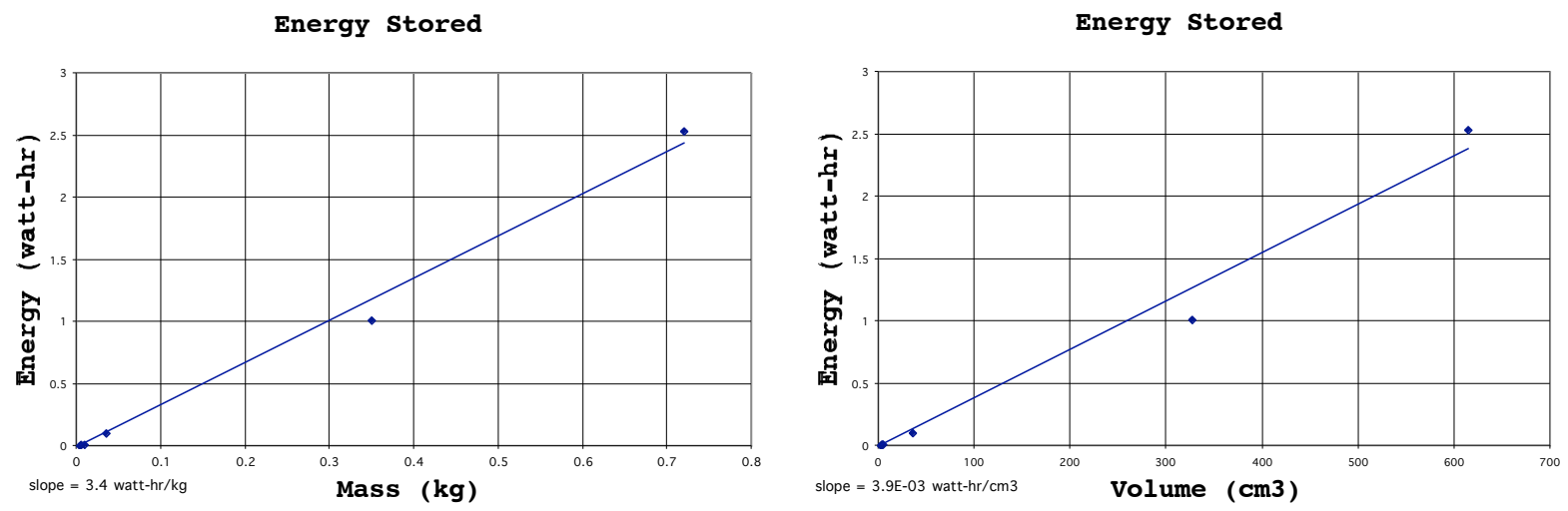

Fig. 104. Super capacitor energy storage vs mass and volume.
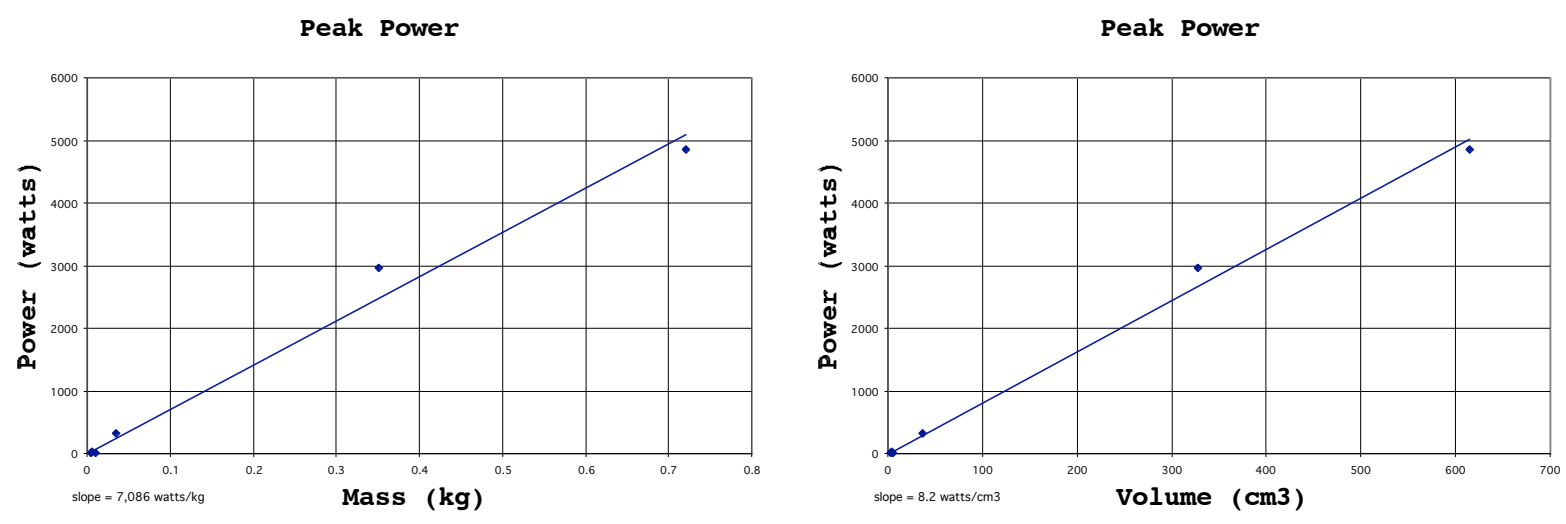

Fig. 105. Super capacitor peak power vs mass and volume.

\section{POWER SOURCES SUMMARY}

A power source for an exoskeleton must satisfy both power and energy requirements. With the significant variability in anticipated power demands, the ability of the power source to respond to peak loads without impeding performance is essential. Over-sizing a single power source to meet both power and energy requirements is not consistent with the need for efficiency required by this application. A hybrid power system, where transient peak power demands are met with one source and mission energy requirements are met with another source, can enhance efficiency at the expense of a more complicated system. Super capacitors and NiMH batteries are both capable of meeting peak transient load requirements, but they are insufficient as an energy 
source. A fuel cell fed by hydrogen, which is generated by a chemical reaction and an ICE both are capable of meeting system energy requirements.

Table 16 compares the weight for three possible power sources, diesel engine, fuel cell, and batteries, as well as four possible hybrid systems. While the weights for the ICE are lower than those for the fuel cell, it must be noted that the ICE weights do not include provisions for signature (noise, thermal, and emissions) reduction and vibration damping. These can be compensated for, but at the expense of increased complexity and weight.

Fuel cells allow for more stealthy operation because of their quiet operation, relatively low temperatures, and benign emissions (water). While there are development issues remaining to be solved for fuel cells, primarily regarding the hydrogen source, there appear to be viable options. These are currently being pursued by commercial suppliers and are likely to be readily available in the near future. Integrating a super capacitor or $\mathrm{NiMH}$ battery with a fuel cell fed by chemically generated hydrogen appears to be the most promising system, which can best meet the combination of power, energy, and signature objectives and safely function in close proximity to a human operator.

Table 16. Power source comparison

\begin{tabular}{|c|c|c|c|c|c|c|}
\hline Power Source Type & $\begin{array}{c}\text { Specific } \\
\text { Power } \\
(\mathrm{kW} / \mathrm{kg})\end{array}$ & $\begin{array}{c}\text { Specific } \\
\text { Energy } \\
(\mathrm{kWh} / \mathrm{kg})\end{array}$ & $\begin{array}{l}\text { Energy/ } \\
\mathrm{kg} \text { of Fuel } \\
(\mathrm{kWh} / \mathrm{kg})\end{array}$ & $\begin{array}{l}\text { Weight for } \\
\text { Power } \\
(\mathrm{kg})\end{array}$ & $\begin{array}{c}\text { Weight for } \\
\text { Energy }^{1}\end{array}$ & $\begin{array}{c}\text { Total } \\
\text { Weight } \\
(\mathrm{kg})\end{array}$ \\
\hline Diesel ICE ${ }^{2}$ & 0.4 & & 3.3 & 5.0 & 1.4 & 6.4 \\
\hline PEM Fuel Cell ${ }^{2,3}$ & 0.225 & & 1.4 & 8.9 & 3.4 & 12.3 \\
\hline Li Ion Batteries $^{4}$ & 0.5 & 0.25 & & 4.0 & 19.2 & 19.2 \\
\hline $\begin{array}{l}\text { Hybrid } \\
\text { Fuel Cell } \\
\text { Battery } \\
\text { Total }\end{array}$ & $\begin{array}{c}0.225 \\
1.0\end{array}$ & & 1.4 & $\begin{array}{l}2.7 \\
2.0\end{array}$ & 3.4 & 8.1 \\
\hline $\begin{array}{l}\text { Hybrid } \\
\text { Fuel Cell } \\
\text { Super Capacitor } \\
\text { Total } \\
\text { Tol }\end{array}$ & $\begin{array}{c}0.225 \\
7.0\end{array}$ & & 1.4 & $\begin{array}{l}2.7 \\
0.3\end{array}$ & 3.4 & 6.4 \\
\hline $\begin{array}{l}\text { Hybrid } \\
\text { Diesel ICE } \\
\text { Battery }^{6} \\
\text { Total } \\
\end{array}$ & $\begin{array}{l}0.4 \\
1.0\end{array}$ & & 3.3 & $\begin{array}{l}1.5 \\
2.0\end{array}$ & 1.4 & 4.9 \\
\hline $\begin{array}{l}\text { Hybrid } \\
\text { Diesel ICE } \\
\text { Super Capacitor } \\
\text { Total }\end{array}$ & $\begin{array}{l}0.4 \\
7.0\end{array}$ & & 3.3 & $\begin{array}{l}1.5 \\
0.3\end{array}$ & 1.4 & 3.2 \\
\hline
\end{tabular}

Requirements:

Avg Power $(\mathrm{kW})=\quad 0.6$

Peak Power $(\mathrm{kW})=2.0$

Energy $(\mathrm{kWh})=\quad 4.8$

1 Based on specific energy or fuel consumption.

Sized to meet peak power and energy requirements, including fuel.

Specific energy based on $40.0 \mathrm{kWh} / \mathrm{kg}$ (higher heating value) of $\mathrm{H}_{2}$. Assumes $7 \% \mathrm{H}_{2}$ by wt \& $50 \%$ efficiency.

Total weight based on energy requirements.

Sized to meet average power and energy requirements.

Sized to meet peak power requirements. 


\section{APPENDIX F \\ INTEGRATED POWER SYSTEM DEVELOPMENT}


AeroVironment, Inc. has documented the development of an integrated power system in a final report (AeroVironment, Inc., 2003) for the ORNL Subcontract 4000007000. This appendix provides the details regarding the design and development and is included here for completeness and the convenience of the reader.

\section{TECHNICAL APPROACH}

\section{Objective}

Our objective was construction of a demonstration power supply for our chosen hybrid architecture. We developed a five-step work plan to do so.

The first step was to compare and contrast off-the-shelf components in order to select the best possible components and system configuration. Second, we proceeded to design and build the power supply. We procured off-the-shelf components and combined them into subassemblies. The two main subassemblies were the battery pack and the fuel cell plus ancillaries. As a third step we tested, debugged, and integrated the power supply, and then carried out a series of tests performed in a manual mode where some portions of the control were done by hand.

Once convinced that the key subsystems work properly, the next step (Step 4) was to develop the system controller, and the additional hardware to measure and control all system parameters. In Step 5 we also added the chemical hydrogen generation subsystem from ORNL, prepared the sodium borohydride solution and started operating the system.

The result of the five-step approach was a prototype system ready to operate from fuel to electricity, capable of demonstrating hybrid system capabilities for the soldier's energy and power requirements.

The project finished with a preliminary CAD design of the integrated system. This preliminary design included a packaging design and analysis to support material selection and geometric layout of the system. We then fabricated the integrated system and tested it under several upper and lower bound power profiles.

\section{Approach Details}

The following details our technical approach.

\section{- Step 1: Performance models}

Component level: Simple spreadsheet level models for each component were constructed and evaluated in a system model. These models were mainly needed to evaluate the control algorithms.

System level: This model included steady state evaluations and transient effects and allowed for trade-off between different components and control strategies. Important conditions to model included base-load power at steady state, the intermediate power interval, the recharge transient, the peak pulse event, the startup event, and the shutdown event. Outputs included power and heat flows, required voltages and currents, energy levels, temperatures, mass flows, and voltages as a function of time. 
Control algorithms: The System level model was exercised to evaluate component options and control strategies. The results were performance plots used to help down-select components, system configuration, and control algorithms.

- Step 2: Hybrid Battery Pack - Fuel Cell System Design and Assembly

Board design selection: The analysis described above provided the necessary information and data to choose the system configuration and components.

Drawings of brass board parts and manufacture: The brass board was laid out in a 2D fashion on a single test bench. Plumbing, wiring, and position of components were designed in a 2D-drafting package.

Energy management system: Using a commercially available programmable controller we programmed the control algorithms determined in Step 1.

Battery pack assembly: The selected unit cells were integrated into a pack that fit with the board layout.

Battery pack testing: The test load profiles were determined based on the system modeling done in Step 1. Next, the pack was tested using our in-house battery test equipment and temperature effects were modeled.

Fuel cell assembly and related ancillaries: As with the battery, test load profiles were determined based on the system modeling done in Step 1. Then, the fuel cell was tested using in-house battery test equipment and temperature effects were modeled.

Fuel cell testing: The fuel cell was tested over all of the operational conditions.

Hybrid system assembly: Once the subassemblies had been tested and debugged, the entire system was assembled and the system test plan was refined.

- $\quad$ Step 3: Testing

Evaluation of component and system options: First, the assembled system was tested under manual control using simple load resistors. Next, full steady state polarization, peak pulse and recharge events were measured using a PC based A/D converter and fast FET power switch. Startup and shutdown sequences were tested manually.

Mechanical and analog electrical issues: Hardware modifications were made in response to mechanical, electrical, or data acquisition system exercises.

Control algorithm adjustments: Control logic was refined based on manual testing.

- Step 4: Digital Control

Testing - digital control: The full board was tested under digital control using baseload power, intermediate power, peak pulse, shutdown and startup sequences.

Digital control issues: Remaining controls, hardware, or electrical issues were addressed.

- Step 5: Hydrogen System Integration

Acquisition and Assembly: The hydrogen generation catalytic reactor was obtained from ORNL. A layout was made for subassemblies necessary to integrate this reactor into the prototype system.

Fuel Mix: An investigation was made of the mixture start points for the sodium hydroborohydride solution.

Integration: The system, including its controllers was assembled into the prototype.

Testing: The full prototype was tested under digital control using baseload power, intermediate power, peak pulse, shutdown and startup sequences. 


\section{RESULTS}

\section{Battery Component, Model and Results}

\section{Selection}

NiMH batteries were chosen because of the combination of high specific energy and high power density for this type of battery. The same battery technology is being used in several models of hybrid electric vehicles. In a hybrid application, these batteries can perform hundreds of thousands of load-leveling transient cycles over years of vehicle operation, so there is little doubt that it will be sufficient for the exoskeleton power supply.

Batteries can be ranked by their ability to deliver peak specific power. For NiMH batteries, most of the resistance is taken to be Ohmic, so that ranking by peak power also ranks the batteries in order of power deliverable at a given efficiency. The peak power rating is given by

$$
\frac{\mathrm{P}_{\max }}{\mathrm{m}} \sim \frac{\mathrm{V}_{0}}{2}=\frac{1}{\mathrm{Rm}}
$$

where $\mathrm{m}$ is the cell mass, $\mathrm{V}_{0}$ is the effective open circuit voltage, $\mathrm{R}$ is the effective cell resistance, and $\mathrm{V}$ is the voltage. The fraction of peak power can be computed from the discharge/charge voltages via

$$
\left.\frac{\mathrm{P}}{\mathrm{P}_{\max }}=4 \frac{\mathrm{V}}{\mathrm{V}_{0}} \mathrm{~L}\right] \frac{\mathrm{V}}{\mathrm{V}_{0}}[-
$$

The $\mathrm{V}_{0}$ and $\mathrm{R}$ are functions of the given state of charge, temperature, and sign of current (i), and satisfies the $V$, current relationship for the battery,

$$
\mathrm{V}=\mathrm{V}_{0} \square \mathrm{R} \text { i }
$$

in the state of charge range from 60 to $80 \%$.

Table 17 ranks some commercially available NiMH batteries at $20^{\circ} \mathrm{C}$ and $80 \%$ state of charge. The resistance of a NiMH battery varies with temperature and is mainly a function of temperature, and to a lesser degree a function of state of charge and current draw. Since the batteries will operate in a higher temperature environment, we can extrapolate the performance by noting that there is probably a $30 \%$ increase in power at $40^{\circ} \mathrm{C}$ vs at $20^{\circ} \mathrm{C}$. In hybrid applications, these batteries are typically not run below $0.95 \mathrm{~V}$ (or $75 \%$ of peak power), or above $1.4 \mathrm{~V}$ for efficiency and thermal reasons. Miscellaneous losses associated with interconnections, and battery variations are on the order of $20 \%$. Packaging adds $10 \%$ to the weight and decreases the specific power by $10 \%$. Taking all of these factors into consideration, the rated power (i.e., he power we are willing to use) at $40^{\circ} \mathrm{C}$ is approximately $74 \%$ of the peak power at $20^{\circ} \mathrm{C}$.

As can be seen from the Table 17, each company's smallest cells have the highest power to weight. 
Table 17. Peak specific power of various commercial NiMH Cells

(At $20^{\circ} \mathrm{C}$ and $80 \%$ state of charge, based on data from manufacturers' literature.)

\begin{tabular}{|c|c|c|c|c|c|c|}
\hline Vendor & Model & $\begin{array}{c}\text { Effective } \\
\text { Open Circuit } \\
\text { Voltage, } \mathrm{V}_{0} \\
(\mathrm{~V})\end{array}$ & $\begin{array}{c}\text { Effective } \\
\text { Internal } \\
\text { Resistance, } \mathrm{R} \\
(\mathrm{mOhm})\end{array}$ & $\begin{array}{c}\text { Bare Cell } \\
\text { Mass, } \mathrm{m} \\
(\mathrm{g})\end{array}$ & $\begin{array}{c}\text { Peak Specific } \\
\text { Power @ 20C, } \\
\mathrm{P} / \mathrm{m}(\mathrm{W} / \mathrm{kg})\end{array}$ & $\begin{array}{c}\text { Design Specific } \\
\text { Power @ 40 } \mathrm{C}, \mathrm{P} / \mathrm{m} \\
(\mathrm{W} / \mathrm{kg})\end{array}$ \\
\hline Panasonic & HHR200SCP & 1.27 & $10.0^{*}$ & 42 & 914 & 677 \\
\hline Panasonic & HHR300SCP & 1.27 & $8.5^{*}$ & 55 & 863 & 638 \\
\hline Panasonic & HHR650D & 1.27 & $3.5^{*}$ & 170 & 678 & 501 \\
\hline Moltech & EMH-1550C & 1.27 & 14.0 & 40 & 720 & 533 \\
\hline Moltech & EMH-2200C & 1.27 & 11.5 & 53 & 662 & 490 \\
\hline Moltech & EMH-2500C & 1.27 & 11.0 & 58 & 632 & 468 \\
\hline Moltech & EMX-7000D & 1.27 & 7.0 & 160 & 360 & 266 \\
\hline
\end{tabular}

${ }^{\star}$ Estimated from discharge curves on data sheets.

The highest power cells from Panasonic (HHR200SCP and HHR300SCP), and Moltech (EMH1550C and EMH2200C) were purchased for testing.

The testing range chosen for charge and discharge power at states of charge was 20 to $100 \%$ and temperatures were 20 to $45^{\circ} \mathrm{C}$. This test matrix provided sufficient information for creation of a look-up table model to be used for simulation.

\section{Modeling}

The component model for the battery included state variables of current, i, state of charge, $\square$, and temperature, T. All other quantities are derived from these. The voltage for a NiMH battery was estimated using the equation

$$
\mathrm{V}=\mathrm{V}_{0}(\square, \mathrm{T}, \mathrm{i}) \square \mathrm{R}(\square, \mathrm{T}, \mathrm{i}) \mathrm{i}
$$

to relate the voltage to the state variables. The effective open circuit voltage, $\mathrm{V}_{0}$, and effective cell resistance $\mathrm{R}$ are functions of the state of charge and were, for our purposes, computed via look-up tables derived from experimental data. For battery operation around a specific temperature and state of charge, the equations were also linearized.

a) State of charge calculation: The state of charge was computed by amp-hour integration of the battery current. It is assumed that there were no coulombic losses in the state-of-charge range of interest so that,

$$
\frac{\mathrm{d} \square}{\mathrm{dt}}=\square \frac{1}{3600 \mathrm{~s}} \frac{\mathrm{i}}{\mathrm{i}_{\mathrm{c}}}
$$

where $i$ is the current through the battery that is positive on discharge, and negative on charge, and $i_{c}$ is the $1 \mathrm{C}$ (or 1 hour) discharge current. The factor of $3600 \mathrm{~s}$ assumed that the units of time are seconds.

b) Cell temperature calculation: The battery heat generation assumed only $i^{2} R$ losses, and was estimated as a multiple of the output power using, for example, 


$$
\frac{\mathrm{q}_{\text {ohmic }}}{\mathrm{P}_{\text {out }}}=\frac{\mathrm{i}^{2} \mathrm{R}}{\mathrm{Vi}}=\frac{\mathrm{iR}}{\mathrm{V}}=\frac{\mathrm{V}_{0}}{\mathrm{~V}} \square 1
$$

The battery heat was assumed to be generated uniformly within the volume of the battery, and lost from the surface. The temperature, $\mathrm{T}_{\text {cell }}$, is the state for the thermal model of the battery, simply put, the rate of temperature change is proportional to the net increase in heat so that,

$$
\frac{\mathrm{dT}_{\text {cell }}}{\mathrm{dt}}=\frac{1}{\mathrm{mc}_{\mathrm{p} \text {,battery }}}\left\{\mathrm{i}^{2} \mathrm{R} \square \mathrm{hA}_{\text {cell }}\left(\mathrm{T}_{\text {cell }} \square \mathrm{T}_{\text {air }}\right)\right\},
$$

where $\mathrm{T}_{\text {cell }}$ is assumed uniform over the cell, $\mathrm{T}_{\text {air }}$ is the average of the inlet and outlet air temperature, and $c_{p, b a t t r y}$ is the heat capacity of the battery. The first term in the brackets is the ohmic heating of the cell, the second is the heat removed by forced convection. The heat capacity of the battery was estimated to be close to that of water, on a volume basis (i.e., $4.2 \mathrm{~kJ} / \mathrm{LK}$ ). A NiMH battery is approximately $\sim 3.3 \mathrm{~kg} / \mathrm{L}$, so that the heat capacity was estimated to be $1.3 \mathrm{~kJ} / \mathrm{kgK}$.

c) Convection cooling calculation: For the convective heat transfer (cooling), we assumed that the cells were arranged side-by-side, end-on into the flow so that the exposed surface area of a cylindrical cell included the ends and thus,

$$
\mathrm{A}_{\text {cell }}=\square \mathrm{DL}+\frac{\square}{2} \mathrm{D}^{2}=\square \mathrm{D}^{2} \frac{\square \mathrm{L}}{\square \mathrm{D}}+\frac{1}{2}=\text {. }
$$

If fins are used, then this area is increased by the fin area. For small temperature differences, the heat transfer coefficient was estimated using,

$$
\mathrm{h} \sim 1.86 \overbrace{\mathrm{L} / \mathrm{D}_{\mathrm{h}}}^{\mathrm{RePr}} \mathrm{\square}^{/ 3}
$$

The hydraulic diameter, $\mathrm{D}_{\mathrm{h}}$, is defined as four times the ratio of the open area to the wetted perimeter. For the current geometry that does not use fins, this is

$$
\mathrm{D}_{\mathrm{h}}=\mathrm{D}\left(\frac{4}{\square} \square 1\right) \sim 0.27 \mathrm{D}
$$

The Reynolds number is,

$$
\operatorname{Re}=\frac{U_{h}}{\square}
$$

where $U$ is the average flow speed in the channels, and $\square$ is the kinematic viscosity.

To keep a high heat exchanger effectiveness, we wanted the (average) flow rate to be sufficient to carry the heat from the cell,

$$
\square \mathrm{A}_{\mathrm{cs}} \mathrm{Uc}_{\mathrm{p}, \text { air }} \square \mathrm{T}_{\text {air,out } \square \mathrm{in}} \sim \mathrm{q}_{\mathrm{ohmic}},
$$


where $A_{c s}$ is the cross-sectional or open flow area, and $c_{p, a i r}=1 \mathrm{KJ} / \mathrm{kgK}$ is the heat capacity for air. The pressure rise through the battery pack is dominated by abrupt expansion losses and is on the order of,

$$
\square \mathrm{p}_{\text {cool }}=0.25 \square \overline{\mathrm{U}}^{2}
$$

and the minimum cooling power (not including blower efficiencies) is then,

$$
\mathrm{P}_{\text {cool }}=0.25 \square \overline{\mathrm{U}}^{3} \mathrm{~A}_{\mathrm{cs}} .
$$

d) Calculation of temperature variation within the cell

We assumed a simple one dimensional scaling, the maximum temperature difference from the center of the battery to the walls given by the steady state temperature distribution for uniform heating and cooling,

$$
\square \mathrm{T}_{\text {max,cell }} \sim \frac{\mathrm{q}}{4 \square \mathrm{k} \mathrm{L}}
$$

where $\mathrm{q}$ is the net heat generation by the cell, $\mathrm{k}$ is the thermal conductivity of the cell, and $\mathrm{L}$ is the cell length.

For the 4/5 Sub-C cell, we estimated the order of magnitude conductivity to be greater than $1 \mathrm{~W} / \mathrm{mK}$, so that for the small cells, with $\mathrm{q} \sim 30$, and $\mathrm{L} \sim 3.3 \mathrm{~cm}$, the maximum temperature difference is

$$
\square \mathrm{T}_{\text {max }, \text { cell }}<\frac{30 \mathrm{~W}}{4 \square 10 \frac{\mathrm{W}}{\mathrm{mK}} 0.033 \mathrm{~m}} \sim 7 \mathrm{~K},
$$

We chose to neglect this for our purposes, as we expected the thermal resistance at the cell-air interface to be much larger than the internal thermal resistance, so that the steady state temperature distribution would not be reached, and the internal temperature would be close to uniform.

\section{Testing}

Four battery packs, one for each battery model, were tested to determine their performance and to obtain modeling data. Each battery pack consisted of 12 cells connected in series, shrinkwrapped, with leads and a connector. Twelve cells were used (as opposed to single cells) so that realistic interconnect resistance, and packaging weight would be taken into account.

The current draw, during ten-second high rate charge (at $1.45 \mathrm{~V}$ ) and high rate discharge (at $0.85 \mathrm{~V}$ ) pulses, was measured at state of charge (SOC) levels from $100 \%$ to $10 \%$. In-between SOC levels, the batteries were charged and discharged at a nominal "base load" current. The test data are summarized in Figs. 106 through 109 and Tables 19 through 21.

These tests were performed at room temperature $\left(22^{\circ} \mathrm{C}\right)$ and $45^{\circ} \mathrm{C}$ for all four of the candidate battery packs, and at $\left(50^{\circ} \mathrm{C}\right)$ for the Moltech EMH-2200C (Table 19) that is specified to operate at a somewhat higher temperature. 


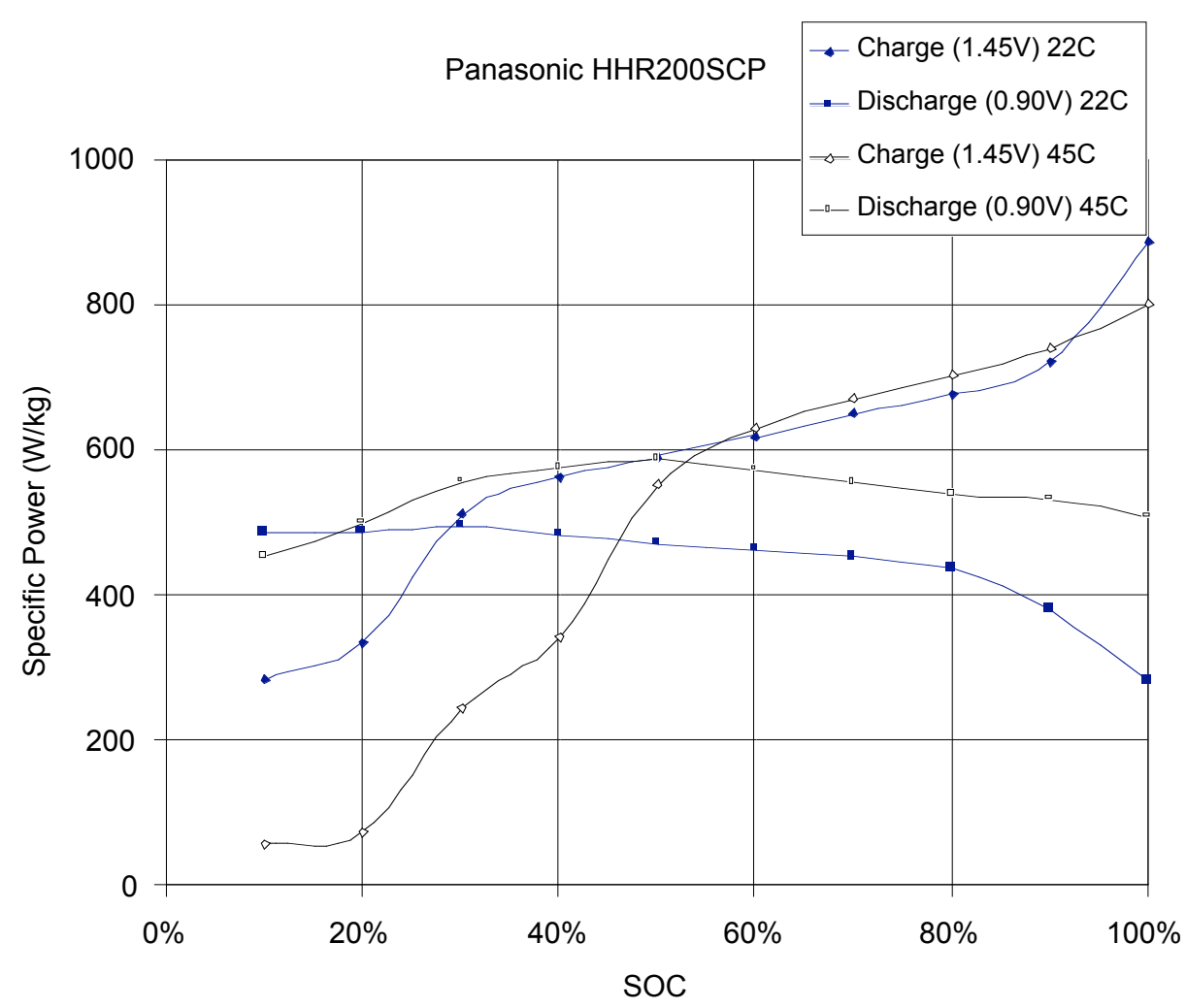

Fig. 106. Specific power curves for Panasonic HHR200SCP batteries.

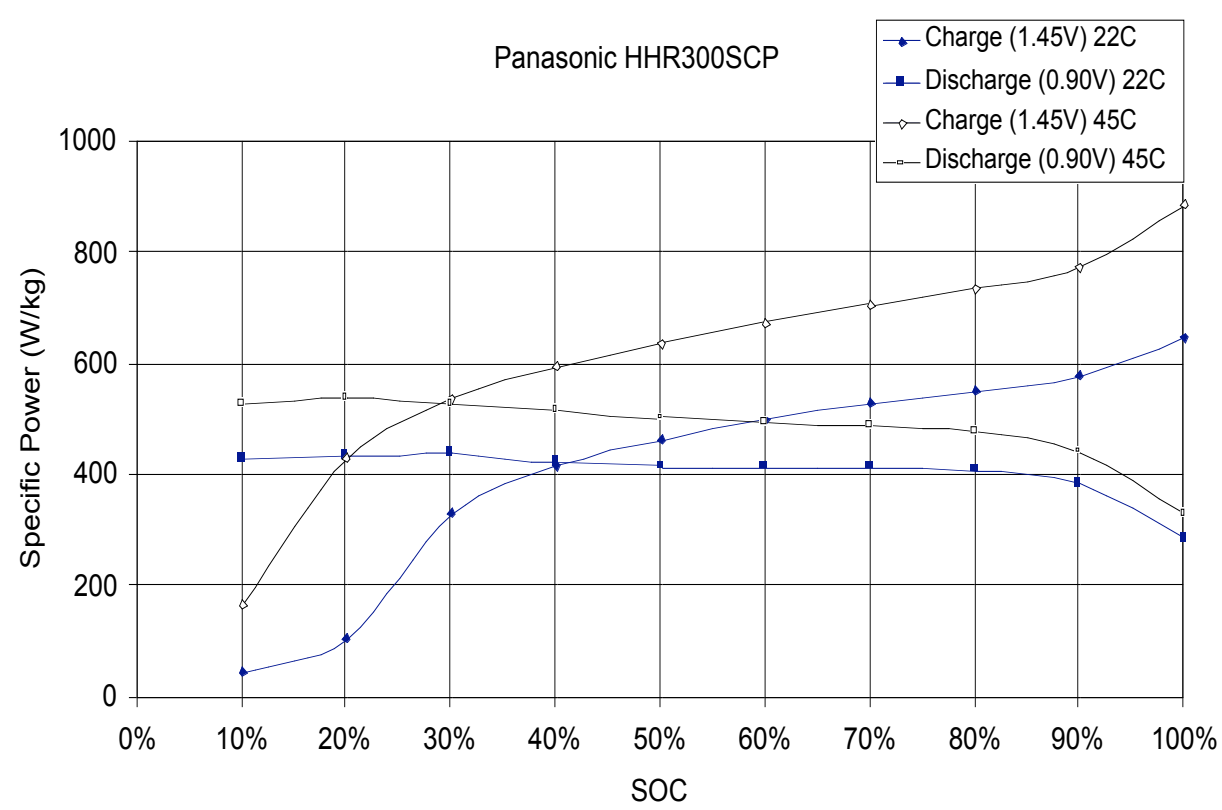

Fig. 107. Specific power curves for Panasonic HHR300SCP batteries. 


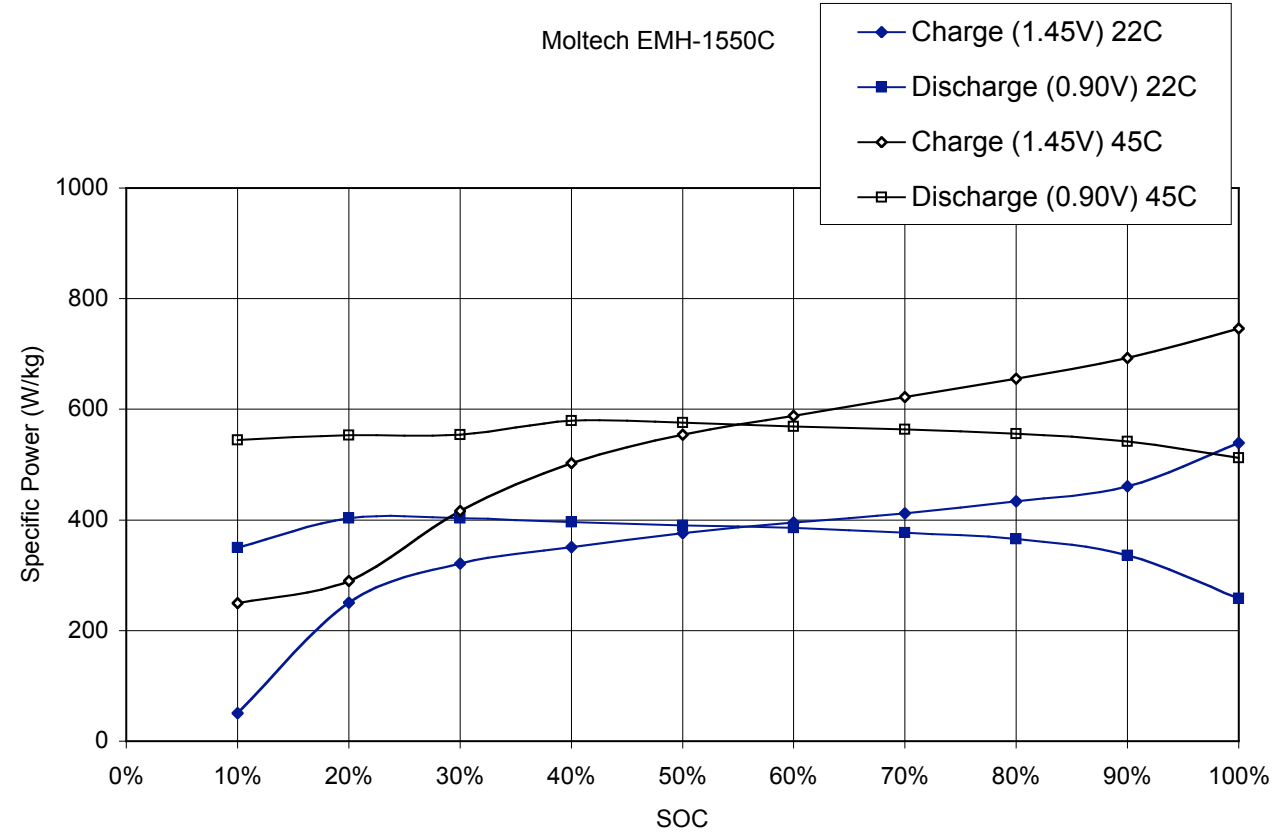

Fig. 108. Specific power curves for Moltech EMH-1550C batteries.

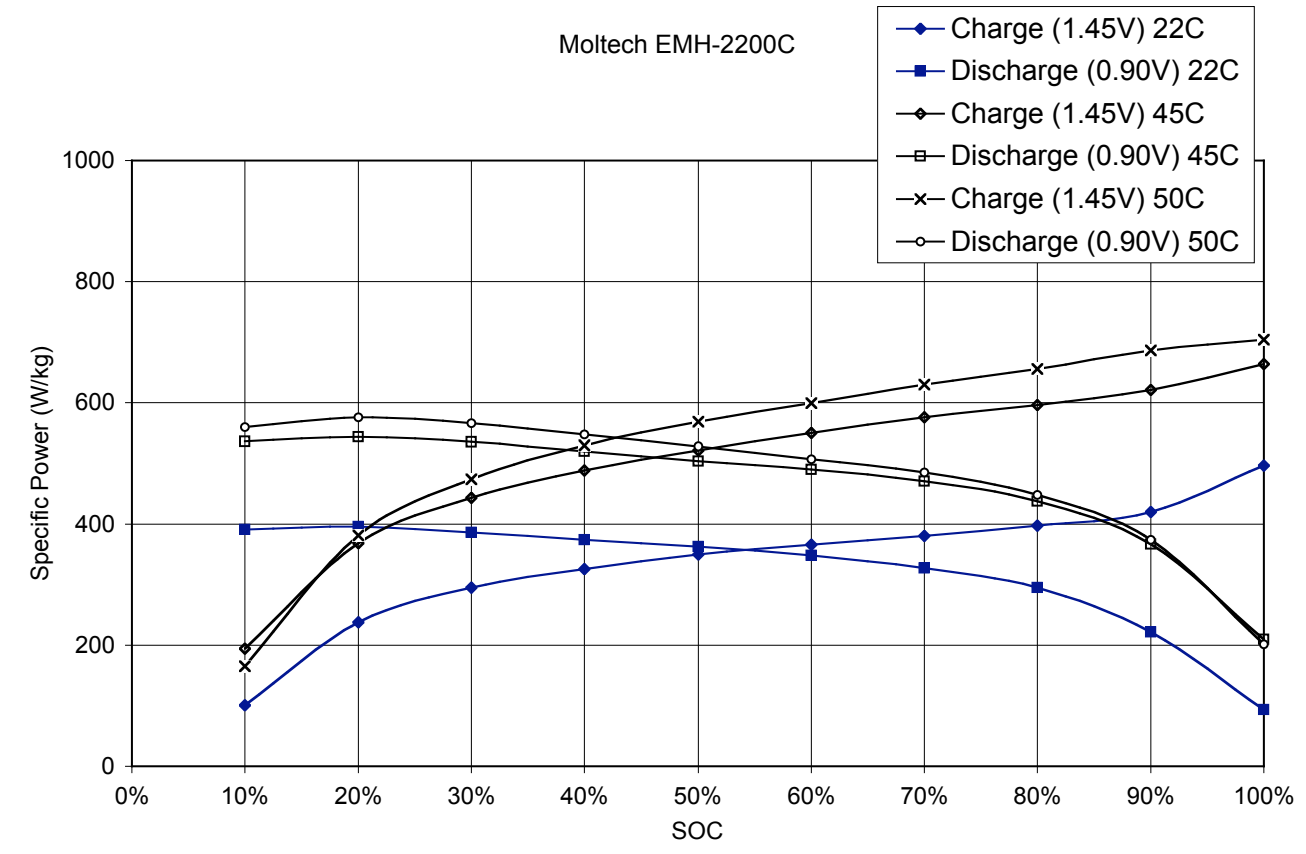

Fig. 109. Specific power curves for Moltech EMH-2200C batteries. 
Table 18. Pack voltage for $1.5 \mathrm{~kW}$ discharge capabilities

(Battery pack is $2.3 \mathrm{~kg}$, which assumes a $650 \mathrm{~W} / \mathrm{kg}$ specific power.)

\begin{tabular}{|c|c|c|c|}
\hline Battery & $\begin{array}{c}\text { Number of } \\
\text { Cells }\end{array}$ & $\begin{array}{c}\text { Pack Voltage } \\
\text { At 0.9 V/Cell }\end{array}$ & $\begin{array}{c}\text { Pack Voltage } \\
\text { At 1.45 V/Cell }\end{array}$ \\
\hline H-HR200SCP & 51 & 46 & 74 \\
\hline HHR300SCP & 39 & 35 & 57 \\
\hline
\end{tabular}

Table 19. Panasonic HHR200SCP data

(Data is for packs of 12 cells, but is presented on a per-cell basis.)

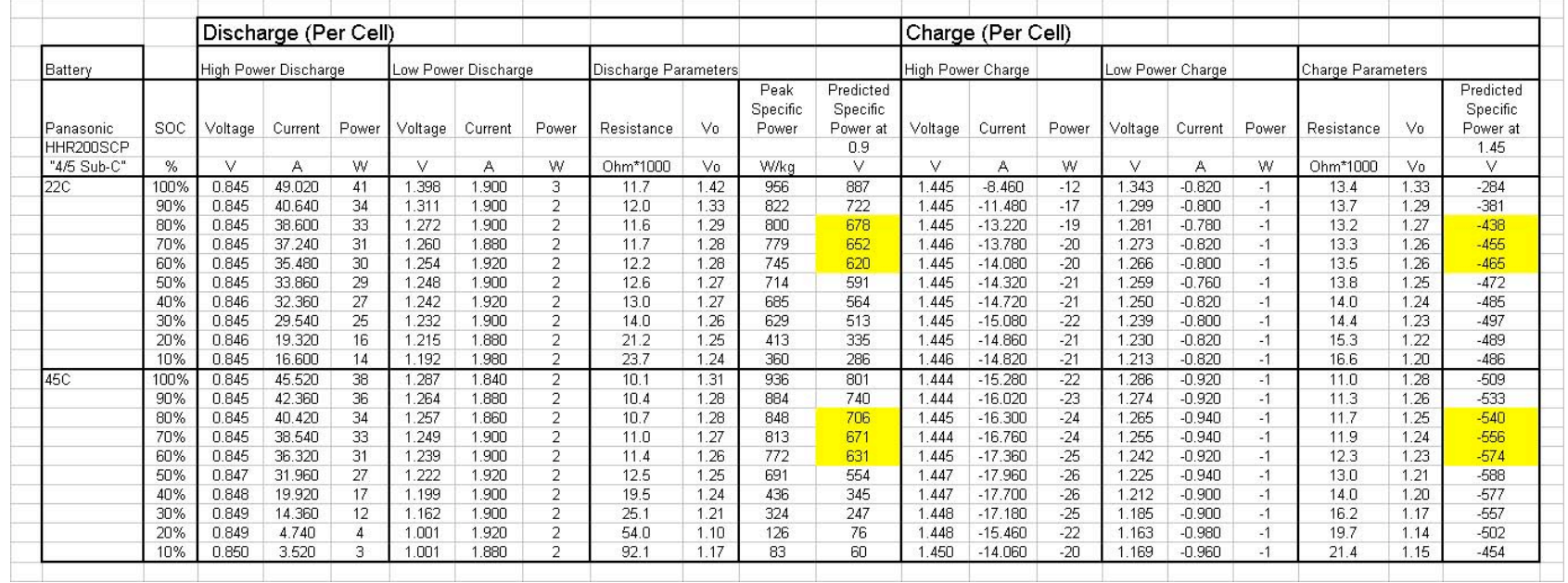

Table 20. Panasonic HHR300SCP data

(Data is for packs of 12 cells, but is presented on a per-cell basis)

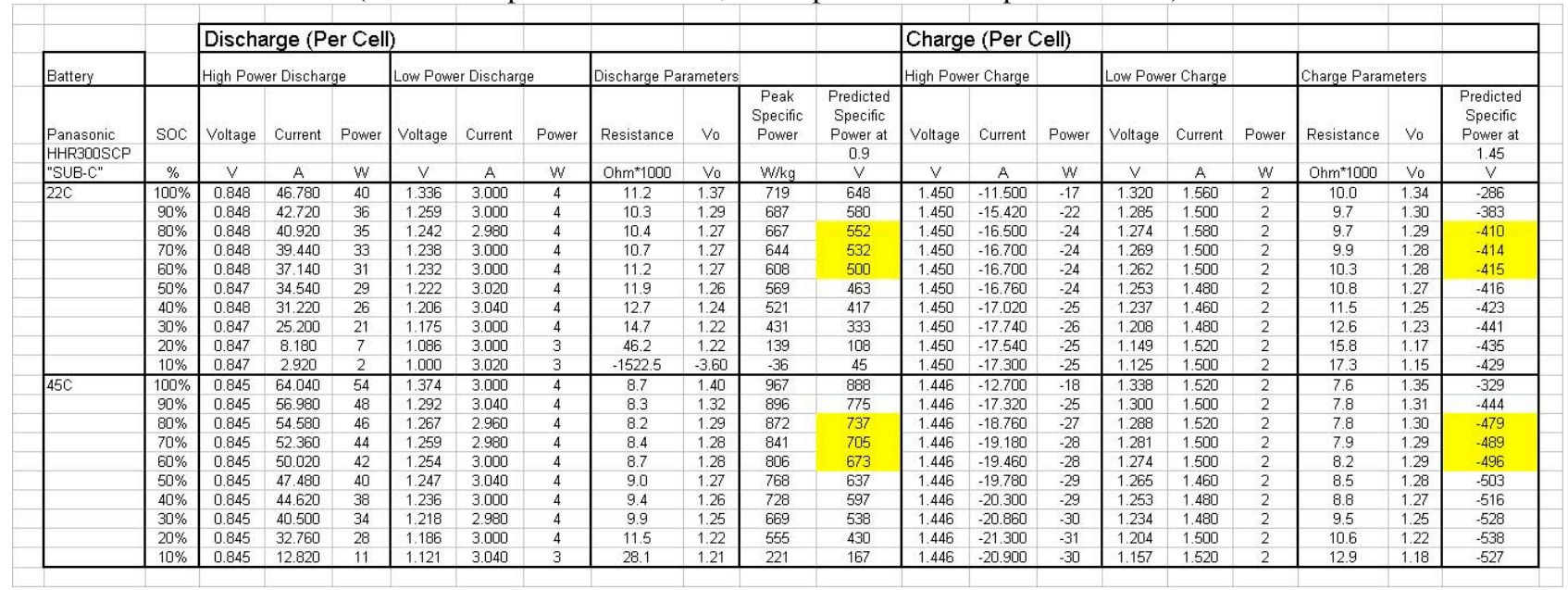


Table 21. Moltech EMH-1550C data

(Data is for packs of 12 cells, but is presented on a per-cell basis.)

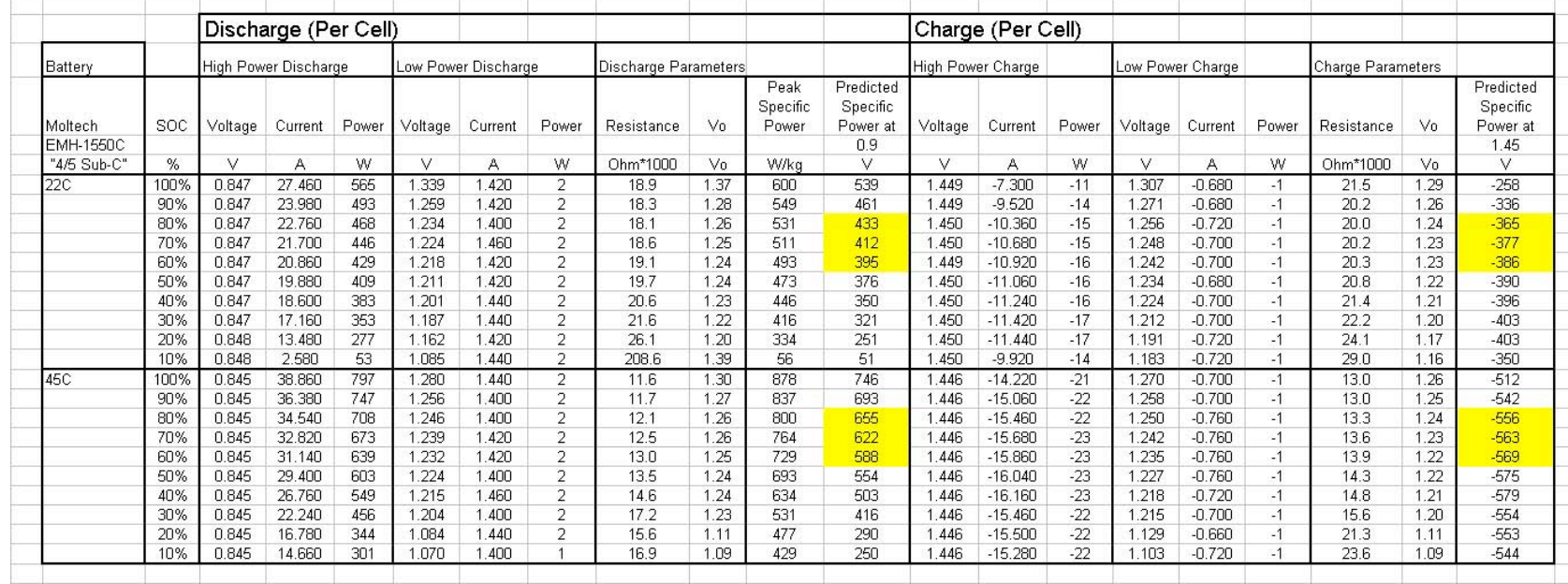

Table 22. Moltech EMH-2200C

(Data is for packs of 12 cells, but is presented on a per-cell basis.)

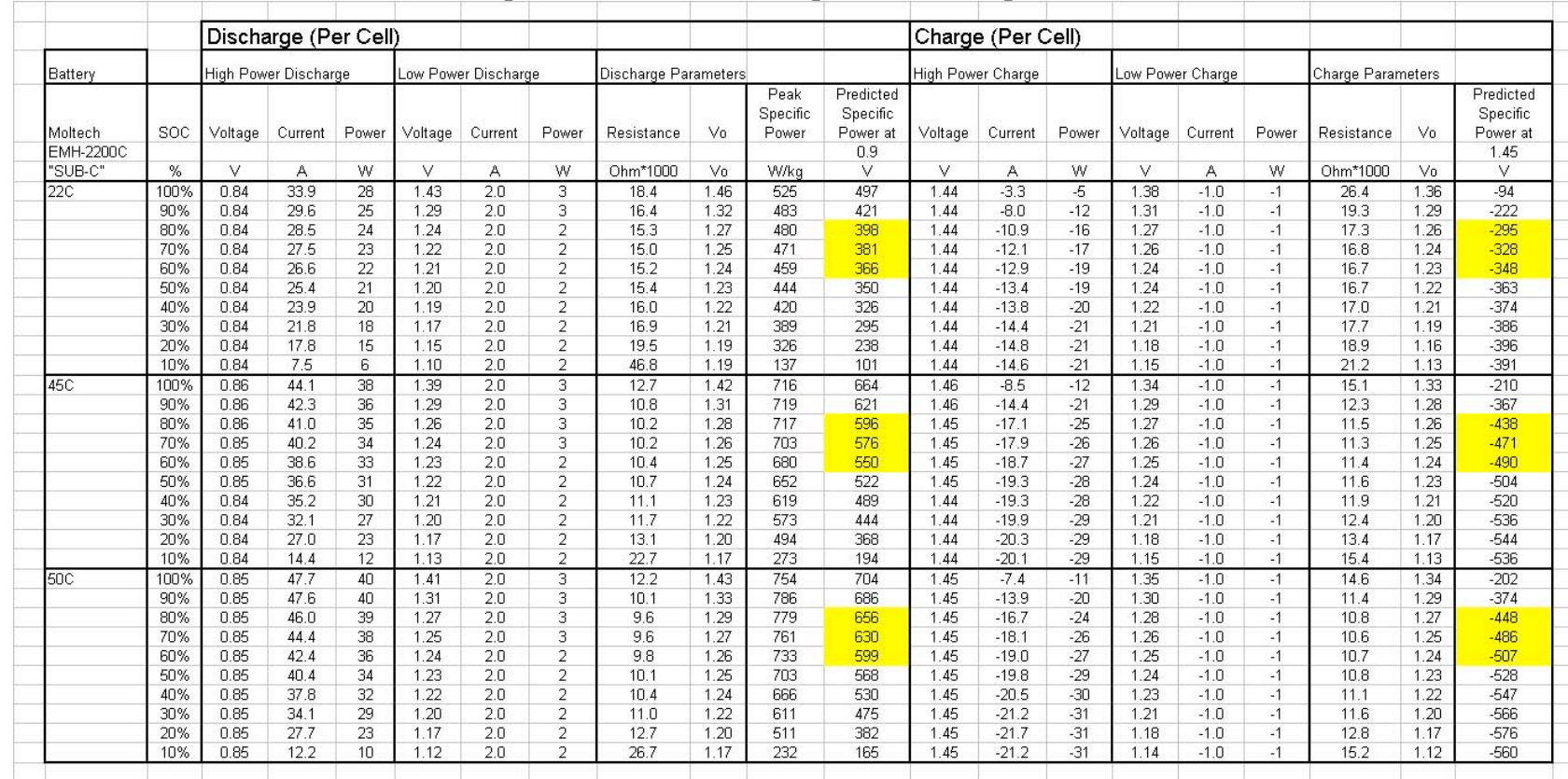

The effective resistance $(\mathrm{R})$ and offset voltage $\left(\mathrm{V}_{0}\right)$ were estimated from the data for charge and discharge at each SOC level, so that the simple equation,

$$
\mathrm{V}=\mathrm{V}_{0} \square \mathrm{R} \text { i, }
$$

yielded the correct V-i (i.e., Voltage-current) relationship at each SOC level. For simulation purposes, $\mathrm{V}_{0}$ and $\mathrm{R}$ were computed using a look-up table that is a function of SOC and temperature.

The estimated charge and discharge specific powers are also presented in the Tables 18 through 21 and Figs. 106 through 109. The peak discharge power was of academic interest, but showed that these batteries approached $1000 \mathrm{~W} / \mathrm{kg}$ power capability (for 10 second pulses). However, 
this level of power was only realized with a fully charged battery, and that was not an appropriate condition when these batteries are to be used for load leveling. The specific powers computed at $0.9 \mathrm{~V}$ (discharge) and $1.45 \mathrm{~V}$ (charge), and 60\%-80\% state of charge more closely represented the normal allowable range of operation. We noted that the specific power computed below was based on the battery pack weight including interconnects and packaging.

The shaded areas of the tables indicated the specific power for charge and discharge in the normal range of operation. As seen from the data, all of the batteries performed well, and better at the higher temperatures. On average, the Panasonic batteries performed somewhat better than the Moltech batteries, with the Panasonic HHR200SCP besting the Panasonic HHR300SCP in charge performance, and in discharge performance at room temperature. Interestingly, the HHR200SCP performed somewhat worse than the HHR300SCP at the higher discharge temperature, but not significantly. The performance at lower temperatures was not tested, but it was known that the performance decreases similarly at even lower temperatures. We noted that the batteries could be heated up relatively easily, so operation at higher temperatures was preferred.

Table 17 illustrated the pack voltage that was implied by a $1.5 \mathrm{~kW}$ power requirement assuming a $650 \mathrm{~W} / \mathrm{kg}$ battery pack. The obvious implication was that there needed to be a DC/DC between the fuel cell and battery pack. For a high efficiency lightweight, DC/DC conversion, we could expect $10 \mathrm{~W} / \mathrm{g}$, or 60 grams for a $600 \mathrm{~W}$ fuel cell.

Parameters to simulate the charge and discharge (electrical) characteristics of the present $\mathrm{NiMH}$ batteries were determined experimentally. Either of the Panasonic batteries were recommended for use in transient handling. We also found that almost $1 \mathrm{~kW} / \mathrm{kg}$ is realizable with these batteries, and this was the figure used for the original performance estimates. However, we found that 600-650 W/kg (on discharge) and 400-500 W/kg (on charge) were more realistic for the present transient handling application.

\section{Results: Fuel Cell Component}

\section{Selection}

A nominally $500 \mathrm{~W}$ Fuel cell system from H-Power (PowerPPM-PS500) was selected for testing. The purpose of the energy storage system is to provide a lightweight refuelable electric power source for a human exoskeleton or enhanced mobility device described above.

\section{Modeling}

The performance of a fuel cell operating with pure hydrogen as fuel and air as oxidant at a given constant temperature can be modeled using the following equation (Wakizoe, 1995),

$$
\mathrm{E}=\mathrm{E}_{0} \square \mathrm{b} \log _{10} \stackrel{\square}{\mathrm{i}_{\mathrm{i}}} \stackrel{\square}{\square} \square \mathrm{Ri}+\mathrm{b} \log _{10} \frac{\square \mathrm{p}}{-\mathrm{p}_{0}} \square,
$$

where $\mathrm{i}$ is the current density in $\mathrm{A} / \mathrm{cm}^{2}, \mathrm{i}_{\mathrm{o}}=0.001 \mathrm{~A} / \mathrm{cm}^{2}$ is the exchange current density, $\mathrm{E}_{0}$ is the open circuit voltage, $\mathrm{b}$ is the rate of decrease of voltage with current density in V/decade, and $\mathrm{R}$ 
is the cell internal resistance per unit area, $\square . \mathrm{cm}^{2}, p$ is the operating pressure, and $\mathrm{p}_{0}=14.7 \mathrm{psi}$ is the reference pressure.

This equation assumes that a sufficient amount of hydrogen and air are supplied to the fuel cell. When the cell gets starved its behavior becomes more complicated and cannot be described by such a simple equation.

For the purposes of our first approximation model, we wanted to be conservative so we required that the fuel cell operate within the assumptions of the equation above, i.e., power would not be drawn in excess of the available reactants. The flow rate of hydrogen and of the air supply were used to define the peak power possible at any moment, so time response of the fuel cell would be determined by the time response of the fuel and air supplies.

At pressures close to ambient $\left(\mathrm{p}=\mathrm{p}_{\mathrm{o}}\right.$ ) the equation above leads to the polarization curve shown in Fig. 110 (the values of the parameters used for the curve in are typical for state-of-the art fuel cell stacks produced by several manufacturers).

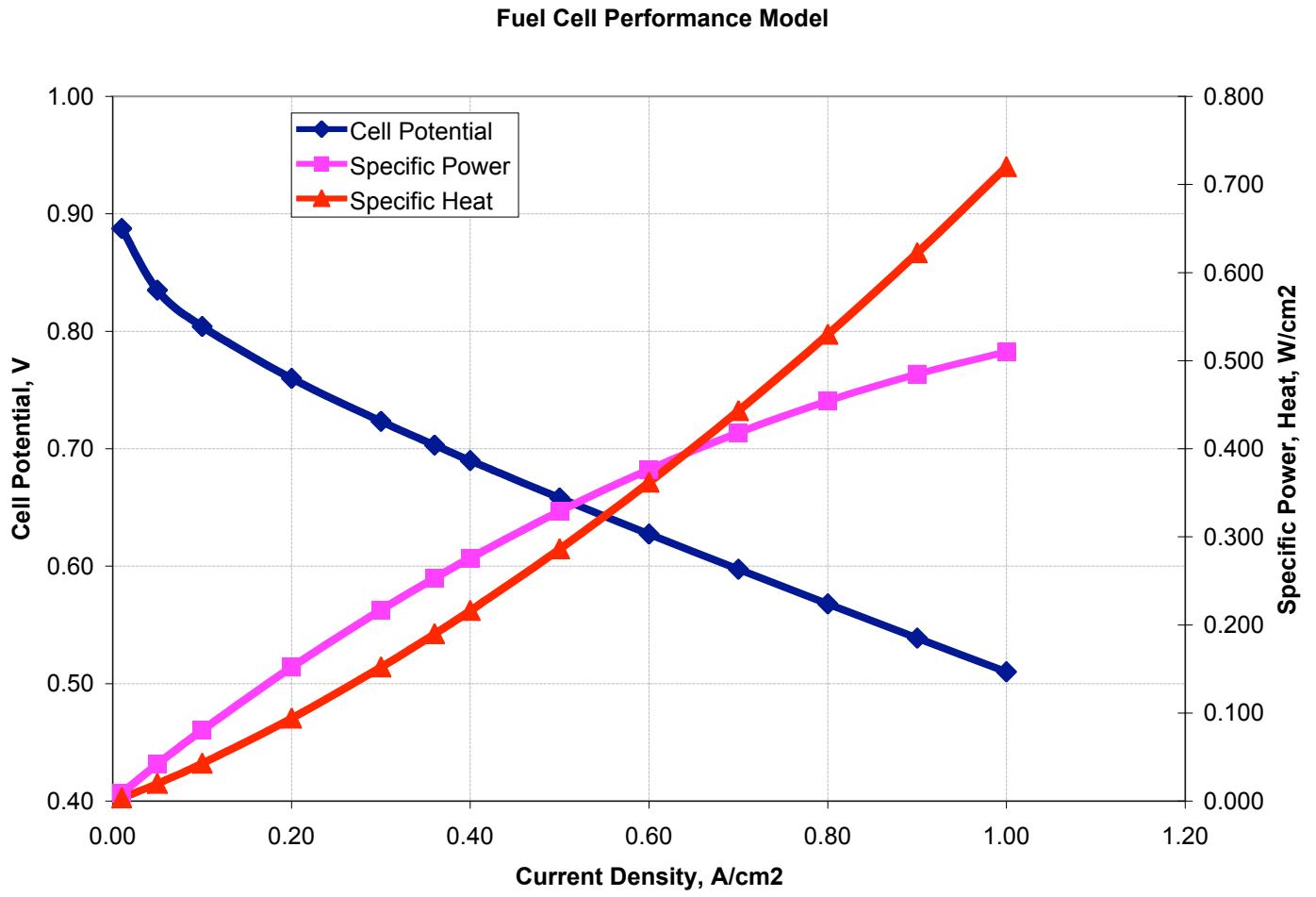

Fig. 110. Polarization curve for $E_{0}=0.95, b=0.06 \mathrm{~V} / \mathrm{dec}, \mathrm{R}=0.26 \square \cdot \mathrm{cm}^{2}, \mathrm{p}=\mathrm{p}_{0}$

For mechanical design purposes, the weight, volume, and power were computed as function of the active cell area, so that,
Weight:
$8-50 \mathrm{~kg} / \mathrm{m}^{2}$
Volume:
$29 \mathrm{~L} / \mathrm{m}^{2}$
Design Power (0.7 V/cell): $2.8 \mathrm{~kW} / \mathrm{m}^{2}$
Peak Power (0.6 V/cell): $\quad 3.8 \mathrm{~kW} / \mathrm{m}^{2}$ 
Note that the huge weight range was due to a wide range of technologies used by different manufacturers.

The volume figure assumed that there was a cooling cell (heat exchanger) integrated with every power producing cell, so the cell thickness was roughly $0.3 \mathrm{~cm} /$ cell. We could expect half that value for water cooling, but the heat exchanger would have to exist somewhere.

\section{Fuel Requirement}

When dealing with fuel cell and hydrogen consumption, it was convenient to put everything in terms of current because then we would just have to make sure that we had 1 electron for each hydrogen atom. I.e., we knew that $1 \mathrm{Amp}=1 \mathrm{C} / \mathrm{s}$ and the charge on an electron was 1.6e-19 C, and that, in a fuel cell, we generate 6.022045e 23 electrons per mole of Hydrogen atoms, so that we would have $1 \mathrm{Amp}=1 \mathrm{Coul} / \mathrm{s}=1$ atoms of H/1.6e-19 $\mathrm{s}=1 \mathrm{~mole}$ of $\mathrm{H} /(1.6 \mathrm{e}-19 * 6.022045 \mathrm{e} 23) \mathrm{s}=1.04 \mathrm{e}-5 \mathrm{moles}$ of $\mathrm{H} / \mathrm{sec}=1.05 \mathrm{e}-5 \mathrm{~g} / \mathrm{s}=0.0378 \mathrm{~g} / \mathrm{hr}$, so hydrogen consumption is, $0.0378 \frac{\mathrm{g}}{\mathrm{Ah}}$, or $10.5 \frac{\square \mathrm{g} / \mathrm{s}}{\mathrm{A}}$.

\section{$\underline{\text { Air Requirement }}$}

Air contains $21 \%$ oxygen by volume. Since every molecule of oxygen exchanges four electrons during the electrochemical process occurring in the fuel cell reaction, a flow of $2.59 \mathrm{e}-6 \mathrm{moles} / \mathrm{s}$ of oxygen gas at $100 \%$ utilization was needed to support $1 \mathrm{~A}$ of current per cell. This translated into $1.23 \mathrm{e}-5 \mathrm{moles} / \mathrm{s}$ of air at the same conditions.

Unfortunately, the presence of nitrogen and other inert gases in the air stream made it impossible to utilize all the oxygen from air, and a utilization of 50 to $30 \%$ of the inlet oxygen gave optimal performance. The minimum air-flow at $50 \%$ utilization would then be $2.47 \mathrm{e}-5 \mathrm{moles} / \mathrm{s}$ of air.

Another complication of the mass balance on the oxygen side of the fuel cell came from the need to remove the water produced during fuel cell operation. Almost all the water is produced on the oxygen (air) side of the fuel cell, and it is of paramount importance for stable operation to maintain balanced water removal. On the other side, when too much water is removed from the fuel cell - the membrane loses its conductivity and the fuel cell performance rapidly deteriorates.

\section{Chemistry, Cooling}

The fuel cell converts the chemical energy of the reaction of hydrogen with oxygen into electrical energy and heat, while producing water.

$$
\mathrm{H}_{2} \mathrm{O}_{2} \square \mathrm{H}_{2} \mathrm{O}+\mathrm{Q}
$$

$\mathrm{Q}=\mathrm{W}+\mathrm{q}$

$\mathrm{W}=\mathrm{E}_{\text {cell }} \mathrm{I}-$ electrical power

$\mathrm{Q}=\left(\mathrm{E}_{\mathrm{tn}}-\mathrm{E}_{\text {cell }}\right) \mathrm{I}-$ heat flux

Depending on the state of the water exiting the fuel cell (liquid or vapor), the amount of heat needed to be dissipated by the cooling system differs. When all the product water is removed 
from the cell in liquid form then $\mathrm{E}_{\mathrm{tn}}=\mathrm{E}_{\mathrm{hhv}}=1.48 \mathrm{~V}$. When the product water exits the fuel cell with the outlet stream of air in a vapor form, $\mathrm{E}_{\mathrm{tn}}=\mathrm{E}_{\mathrm{lhv}}=1.23 \mathrm{~V}$ at standard conditions.

\section{Thermal Model}

For our component model purposes it is assumed that the fuel cell was at constant temperature and pressure and that the product water exited the fuel cell in the form of vapor. The heat generation for each cell is computed using the difference between the lower heating value voltage, and the cell voltage via the following equation:

$$
q=(1.23 \square \mathrm{E}) \mathrm{i}
$$

We further assumed that the heat carried by the exiting product air stream, and the hydrogen stream was negligible, and that all the heat was dissipated through the fuel cell cooling loop. Figure 111 represents the steady state model of the fuel cell. The data in Table 23 gives the calculated flows and generated power and heat for a fuel cell stack with 20 cells of $200 \mathrm{~cm}^{2}$ active area, assuming that $100 \%$ of the supplied hydrogen was used and $50 \%$ of the oxygen from the supplied air was consumed in the reaction.

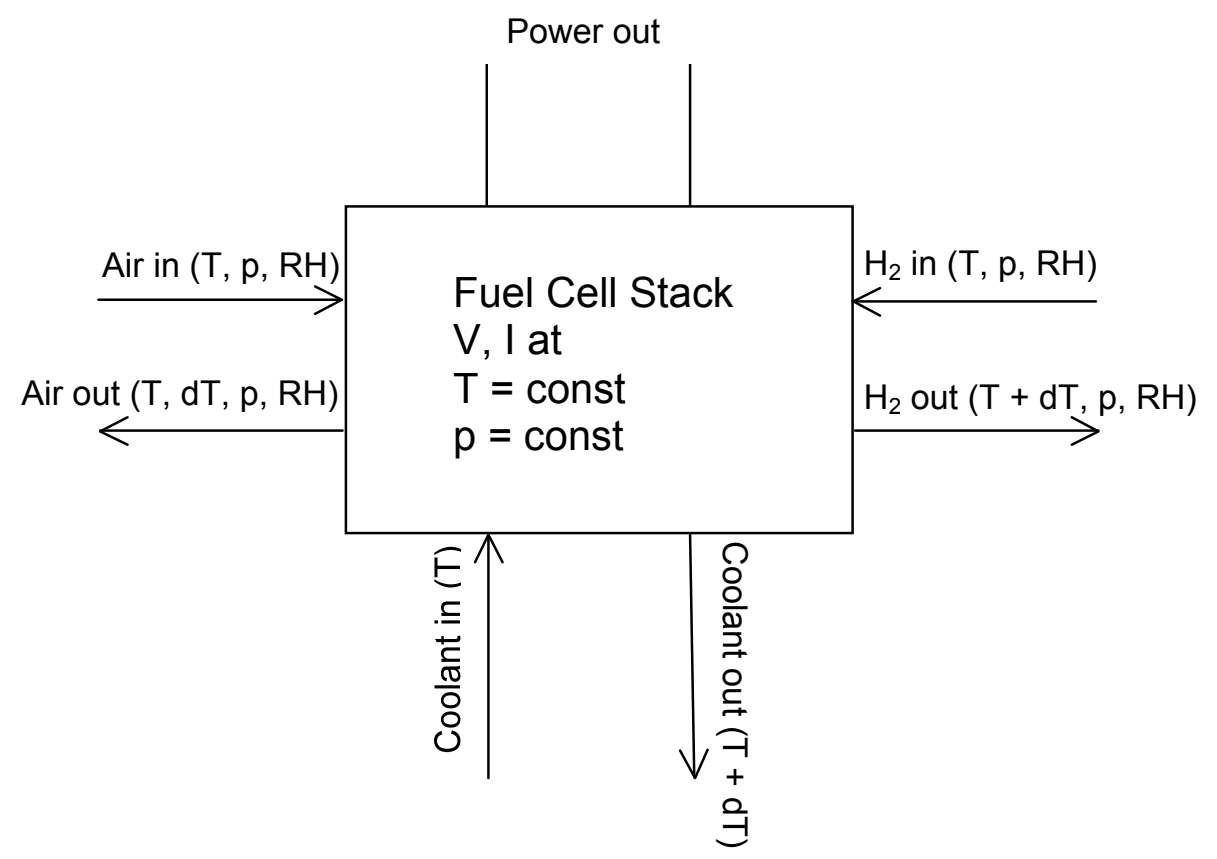

Fig. 111. Steady state model of the fuel cell stack. 


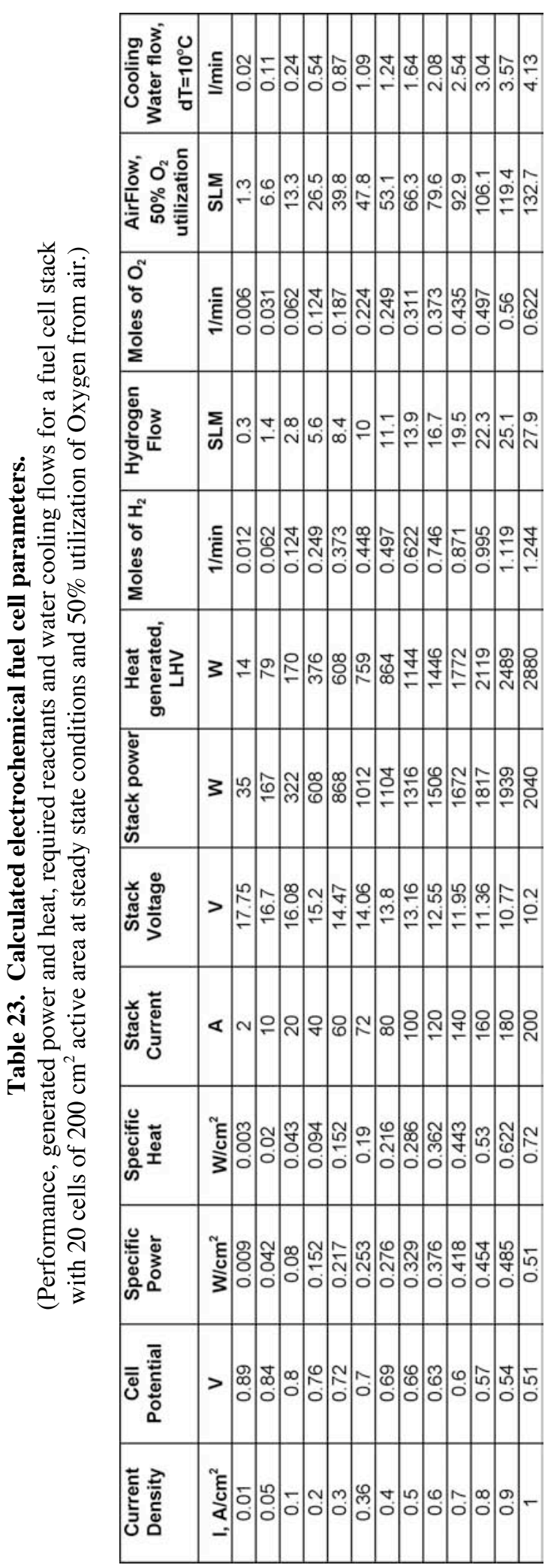




\section{Testing}

A fuel cell stack with the necessary auxiliary support equipment was procured for this project from H-Power. We verified the performance of the fuel cell system using the experimental setup shown on Fig. 112. The source of hydrogen for these experiments was a pressurized tank. The pressure supplied to the fuel cell was further regulated down to $20 \mathrm{psig}$. The system had an internal pressure regulator that further reduced the pressure supplied to the fuel cell to less than 5 psig. The hydrogen side of the fuel cell operated in a dead-end mode with periodic purges, necessary to vent any inert impurities and water accumulating during fuel cell stack operation. The air is supplied to the fuel cell using a pump, which was internal to the system. A microcontroller, supplied with the system, monitored internal temperature, pressure, fuel and oxidant flows. The fuel cell system was supplied with eight AAA size alkaline batteries for bootstrapping the system during start-up. An ABC-150 was used as a load for the performance evaluation. The ABC-150 is a very versatile computer controlled power processor, designed for testing all types of energy storage systems. It has the capabilities to deliver very fast transients, in power, current or voltage modes. Its internal data acquisition system can collect current and stack voltage data at $0.1 \mathrm{~s}$ intervals.

The fuel cell start-up is quick. The system is capable of delivering $80 \%$ of its full rated power in less than one minute.

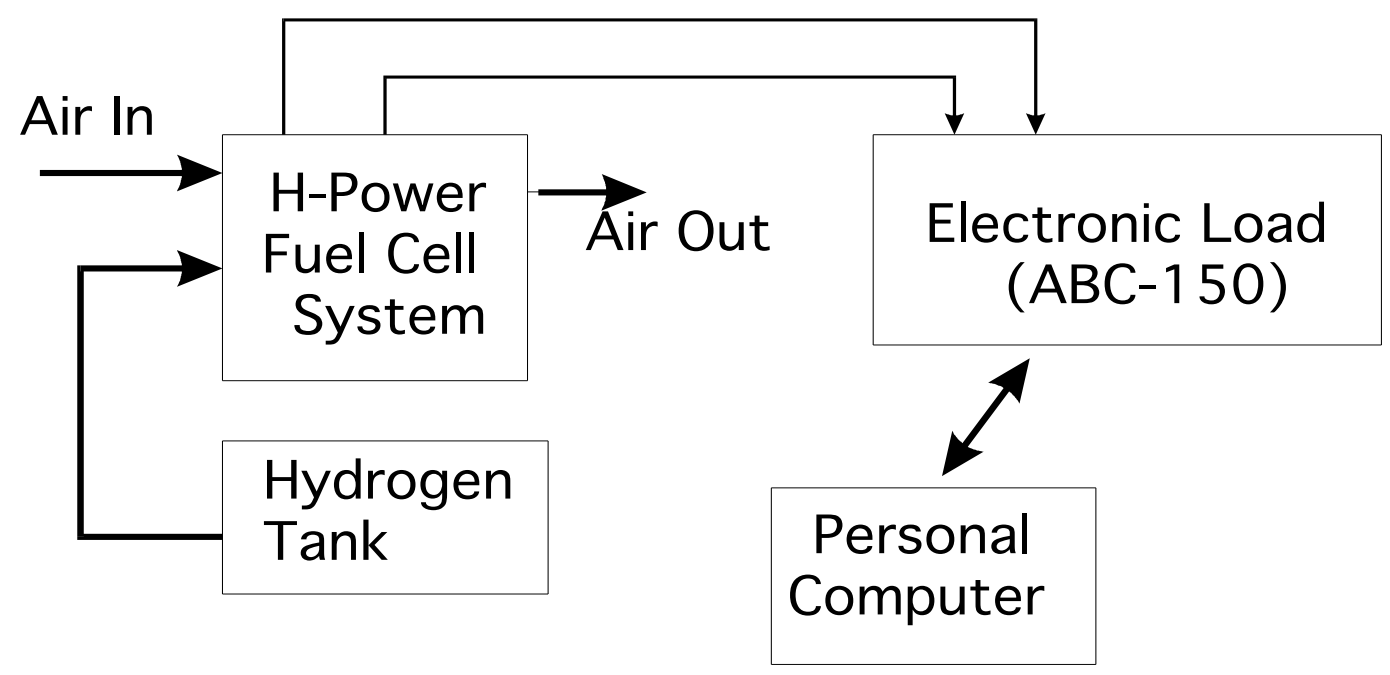

Fig. 112. Schematic diagram of the experimental set-up.

The data from the steady state performance of the fuel cell system are presented in Fig. 113. The data were recorded after the stack was operated in a steady state mode for 30 minutes in order to bring it to optimal conditions. The filled triangles represent stack voltages after 2-minute dwell time at a given stack current. The filled rhomboids are the measured stack voltages with five second dwell time at each current. The solid lines are polynomial approximations of the stack voltage and delivered power as a function of stack current. An unusual instability of the stack performance was observed repeatedly in the range of current densities between 5 and $8 \mathrm{~A}$. 


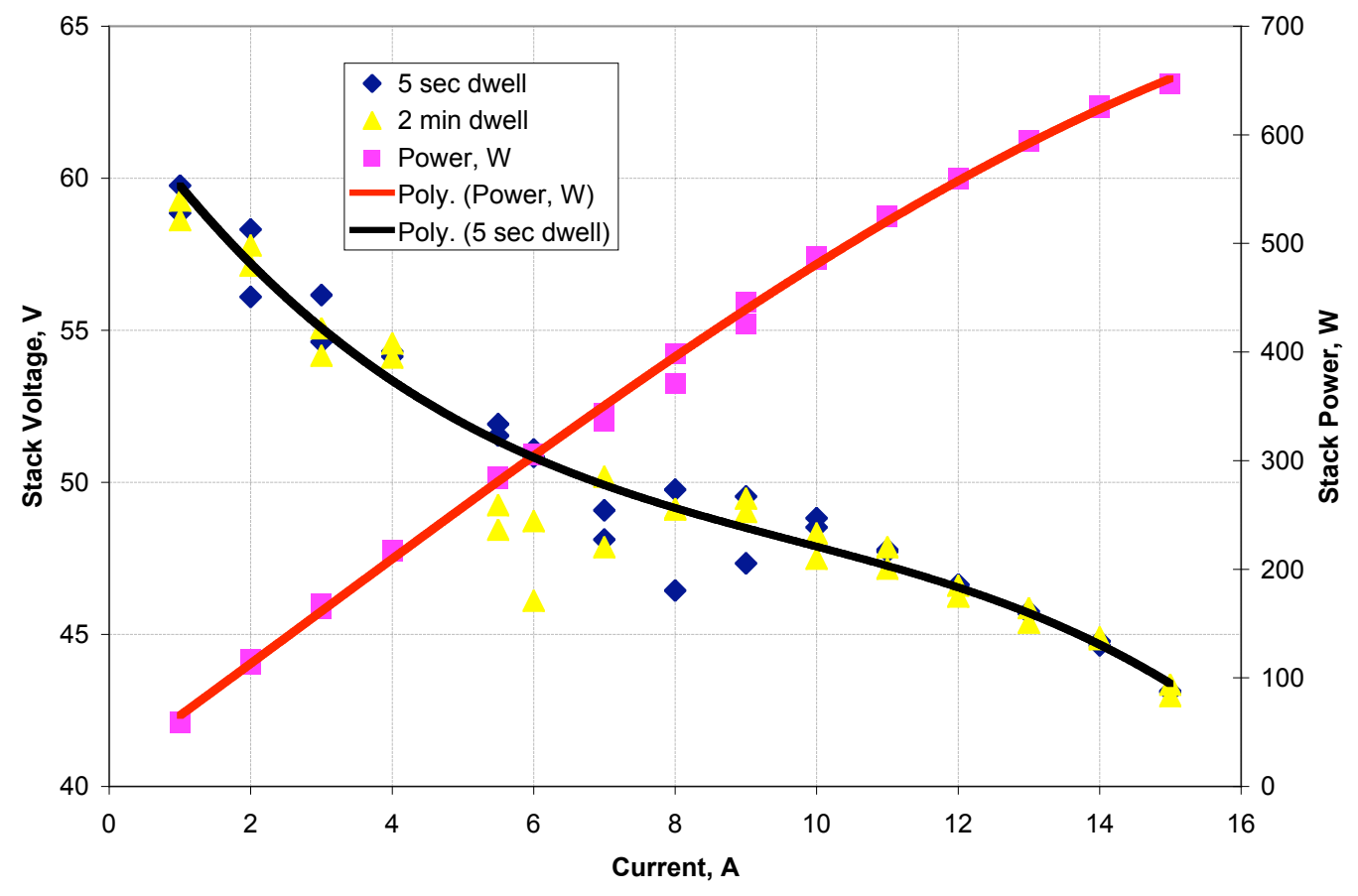

Fig. 113. Typical measured dependence of stack voltage.

(As a function of stack current at optimal system conditions.)

After we convinced ourselves that the fuel cell system performance was very close to the performance described in the technical manual we proceeded with an effort to characterize the system with pulse loads. After a short period for fuel cell conditioning we requested stack current of $2 \mathrm{~A}$ for the low power level and $15 \mathrm{~A}$ for the high power level. The length of the high power level pulse was set at $20 \mathrm{~s}$. Data for voltage and current were collected at $100 \mathrm{~ms}$ intervals. From the graph in Fig. 114 it is evident that the load following capabilities of the fuel cell in this mode are excellent. To illustrate better this point the first and last pulses are presented in a larger scale graph in Fig. 115. One can see that the transition between operation at $100 \mathrm{~W}$ and $650 \mathrm{~W}$ is instantaneous and there is no deterioration in performance (see Fig. 116 for comparison).

Since the exact power profile for the exoskeleton as a function of time is not known at this time we decided to verify one more mode of pulsed operation - with much longer pulses. Results from an experiment with 3 minute long power pulses are presented in Fig. 117 and Fig. 118. It was evident that even with the long pulse the fuel cell system was able to maintain steady performance at the maximum power level.

It is important to note that the behavior of the fuel cell stack in pulse mode was strongly dependent on the method of fuel and oxidant delivery to the stack. For these experiments hydrogen was readily available, and the air pump was also operating constantly. 


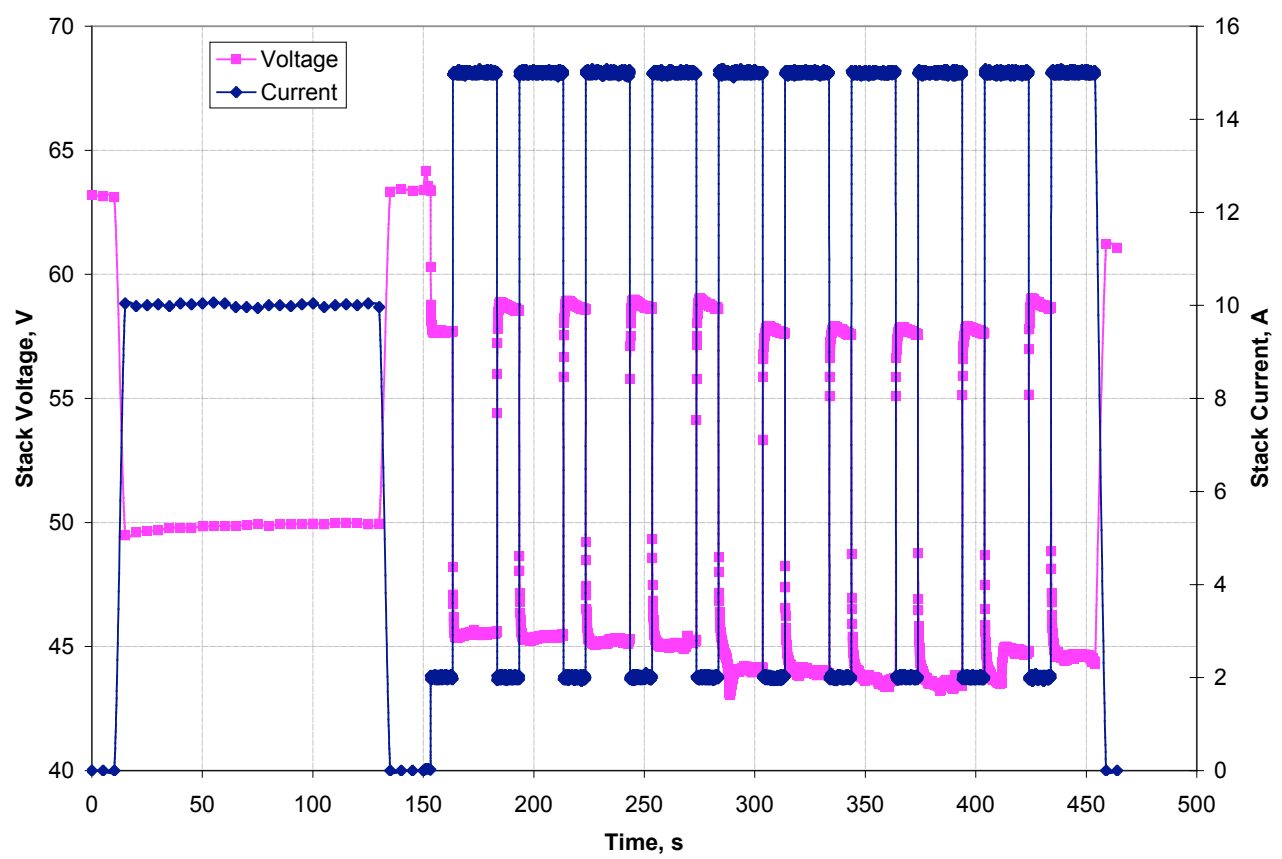

Fig. 114. Stack voltage and current as a function of time. Pulse width $20 \mathrm{~s}$.

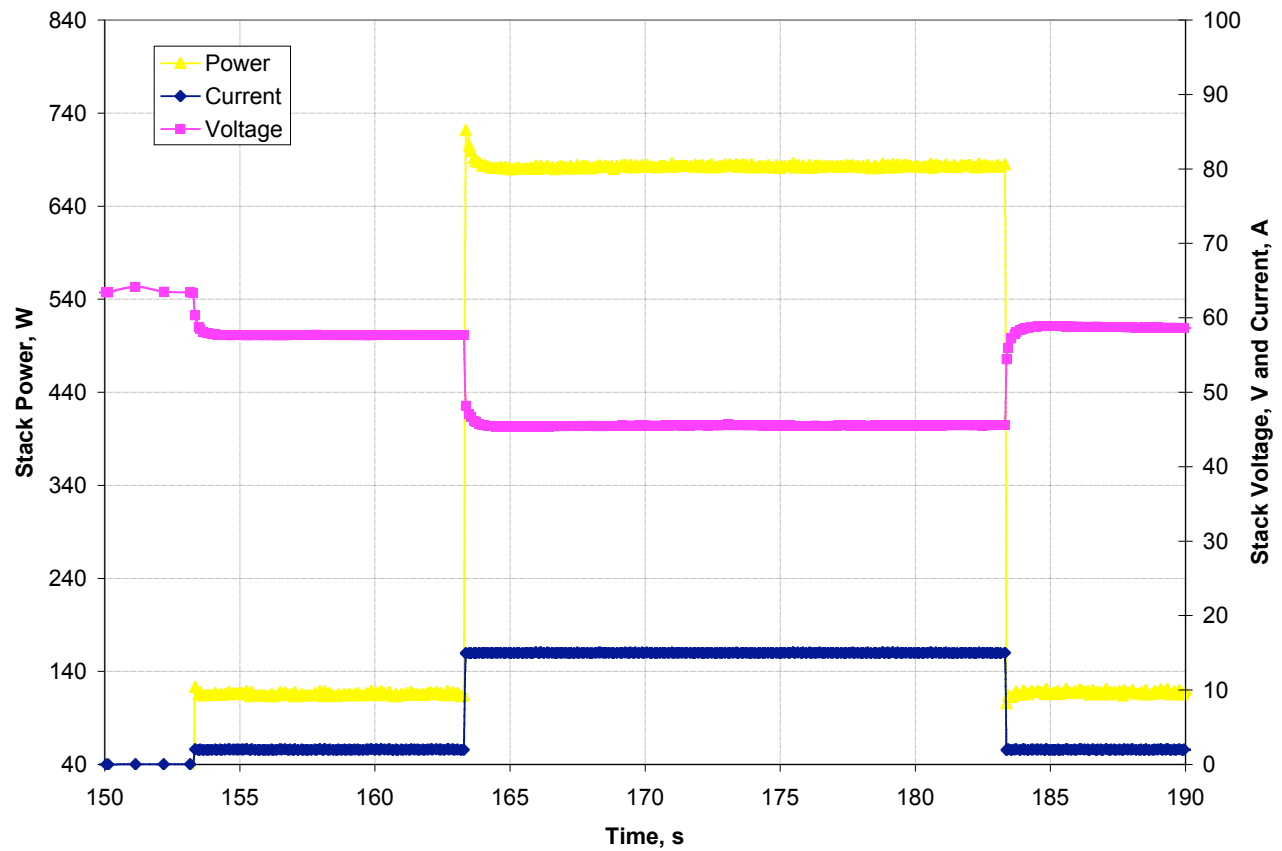

Fig. 115. Stack power, voltage and current as a function of time. Pulse width $20 \mathrm{~s}$

(Beginning of the pulse sequence.) 


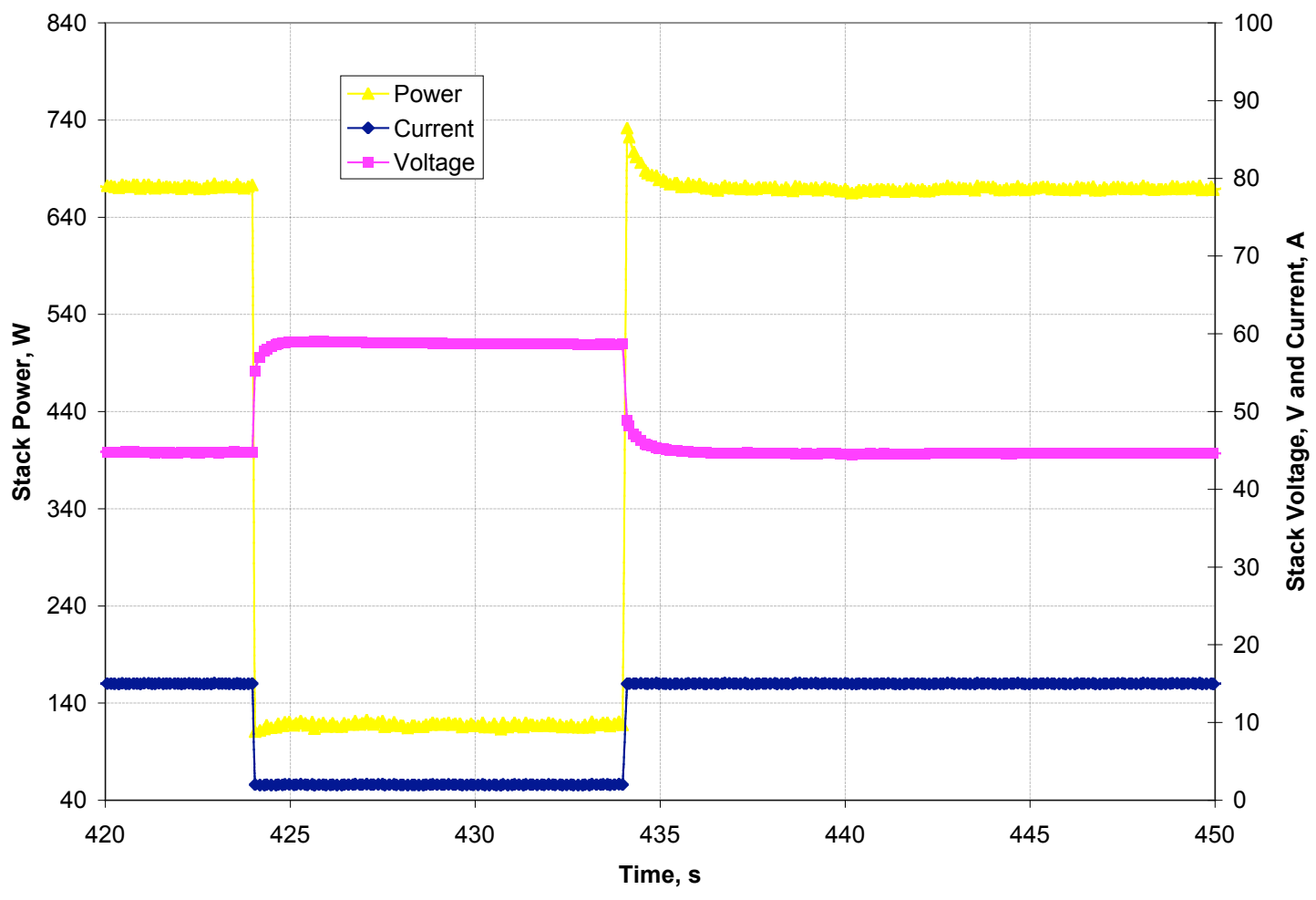

Fig. 116 Stack power, voltage and current as a function of time. Pulse width $20 \mathrm{~s}$. (End of the pulse sequence.)

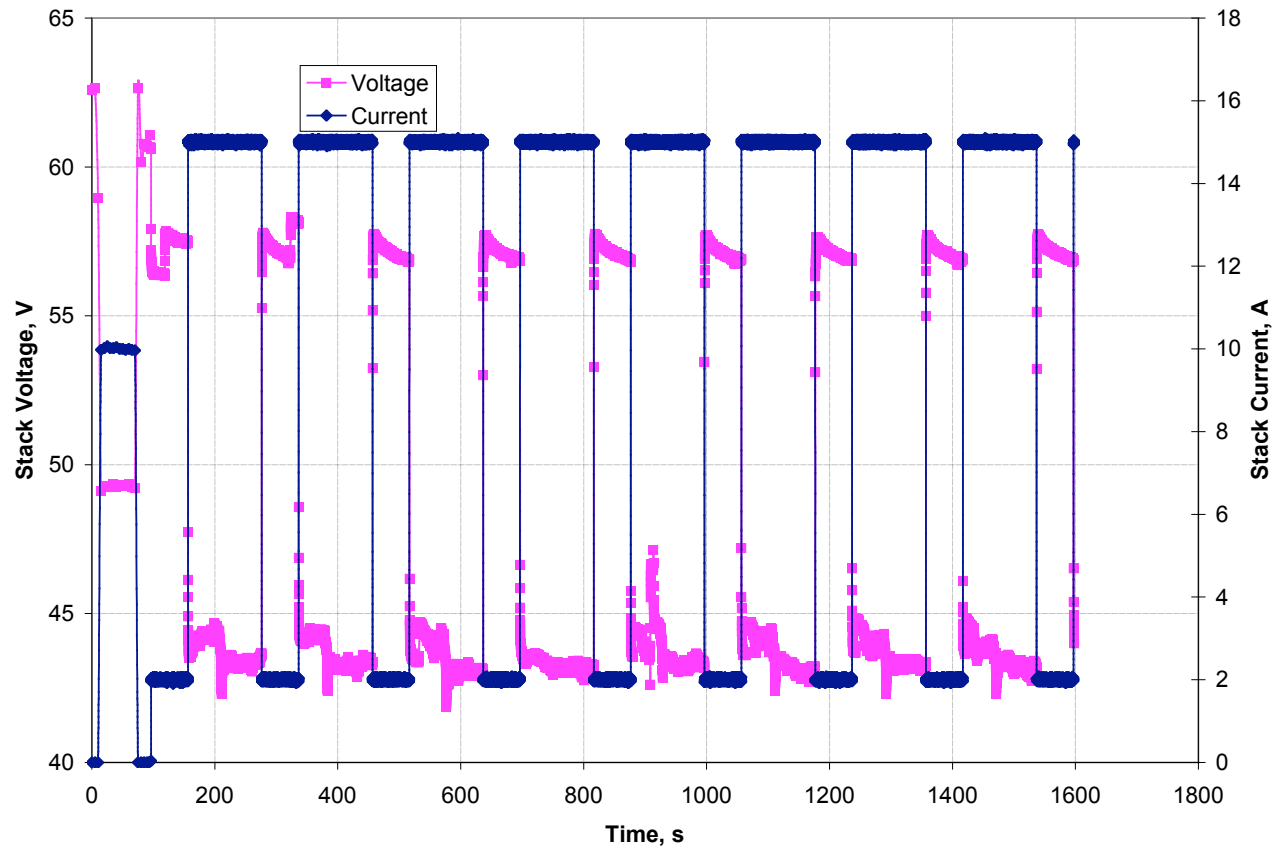

Fig. 117. Stack voltage and current as a function of time. Pulse width $180 \mathrm{~s}$. 


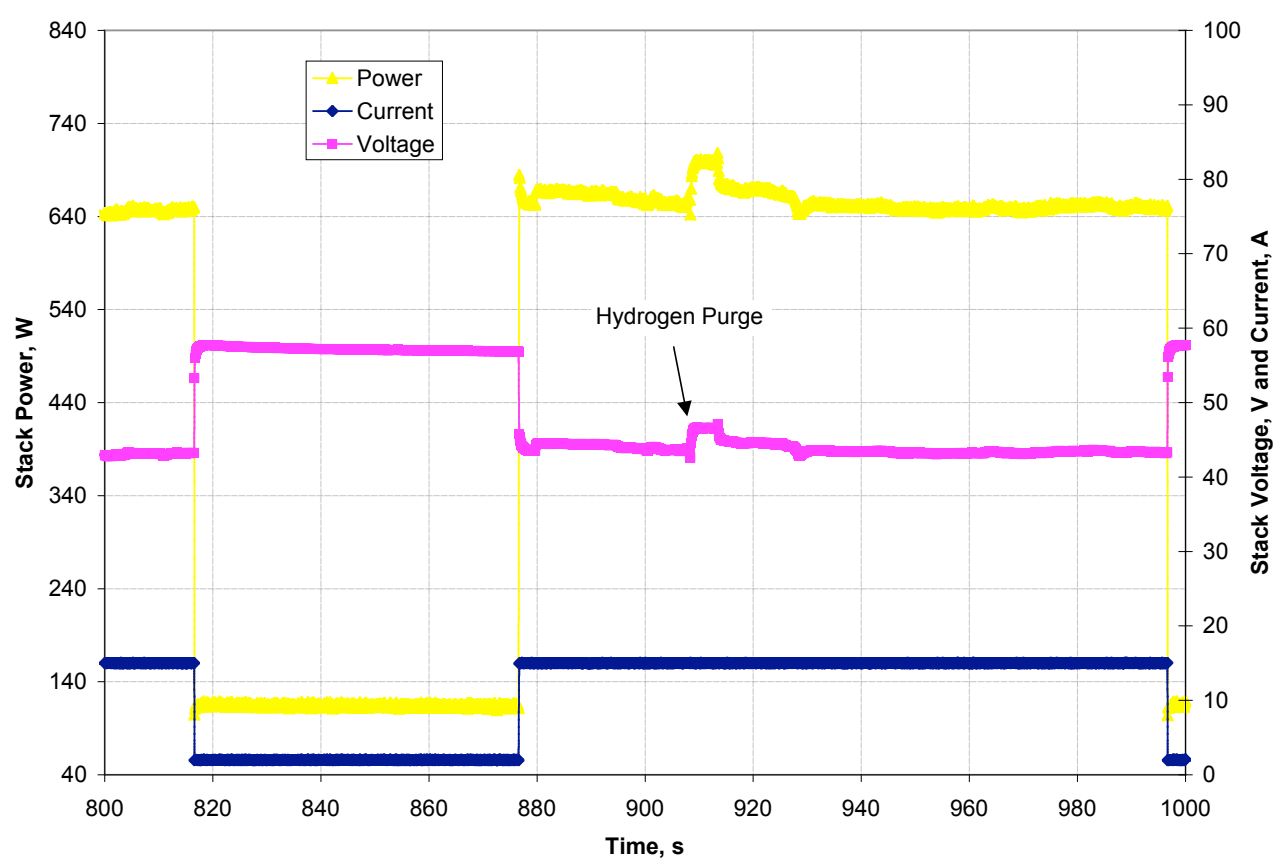

Fig. 118. Stack power, voltage and current as a function of time. Pulse width 180s (Single pulse.)

\section{Results: Digital Data Acquisition and System Control}

\section{Layout}

The data acquisition and control system was based on the Battery System Controller (BSC) developed at AeroVironment over several years, and used in numerous energy storage systems based on battery packs, fuel cells, microturbine, flywheels etc. A schematic of the system architecture is shown in Fig. 119. The BSC monitors all cell voltages of the fuel cell stack, the transient handler pack voltage and temperature, the FC current, the transient handler current and the temperatures of the hydrogen generator. It sends commands to the cooling fans, the air compressor, the solution pump of the hydrogen generator and the solenoid valves. It also output all the data via a serial port for monitoring and recording.

Several software and hardware modifications and enhancements were required in order to meet the specific objectives for the exoskeleton energy storage system. 


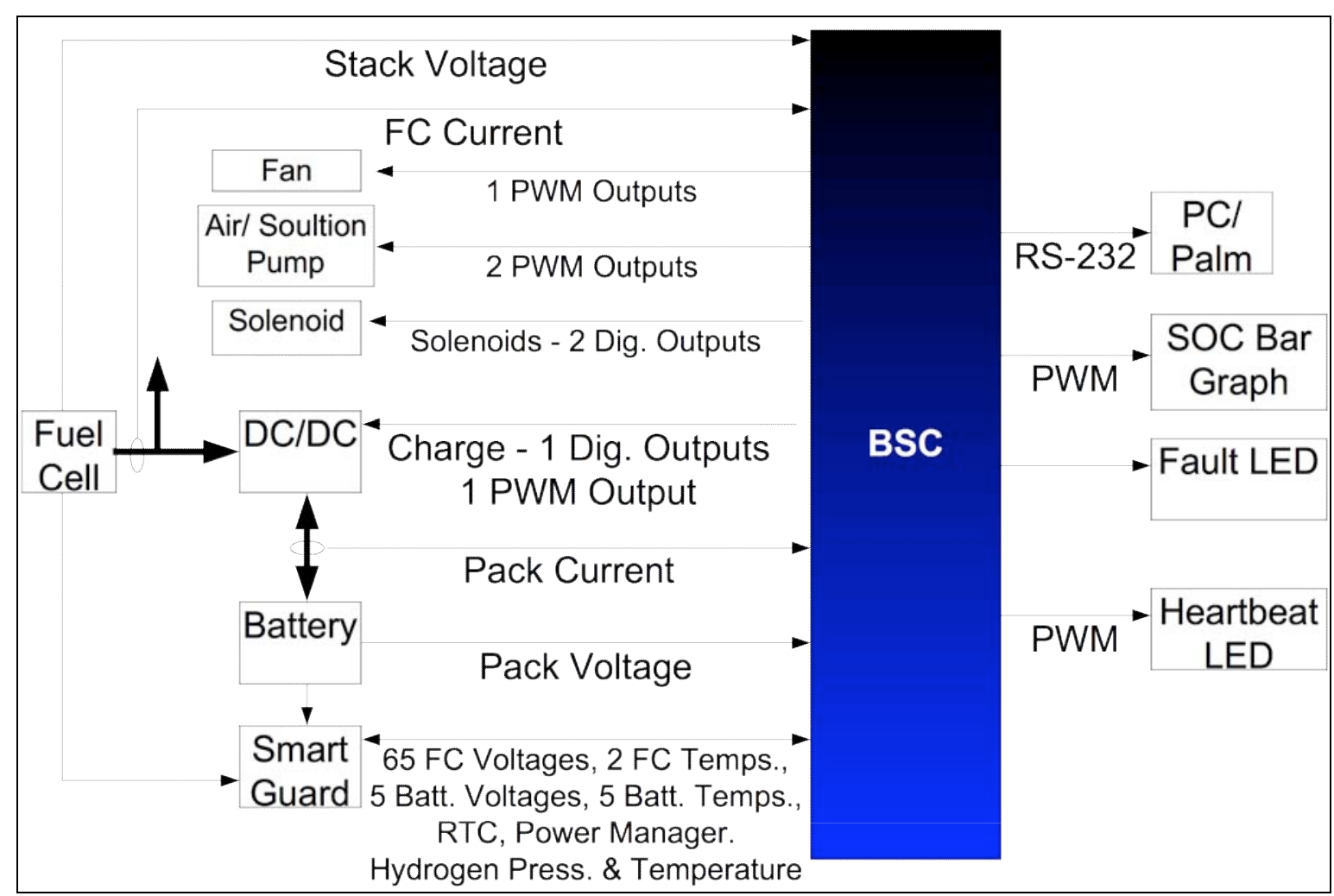

Fig. 119. Energy storage system control diagram.

\section{Results: Hybrid System Configuration}

With the components exercised, our next step was to see if the main components of the hybrid system could be scaled to an established duty or known range of operating conditions for future exoskeleton work.

\section{Selection}

An operating voltage of 48VDC was established to be the best voltage to allow for the hybrid system to be controlled. From this operating voltage a system control concept was developed and a control circuit was designed around this concept. Based upon our previous test data from the battery testing, it was decided that the Panasonic NiMH batteries were the best choice for the transient handler system. From there the system was broken into three main parts:

1. Fuel Cell System - the H-Power Fuel Cell, pressure regulator, and high pressure hydrogen supply

2. Transient Handler System - DC/DC converters, control logic circuit, diodes, NiMH battery sticks, fuses, terminal block, and thermal switch

3. Load System - an on/off switch, timer relay, relays, $150 \mathrm{~W}$ load for continuous load to system, $1850 \mathrm{~W}$ load on relays as a pulse load. 
The control concept, as shown in Fig. 120 for the hybrid configuration of the fuel cell system was as follows:

1. As a pulse load that is greater than the power capability of the fuel cell system is sensed by the control circuit of the transient handler system, it "turns on" the DC/DC converters. This then pulls power from the transient handler system and fuel cell system in parallel.

2. This continues until the pulse load stops, the transient handler system is to a level that requires it to be recharged (battery cell voltage $<=1.0 \mathrm{VDC}$ ), or the transient handler system is to a level that it is considered fully charged (battery cell voltage $>=1.5 \mathrm{VDC}$ ).

3. At this point the fuel cell system handles the entire load until the pulse load exceeds the fuel cell system power and the cycle of maintaining the transient handler system's state of charge starts over. In this way the fuel cell always operates at rated power and system controller regulates the transient handler voltage in a way that it will not damage itself and also maintain a high state of charge.

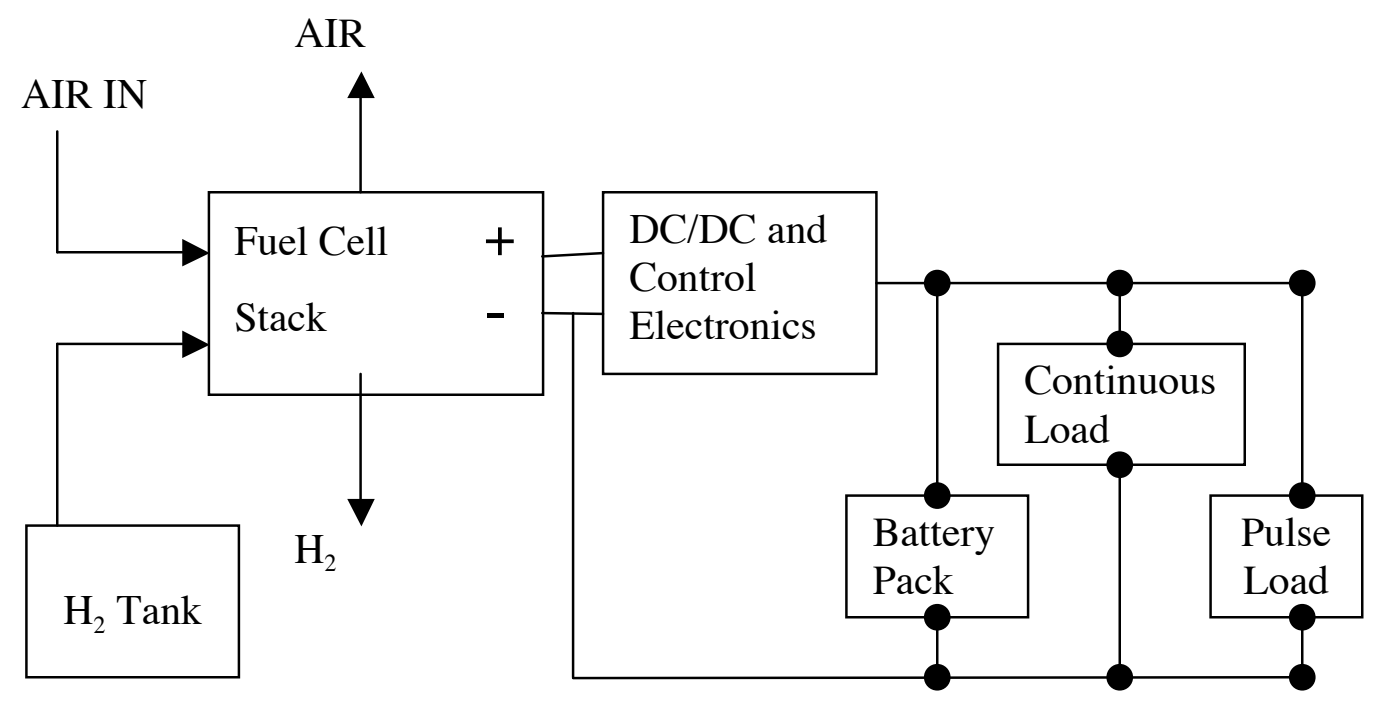

Fig. 120. Hybrid fuel cell system diagram.

The Transient Handler parts, shown in Fig. 121 consisted of the following:

10 NiMH cell "sticks" (7 elements in a "stick", 5 "sticks" in a series, 2 parallel groups) DC/DC converter mounted on an Aluminum heat sink Transient Handler control circuit Components for circuit measurements and protection Thermal switches mounted to sticks for cell protection 


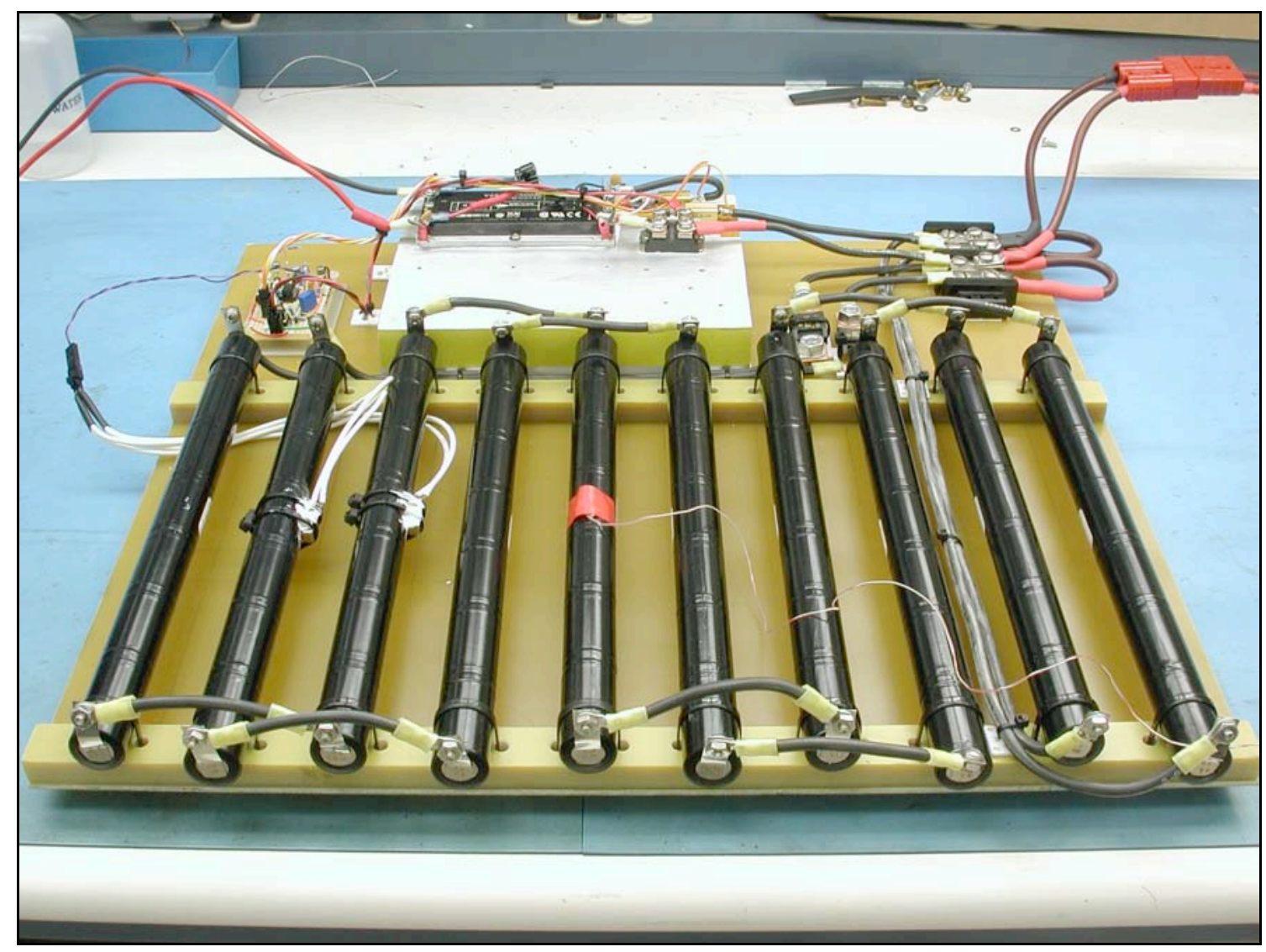

Fig. 121. Transient handler system.

The Pulse Load parts (Fig. 122) consisted of the following:

$150 \mathrm{~W}$ continuous load (halogen automotive light bulbs)

On/off switch

Variable time delay relay to control pulse load relays

Load relays for pulse loads

$1850 \mathrm{~W}$ pulse load (off-road high intensity light bulbs)

Instrumentation for the testing consisted of clamp-on style ammeters, voltmeters, and thermocouples. The testing was videotaped with a clock in the background to show the duty cycle times. The pulse loads were also run at varying times to observe the transient handling capabilities of the system. To have the load be "visual" high intensity automotive lights were chosen to be the load so that the load and the representative duty cycle tested could be observed. Fig. 123 presents the bench test setup that was used to check the system. 


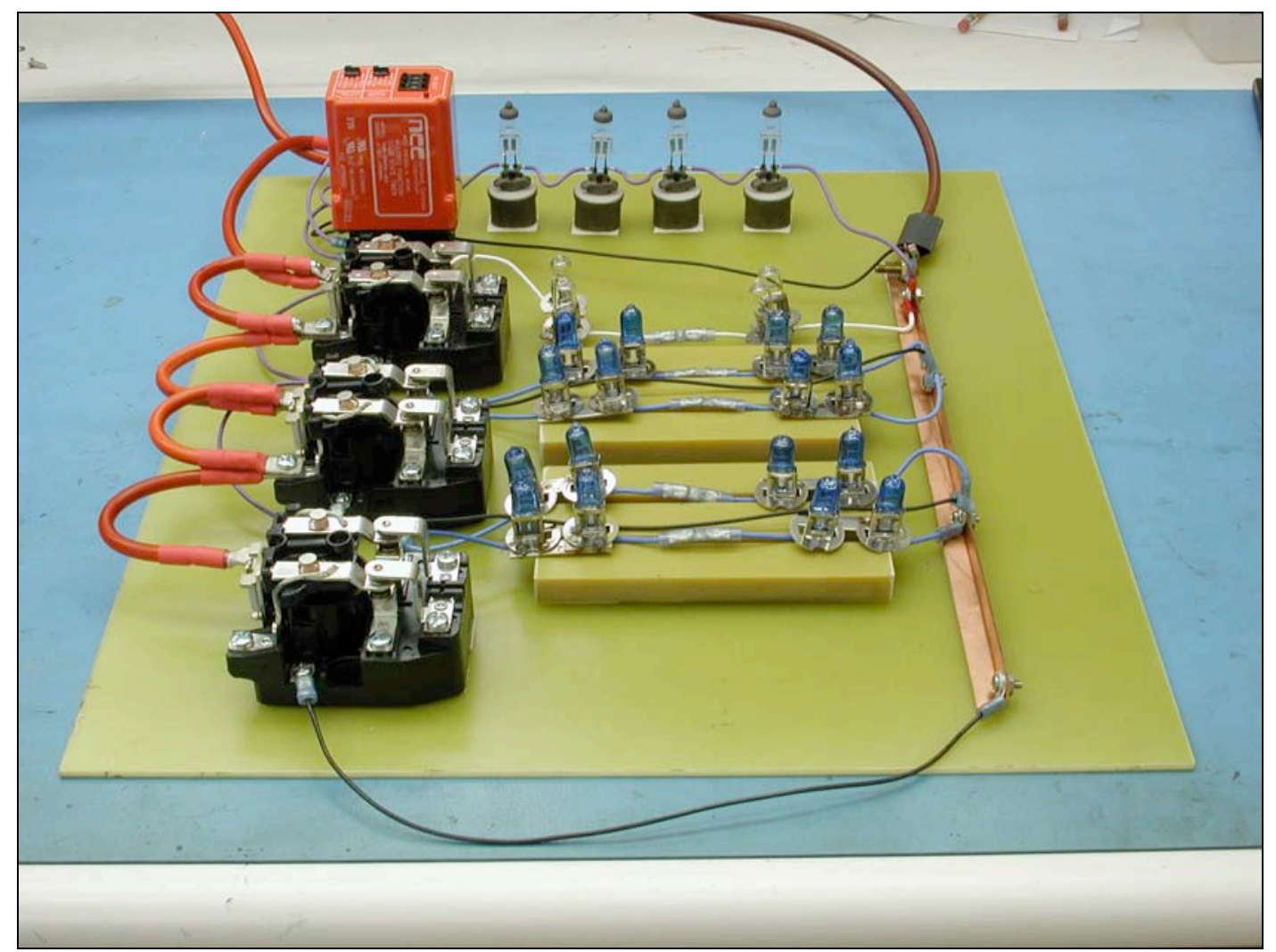

Fig. 122. Pulse load system.

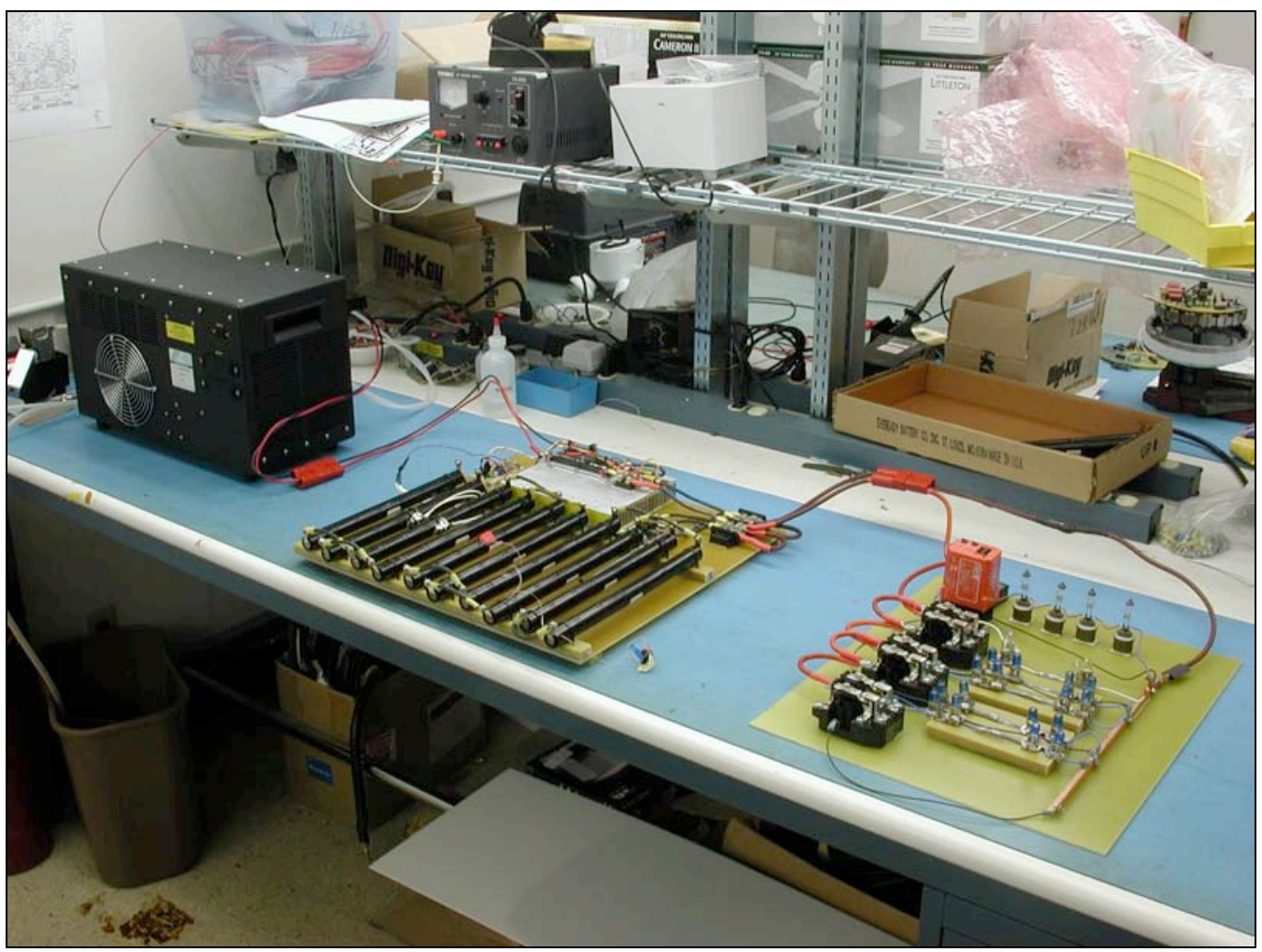

Fig. 123. Hybrid fuel cell system bench test set-up. 


\section{Testing}

The following tests were performed:

\section{LOAD/CYCLE}

150 W Continuous (Fuel Cell only)

$150 \mathrm{~W}$ continuous $\mathrm{w} / \sim 400 \mathrm{~W}$ pulse load

\section{DURATION/CONCLUSION}

\section{PASS}

PASS - no affect from varying pulse@ listed time cycles (Fuel Cell only)

$.5 \mathrm{sec}$ pulse load

$1 \mathrm{sec}$ pulse load

$2 \mathrm{sec}$ pulse load

9 sec pulse load

$150 \mathrm{~W}$ continuous w/ 1850 W pulse load@ PASS - note varying duration listed time cycles (Fuel Cell and Transient Handler)

$.5 \mathrm{sec}$ pulse load

$\sim 13$ min. cycle

$1 \mathrm{sec}$ pulse load

$\sim 13$ min. cycle

$2 \mathrm{sec}$ pulse load

$\sim 12$ min. cycle

5 sec pulse load

$\sim 10$ min. cycle

9 sec pulse load

$\sim 9$ min. cycle

Time between cycles to recharge Transient Handler was about 15 to 18 minutes.

Results

Included in the Appendices of this report is a video demonstrating that the system was capable of handling the various input cycles listed below:

Validation of the system approach and its components

Transient response of Fuel Cell to loading

Transient Handler system capable of sustaining $2 \mathrm{~kW}$ pulse loads

Hybrid system capable of maintaining loads at a derived duty cycle.

\section{Hydrogen Generation and Control}

The hydrogen storage chemistry used for the Exoskeleton Power System was a solution of sodium-borohydride $\left(\mathrm{NaBH}_{4}\right)$ and water $\left(\mathrm{H}_{2} \mathrm{O}\right)$ that was then passed over a catalyst to initiate the production of hydrogen. The catalytic chamber for this project was supplied by ORNL and was proprietary in nature. The physical size of this catalytic chamber can be seen in the following Figs. 124 and 125. It is 1" in diameter, and approximately 6" in length. 


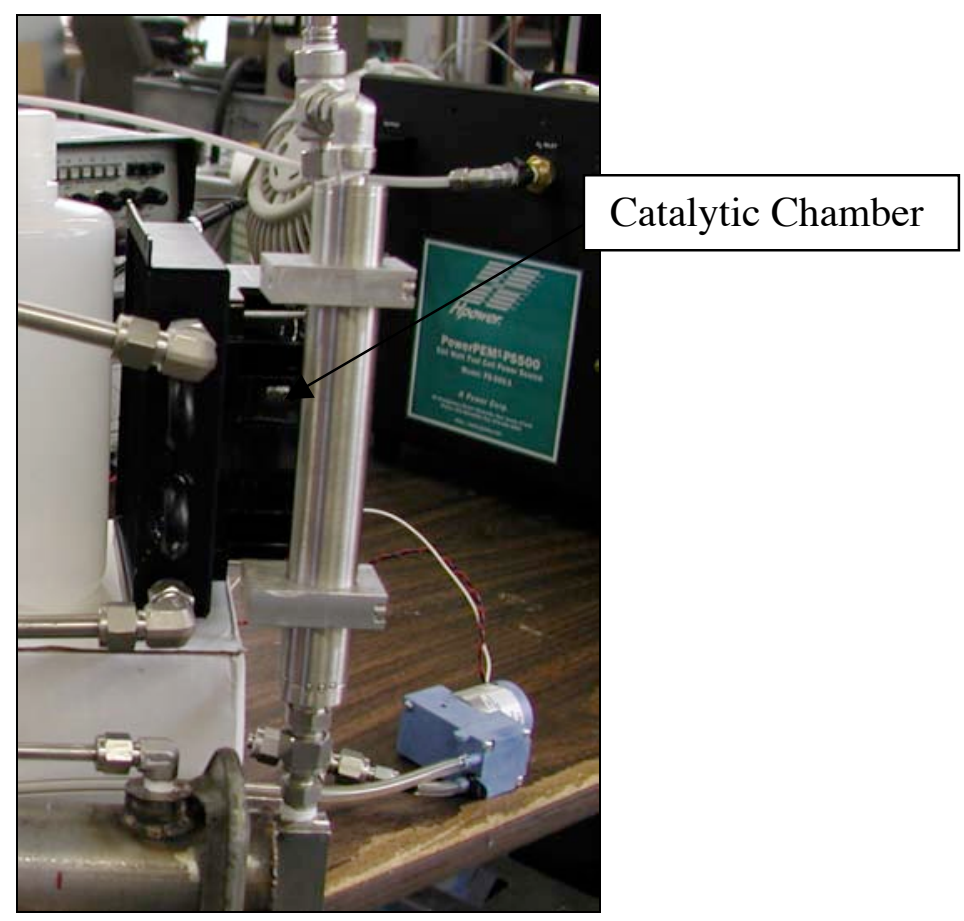

Fig. 124. The catalytic chamber.

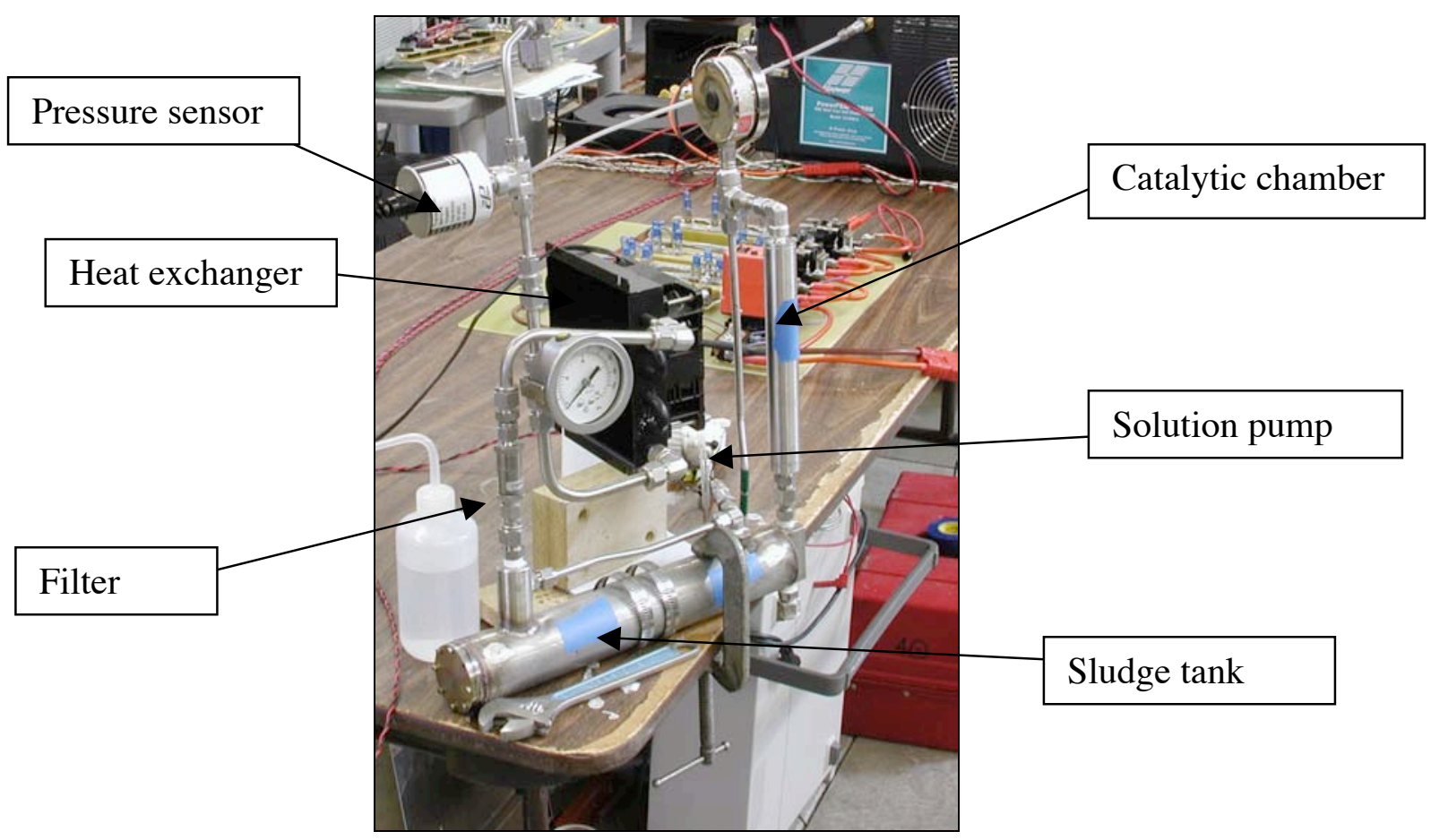

Fig. 125. System components.

ORNL also suggested that we use the solution of sodium-borohydride and water, based upon the percentage by weight of sodium-borohydride to the water. The recommended mixture start point was $17 \%$. If the percentage of $\mathrm{NaBH}_{4}$ were made much higher than $17 \%$, it would tend to clog the catalytic chamber after very short periods of time, and also demonstrate a significant amount 
of spontaneous hydrolysis. The chemical equation for the process as it is passed over the catalyst is shown below.

$$
\mathrm{NaBH}_{4}+2 \mathrm{H}_{2} \mathrm{O}=4 \mathrm{H}_{2}+\mathrm{NaBO}_{2}+74 \mathrm{~kJ} / \text { mole of heat }
$$

A spread sheet analysis tool was used to predict: (1) the amount of hydrogen required from a known duty cycle, (2) the effect of that duty cycle on the state of charge on the hybrid system, (3) heat rejected from the fuel cell and hydrogen generator, (4) water produced, (5) $\mathrm{NaBH}_{4}+2 \mathrm{H}_{2} \mathrm{O}$ solution needed, (6) sludge solution produced, and (7) air requirements for the PEM fuel cell stack.

From this we were able to estimate weights and sizes of a viable power system for the Exoskeleton system, and it gave us parameters and levels to test to during the operation of the system.

As an example, a $630 \mathrm{~W}$ PEM fuel cell demonstration system required the following:

$\begin{array}{ll}\text { Hydrogen flow rate } & 6.78 \mathrm{SLM} \\ \text { Air flow rate } & 33.92 \mathrm{SLM} \\ \mathrm{NaBH}_{4}+2 \mathrm{H}_{2} \mathrm{O} \text { solution flow rate } & 17.15 \mathrm{ml} / \mathrm{min} \\ \text { Individual fuel cell stack cell voltage } & 0.65 \mathrm{volts} \\ \text { Heat generated by fuel cell stack } & 563 \mathrm{~W} \\ \text { Heat generated by hydrogen generator } & 380 \mathrm{~W}\end{array}$

As we first started to develop the system, we used these parameters for sizing of system components. After operating the system we found that finite control of the solution pump for the hydrogen generation system was not really required. The system operated in a mode of "self regulation". As the solution pump fed solution to the catalytic chamber and hydrogen gas was produced, the pump stalled at a certain gas pressure, regardless of flow rate, and filled up the volume of sludge tank and tubing leading up to the hydrogen inlet of the fuel cell stack. The fuel cell then used up this hydrogen, the gas pressure dropped, the pump was no longer stalled, and solution would flow again. The system seemed to regulate itself to an operating pressure between 15-25 psig. The percentage of $\mathrm{NaBH}_{4}$ in the solution seemed to have a drastic effect upon system performance. At the base line of 17\%, we experienced a serious amount of hydrolysis, which had a large effect on the pump performance (i.e., pump cavitation due to hydrogen bubbles in the solution). When we made up solutions of $10 \%-13 \%$, we no longer experienced the cavitation problems, and the system seemed to operate very well.

We operated the power system multiple times for up to 30 minutes (at which point the system ran out of solution, and sludge tank storage). It would track whatever load was placed on the system with the light load bank. The fuel cell performance never seemed to be affected by, or have an effect upon the hydrogen generation system. More tests will have to be done to help optimize the system, but all in all, it tended to work very well, with very simple elements of control and monitoring.

Once the key subsystems were working properly, we added the system controller and the additional hardware to measure and control all system parameters. We also added the chemical 
hydrogen generation subsystem, prepared the sodium borohydride solution and started operating the system. The prototype system was ready to operate. The system starts with a push of button and is all self controlled and self contained. The picture in Fig. 127 illustrates best the overall concept. The PC is used for data display and recording only. The electric load is on the bottom shelf and is used for system testing. The fuel cell stack is an off the shelf system and no effort has been expended in reducing the size of the stack or the balance of plant components. The whole system is spread out and is not optimized for weight or size.
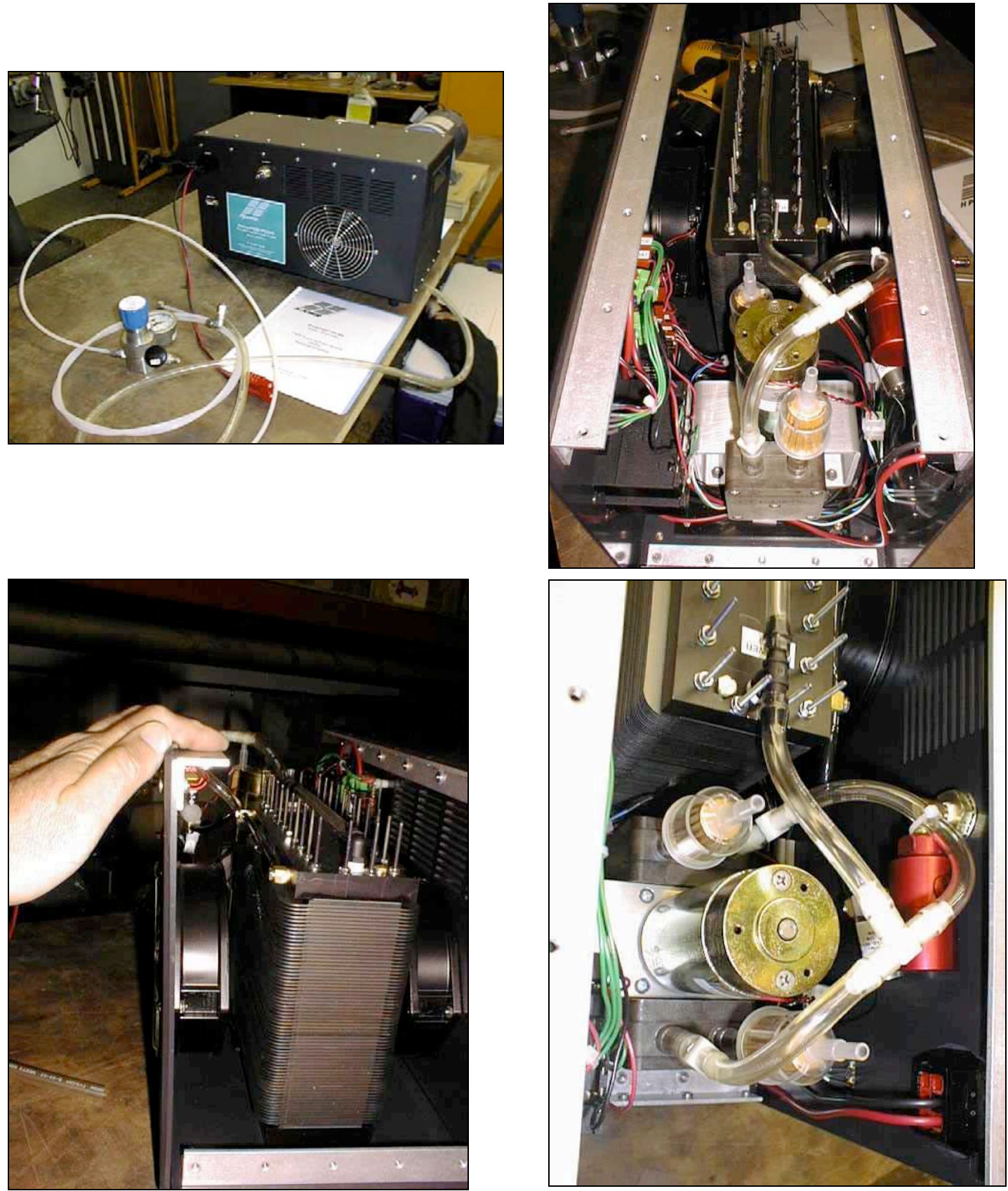

Fig. 126. H-Power fuel cell stack - nominal power $600 \mathrm{~W}$. 


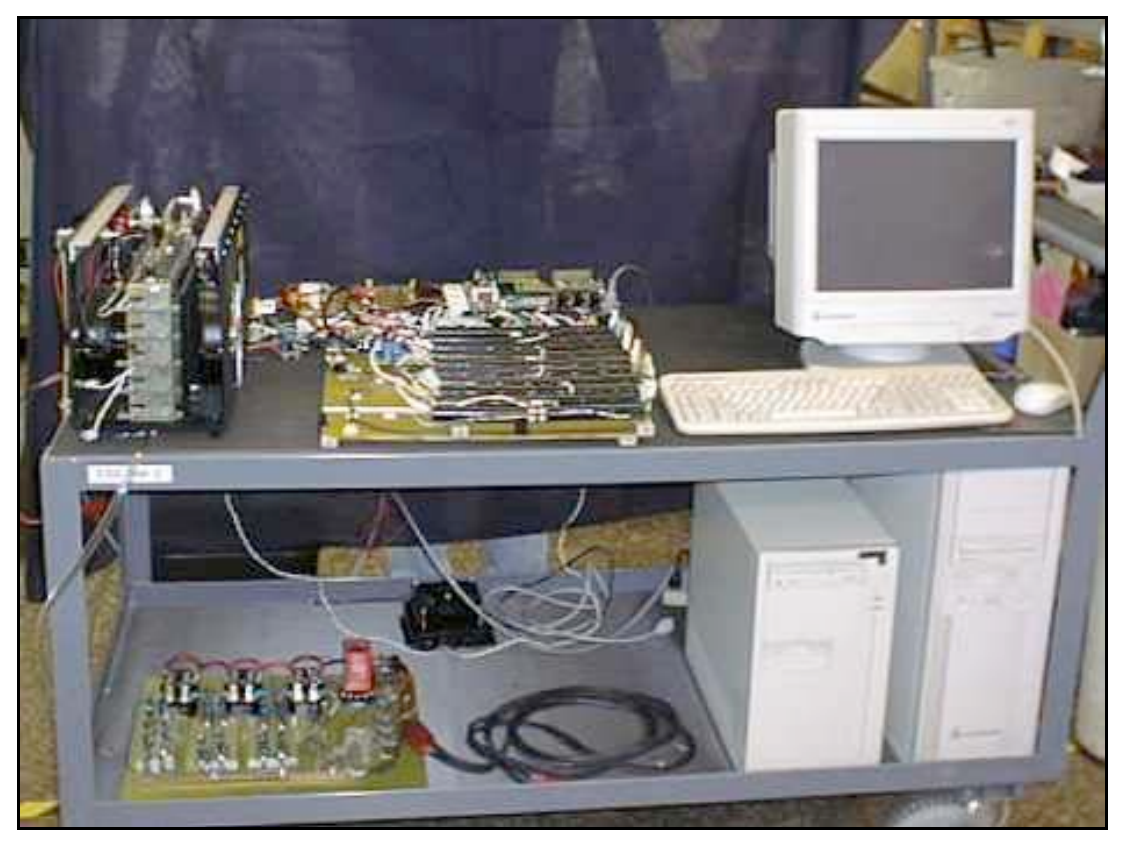

Fig. 127. Overall system.

The data in Fig. 128 illustrate how the system can deliver peak power to the electric load as needed. When the transient handler state-of-charge dips below $20 \%$ the fuel cell starts charging it, whenever there is excess power available.

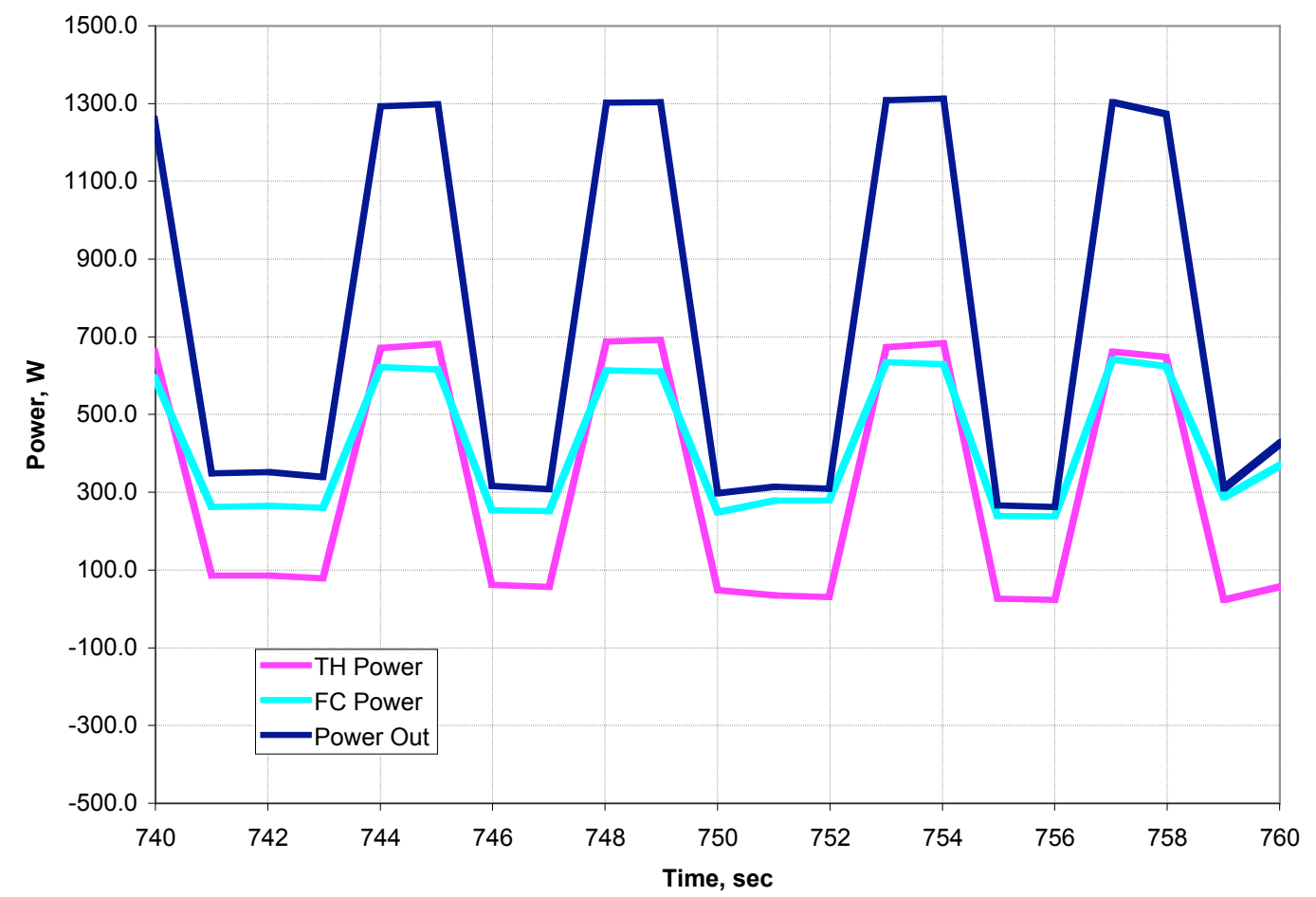

Fig. 128. Experimental data of fuel cell and transient handler.

(Working together to deliver $1.3 \mathrm{~kW}$ of pulsed power.) 
Figure 129 shows the power electronics module that is responsible for regulating the output of the energy storage system and controlling the state of charge for the transient handler. Figure 130 is a picture of the system controller. Figure 131 shows the hydrogen generation module - it consists of a catalytic reactor, developed at ORNL, a spent solution chamber and a fresh solution chamber. A small pump is used to pump fresh $\mathrm{NaBH}_{4}$ solution through the catalytic reactor. The hydrogen produced is cooled off to stack temperature and fed to the fuel cell. Whenever the hydrogen pressure drops below a set value, new solution is pumped into the catalytic reactor. The spent solution accumulates in the reservoir that is also used as a phase separator and prevents liquid from entering the fuel cell stack. A fine mesh filter is also used to make sure that the produced hydrogen does not carry any small liquid particles into the fuel cell stack. The air needed for fuel cell operation is provided by an off-the-shelf compressor. Its speed is controlled by the BSC and is proportional to the fuel cell stack current. The fuel cell stack temperature is also under control of the BSC - in four stages - the fan speed can be set at zero, low, medium and high in order to conserve energy.

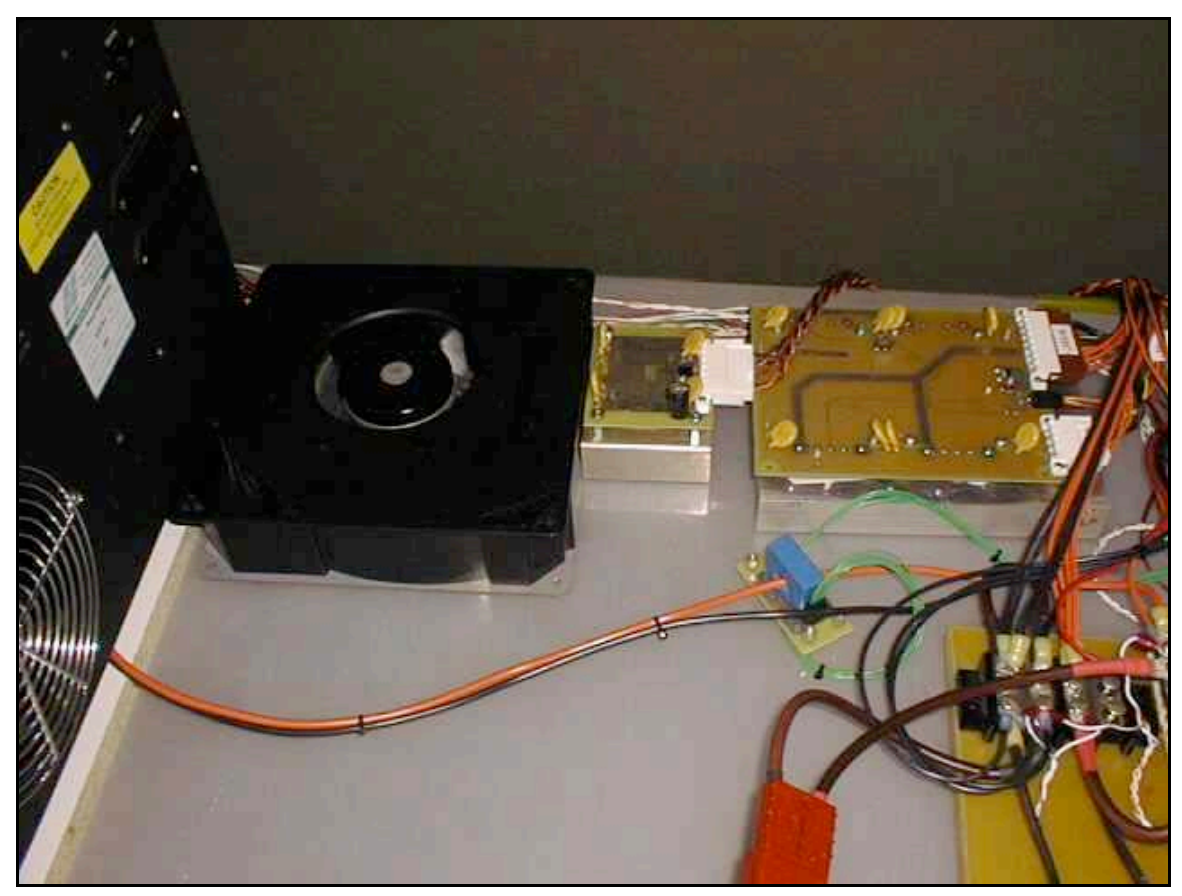

Fig. 129. Power electronics module. 


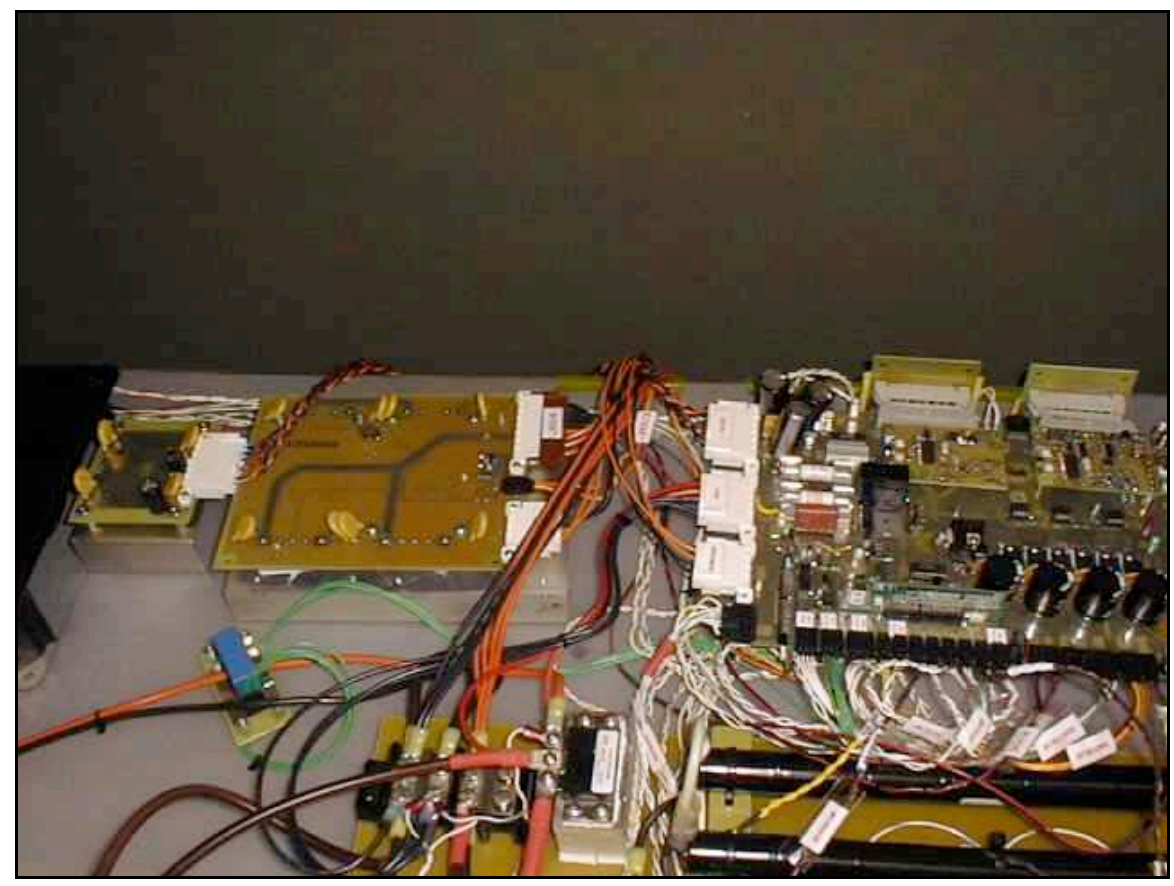

Fig. 130. System controller module.

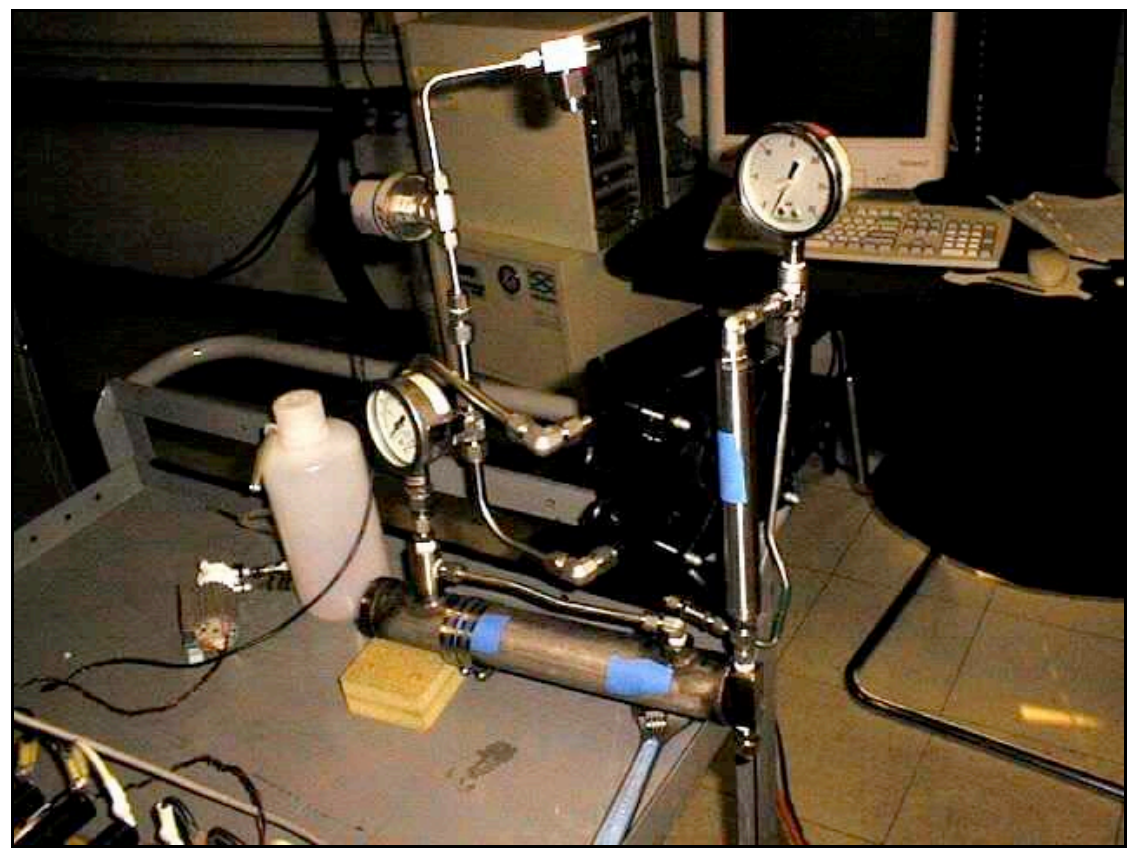

Fig. 131. Hydrogen generation module.

Figure 132 shows the fuel cell stack module with a data acquisition system that measures every cell voltage in the stack. The system consists of five FC-SG modules, of 12 channels each. The modules are connected to the system controller via a fast serial bus. The data acquisition system was very useful when tuning of the system was performed but may not be needed when it is fully developed. Figure 133 shows the transient handler module. It is also instrumented with voltage and temperature measurement devices so that it's state-of-charge and health can be tracked. The 
state of charge of the transient handler is monitored and controlled by the BSC with an elaborate algorithm that takes into account temperature, Ah depletion/addition, and average cell voltage. As can be seen in Fig. 127, all the components can fit on a small cart-and can be easily scaled-depending on the power needs and energy storage requirement imposed on the system.

All experiments with the current system were performed using a diluted solution of less than $17 \mathrm{wt} \%$ of $\mathrm{NaBH}_{4}$ in water.

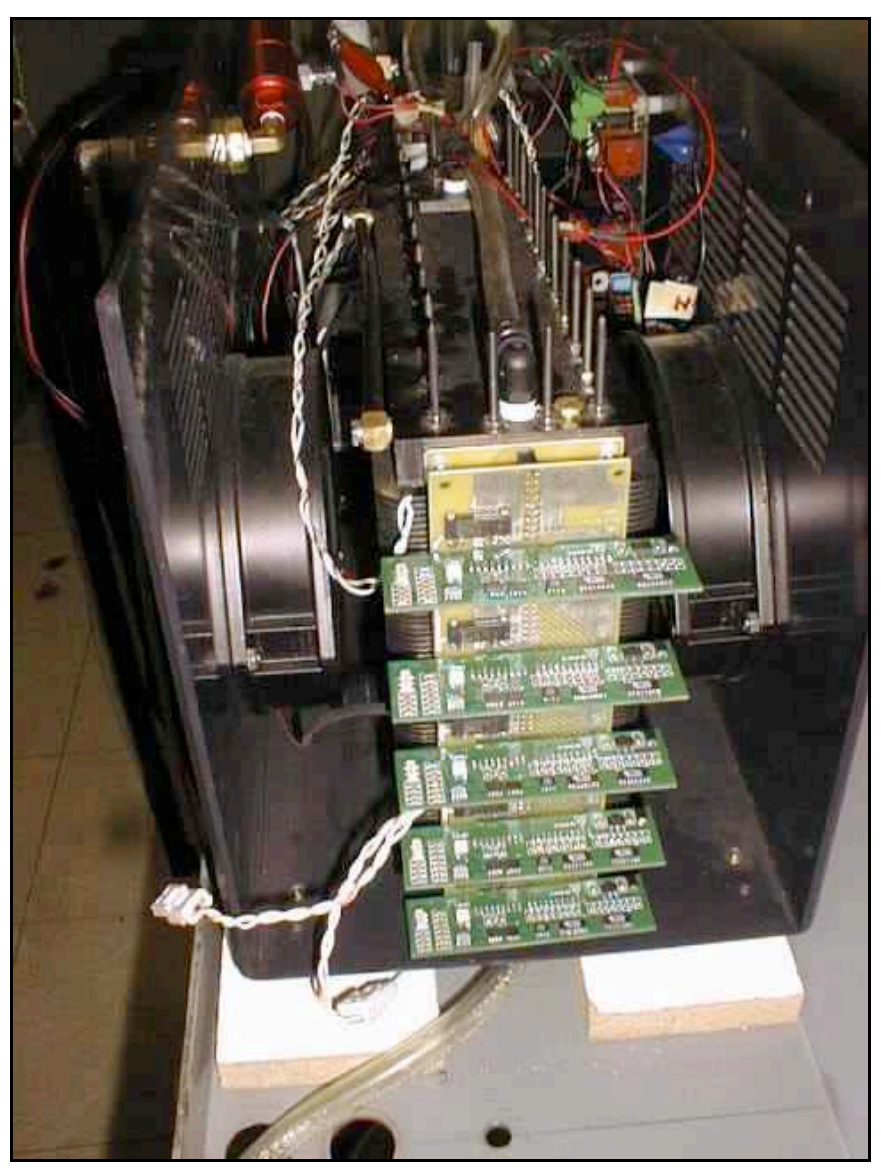

Fig. 132. Fuel cell module. 


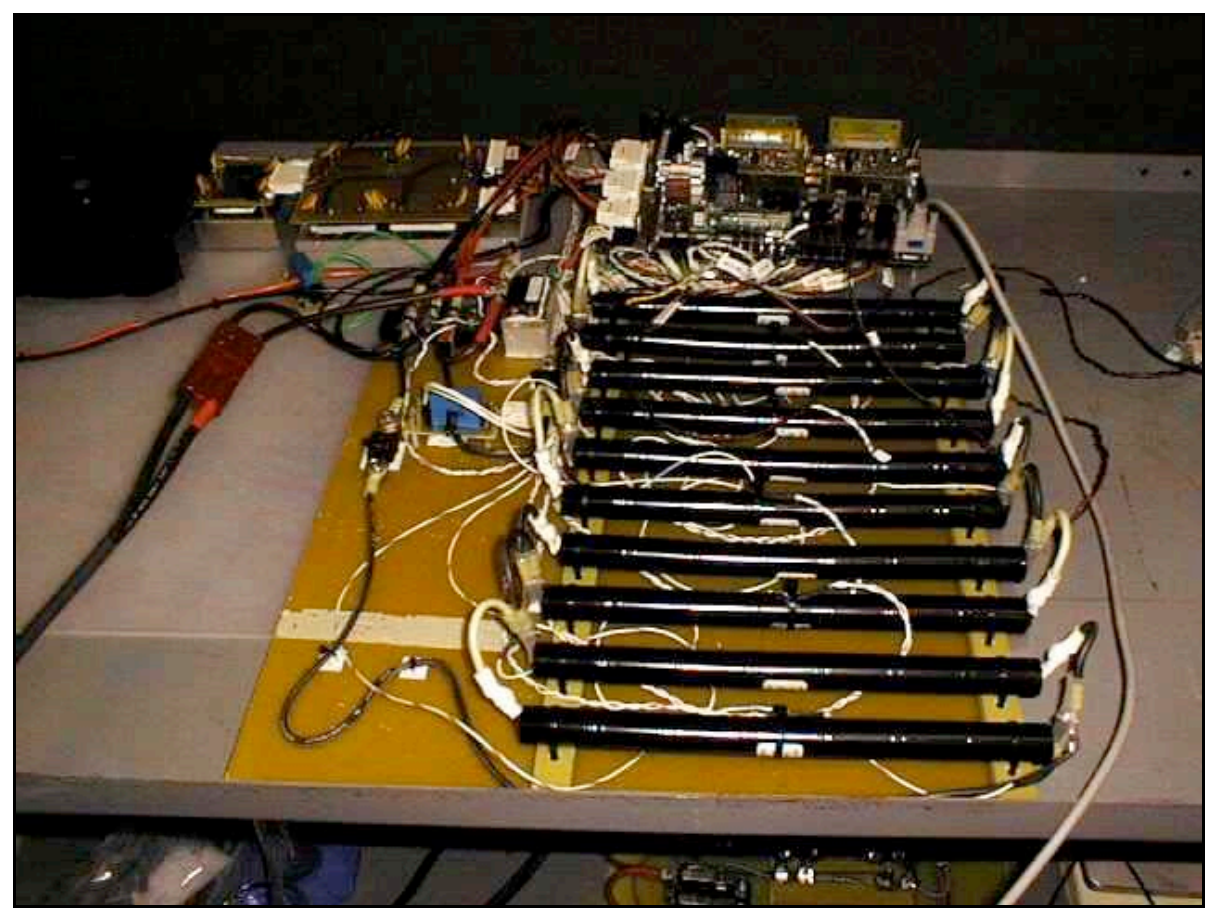

Fig. 133. Transient handler module.

\section{CONCLUSIONS AND RECOMMENDATIONS}

\section{General Findings}

The project was very successful in showing that a fuel cell hybrid power supply can be a viable option for supplying the power and energy needs of an exoskeleton. In addition, the project demonstrated that the hybrid power supply system is intrinsically safe, and can be operated from hydrogen created on demand by a package of sodium borohydride.

What worked and what did not - the system met all requirements of the project. There could be more tuning of the algorithm for power sharing and keeping the fuel cell operating at close to full power whenever needed in order to extend the availability of the transient handler. We had some difficulties with the stability of the sodium borohydride solution and the pump used to feed the catalytic reactor. It is desirable to reuse the water produced in the fuel cell and react it with dry sodium borohydride in order to increase the specific energy of the system.

Expected results and surprises - it is important to note that the fuel cell could successfully deliver power in a pulsed mode, even when operated with air compressor and hydrogen generated from chemical hydride.

\section{General Recommendations}

Recommendations for future work - the system described in this report is a brass board system built with mostly off-the-shelf components. The next task would be to find the lightest, most efficient components. There is significant room for size reduction, increased efficiency and simplified operation. The system can be packaged with different form factors. 
In order to increase the specific energy of the system, a fuel cell product water recovery system should be incorporated in the design, with the capability to store the sodium borohydride in a dry form and mix it with the product water from the fuel cell, as needed.

Applicability to other projects/uses - The hybrid fuel cell system built for this project has potential for many other uses. For example, with slight modifications the control system could be a component for many other projects with similar architecture but in a significantly wide range of power ratings - up to $100 \mathrm{~kW}$ systems. 
APPENDIX G

FEASIBILITY STUDY - ACTUATOR COMPARISON 
This appendix is an excerpt from an ORNL exoskeleton feasibility study conducted for DARPA (Jansen et al., 2000). This appendix provides some of the background and basis for the design approach taken and is included here for completeness and the convenience of the reader.

The purpose of this section is to compare various types of actuator technologies subject to their relevance to the exoskeleton project. This section is not meant to be a primer about actuators but a comparison of potential relevant technologies. A designer must make decisions based on critical design parameters such as (1) power/mass, (2) power/volume, (3) stress, (4) strain, (5) steady-state efficiency, (6) power expended during load holding, (7) bandwidth or tracking performance, (8) auxiliary transmission system, (9) auxiliary power modulation equipment, and (10) ease of controllability. Depending on the type of actuator, some of the critical design parameters will not be applicable or not available due to design specificity. Actuator technologies will be broken down into two major groups: conventional and non-conventional. Conventional actuators are electric motors, hydraulic, and pneumatic. The non-conventional actuators are the piezoelectric and magnetorestrictive. There will be a brief discussion of the shape memory alloy and polymers. While others have examined these types of actuators in the past, their relevance to the exoskeleton project will be the main focus on this section. Before comparisons can be made, a brief description of the demands that will be imposed on the actuators of an exoskeleton will be given.

\section{EXOSKELETON ACTUATOR DEMANDS}

To understand the magnitude of torques and power required for exoskeleton actuators, loads for a human will be scaled to account for the higher weight of a loaded exoskeleton system. The loads are based on the assumption of a $150 \mathrm{lb}$ individual with a $150 \mathrm{lb}$ load to represent a $300 \mathrm{lb}$ exoskeleton system (exoskeleton plus load). Only two joints, the hip and knee joints, will be considered to understand the load and power required for an exoskeleton actuator. Studies at ORNL, which looked at a variety of poses, showed a kneeling pose yielded the largest loads of $1,350 \mathrm{in}-\mathrm{lb}(153 \mathrm{~N}-\mathrm{m})$ for hip joint and $6300 \mathrm{in}-\mathrm{lb}(712 \mathrm{~N}-\mathrm{m})$ for knee joint. Examination of a simple knee-bend-type of movement, where the human and load are moving vertically up and down with respect to gravity with 17.5 in of motion in $1 \mathrm{~s}$, shows power levels for the knee joint can approach $600 \mathrm{~W}$ and for the hip joint around $120 \mathrm{~W}$. Peak knee-joint velocities can approach almost $200 \%$ and for the hip peak velocities around $100 \% \mathrm{~s}$ are possible. For walking and running, peak joint velocities can go up by a factor of three times these numbers.

Based on human-motion profiles for simple ballistic-type of walking (McMahon, 1984), a periodic gait pattern of around $2 \mathrm{~Hz}$ can easily be seen, and even higher numbers are possible for other types of motions (e.g., running). Power requirements for simple ballistic walking will be significantly lower than for a deep-knee-bend-type of movement; however, the actuator would have to be rated for the worst-case scenario.

To evaluate different types of actuators, actuators around the 500 to $1000 \mathrm{~W}$ levels will be discussed with thousands of in-lb of torque capability and with the closed-loop bandwidth to accurately track a $2 \mathrm{~Hz}$ gait. 


\section{CONVENTIONAL ACTUATORS}

Conventional actuators are those that are common for robotic manipulators. Basically, there are three major types: electromagnetic (electric motors), pneumatic, and hydraulic. For the electromagnetic category, only brushless dc motors will be examined since they are the closest match to the intent of this project. Reluctance-based motors such as those from Nippon Seiko (NSK) called Motornetics Megatorque ${ }^{\mathrm{TM}}$ motors will not be examined because, while they have a somewhat higher torque to mass ratio than do standard brushless dc motors, their overall larger diameters and associated power electronics puts them at a disadvantage pertaining to packaging. Pneumatic and hydraulic actuators based on classical servovalve control will be examined and will be compared to each other.

\section{Electromagnetic Actuators (Brushless Motors)}

About 50 motors from Inland Motors, Inc., were examined. All were high-end frameless, brushless motors. A plot of the continuous power versus mass is shown in Fig. 134. For electric motors (specifically those around $0.5 \mathrm{~kW}$ to $1 \mathrm{~kW}$ level) the power-to-mass ratio can be bounded by a line, which has a slope of roughly $500 \mathrm{~W} / \mathrm{kg}$. The continuous torque out of an electric motor per unit total motor volume is shown in Fig. 135.

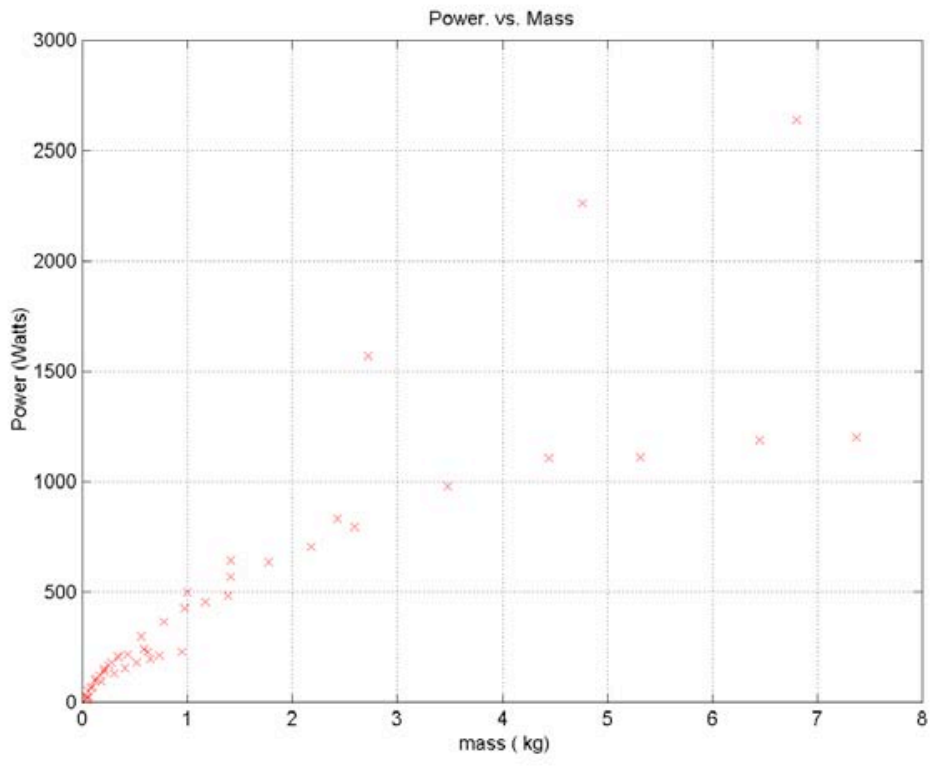

Fig. 134. Power vs mass for frameless brushless dc motors. 


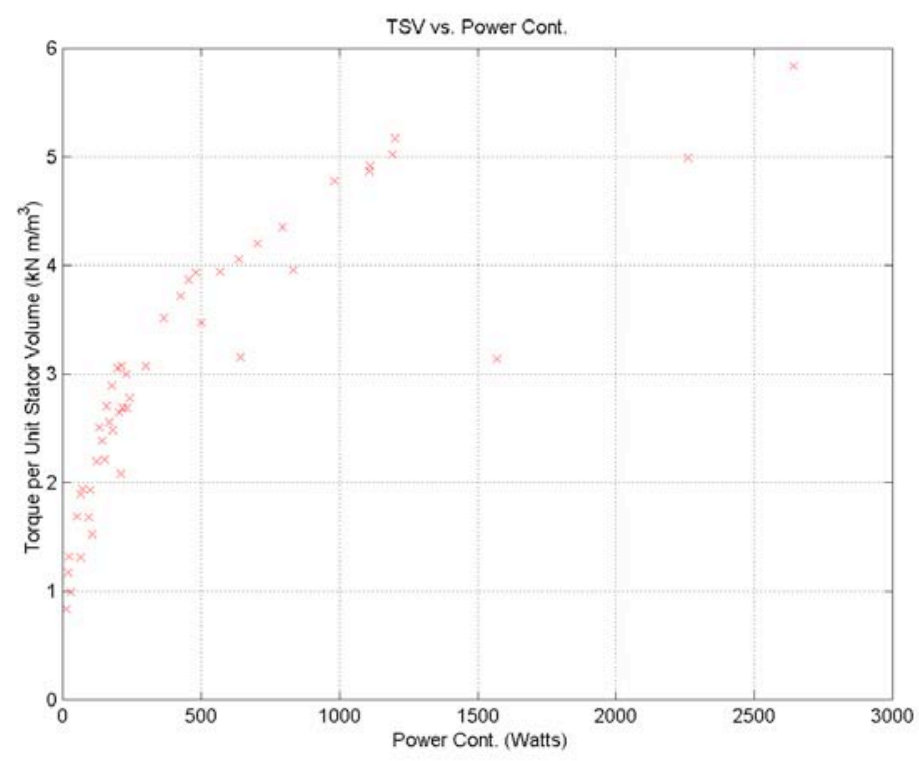

Fig. 135. Torque per unit motor volume vs continuous power.

For 500- to 1000-W motors, the continuous torque per unit motor volume varies from 4 to $5 \mathrm{KN} / \mathrm{m}^{3}$. Figure 136 shows the continuous torque per mass for the same power range, which is under $3 \mathrm{Nm} / \mathrm{kg}$. The continuous torque per unit volume and continuous torque per unit mass ratios will increase if the thermal operating range of the motors can be exceeded (e.g., air cooling, special higher temperature rated wires). Some researchers cite numbers twice as high as the ones given (Hollerbach, 1992), which can be obtained by the so-called megatorque motors, which are manufactured by NSK; however, as previously mentioned, the package volume of these motors are probably unsuitable for an exoskeleton.

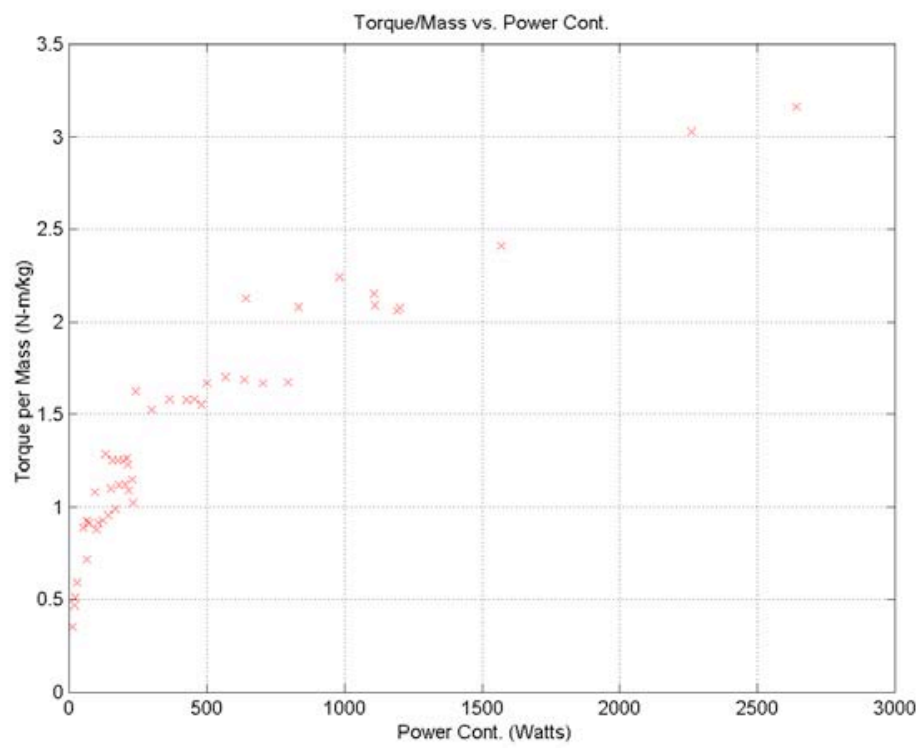

Fig. 136. Torque per mass vs power continuous. 
Upper values for the shear stress over the rotor surface of an electric motor have been reported to be under 5 psi (higher values are possible if a cooling system is added). These low values of shear stress are not subject to significant improvements and are currently limited by heat transfer and the magnetic saturation limits of supporting ferrous structure (Miller, 1989). Steady-state electromechanical efficiency is around 85 to $95 \%$. The power required for holding rated torque at stalled conditions can range from 5 to $10 \%$ of the power rating of the motor. Basically, this means that while no mechanical power is being expended, significant power is required to merely hold the load. Mechanical brakes are typically not an option for a high-performance servo system.

Based on the $600 \mathrm{~W}$ required for the knee-joint actuator and the $120 \mathrm{~W}$ for the hip joint, and using a $500 \mathrm{~W} / \mathrm{kg}$ value for actuator mass sizing, a $1.2 \mathrm{~kg}$ actuator for the knee and $0.24 \mathrm{~kg}$ actuator for the hip would be required. The equivalent torque rating for these actuators, based on the $3 \mathrm{Nm} / \mathrm{kg}$ torque sizing value, would be $3.6 \mathrm{~N}$-m for the knee joint actuator and $0.72 \mathrm{~N}-\mathrm{m}$ for the hip joint actuator. Since we need a $712 \mathrm{~N}-\mathrm{m}$ for the knee joint, a transmission system (e.g., gearbox) with about a 200:1 ratio is needed for the knee joint. Likewise, for the hip joint we need $153 \mathrm{~N}-\mathrm{m}$, and a transmission system again of about a 200:1 ratio will be required. Steady-state transmission efficiencies are typically 80 to $90 \%$, which means that the motors would have to be 10 to $20 \%$ larger from a power perspective. Depending on the type of transmission system, such as a harmonic to a planetary type of gearhead, the transmission system can increase the overall package volume by $20 \%-50 \%$. For simplicity, the impact of the actuators and transmission system on the overall weight of the exoskeleton, while not insignificant, has been ignored in terms of the joint velocity.

From Fig. 137, we see that the maximum unloaded speed of an electric motor is under 75 revolutions/s. When divided by the 200:1 gear ratio, the maximum unloaded angular velocity is $123 \%$. Since running will require significantly higher peak joint velocities, a significantly larger motor size will be required by roughly a factor of 2 (i.e., the power density will decrease by a factor of 2) to achieve adequate joint velocities. In summary, a transmission system is needed for an electric system, and it can significantly impact the overall package volume and efficiency. To track peak joint velocities, the motor size will have to be increased significantly because of the high gear ratios required.

The volume and mass of the power electronics associated with an electric motor vary greatly depending on the power source (i.e., dc or ac) and how close the voltage of the supply is to the voltage needed by the motor. Typically, on industrial robotic manipulator systems (where weight is not a major concern), the power electronics can easily exceed the weight and volume of all the actuators. Power-to-volume densities are typically in the 2- to $40-\mathrm{W} / \mathrm{in}^{3}$ range (as compared to around $25 \mathrm{~W} /$ in $^{3}$ for electric motors). By judicial design, the volume and mass of the associated power electronics can be significantly reduced, but not without a significant design effort. 


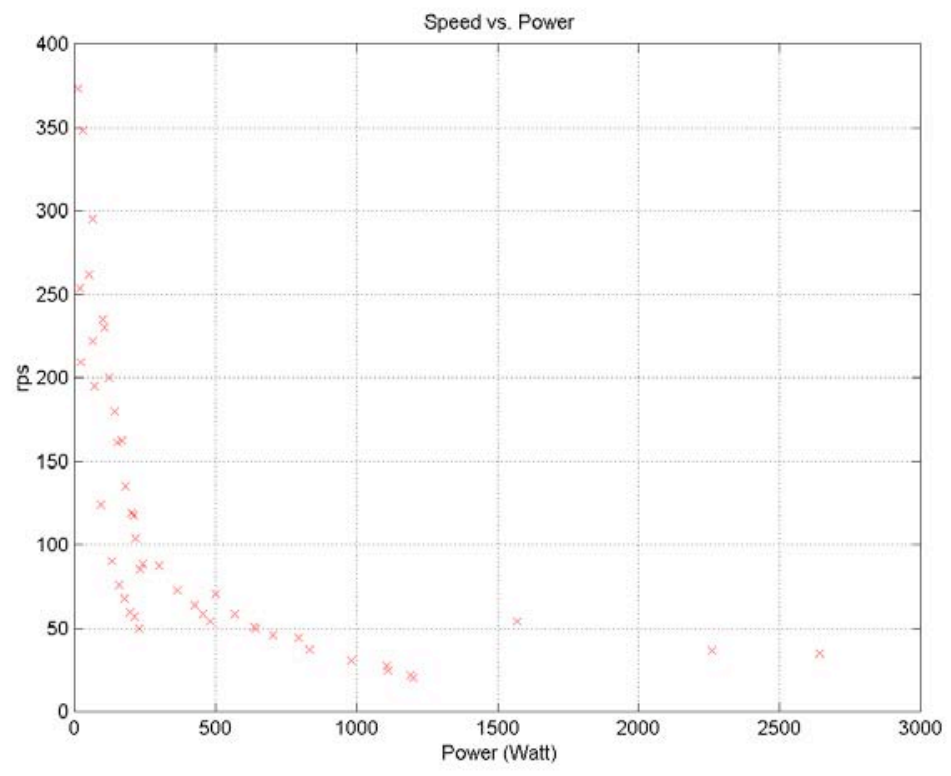

Fig. 137. Speed (revolutions/s) vs continuous power.

\section{Hydraulic Actuators}

Hydraulic actuators are fundamentally different than electric motors in that the amount of flow into a hydraulic actuator is limited by the maximum amount of flow possible from the pumping source and any flow restrictions caused by flow control elements such as servo valves. Thermal limitations associated with electric motors do not apply to hydraulic actuators since the hydraulic fluid cools and lubricates the system. Material restrictions are based only on pressure limits. As a rule of thumb, the power to mass ratio is about five times that of an electric motor and the power to volume ratio is about ten times that of an electric motor for many applications. Again, these numbers could be higher or lower depending on the particular application. The pressure levels are from 3,000 to 5,000 psi (or 21 to $35 \mathrm{MPa}$ ), with 3,000 psi being an industrial standard. Maximum strain levels correspond to the stroke length over the total actuator length, which can range from 0.5 to 0.8 for most actuators. For rotary actuators, the maximum strain levels do not apply. Unlike the electric motor, the force or torque that can be produced can be easily matched to the application simply by changing the effective area for a linear actuator or the effective displacement for a rotary actuator. Therefore, a transmission system is typically not required. Only the friction at the seals and leakage affects limit the steady-state mechanical efficiency. For linear actuators, high, steady-state mechanical efficiencies are possible and are in the upper $90 \%$ range. Rotary actuators range from 80 to $90 \%$. For load-holding applications, zero power is expended for linear actuators with asymmetrical cylinders and a small amount of power is expended in the 1 to $10 \%$ range because of leakage for symmetrical cylinders and rotary actuators.

The bandwidth of a hydraulic actuator can be derived based on a few simplifying assumptions; (1) only a symmetrical cylinder design will be assumed; (2) fluid volume outside of the cylinder will be ignored; and (3) half of the system pressure is used to hold the load against gravity. In addition, the fluid bulk modulus will be assumed to be at 100,000 psi (or $689 \mathrm{MPa}$ ) and the 
system pressure is set at $3000 \mathrm{psi}(21 \mathrm{MPa})$. Starting with the natural frequency, $\mathrm{f}_{\mathrm{o}}$ (in $\mathrm{Hz}$ ), the derivation of a hydraulic cylinder is (Viersma, 1980)

$$
\mathrm{f}_{\mathrm{o}} \geq \frac{1}{\square} \sqrt{\frac{\mathrm{A} \square}{\mathrm{MS}}}=\frac{1}{\square} \sqrt{\frac{\mathrm{A} \square \mathrm{g}}{\mathrm{MgS}}}
$$

or

$$
\mathrm{f}_{\mathrm{o}} \geq \frac{1}{\square} \sqrt{\frac{\square \mathrm{g}}{\frac{\mathrm{Mg}}{\mathrm{A}} \mathrm{S}}}=\frac{\sqrt{2}}{\square} \sqrt{\frac{\square \mathrm{g}}{\mathrm{P}_{\mathrm{s}} \mathrm{S}}}
$$

which can be further simplified to

$$
\mathrm{f}_{\mathrm{o}} \sqrt{\mathrm{S}} \geq \frac{\sqrt{2}}{\square} \sqrt{\frac{\square \mathrm{g}}{\mathrm{P}_{\mathrm{s}}}}=8.1
$$

where

$$
\begin{aligned}
& \mathrm{M}=\text { load mass }(\mathrm{kg}) \\
& \mathrm{A}=\text { effective stroke area }\left(\mathrm{m}^{2}\right), \\
& \mathrm{P}_{\mathrm{s}}=\operatorname{supply} \text { pressure }(3000 \mathrm{psi}), \\
& \mathrm{S}=\operatorname{stroke} \text { length }(\mathrm{m}), \\
& \mathrm{g}=\text { gravity constant }=9.807 \mathrm{~m} / \mathrm{s}^{2}, \text { and } \\
& \square=\text { fluid bulk modulus constant }(100,000 \mathrm{psi}) .
\end{aligned}
$$

As an example for a stroke length of 6 in $(0.1524 \mathrm{~m})$, the natural frequency would be above $21 \mathrm{~Hz}$. Depending on the type of controller (i.e., position or pressure acceleration and type of feedback), the closed-loop bandwidth could be as low as 0.15 [for positional feedback, (Merritt, 1967)] to 0.5 [for acceleration feedback, (Viersma, 1980)] of the natural frequency value with 0.15 typical for industrial applications. For this example, the closed-loop bandwidth would typically be greater than $3 \mathrm{~Hz}$.

Control of a hydraulic actuator for high-precision applications is typically through a servo valve. The bandwidth for most high performance servo valves that would be applicable to the exoskeleton project would be typically from 50 to $100 \mathrm{~Hz}$ (see Moog's model 30 valves at www.moog.com). Losses associated with a servo valve could be as high as about 1/3 of the maximum power to the hydraulic actuator since most servo valves are rated for a 1,000-psi drop across their orifices. Reliability of a hydraulic actuator is mainly limited by seal and/or gland wear and impact loads. Servo valves associated with a hydraulic actuator are more likely to fail. However, with suitable control of the overall filtration levels of hydraulic fluid, servo valves can have a long service life (exceeding over one quarter million hours mean time before failure for valves such as the deflector jet type) as noted by their application in the commercial aircraft industry. 
The power electronics associated with the hydraulic servo valve and actuator can be inconsequential. For a two-stage servo valve, the drive current for the torque motor is typically well within the linear operational amplifier range (e.g., the Moog's model 30 is typically rated around $16 \mathrm{~mA}$ ). This again adds to the advantage of hydraulic actuators as compared to electric motor actuators.

One final point associated with hydraulic servo systems which needs to be mentioned, is the noise problem associated with the pumping system. Hydraulic pumps are typically noisy at fairly low frequency, thus making sound proofing at reasonable packaging volumes difficult because of the large acoustical wavelengths.

\section{Pneumatic Actuators}

Pneumatic actuators are very similar to the hydraulic actuators in many respects. However, a few exceptions are especially noteworthy. Air is typically a poor lubricating and cooling medium, as compared to a hydraulic fluid medium. Losses are higher because of fluid leakage through seals. Furthermore, air is significantly more compressible than a hydraulic fluid medium. Compressibility of air will significantly lower the natural frequency of a pneumatic control system. Significant energy can be stored in this medium, which can be used to one's advantage, such as mitigating impact loads, or it can be a safety problem because of the possible burst hazard it presents. In comparison, hydraulic fluid has no significant energy storage capability. Also, as a practical limitation, the supply pressure for a pneumatic storage device is limited to 100 to $150 \mathrm{psi}$ as compared to 3,000 to 5,000 psi, for a hydraulic system. Power to weight and power to volume for pneumatics can vary depending on the type and application. For elastic-type pneumatic actuators, such as the McKibben muscle (Chou, 1996), these ratios can rival hydraulic actuators. For hard-shell-type pneumatic actuators, these ratios are closer to electric actuators; however, the pneumatic actuators are only limited by the mass flow from the pump and any flow restrictors such as a servo valve.

While at first glance pneumatic actuators hold many advantages such as (1) high power to weight and power to volume (for elastic type pneumatic actuators), (2) good impact load mitigation, and (3) energy storage capability; there are some important limitations. While losses are typically higher than hydraulic and safety is always a concern, the main barrier for pneumatic types of actuators is the bandwidth caused by the compressibility of the air. Based on similar assumptions made for a hydraulic actuator and starting with the basic natural frequency formulation of a pneumatic cylinder (Andersen, 1967)

$$
\mathrm{f}_{\mathrm{o}} \geq \frac{1}{\square} \sqrt{\frac{\mathrm{k}_{\mathrm{p}}}{\mathrm{M}}}=\frac{1}{\square} \sqrt{\frac{4 \mathrm{nP}_{\mathrm{s}} \mathrm{A}_{\mathrm{p}}^{2} \mathrm{~g}}{\mathrm{MgV_{ \textrm {t } }}}}
$$

or

$$
\mathrm{f}_{\mathrm{o}} \geq \frac{1}{\square} \sqrt{\frac{\mathrm{n \textrm {P } _ { \mathrm { s } } g}}{\frac{\mathrm{Mg} \mathrm{V}_{\mathrm{t}}}{\mathrm{A}_{\mathrm{p}} \mathrm{A}_{\mathrm{p}}}}}=\frac{1}{\square} \sqrt{\frac{\mathrm{ng}}{\mathrm{S} \frac{\mathrm{Mg}}{\mathrm{A}_{\mathrm{p}}} / \mathrm{P}_{\mathrm{s}}}}=\frac{\sqrt{2}}{\square} \sqrt{\frac{\mathrm{ng}}{\mathrm{S}}}
$$


which can be further simplified to

$$
\mathrm{f}_{\mathrm{o}} \sqrt{\mathrm{S}} \geq \frac{\sqrt{2}}{\square} \sqrt{\mathrm{ng}}=1.4 \quad \text { to } \quad 1.7
$$

where

$\mathrm{n}=$ polytropic exponent which can vary from 1 to 1.4 (ratios of specific heat for air),

$\mathrm{k}_{\mathrm{p}}=$ effective stiffness of gas in cylinder, and

$\mathrm{A}_{\mathrm{p}}=$ effective area of pneumatic cylinder.

Comparison of the natural frequency of hydraulic and pneumatic actuators by means of Eqs. (39) and (40), clearly shows that for a given stroke length the natural frequency of hydraulics is 5 to 6 times higher than pneumatic actuators. Regardless of the type of feedback scheme, since whatever can be applied to a pneumatic servo can be applied to a hydraulic servo, the closedloop bandwidth of a hydraulic system will be 5 to 6 times higher than a pneumatic servo-system for the same stroke length. For the example in the previous section, the hydraulic closed-loop bandwidth was calculated to be greater than $3 \mathrm{~Hz}$, while for pneumatics, it would be anticipated to be greater than $0.5 \mathrm{~Hz}$. Closed loop tracking at $2 \mathrm{~Hz}$, for this example, can only be achieved with hydraulic actuators.

\section{NON-CONVENTIONAL ACTUATORS}

Non-conventional actuators are those that are typically described as associated with smart materials, such as piezoelectric, magnetostrictive, shaped memory alloys, and electroactive polymers. While there are others (such as those associated with magnetorheological and electrorheological fluids, and electrostrictive materials), only a few of these materials are seriously being considered as viable candidate for the exoskeleton project. In the future and after more research, these other candidates could play an important role in actuator applications.

\section{Piezoelectric Actuators}

Large forces and small displacements occur in a piezoelectric crystal when an electric field is applied. Compressive stress levels can be as high as $35 \mathrm{MPa}(5,000 \mathrm{psi})$, whereas the tensile stress levels can be only 5 to $10 \%$ of the compressive stress level. Typically, a mechanical force bias is required to avoid the tensile stress limits. Displacements are typically very small for piezoelectric materials. Currently, PZT ceramics are the materials of choice for piezoelectric ceramic actuators. These materials have piezoelectric coefficients $\mathrm{d}_{33}$, around 200 to $750 \mathrm{pC} / \mathrm{N}$, and strain levels from 0.05 to $0.1 \%$. However, there is a new piezoelectric material that has the potential to change future piezoelectric actuators. This new material, a single crystal form of relaxor-based ferroelectric materials (PZN-PT), has been observed to have piezoelectric coefficients $\mathrm{d}_{33}$, around $2,000 \mathrm{pC} / \mathrm{N}$, and strain levels from 0.5 to $1.0 \%$. While these materials

have been known for several years, their potential for high performance actuators has recently been recognized (Park, 1997). Applications that require high forces and small displacements, such as those in acoustic applications are immediate application areas. However, ferroelectric 
crystals experience a hysteresis effect, which make them difficult to control for high-precision applications.

One difficulty of using piezoelectric material pertains to converting small displacements to large motion (i.e., the transmission problem). Various ideas include the "inch-worm," where microstepping action through rapid lock-and-move sort of motion are used to create a large linear motion. Another concept is that of a piezoelectric hydraulic pump (Nasser, 2000), where small quantities of fluid moves at very high frequencies. The accumulation of small drops of fluid at a high rate adds up to a large flow rate. Both of these concepts are based on the idea of the cyclic motion of the piezoelectric material. A simplified electromechanical model is shown in Fig. 138. The left-side ports are the electrical ports where a voltage is applied and current is injected into the crystal. The right-side port is where force represents the equivalent voltage and velocity represents the current. A mechanical load, $\mathrm{Z}_{\mathrm{m}}$, is shown attached to the mechanical port.

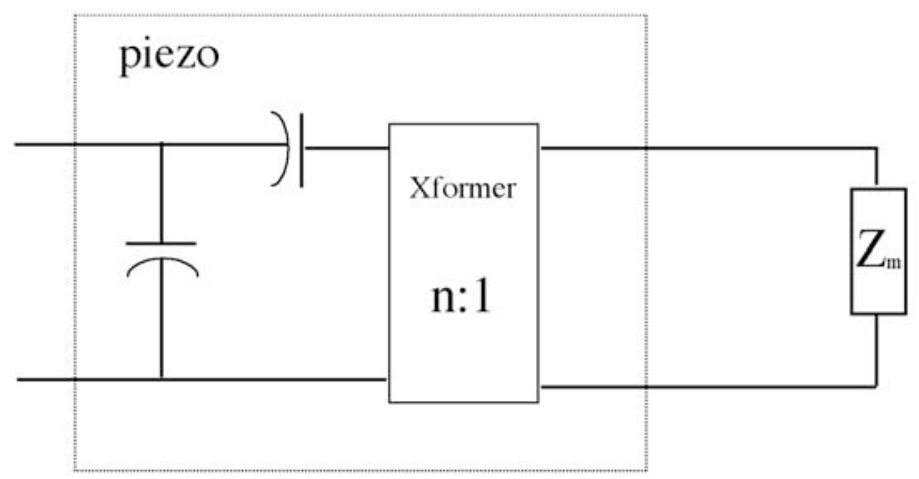

Fig. 138. Electromechanical model of piezoelectric actuator with mechanical load.

If the mechanical load can be represented as a purely resistive load that has been optimized to achieve maximum power transfer at a given frequency, $\square$, then it can be shown that the power per volume of the piezoelectric actuator can be approximated to

$$
\frac{\text { power }}{\text { volume }}=\frac{\square_{1}}{=2} \square_{33}^{\mathrm{T}} \mathrm{E}^{2} \mathrm{Z}^{2} \mathrm{a}
$$

where

$$
\begin{aligned}
& \mathrm{E}=\text { electric field, typically under } 10^{6} \mathrm{~V} / \mathrm{m}, \\
& \square_{33}=\text { electric permittivity, and } \\
& \mathrm{k}^{2}=\text { electrical to mechanical conversion constant }(0.75 \text { for PZN). }
\end{aligned}
$$

The term in parenthesis is the electrical energy density stored in the piezoelectric material, and for modest electric fields, this term could have values of $0.02 \mathrm{~J} / \mathrm{cm}^{3}$ (or $2.7 \mathrm{~J} / \mathrm{kg}$ for a typical density value of $8,200 \mathrm{~kg} / \mathrm{m}^{3}$ ) for the single-crystal piezoelectric material PZN. Larger energystorage terms have been reported in the literature, but because of fatigue life limitations (remember that because of the transmission problem, billions of cycles will be required from this material), this reduced value is more reasonable. To obtain over $2,500 \mathrm{~W} / \mathrm{kg}$ (which is a factor of 5 times that of an electric motor and would therefore rival that of hydraulic), the cyclic 
frequency would have to be over $200 \mathrm{~Hz}$. Because of the nonlinear nature of the load expected for the "inch worm" or the piezoelectric pump, a much higher frequency (maybe around $1 \mathrm{kHz}$ ) would be required. The steady-state efficiency of running the actuator around $1 \mathrm{kHz}$ would be approximately $85 \%$. The load-holding capacity of the piezoelectric material is excellent - no power is wasted.

The final major challenge associated with piezoelectric actuator is the associated power electronics to control it. Typical piezoelectric actuators are driven by high-voltage power sources of around 400 to $1,000 \mathrm{~V}$. The size of the drive electronics frequently exceeds the overall packaging volume of the actuator by one order of magnitude if not larger. The basic problem of power transfer can be seen in Fig.137. How one can transmit charge readily to the mechanical load without wasting the energy stored in the two capacitors is the salient design problem and is still under research (Newton, 1996).

\section{Magnetostrictive Actuators}

Magnetostrictive actuators are very similar to piezoelectric actuators. Applying a magnetic field creates high forces and small displacements. The magnetic domains in the material rotate causing significant dimensional change in the material. Currently, Terfenol-D is the magnetostrictive material in common use. Large strains are typically reported, but in practice values under $0.2 \%$ are commonly observed. Stress values, while reported to be higher than piezoelectric materials, are commonly below commercial PZT values when a direct comparison is done (see www.etrema-usa.com for Terfenol-D actuators and www.physikinstrumente.com/pztactuators for the PZT actuators). All of the issues associated with piezoelectric actuators also apply to magnetostrictive actuators. The simplified electromechanical model of the magnetostrictive actuator is shown in Fig. 139, where the left port is the electrical port with current and voltages as inputs and the output port is the mechanical port with velocity and force. The load-holding capability of the material is limited by the $i^{2} R$ losses associated with generating a magnetic field and is similar to electric motors with a range of 4 to $8 \%$ of the continuous power rating for maximum load. Overall efficiency numbers are hard to obtain, but due to the highly nonlinear nature of the hysteresis of the magnetic material and the $\mathrm{i}^{2} \mathrm{R}$ losses associated with the electrical coils, efficiencies should be less than those for PZT materials.

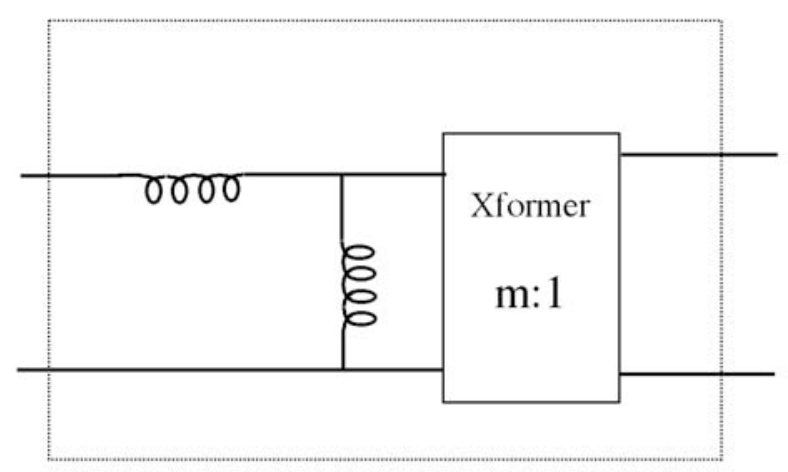

Fig. 139. Magnetostrictive electromechanical model. 
The issues associated with the power electronics also apply to the magnetostrictive materials. The basic problem of power transfer can be seen in Fig. 139. How one can transmit current readily to the mechanical load without wasting the energy stored in the two inductors is the salient design problem and is still under research (Kellogg, 1996).

\section{Shaped Memory Alloys (SMAs) and Electroactive Polymers (EAPs)}

Shared Memory Alloys (SMAs) (NiTi), while having impressive compressive stresses of $200 \mathrm{MPa}$ (i.e., they can pull but not push), have overall energy efficiencies under 3\%. Without large amounts of cooling, overall bandwidth will be below $1 \mathrm{~Hz}$. SMA are not serious contenders for the exoskeleton application unless the overall efficiencies and bandwidth issues can be seriously extended.

Electroactive Polymers (EAPs) are an exciting new material that has many anthropomorphic characteristics similar to muscle. In the majority of EAP actuators, the actuation mechanism is based on ionic species movement in a polymer network (Wax, 1999). EAP actuators can be characterized as gels; ionic polymer metal composites; perfluorinated, ion-exchanged, membrane platinum; or conductive polymers. EAP actuators hold tremendous potential especially for microactuation applications. For macroactuation applications, such as for the exoskeleton project, speed of response (i.e., bandwidth) and higher levels of electrical-to-mechanical power conversion ratios are needed. Currently, macro EAP actuators fall well below electric motor power and force (or torque) densities (Wax, 1999).

\section{ACTUATOR RECOMMENDATION}

Of the conventional actuators (Table 24), it appears that hydraulics is the best candidate for an exoskeleton application. The power-to-weight and power-to-volume ratios of hydraulics typically exceed the ratios for electromagnetic actuation by factors of 10 and 5 respectively. The closed-loop bandwidth of pneumatics is typically 5 to 6 times less than that for hydraulics, thus limiting the ability to track at $2 \mathrm{~Hz}$. Based on packaging and the ability to track the human, hydraulics is the preferred conventional actuator.

However, there are issues concerning the use of hydraulics, which must be taken into account. First, the power loss at the servo valves associated with flow control is significant and has a serious impact on the overall power supply sizing. Second, noise is a serious problem associated with hydraulics that can be difficult to mitigate due to the large acoustical wavelengths.

While none of the non-conventional materials have reached the level of maturity achieved with hydraulics, both the piezoelectric and the magnetostrictive materials hold great promise and should be investigated further. In particular, a piezoelectric pump was shown to have the potential for power densities approaching those of hydraulics, as well as high steady-state efficiency, and no wasted power holding a load. The magnetostrictive actuators are similar to piezoelectric actuators, but with lower anticipated efficiencies and load holding capability similar to that of electric motors. Power electronics is an issue for both that is the subject of ongoing research. 
Table 24. Comparison of conventional actuators

\begin{tabular}{|c|c|c|c|}
\hline Item & Electromagnetic Actuators & Hydraulic Actuators & Pneumatic Actuators \\
\hline Bandwidth & $\begin{array}{l}\text { Transmission required. Motors } \\
\text { sized } 10 \text { to } 20 \% \text { larger due to } \\
\text { transmission efficiencies. }\end{array}$ & $\begin{array}{l}\text { High performance servos in the } \\
50 \text { to } 100 \mathrm{~Hz} \text { range. Dependent } \\
\text { on actuator and controller } \\
\text { design. }\end{array}$ & 5 to $6 \mathrm{x}$ less than hydraulics. \\
\hline $\begin{array}{l}\text { Power to } \\
\text { Weight }\end{array}$ & $\begin{array}{l}\text { Approximately } 1 / 10 \text { hydraulics } \\
\text { ratio. }\end{array}$ & Excellent. & $\begin{array}{l}\text { Elastic type actuators are } \\
\text { similar to hydraulics. Hard- } \\
\text { shell type actuators are similar } \\
\text { to electric actuators. } \\
\end{array}$ \\
\hline $\begin{array}{l}\text { Power to } \\
\text { Volume }\end{array}$ & $\begin{array}{l}\text { Approximately } 1 / 5 \text { hydraulics } \\
\text { ratio. }\end{array}$ & Excellent. & \begin{tabular}{|l|} 
Elastic type actuators are \\
similar to hydraulics. Hard- \\
shell type actuators are similar \\
to electric actuators. \\
\end{tabular} \\
\hline Packaging & $\begin{array}{l}\text { Transmission increases vol by } \\
20-50 \% \text {. Power electronics can } \\
\text { exceed size of actuator. }\end{array}$ & $\begin{array}{l}\text { No transmission. Power } \\
\text { electronics are inconsequential. } \\
\text { Sound proofing pumps } \\
\text { difficult. }\end{array}$ & Similar to hydraulics. \\
\hline $\begin{array}{l}\text { Load } \\
\text { Holding }\end{array}$ & $5-10 \%$ or rated power required. & $\begin{array}{l}\text { No power for linear actuators } \\
\text { with asymetrical cylinders. } 1 \text { to } \\
10 \% \text { for symetrical cylinders } \\
\text { and rotary actuators. }\end{array}$ & Similar to hydraulics. \\
\hline
\end{tabular}




\section{REFERENCES}

1. AeroVironment, Inc., "Final Report for Exoskeletons for Human Performance Augmentation Power System Development," AV-50619/03, AeroVironment, Inc., Monrovia, Calif., 2003.

2. Amendola, et al., "An Ultrasafe Hydrogen Generator: Aqueous, Alkaline Borohydride Solutions and Ru Catalyst," Journal of Power Sources 85, 186-189 (2000).

3. Andersen, B. W., The Analysis and Design of Pneumatic Systems, Krieger Publishing Co., Malabar, Florida. 1967.

4. Andriulli, J. B., et al., Advanced Power Generation Systems for the $21^{\text {st }}$ Century: Market Survey and Recommendations for a Design Philosophy, ORNL/TM-1999/213, Oak Ridge National Laboratory, Oak Ridge, Tenn., November 1999.

5. Antonsson E. K. and R. W. Mann, "The Frequency Content of Gait," J Biomech 18(1), 39-47 (1985).

6. Barter J, I. Emanuel, B. Truett, A Statistical Evaluation of Joint Range Data, WADC Technical Note 57-311, Wright Air Development Center, 1957.

7. Beatty, H. A., U.S. Dept of Commerce, PB-6330, 1944.

8. Boynton, A. C., and H. P. Crowell, A Human Factors Investigation of Exoskeleton Boot Interface Sole Thickness, U.S. Army Research Laboratory, Aberdeen Proving Ground, Md. (technical report in press).

9. Brown, H. C. and C. A. Brown, Journal of the American Chemical Society 84, 1493 (1962).

10. Bunch, W. H., Atlas of Orthotics/American Academy of Orthopaedic Surgeons, Mosby, 1985.

11. Crowell, H. P., A. C. Boynton, and M. Mungiole, Exoskeleton Power and Torque Requirements Based on Human Biomechanics, ARL-TR-2764, U.S. Army Research Laboratory, Aberdeen Proving Ground, Md., November 2002.

12. Crowell, H. P., Human Engineering Design Guidelines for a Powered, Full Body Exoskeleton, ARL-TN-60, U.S. Army Research Laboratory, Aberdeen Proving Ground, Md., July 1995.

13. Dowling, K., Power Sources for Small Robots, CMU-RI-TR-97-02, Carnegie Mellon University. January 1997.

14. Chou, C. P. and Hannaford, B., Measurements and Modeling of McKibben Pneumatic Artificial Muscles. IEEE Transactions on Robotics and Automation, 12(1), 90-102 (1996).

15. Garret, D. E. Borates, Handbook of Deposits, Processing, Properties, and Use Academic Press, San Diego, 1998.

16. Goldfarb, M. and W. Durfee, "Design of a Controlled-Brake Orthosis for FES-Aided Gait," IEEE Trans. Rehab. Eng. 4(1), 13-24 (1996). 
17. Harris, G. F., K. R. Acharya, and R. A. Bachschmidt, "Investigation of Spectral Content from Discrete Plantar Areas During Adult Gait: An Expansion of Rehabilitation Technology," IEEE Trans Rehab. Eng. 4(4), 360-74 (1996).

18. Heywood, J. B., Internal Combustion Engine Fundamentals, McGraw-Hill, New York, 1998.

19. Hirschenhofer, J. H., D. B. Stauffer, R. R. Engleman, and M. R. Klett, Fuel Cell Handbook, Business/Technology Books for the U.S. Department of Energy, 1994.

20. Hollerbach, J. M., I. W. Hunter, and J. Ballantyne, "A Comparative Analysis of Actuator Technologies for Robotics," pp. 299-342 in The Robotics Review 2, ed. O. Khatib, J. Craig, T. Perez, The MIT Press, Cambridge, Mass., 1992.

21. Jansen, J. F. et al., Exoskeleton for Soldier Enhancement Systems Feasibility Study, ORNL/TM-2000/256, Oak Ridge National Laboratory, Oak Ridge, Tenn., September 2000.

21. Kaufman, C. M. and B. Sen, Journal of the Chemical Society Dalton Transactions, 307 (1985).

22. Kellogg, R. and A. Flatau, "Blocked Force Investigation of a Terfenol-D Transducer," Proc. SPIE Conf. on Smart Structures and Integrated Systems, Newport Beach, Calif., March 1999, pp. 184-195.

23. Legg, S. J., T. Ramsey, D. J. Knowles, "The Metabolic Cost of Backpack and Shoulder Load Carriage," Ergonomics 35(9), 1063-1068 (1992).

24. Levy, A., J. B. Brown, and C. J. Lyons, Ind. Eng. Chem. 52, 211 (1960).

25. McMahon, T. A., "Mechanics of Locomotion," The International Journal of Robotics Research 3(2), 4-28 (1984).

26. Merritt, H. E., Hydraulic Control Systems, John Wiley and Sons, New York, 1989.

27. Miller, T. J., Brushless Permanent Magnet and Reluctance Motor Drives, Oxford University Press, Oxford, U.K., 1989.

28. Nasser, K. D., J. Leo, and H. H. Cudney, "Compact Piezohydraulic Actuation System," SPIE Paper Number 3991-41: Annual Symposium on Smart Structures and Materials, 2000 .

29. Nawoczenski, D. A. and M. E. Epler, Orthotics in Functional Rehabilitation of the Lower Limb, Saunders, 1997.

30. Newton, D. V., J. A. Main, E. Garcia, and L. Massengill, "Piezoelectric Actuation Systems: Optimization of Driving Electronics," pp. 259-266 in Proc. of SPIE's 1996 North American Symposium on Smart Structures and materials: Smart Structures and Integrated Systems, Vol. 2717, San Diego, Calif., February 1996.

31. Park, S. E. and T. R. Shrout, "Characteristics of Relaxor-Based Piezoelectric Single Crystals for Ultrasonic Transducers," IEEE Transactions on Ultrasonics, Ferroelectrics, and Frequency Control 44(5), 1140-1146, (1997).

32. Redford, J. B., Orthotics Etcetera, Williams \& Wilkins, 1986.

33. Rose J. and J. Gamble, Human Walking, 2nd ed., Williams \& Wilkins, 2000. 
34. Sanders J. E., B. S. Goldstein, and D. F. Leotta, "Skin Response to Mechanical Stress: Adaptation Rather than Breakdown - A Review of the Literature," J Rehab Res. Dev. 32(3), 214-228 (1995).

35. Sanders J.E,. J. M. Greve, S. B. Mitchell, and S. G. Zachariah, "Material Properties of Commonly-Used Interface Materials and Their Static Coefficients of Friction with Skin and Socks," J. Rehabil. Res. Dev. 35(2), 161-76 (1998).

36. Schlesinger, H. I., "Final Report to Signal Corps Ground Signal Agency on Contract W3434-SC-174", PB-6331, 1944.

37. Schlesinger, H. I., et al., Journal of the American Chemical Society 75, 215-219 (1953).

38. Sodium Borohydride Digest, Morton International, Inc., 1995.

39. Theis, T. J., J. C. Conklin, J. F. Thomas, and T. R. Armstrong, Comparison of Prime Movers Suitable for USMC Expeditionary Power Sources, ORNL/TM-2000/116, Oak Ridge National Laboratory, Oak Ridge, Tenn., March 2000.

40. Thomas, S. and M. Zalbowitz, Fuel Cells - Green Power, LA-UR-99-3231, Los Alamos National Laboratory, Los Alamos, N. Mex., 1999.

41. Tylee, L., "On-Line Failure Detection in Nuclear Power Plant Instrumentation," IEEE Trans. Automat. Contr., AC-28, 406-415, March 1983.

42. Wakizoe, M., O. Velev, and S. Srinivasan, Electrochemica Acta 40(3), 335-344 (1995).

43. Wax, S. G. and R. R. Sands, "Electroactive Polymer Actuators and Devices," SPIE Conference of Electroactive Polymer Actuators and Devices, Newport Beach, Calif., March 1999, pp. 2-9.

44. Winter, D. F., Biomechanics and Motor Control of Human Movement, Wiley, 1990.

45. Woodson, W. E., B. Tillman, and P. Tillman, Human Factors Design Handbook, 2nd ed, Mc-Graw Hill, 1992. 
ORNL/TM-2003/216

\section{INTERNAL DISTRIBUTION}

1. J. F. Birdwell

2. W. E. Dixon

3. E. C. Fox

4. R. G. Gilliland

5. D. J. Hill

6. J. F. Jansen

7. S. M. Killough

8. R. F. Lind

9. P. D. Lloyd
10. L. J. Love

11. M. W. Noakes

12. F. G. Pin

13. B. S. Richardson

14. J. C. Rowe

15. R. B. Shelton

16. ORNL Central Research Library

17. ORNL Laboratory Records - RC

18. ORNL Laboratory Records - OSTI

\section{EXTERNAL DISTRIBUTION}

(This document was cleared by DARPA and has been approved for Public Release, Distribution Unlimited.)

19. Army Research Laboratory, AMSRL-HR-SB, Attn: Angela Boynton (Bldg. 459), Aberdeen Proving Ground, MD 21005-5425

20. Army Research Laboratory, AMSRL-HR-SB, Attn: Philip Crowell (Bldg. 459), Aberdeen Proving Ground, MD 21005-5425

21. W. K. Durfee, University of Minnesota - Twin Cities, Mechanical Engineering, Room 210B, 111 Church St., SE, Minneapolis, MN 55455

22. Greg Fischer, Engineering Consultant, Strategic Analysis, Inc., 3601 Wilson Blvd., Suite 500, Arlington, VA 22201

23. John Gongola, AeroVironment, Inc., 825 S. Myrtle Ave., Monrovia, CA 91016

24. D. J. Leo, Virginia Polytechnic Institute and State University, Center for Intelligent Material Systems and Structures, Mechanical Engineering Department, Blacksburg, VA 24061-0261

25. John A. Main, DARPA-DSO, 3701 N. Fairfax Dr., Arlington, VA 22202-1714

26. George Malosh, ORNL Site Manager, U.S. Department of Energy, Oak Ridge National Laboratory, Post Office Box 2008, Oak Ridge, TN 37831-6269

27. Paul McCready, AeroVironment, Inc., 825 S. Myrtle Ave., Monrovia, CA 91016

28. Army Research Laboratory, AMSRL-CI-EE, Attn: Michael Mungiole, 2800 Powder Mill Road, Adelphi, MD 20783-1197

29. O. A. Velev, AeroVironment, Inc., 825 S. Myrtle Ave., Monrovia, CA 91016

30. T. Zambrano, AeroVironment, Inc., 825 S. Myrtle Ave., Monrovia, CA 91016 